\title{
Depression and apathy after stroke
}

Citation for published version (APA):

Douven, E. (2017). Depression and apathy after stroke: The influence of risk factors on their development and course. [Doctoral Thesis, Maastricht University]. NeuroPsych Publishers.

https://doi.org/10.26481/dis.20171208ed

Document status and date:

Published: 01/01/2017

DOI:

10.26481/dis.20171208ed

Document Version:

Publisher's PDF, also known as Version of record

\section{Please check the document version of this publication:}

- A submitted manuscript is the version of the article upon submission and before peer-review. There can be important differences between the submitted version and the official published version of record.

People interested in the research are advised to contact the author for the final version of the publication, or visit the DOI to the publisher's website.

- The final author version and the galley proof are versions of the publication after peer review.

- The final published version features the final layout of the paper including the volume, issue and page numbers.

Link to publication

\footnotetext{
General rights rights.

- You may freely distribute the URL identifying the publication in the public portal. please follow below link for the End User Agreement:

www.umlib.nl/taverne-license

Take down policy

If you believe that this document breaches copyright please contact us at:

repository@maastrichtuniversity.nl

providing details and we will investigate your claim.
}

Copyright and moral rights for the publications made accessible in the public portal are retained by the authors and/or other copyright owners and it is a condition of accessing publications that users recognise and abide by the legal requirements associated with these

- Users may download and print one copy of any publication from the public portal for the purpose of private study or research.

- You may not further distribute the material or use it for any profit-making activity or commercial gain

If the publication is distributed under the terms of Article $25 \mathrm{fa}$ of the Dutch Copyright Act, indicated by the "Taverne" license above, 


\section{DEPRESSION AND APATHY AFTER STROKE}

The influence of risk factors on their development and course

\section{Elles Douven}


Cover design: Iliana Boshoven | AgileColor.com

Layout: Iliana Boshoven | AgileColor.com

Printed by: Ridderprint BV, Ridderkerk

Published by: NeuroPsych Publishers

ISBN:

978-94-6299-769-1

Copyright (c) 2017 Elles Douven, Maastricht

All rights reserved. No part of this publication may be reproduced or transmitted, in any form or by any means, without the prior written permission of the author. 


\section{DEPRESSION AND APATHY AFTER STROKE}

The influence of risk factors on their development and course

PROEFSCHRIFT

Ter verkrijging van de graad van doctor aan de Universiteit Maastricht, op gezag van de Rector Magnificus, Prof. dr. Rianne M. Letschert, volgens het besluit van het College van Decanen, in het openbaar te verdedigen op vrijdag 8 december 2017 om 10:00 uur

door

\section{Elles Douven}

Geboren op 19 februari 1991 te Nederweert-Eind 


\section{PROMOTOR}

Prof. dr. F.R.J. Verhey

\section{CO-PROMOTORES}

Dr. P. Aalten

Dr. J. Staals

\section{BEOORDELINGSCOMMISSIE}

Prof. dr. P.A.M. Hofman (voorzitter)

Dr. A.A. Duits

Dr. A.F.G. Leentjens

Prof. dr. F.E. de Leeuw (Radboud UMC, Nijmegen)

Prof. dr. J.M.A. Visser-Meily (UMC Utrecht)

The research described in this thesis was performed at the Department of Psychiatry and Neuropsychology, School for Mental Health and Neuroscience, Maastricht University, Alzheimer Center Limburg, Maastricht, the Netherlands.

The studies presented in this thesis were financially supported by Maastricht University, Health Foundation Limburg, and the Adriana van Rinsum-Ponsen Stichting.

Financial support by the Dutch Heart Foundation for the publication of this thesis is gratefully acknowledged. 


\section{CONTENTS}

CHAPTER 1 General introduction, thesis aim and outline

Neuropraxis, 2014

CHAPTER 2 Imaging markers of post-stroke depression and apathy:

a systematic review and meta-analysis

Neuropsychology Review, 2017

CHAPTER 3 The Cognition and Affect after Stroke: a Prospective

Evaluation of Risks (CASPER) study: rationale and design

BMC Neurology, 2016

CHAPTER 4 Temporal associations between fatigue, depression, and apathy after stroke: results of the Cogniton and Affect after Stroke, a Prospective Evaluation of Risks study

Cerebrovascular Diseases, 2017

CHAPTER 5 Premorbid personality and course of symptoms of depression and apathy after stroke: results of the CASPER study Submitted

CHAPTER 6 Imaging markers associated with the development of poststroke depression and apathy: results of the CASPER study Submitted

CHAPTER 7 Baseline vascular cognitive impairment predicts the course of apathetic symptoms after stroke: the CASPER study American Journal of Geriatric Psychiatry, 2017

CHAPTER 8 Depression-Executive Dysfunction Syndrome after stroke: results of the CASPER study

Submitted

CHAPTER 9 General discussion

Nederlandse samenvatting (Dutch summary)

Knowledge valorization

Dankwoord (Acknowledgements)

Thesis defenses from $\mathrm{MHeNs}$

List of publications

Curriculum Vitae 
CHAPTER 1 


\section{GENERAL INTRODUCTION THESIS AIM AND OUTLINE}

Partly published in:

Neuropsychiatrische stoornissen na een beroerte. Neuropraxis 2014; 18(5), 151-161 Syenna HJ Schievink, Elles Douven, Pauline Aalten, Sebastian Köhler 
Chapter 1 


\section{GENERAL INTRODUCTION}

Stroke is one of the leading causes of disability in the world. ${ }^{1}$ Worldwide, stroke incidence is estimated around 17 million events each year, ${ }^{2}$ and approximately 50 million stroke survivors currently live with remaining deficits that limit their daily functioning. ${ }^{3}$ In the Netherlands, stroke affects around 41,000 individuals each year, ${ }^{4}$ and this number increases steadily due to the aging population. ${ }^{5}$ Though the length of hospitalization after stroke decreased in the past years due to better acute treatment, ${ }^{6}$ stroke is responsible for $2.5 \%$ of the total health care costs. ${ }^{7}$ Currently, more than 240,000 individuals deal with the consequences of stroke in daily life. ${ }^{8}$ Beside motor, sensory, and speech deficits, cognitive deficits and neuropsychiatric symptoms like depression and apathy are frequent after stroke, occurring in around $30 \%$ of stroke survivors. ${ }^{9-11}$

Neuropsychiatric symptoms are associated with poor prognosis, as these have a negative influence on functional recovery ${ }^{12-14}$ and quality of life after stroke..$^{15,16}$ Despite their high frequency, neuropsychiatric symptoms are less salient compared with physical, visual, speech, or cognitive dysfunctions. They are therefore frequently under-recognized in clinical practice,${ }^{17}$ and treatment needs of these stroke patients are often unmet. ${ }^{18}$ Post-stroke depression (PSD) and post-stroke apathy (PSA) are two of the most common neuropsychiatric outcomes after stroke, and have substantial overlap in symptoms. ${ }^{19}$ Particularly, the phenomenological overlap in the key criterion 'loss of interest' makes it difficult to differentiate between these two syndromes. ${ }^{20}$ Several studies found indications for differences between PSD and PSA, though there remains discussion whether these support the hypothesis that PSD and PSA are two independent syndromes.

\section{PREVALENCE AND DIAGNOSIS OF PSD}

According to a meta-analysis, PSD occurs in approximately $31 \%$ of the stroke population at any time point after stroke. ${ }^{9}$ PSD is associated with an increased risk for cognitive impairment ${ }^{21}$ and has a large impact on long-term prognosis, as it results in increased mortality ${ }^{22,23}$ and more disability. ${ }^{20}$ According to the fifth edition of the Diagnostic and Statistical Manual of Mental Disorders (DSM-5), PSD is categorized as a mood disorder due to another medical condition, and the diagnostic criteria are described in Table 1. ${ }^{24} \mathrm{PSD}$ can develop in the acute period after stroke, but also later in the course, i.e. in the sub-acute or chronic stage.

A major difficulty in diagnosing depression in stroke survivors is to make sure that the somatic depression symptoms are related to mood and are not the result of the stroke itself, as symptoms like psychomotor retardation, changes in appetite, or fatigue are frequent after stroke. ${ }^{25}$ However, Cumming et al. ${ }^{26}$ showed that patients 
with PSD show roughly the same profile of depressive symptoms compared with depressed individuals in the general population, although patients with PSD were less likely to report anhedonia. Despite their medical condition, patients with PSD were thus no more likely than depressed individuals in the general population to report somatic symptoms over psychological symptoms. ${ }^{26}$ Hence, it has been suggested that clinicians should not exclude the somatic symptoms in diagnosing PSD, but these need to be considered separately, next to psychological and neurocognitive symptoms of depression. ${ }^{25,26}$

Table 1. Diagnostic criteria for depression due to stroke according to the fifth edition of the Diagnostic and Statistical Manual of Mental Disorders ${ }^{24}$

\section{For a diagnosis of depression due to stroke the patient should fulfill the criteria $A, B, C$ and $D$}

A. A prominent and persistent period of lowered mood (2 weeks or longer) or clear loss of interest or pleasure in all, or almost all, activities.

B. At least four of the following symptoms:

Substantial weight loss or gain

Insomnia or hypersomnia

Psychomotor agitation or retardation

Fatigue or loss of energy

Worthlessness or inappropriate guilt

Diminished concentration, or indecisiveness

C. There should be evidence that the depressive disorder is due to a somatic condition (i.e. stroke), and cannot be better explained by another psychic disorder.

D. The depressive disorder is not the result of a delirium, causes clinically significant distress, and results in impairments in social, professional, or other important areas of functioning.

\section{ETIOLOGY AND PREDICTORS OF PSD}

Previous research on PSD focused mainly on its etiological factors. Several studies examined whether certain stroke lesion locations could be identified that are associated with a higher prevalence of PSD. Robinson and colleagues were the first to find such an association in 1981, by suggesting that patients with left hemispheric lesions presented with PSD more frequently than patients with right hemispheric lesions, and the severity of depressive symptoms directly correlated with the closeness of the lesion to the anterior pole. ${ }^{27}$ This finding has led to the 'lesion location hypothesis' of PSD, stating that PSD is associated with left frontal lesions. However, this hypothesis has remained controversial ever since, and meta-analyses could not support this relationship. ${ }^{28-30}$ In addition to the left frontal lobe, associations have been found with (left) basal ganglia lesions. ${ }^{31-33}$ Also, lesions in fronto-subcortical regions were found to be involved in PSD, ${ }^{34,35}$ which seems biologically plausible, as these brain areas are known to be involved in regulation of mood. ${ }^{36}$ According to a study 
in 275 ischemic stroke survivors, PSD is associated with lesions in fronto-subcortical circuits $^{37}$ and is often presented together with vascular cognitive impairment (VCl). $\mathrm{VCl}$ occurs frequently after stroke and is defined as the establishment of a cognitive disorder with vascular disease being the dominant pathology underlying the cognitive impairment. ${ }^{38}$ Executive dysfunction is particularly frequent after stroke and co-occurs with PSD. ${ }^{39}$ This so-called 'Depression-Executive Dysfunction Syndrome (DES)' might represent a PSD subtype, and has been suggested to be associated with worse stroke outcome. In a longitudinal study in 116 first-ever stroke patients, ${ }^{40}$ patients with DES showed less recovery from executive dysfunction (ED) and depression compared with patients with ED-only or PSD-only, respectively. Also, in a 12-year follow-up study, PSD was associated with more rapid stroke recurrence ${ }^{41}$ and increased mortality risk, ${ }^{42}$ and this was further increased in patients with DES.

Beside stroke lesion location, also large lesion volume 43,44 and lesion severity ${ }^{45}$ seem to be associated with PSD. In contrast with lesion-related markers, generalized markers of vascular brain pathology like white matter hyperintensities (WMH), cerebral microbleeds, (silent) old infarcts, as well as degenerative pathology like cerebral atrophy are less frequently studied in association with PSD, though there are suggestions that they play a role in the development of PSD as well. ${ }^{46-49}$ Furthermore, there are hypotheses that PSD may be related to an increased inflammatory response ${ }^{50}$ and also genetic polymorphisms in the serotonin system might be involved. ${ }^{51}$

In addition to abovementioned biological factors, psychosocial mechanisms are suggested to be involved in the development of PSD. ${ }^{52}$ Personality traits, mainly neuroticism, ${ }^{53}$ a passive coping style, ${ }^{52}$ and poor social support ${ }^{54}$ are psychosocial factors shown to be associated with PSD. A meta-analysis of 19 cross-sectional studies showed that post-stroke fatigue (PSF) is associated with PSD. ${ }^{55}$ However, until now it remains unclear whether PSD results in PSF, whether PSF results in PSD, or whether the relationship between PSD and PSF is bi-directional. ${ }^{56}$ For this, longitudinal studies are needed that study this temporal relationship in both directions. ${ }^{57,58}$ Another possibility may be that both are caused by a third unknown factor.

Taken together, the pathogenesis of PSD is probably a combination of both biological and psychosocial mechanisms, ${ }^{54,59-61}$ which are summarized in Figure 1. It is likely that psychosocial factors interact with biological factors, and the latter is probably more than lesion location and also includes background vascular and degenerative brain pathology. Thus, both psychosocial and biological factors are contributing to the development of PSD, with each factor having its major impact at a different time point post-stroke. ${ }^{61}$ PSD itself may also have an effect on some of these biological and psychosocial factors in a bi-directional fashion, like the functioning of frontalsubcortical circuits, cognition, and fatigue, resulting in a complex interplay between these processes. 


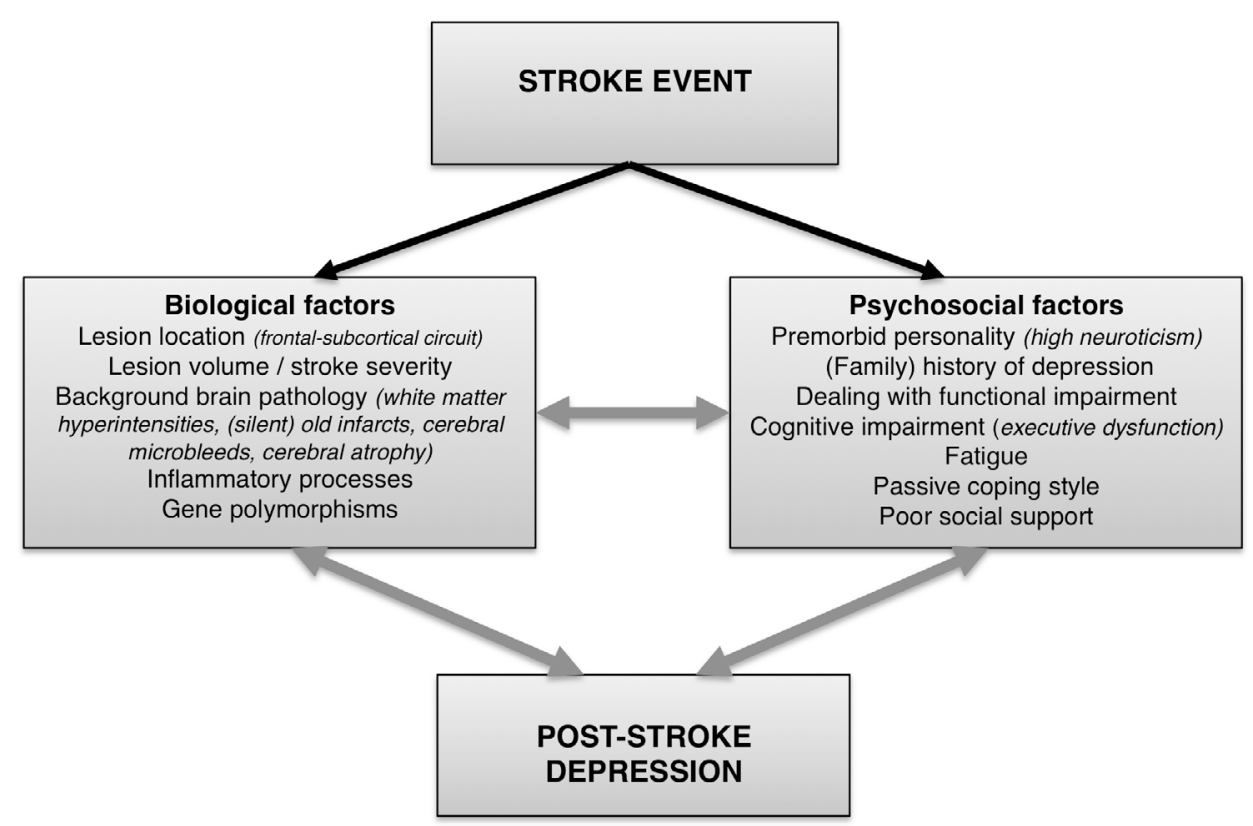

Figure 1. Biological and psychosocial processes involved in the etiology of post-stroke depression

\section{PREVALENCE AND DIAGNOSIS OF PSA}

PSA is frequently reported after stroke, with an estimated pooled prevalence of almost 35\%,62 which is approximately equally frequent as PSD. 11,62 Nevertheless, PSA is often not recognized as a syndrome on its own, both in research and in clinical practice. PSA has shown to be negatively associated with rehabilitation outcome $^{43}$ and presents more often in the post-acute and chronic stroke phase. Levels of apathy tend to remain relatively stable during the first year post-stroke. ${ }^{63,64}$

The DSM-5 does not define diagnostic criteria for apathy. According to Marin, ${ }^{65}$ who was the first to define apathy as a distinct syndrome, apathy can be defined as a loss of motivation or lack of interest, characterized by reduced goal-directed behavior and cognitive activity, and emotional indifference. These criteria were adapted by including the criterion that apathy should not be comorbid with depression or cognitive impairment, ${ }^{66}$ and apathetic symptoms should be present during the majority of the day for at least four consecutive weeks. ${ }^{20}$ During a consensus meeting in 2008 , these criteria were revised and formulated in such a way that they can be applied to the occurrence of apathy in various neuropsychiatric disorders or conditions (Table 2). ${ }^{67}$ 
Table 2. Diagnostic criteria for apathy from Robert et al. (2009)

\begin{tabular}{l} 
For a diagnosis of apathy the patient should fulfill the criteria $A, B, C$ and $D$ \\
\hline A. Loss of or diminished motivation in comparison to the patient's previous level of functioning \\
and which is not consistent with his age or culture. These changes in motivation may be re- \\
ported by the patient himself or by the observations of others.
\end{tabular}

Domain B1 (Behavior): Loss of, or diminished, goal-directed behavior as evidenced by at least one of the following:

- Loss of self-initiated behavior (for example: starting conversation, doing basic tasks of dayto-day living, seeking social activities, communicating choices).

- Loss of environment-stimulated behavior (for example: responding to conversation, participating in social activities).

Domain B2 (Cognition): Loss of, or diminished, goal-directed cognitive activity as evidenced by at least one of the following:

- Loss of spontaneous ideas and curiosity for routine and new events (i.e., challenging tasks, recent news, social opportunities, personal/family and social affairs).

- Loss of environment-stimulated ideas and curiosity for routine and new events (i.e., in the persons residence, neighborhood or community).

Domain B3 (Emotion): Loss of, or diminished, emotion as evidenced by at least one of the following:

- Loss of spontaneous emotion, observed or self-reported (for example, subjective feeling of weak or absent emotions, or observation by others of a blunted affect).

- Loss of emotional responsiveness to positive or negative stimuli or events (for example, observer-reports of unchanging affect, or of little emotional reaction to exciting events, personal loss, serious illness, emotional-laden news).

C. The symptoms (A-B) cause clinically significant impairment in personal, social, occupational, or other important areas of functioning.

The symptoms (A-B) are not exclusively explained or due to physical disabilities (e.g. blindness

D. and loss of hearing), to motor disabilities, to diminished level of consciousness, or to the direct physiological effects of a substance (e.g. drug of abuse, a medication).

\section{ETIOLOGY AND PREDICTORS OF PSA}

Compared with PSD, the etiology of PSA has received far less attention. With regards to biological factors, PSA has been associated with damage to subcortical areas, mainly the basal ganglia. ${ }^{11,68} \mathrm{Also}, \mathrm{WMHs}$ in the right fronto-subcortical circuit have been associated with PSA. ${ }^{69}$ According to a meta-analysis by Caeiro et al. ${ }^{70}$ the frequency of PSA was not associated with stroke lesion laterality or lesion type. However, PSA was significantly associated with older age, recurrent stroke, cognitive impairment, and depression. Also, patients with acute-phase PSA showed an almost four-fold risk of having PSA at one-year follow-up, ${ }^{70}$ suggesting 
that PSA can be persistent over time. The meta-analysis by van Dalen et al. ${ }^{11}$ showed that PSA is associated with cognitive impairment and increased disability. Due to the lack of longitudinal studies examining risk factors of PSA, it remains unclear how these factors influence the development of PSA over time.

\section{THE RELATIONSHIP BETWEEN PSD AND PSA}

Around 40 percent of patients with PSA show comorbid PSD, while in the remaining 60 percent PSA occurs as an independent syndrome. ${ }^{11}$ According to the meta-analysis by Caeiro et al., ${ }^{70}$ PSA without PSD occurs twice as often than PSD without PSA, which supports the idea that PSA frequently occurs as an independent clinical entity. A previous study that attempted to disentangle the independence of PSD and PSA found ambiguous results. While at three to six months post-stroke the two syndromes tended to occur independently, ${ }^{69}$ at 12-month follow-up the co-occurrence of PSA and PSD increased significantly. ${ }^{63}$ This suggests that the relationship between PSD and PSA is probably dependent on the time of measurement.

Although lack of motivation or interest plays a role in both apathy and depression, apathy is considered to be a motivational disorder, whereas depression is mainly seen as a disorder of lowered mood. ${ }^{71}$ Next to an overlap in clinical symptomatology, there is also evidence for an overlap in associated risk factors. Both PSD and PSA are related to lesions in fronto-subcortical circuits ${ }^{11,37}$ and with increased WMH severity. ${ }^{48,69}$ In addition, cognitive impairment, ${ }^{11,21}$ and impaired functional outcome, ${ }^{13,21}$ are also associated with the development of both. However, there are also suggestions for differences between these two syndromes. From a biological perspective, different neurotransmitter systems may be involved in the development of depression and apathy. For instance, the dopaminergic system has been found to play an important role in apathy, whereas the serotonergic system seems to play an important role in the development of depression. ${ }^{72,73}$ In addition, different stroke lesion locations have been shown to be associated with PSD and PSA, ${ }^{35}$ and diffusion tensor imaging studies have found that white matter tracks subserving different networks are damaged in PSD ${ }^{74}$ and PSA. ${ }^{75}$ This suggests that disconnection of different brain networks are involved in these syndromes. As there might be a differential role for neurotransmitter systems underlying these syndromes, this may also have consequences for treatment recommendations. A meta-analysis showed that PSD seems to respond well to antidepressants, which are usually working on the serotonergic system. ${ }^{76}$ PSA might benefit from different treatment like dopaminergic agents or cholinesterase inhibitors, ${ }^{77}$ though this has not been confirmed in randomized-controlled trials. Importantly, the use of selective serotonin reuptake inhibitors, which are commonly prescribed 
antidepressants, may worsen symptoms of apathy, ${ }^{78}$ which is an additional reason why screening for apathy and recognizing its independence from depression is necessary.

\section{GAPS IN CURRENT KNOWLEDGE}

Several studies found indications for differences between PSD and PSA, though associations between PSA and specific risk factors have been less frequently studied compared with PSD. Beside suggestions for differences, there is also evidence for a significant overlap in associated factors of PSD and PSA (e.g. cognitive impairment, poor functional outcome, lesions in frontal-subcortical areas). However, these findings were largely based on cross-sectional data. It is therefore poorly understood if and how both biological and psychosocial factors have a differential influence on the development and course of PSD and PSA over time. A better understanding of the role of these factors may result in better recognition, distinction, and earlier detection of these conditions. This insight may also be valuable for providing patient-tailored treatment options for patients with PSD and PSA, leading to a better clinical prognosis after stroke.

\section{THESIS AIM AND OUTLINE}

The main aim of this thesis is to examine the association of biological and psychosocial risk factors on the development and course of PSD and PSA, and whether differences can be found between the associations with these two syndromes. The Cognition and Affect after Stroke: a Prospective Evaluation of Risks (CASPER) study is a one-year prospective clinical cohort follow-up study designed to examine whether stroke-specific, cerebrovascular and neurodegenerative markers, and psychosocial factors are associated with cognitive impairment, PSD, and PSA, and whether they predict their course over 12 months. The rationale and design of the CASPER study is described in Chapter 3 and data from the CASPER study were used in Chapters 4 to 8 . The research described in the chapters is based on the following research questions:

\section{1) Which imaging markers have been associated with PSD and PSA in previous studies?}

Chapter 2 provides a systematic literature review, which gives a comprehensive and up-to-date evaluation of imaging markers that have been associated with PSD and PSA. In addition, meta-analyses were performed to examine the association between PSD and PSA and stroke lesion laterality, type, and location. Differences and similarities in the brain correlates associated with PSD and PSA were examined. 
2) Is there evidence for a bi-directional relationship between PSF and PSD, and PSF and PSA?

In Chapter 4, the temporal relationships between PSF and PSD, and between PSF and PSA are disentangled. The effect of baseline PSF on the course of depressive and apathetic symptoms was examined, and also the effect of baseline PSD and PSA on the course of fatigue symptoms. In addition, we tested whether change in fatigue levels from baseline to follow-up was associated with change in levels of depression and apathy during the same period.

3) Are premorbid personality traits predictive for the course of PSD and PSA?

Chapter 5 examines whether premorbid personality traits are a predictive factor for the development and course of PSD and PSA by studying the influence of neuroticism, extraversion, openness, conscientiousness, and agreeableness on depressive and apathetic symptoms over the course of 12 months.

4) Which lesion-related and generalized vascular and degenerative imaging markers are predictive for the development of PSD and PSA?

Chapter 6 studies the association between both lesion-related imaging markers and more generalized imaging markers like $\mathrm{WMH}$, presence of old infarcts and microbleeds, cerebral small vessel disease burden, and atrophy assessed at three months post-stroke and the development of PSD and PSA over a period of 12 months follow-up.

5) What is the influence of vascular cognitive impairment on the course of PSD and PSA?

In Chapter 7, the association between presence of cognitive impairment in any cognitive domain (memory, information processing speed, or executive function), and degree of $\mathrm{VCl}$ (i.e. impairment in single or multiple cognitive domains) and the development of PSD and PSA is examined, and also how domain-specific VCl influences the course of PSD and PSA over a period of 12 months.

6) Do executive dysfunctions denote a distinct PSD subtype based on clinical correlates and prognosis?

Chapter 8 studies whether patients with DES, a potential biological subtype of depression in which depression is paired with executive dysfunction, show differences in demographic and clinical characteristics, and stroke-related or generalized vascular and degenerative brain pathology compared with patients with only depression, or only executive dysfunction. In addition, differences in the course of executive dysfunction, depressive symptoms, and other cognitive domains between patients with and without DES are studied. 
Chapter 9 provides a general discussion, including a summary of the main findings of the various chapters, and implications and recommendations for future research and clinical practice. 


\section{REFERENCES}

1. Murray CJ, Vos T, Lozano R, Naghavi M, Flaxman AD, Michaud C, et al. Disability-adjusted life years (dalys) for 291 diseases and injuries in 21 regions, 1990-2010: A systematic analysis for the global burden of disease study 2010. Lancet. 2013;380:2197-2223.

2. Feigin VL, Forouzanfar MH, Krishnamurthi R, Mensah GA, Connor M, Bennett DA, et al. Global and regional burden of stroke during 1990-2010: Findings from the global burden of disease study 2010. Lancet. 2014;383:245-255.

3. Bonita R, Mendis S, Truelsen T, Bogousslavsky J, Toole J, Yatsu F. The global stroke initiative. Lancet Neurol. 2004;3:391-393.

4. Dutch Hearth Foundation. Facts and data about a stroke [webpage on the internet]. [Cited 2017 May 30]; updated 2015. Available from: https://http://www.hartstichting.nl/hart-vaten/cijfers/ beroerte.

5. Vat L, Middelkoop I, Buijck B, Minkman M. The development of integrated stoke care in the netherlands a benchmark study. Int J Integr Care. 2016;16:1-10.

6. National Institute for Health and Environment. Hospitalization and treatment of stroke [webpage on the internet]. [Cited 2017 May 30]; Available from: https://http://www.volksgezondheidenzorg. info/onderwerp/beroerte/preventie-zorg/zorg.

7. National Institute for Health and Environment. Costs of stroke care management [webpage on the internet]. [Cited 2017 May 30]; updated 2011. Available from: https://http://www. volksgezondheidenzorg.info/onderwerp/beroerte-kosten/kosten.

8. Vaartjes I, Koopman C, van Dis I, Visseren F, Bots ML. Hart- en vaatziekten in Nederland 2013, cijfers over leefstijl, risicofactore, ziekte en sterfte. Den Haag: Hartstichting.

9. Hackett ML, Pickles K. Part i: Frequency of depression after stroke: An updated systematic review and meta-analysis of observational studies. Int J Stroke. 2014;9:1017-1025.

10. Pendlebury ST, Rothwell PM. Prevalence, incidence, and factors associated with pre-stroke and post-stroke dementia: A systematic review and meta-analysis. Lancet Neurol. 2009;8:1006-1018.

11. van Dalen JW, van Charante EPM, Nederkoorn PJ, van Gool WA, Richard E. Poststroke apathy. Stroke. 2013;44:851-860.

12. Pohjasvaara $T$, Vataja $R$, Leppävuori $A$, Kaste $M$, Erkinjuntti $T$. Depression is an independent predictor of poor long-term functional outcome post-stroke. Eur J Neurol 2001;8:315-319.

13. Hama S, Yamashita H, Yamawaki S, Kurisu K. Post-stroke depression and apathy: Interactions between functional recovery, lesion location, and emotional response. Psychogeriatrics. 2011;11:68-76.

14. Santa N, Sugimori H, Kusuda K, Yamashita Y, Ibayashi S, lida M. Apathy and functional recovery following first-ever stroke. Int J Rehabil Res. 2008;31:321-326.

15. Carod-Artal J, Egido JA, González JL, De Seijas EV. Quality of life among stroke survivors evaluated 1 year after stroke. Stroke. 2000;31:2995-3000.

16. Kwok T, Lo RS, Wong E, Wai-Kwong T, Mok V, Kai-Sing W. Quality of life of stroke survivors: A 1-year follow-up study. Arch Phys Med Rehab. 2006;87:1177-1182.

17. Ferro JM, Caeiro L, Figueira ML. Neuropsychiatric sequelae of stroke. Nat Rev Neurol. 2016;12:269280.

18. McKevitt C, Fudge N, Redfern J, Sheldenkar A, Crichton S, Rudd AR, et al. Self-reported long-term needs after stroke. Stroke. 2011;42:1398-1403.

19. Hackett ML, Köhler S, T O'Brien J, Mead GE. Neuropsychiatric outcomes of stroke. Lancet Neurol. 2014;13:525-534.

20. Starkstein SE, Leentjens AF. The nosological position of apathy in clinical practice. J Neurol Neurosurg Psychiatry. 2008;79:1088-1092.

21. Ayerbe L, Ayis $S$, Wolfe CD, Rudd AG. Natural history, predictors and outcomes of depression after stroke: Systematic review and meta-analysis. Br J Psychiatry. 2013;202:14-21. 
22. Köhler S, Verhey F, Weyerer S, Wiese B, Heser K, Wagner M, et al. Depression, non-fatal stroke and all-cause mortality in old age: A prospective cohort study of primary care patients. J Affect Disord. 2013;150:63-69.

23. Bartoli F, Lillia N, Lax A, Crocamo C, Mantero V, Carrà G, et al. Depression after stroke and risk of mortality: A systematic review and meta-analysis. Stroke Res Treat. 2013;2013:1-11.

24. American Psychiatric Association. Diagnostic and statistical manual of mental disorders (DSM-5). Arlington: American Psychiatric Publishing (2013).

25. Coster Ld, Leentjens AF, Lodder J, Verhey FR. The sensitivity of somatic symptoms in post-stroke depression: A discriminant analytic approach. Int J Geriatr Psychiatry. 2005;20:358-362.

26. Cumming T, Churilov L, Skoog I, Blomstrand C, Linden T. Little evidence for different phenomenology in poststroke depression. Acta Psychiatr Scand. 2010;121:424-430.

27. Robinson RG, Szetela B. Mood change following left hemispheric brain injury. Ann Neurol. 1981;9:447-453.

28. Carson AJ, MacHale S, Allen K, Lawrie SM, Dennis M, House A, et al. Depression after stroke and lesion location: A systematic review. Lancet. 2000;356:122-126.

29. Bhogal SK, Teasell R, Foley N, Speechley M. Lesion location and poststroke depression. Stroke. 2004;35:794-802.

30. Wei N, Yong W, Li X, Zhou Y, Deng M, Zhu H, et al. Post-stroke depression and lesion location: A systematic review. J Neurol. 2015;262:81-90.

31. Starkstein SE, Robinson RG, Berthier ML, Parikh RM, Price TR. Differential mood changes following basal ganglia vs thalamic lesions. Arch Neurol. 1988;45:725-730.

32. Morris PL, Robinson RG, Raphael B, Hopwood MJ. Lesion location and poststroke depression. J Neuropsychiatry Clin Neurosci. 1996;8:399-403.

33. Metoki N, Sugawara N, Hagii J, Saito S, Shiroto H, Tomita T, et al. Relationship between the lesion location of acute ischemic stroke and early depressive symptoms in japanese patients. Ann Gen Psychiatry. 2016;15:1-6.

34. Tang WK, Lu JY, Chen YK, Chu WC, Mok V, Ungvari GS, et al. Association of frontal subcortical circuits infarcts in poststroke depression: A magnetic resonance imaging study of 591 chinese patients with ischemic stroke. J Geriatr Psychiatry Neurol. 2011;24:44-49.

35. Hama S, Yamashita H, Shigenobu M, Watanabe A, Kurisu K, Yamawaki S, et al. Post-stroke affective or apathetic depression and lesion location: Left frontal lobe and bilateral basal ganglia. Eur Arch Psychiatry Clin Neurosci. 2007;257:149-152.

36. Tekin S, Cummings JL. Frontal-subcortical neuronal circuits and clinical neuropsychiatry: An update. J Psychosom Res. 2002;53:647-654.

37. Vataja R, Pohjasvaara T, Leppävuori A, Mäntylä R, Aronen H, Salonen O, et al. Magnetic resonance imaging correlates of depression after ischemic stroke. Arch Gen Psychiatry. 2001;58:925-931.

38. Sachdev P, Kalaria R, O'Brien J, Skoog I, Alladi S, Black SE, et al. Diagnostic criteria for vascular cognitive disorders: a VASCOG statement. Alzheimer Dis Assoc Disord. 2014;28:206-218.

39. Vataja R, Pohjasvaara T, Mäntylä R, Ylikoski R, Leskelä M, Kalska H, et al. Depression-executive dysfunction syndrome in stroke patients. Am J Geriatr Psychiatry. 2005;13:99-107.

40. Bour A, Rasquin S, Limburg M, Verhey F. Depressive symptoms and executive functioning in stroke patients: A follow-up study. Int J Geriatr Psychiatry. 2011;26:679-686.

41. Sibolt G, Curtze S, Melkas S, Pohjasvaara T, Kaste M, Karhunen PJ, et al. Post-stroke depression and depression-executive dysfunction syndrome are associated with recurrence of ischaemic stroke. Cerebrovasc Dis. 2013;36:336-343.

42. Melkas S, Vataja R, Oksala NK, Jokinen H, Pohjasvaara T, Oksala A, et al. Depression-executive dysfunction syndrome relates to poor poststroke survival. Am J Geriatr Psychiatry. 2010;18:10071016.

43. Nys G, Van Zandvoort M, Van der Worp H, De Haan E, De Kort P, Kappelle L. Early depressive symptoms after stroke: Neuropsychological correlates and lesion characteristics. J Neurol Sci. 2005;228:27-33. 
44. Zhang $T$, Jing $X$, Zhao X, Wang $C$, Liu Z, Zhou Y, et al. A prospective cohort study of lesion location and its relation to post-stroke depression among chinese patients. J Affect Disord. 2012;136:8387.

45. Robinson R, Jorge R. Post-stroke depression: A review. Am J Psychiat. 2016;173:221-231.

46. Tang WK, Chen YK, Lu JY, Mok VCT, Chu WCW, Ungvari GS, et al. Frontal lobe atrophy in depression after stroke. Stroke Res Treat. 2013;2013:1-4.

47. Tang WK, Chen YK, Lu JY, Chu WCW, Mok VCT, Ungvari GS, et al. Cerebral microbleeds and symptom severity of post-stroke depression: a magnetic resonance imaging study. J Affect Disord. 2011;129:354-358.

48. Tang WK, Chen YK, Lu JY, Chu WCW, Mok VCT, Ungvari GS, et al. White matter hyperintensities in post-stroke depression: a case control study. J Neurol Neurosurg Psychiatry. 2010;81:1312-1315.

49. Kim JT, Park MS, Yoon GJ, Jung HJ, Choi KH, Nam TS, et al. White matter hyperintensity as a factor associated with delayed mood disorders in patients with acute ischemic stroke. Eur Neurol. 2011;66:343-349.

50. Spalletta G, Bossu P, Ciaramella A, Bria P, Caltagirone C, Robinson RG. The etiology of poststroke depression: a review of the literature and a new hypothesis involving infammatory cytokines. Mol Psychiatry. 2006;11:984-91.

51. Kim JM, Stewart R, Bae KY, Kim SW, Kang HJ, Shin IS, et al. Serotonergic and BDNF genes and risk of depression a er stroke. J Affect Disord. 2012;136:833-40.

52. Van Mierlo ML, Van Heugten CM, Post MW, De Kort PL, Visser-Meily JM. Psychological factors determine depressive symptomatology after stroke. Arch Phys Med Rehabil. 2015;96:1064-1070.

53. Aben I, Denollet J, Lousberg R, Verhey F, Wojciechowski F, Honig A. Personality and vulnerability to depression in stroke patients. Stroke. 2002;33:2391-2395.

54. Whyte EM, Mulsant BH. Post stroke depression: epidemiology, pathophysiology, and biological treatment. Biol Psychiatry. 2002;52(3):253-264.

55. Wu S, Barugh A, Macleod M, Mead G. Psychological associations of poststroke fatigue. Stroke. 2014;45:1778-1783.

56. Duncan F, Wu S, Mead GE. Frequency and natural history of fatigue after stroke: A systematic review of longitudinal studies. J Psychosom Res. 2012;73:18-27.

57. Lerdal A, Bakken LN, Rasmussen EF, Beiermann C, Ryen S, Pynten S, et al. Physical impairment, depressive symptoms and pre-stroke fatigue are related to fatigue in the acute phase after stroke. Disabil Rehabil. 2011;33:334-342.

58. Passier P, Post M, van Zandvoort M, Rinkel G, Lindeman E, Visser-Meily J. Predicting fatigue 1 year after aneurysmal subarachnoid hemorrhage. J Neurol. 2011;258:1091-1097.

59. Towfighi A, Ovbiagele B, El Husseini N, Hackett ML, Jorge RE, Kissela BM, et al. Poststroke depression: A scientific statement for healthcare professionals from the american heart association/american stroke association. Stroke. 2016;48:30-43.

60. Aben I, Verhey F. Depression after a cerebrovascular accident. The importance of the integration of neurobiological and psychosocial pathogenic models. Panminerva Med. 2006;48:49-57.

61. De Ryck A, Fransen E, Brouns R, Geurden M, Peij D, Mariën P, et al. Poststroke depression and its multifactorial nature: results from a prospective longitudinal study. J Neurol Sci. 2014;347:159166.

62. Caeiro L, Ferro JM, Costa J. Apathy secondary to stroke: A systematic review and meta-analysis. Cerebrovasc Dis. 2013;35:23-39.

63. Withall A, Brodaty H, Altendorf A, Sachdev PS. A longitudinal study examining the independence of apathy and depression after stroke: The sydney stroke study. Int Psychogeriatr. 2011;23:264273.

64. Mayo NE, Fellows LK, Scott SC, Cameron J, Wood-Dauphinee S. A longitudinal view of apathy and its impact after stroke. Stroke. 2009;40:3299-3307. 
65. Marin RS. Differential diagnosis and classification of apathy. Am J Psychiatry. 1990;147:22-30.

66. Starkstein SE, Petracca G, Chemerinski E, Kremer J. Syndromic validity of apathy in alzheimer's disease. Am J Psychiatry. 2001;158:872-877.

67. Robert P, Onyike C, Leentjens A, Dujardin K, Aalten P, Starkstein S, et al. Proposed diagnostic criteria for apathy in alzheimer's disease and other neuropsychiatric disorders. Eur Psychiatry. 2009;24:98-104.

68. Jorge RE, Starkstein SE, Robinson RG. Apathy following stroke. Can J Psychiatry. 2010;55:350354.

69. Brodaty H, Sachdev PS, Withall A, Altendorf A, Valenzuela MJ, Lorentz L. Frequency and clinical, neuropsychological and neuroimaging correlates of apathy following stroke-the sydney stroke study. Psychol Med. 2005;35:1707-1716.

70. Caeiro L, Ferro JM, Pinho e Melo T, Canhão P, Figueira ML. Post-stroke apathy: An exploratory longitudinal study. Cerebrovasc Dis. 2013;35:507-513.

71. Tagariello P, Girardi P, Amore M. Depression and apathy in dementia: Same syndrome or different constructs? A critical review. Arch Gerontol Geriatr. 2009;49:246-249.

72. Murakami T, Hama S, Yamashita H, Onoda K, Kobayashi M, Kanazawa J, et al. Neuroanatomic pathways associated with poststroke affective and apathetic depression. Am J Geriatr Psychiatry. 2013;21:840-847.

73. Numasawa Y, Hattori T, Ishiai S, Kobayashi Z, Kamata T, Kotera M, et al. Depressive disorder may be associated with raphe nuclei lesions in patients with brainstem infarction. J Affect Disord. 2017;213:191-198.

74. Yang S, Hua P, Shang X, Cui Z, Zhong S, Gong G, et al. A significant risk factor for poststroke depression: The depression-related subnetwork. J Psychiatry Neurosci. 2015;40:259-268.

75. Yang S, Hua P, Shang X, Cui Z, Zhong S, Gong G, et al. Deficiency of brain sutructural sub-network underlying post-ischaemic stroke apathy. Eur J Neurol. 2015;22:341-347.

76. Hackett ML, Anderson CS, House AO, Xia J. Interventions for treating depression after stroke. Stroke. 2009;40:487-488.

77. Levy ML, Cummings JL, Fairbanks LA, Masterman D, Miller BL, Craig AH, et al. Apathy is not depression. J Neuropsychiatry Clin Neurosci. 1998;10:314-319.

78. Wongpakaran N, van Reekum R, Wongpakaran T, Clarke D. Selective serotonin reuptake inhibitor use associates with apathy among depressed elderly: A case-control study. Ann Gen Psychiatry. 2007;6:1-6. 
CHAPTER 2 


\section{IMAGING MARKERS OF POST-STROKE DEPRESSION AND APATHY: A SYSTEMATIC REVIEW AND META-ANALYSIS}

Elles Douven

Sebastian Köhler

Maria MF Rodriguez

Julie Staals

Frans RJ Verhey

Pauline Aalten 


\section{ABSTRACT}

Several brain imaging markers have been studied in the development of post-stroke depression (PSD) and post-stroke apathy (PSA), but inconsistent associations have been reported. This systematic review and meta-analysis aims to provide a comprehensive and up-to-date evaluation of imaging markers associated with PSD and PSA. Databases (Medline, Embase, PsycINFO, CINAHL, and Cochrane Database of Systematic Reviews) were searched from inception to July 21, 2016. Observational studies describing imaging markers of PSD and PSA were included. Pooled odds ratios (OR) and 95\% confidence intervals $(\mathrm{Cl})$ were calculated to examine the association between PSD or PSA and stroke lesion laterality, type, and location, also stratified by study phase (acute, post-acute, chronic). Other imaging markers were reviewed qualitatively. The search retrieved 4502 studies, of which 149 studies were included in the review and 86 studies in the metaanalyses. PSD in the post-acute stroke phase was significantly associated with frontal (OR 1.72, 95\% Cl 1.34-2.19) and basal ganglia lesions (OR 2.25, $95 \% \mathrm{Cl} 1.33-3.84)$. Hemorrhagic stroke related to higher odds for PSA in the acute phase (OR 2.58,95\% Cl 1.18-5.65), whereas ischemic stroke related to higher odds for PSA in the post-acute phase (OR 0.20, 95\% Cl 0.06-0.69). Frequency of PSD and PSA is modestly associated with stroke type and location and is dependent on stroke phase. These findings have to be taken into consideration for stroke rehabilitation programs, as this could prevent stroke patients from developing PSD and PSA, resulting in better clinical outcome. 


\section{INTRODUCTION}

Post-stroke depression (PSD) and post-stroke apathy (PSA) are frequent neuropsychiatric symptoms after stroke, with estimated prevalence rates between $30 \%$ and $40 \%$, respectively, in the first few months after stroke. ${ }^{1}$ Depression can be defined as a feeling of low mood, loss of interest, and lack of pleasure that persists for a time period of at least 2 weeks. ${ }^{2}$ Apathy is generally defined as a disorder of diminished motivation, characterized by loss of interest, diminished emotional response, and loss of initiative, ${ }^{3}$ and can occur independently, ${ }^{4}$ or in combination with symptoms of depression. ${ }^{5}$

According to a previous meta-analysis, approximately $40 \%$ of patients with PSA also suffer from PSD. ${ }^{6}$ Because depression and apathy share several features, mainly loss of interest, patients with apathy after stroke are frequently misdiagnosed as having PSD. ${ }^{7}$ However, despite the considerable overlap in symptoms between PSD and PSA, there is evidence indicating that the two syndromes seem to develop from different anatomical and neurobiological constructs. . $^{8-12}$

Earlier studies have already attempted to disentangle the relationship between PSD and PSA, which have shown inconclusive results. The Sydney Stroke Study showed evidence for independence of PSA and PSD when measuring 3 to 6 months poststroke. ${ }^{13}$ However, at 1 -year follow-up, a significant overlap between apathy and depression was found. ${ }^{14}$ Contrastingly, Caeiro et al. ${ }^{15}$ did not find an association between PSA and PSD at 1-year post-stroke. It is important to disentangle the relationship between PSA and PSD, at least from a clinical perspective, since the two syndromes seem to benefit from different types of medication. ${ }^{14}$ Both PSD and PSA are known to have a negative influence on clinical outcome $e^{16,17}$ and quality of life. ${ }^{18,19}$ Early treatment and prevention of PSD and PSA might have a positive effect on functional outcome, thereby limiting the impact of stroke on patients' daily lives. ${ }^{20}$ Identification of associated risk factors is thus important for early detection and tailoring of rehabilitation programs.

Several brain imaging markers have been studied in the development of PSD. Early studies suggested that PSD is frequent in patients with left frontal lesions, ${ }^{9,13}$ but this hypothesis could not be supported by later studies. ${ }^{21}$ Previous systematic reviews primarily looked at the relationship with lesion laterality, while other potential imaging markers such as lesion location, lesion type, lesion volume, white matter hyperintensities (WMH), and atrophy have been ignored. ${ }^{21-23}$ In addition, imaging markers of PSA have been studied less frequently compared with PSD. Some studies provided evidence that PSA is associated with right hemispheric and subcortical lesions, ${ }^{8,24,25}$ though two recent systematic reviews on lesion location in PSA reported inconclusive results. ${ }^{6,26}$ Caeiro et al. ${ }^{26}$ only studied the association 
with lesion laterality and were not able to find an association, whereas van Dalen et al. ${ }^{6}$ provided a qualitative overview of associations with lesion location and laterality, but no meta-analysis, and concluded that no clear association with lesion side or location could be found, though associations with the basal ganglia were most consistent.

A systematic review and meta-analysis was performed to evaluate the association between different brain imaging markers and PSD and PSA, thereby updating and extending previous meta-analyses just focusing on lesion location in association with PSD and PSA. The main aim of the present study was to investigate differences and similarities in several brain correlates associated with PSD and PSA.

Post-stroke depression (PSD) and post-stroke apathy (PSA) are frequent neuropsychiatric symptoms after stroke, with estimated prevalence rates between $30 \%$ and $40 \%$, respectively, in the first few months after stroke. ${ }^{1}$ Depression can be defined as a feeling of low mood, loss of interest, and lack of pleasure that persists for a time period of at least 2 weeks. ${ }^{2}$ Apathy is generally defined as a disorder of diminished motivation, characterized by loss of interest, diminished emotional response, and loss of initiative, ${ }^{3}$ and can occur independently, ${ }^{4}$ or in combination with symptoms of depression. ${ }^{5}$

According to a previous meta-analysis, approximately $40 \%$ of patients with PSA also suffer from PSD. ${ }^{6}$ Because depression and apathy share several features, mainly loss of interest, patients with apathy after stroke are frequently misdiagnosed as having PSD. ${ }^{7}$ However, despite the considerable overlap in symptoms between PSD and PSA, there is evidence indicating that the two syndromes seem to develop from different anatomical and neurobiological constructs. ${ }^{8-12}$

Earlier studies have already attempted to disentangle the relationship between PSD and PSA, which have shown inconclusive results. The Sydney Stroke Study showed evidence for independence of PSA and PSD when measuring 3 to 6 months poststroke. ${ }^{13}$ However, at 1-year follow-up, a significant overlap between apathy and depression was found. ${ }^{14}$ Contrastingly, Caeiro et al. ${ }^{15}$ did not find an association between PSA and PSD at 1-year post-stroke. It is important to disentangle the relationship between PSA and PSD, at least from a clinical perspective, since the two syndromes seem to benefit from different types of medication. ${ }^{14}$ Both PSD and PSA are known to have a negative influence on clinical outcome ${ }^{16,17}$ and quality of life. ${ }^{18,19}$ Early treatment and prevention of PSD and PSA might have a positive effect on functional outcome, thereby limiting the impact of stroke on patients' daily lives. ${ }^{20}$ Identification of associated risk factors is thus important for early detection and tailoring of rehabilitation programs. 
Several brain imaging markers have been studied in the development of PSD. Early studies suggested that PSD is frequent in patients with left frontal lesions, 9,13 but this hypothesis could not be supported by later studies. ${ }^{21}$ Previous systematic reviews primarily looked at the relationship with lesion laterality, while other potential imaging markers such as lesion location, lesion type, lesion volume, white matter hyperintensities (WMH), and atrophy have been ignored. ${ }^{21-23}$ In addition, imaging markers of PSA have been studied less frequently compared with PSD. Some studies provided evidence that PSA is associated with right hemispheric and subcortical lesions, ${ }^{8,24,25}$ though two recent systematic reviews on lesion location in PSA reported inconclusive results. ${ }^{6,26}$ Caeiro et al. ${ }^{26}$ only studied the association with lesion laterality and were not able to find an association, whereas van Dalen et al. ${ }^{6}$ provided a qualitative overview of associations with lesion location and laterality, but no meta-analysis, and concluded that no clear association with lesion side or location could be found, though associations with the basal ganglia were most consistent.

A systematic review and meta-analysis was performed to evaluate the association between different brain imaging markers and PSD and PSA, thereby updating and extending previous meta-analyses just focusing on lesion location in association with PSD and PSA. The main aim of the present study was to investigate differences and similarities in several brain correlates associated with PSD and PSA.

\section{METHODS}

\section{SEARCH STRATEGY AND SELECTION CRITERIA}

This systematic review and meta-analysis was conducted according to the Preferred Reporting Items for Systematic Reviews and Meta-Analyses (PRISMA) statement ${ }^{27}$ and by use of a predefined research protocol. Databases (Medline, Embase, PsycINFO, CINAHL, and Cochrane Database of Systematic Reviews) were searched from inception to December 2015 and updated to July 21, 2016. A full description of the search strategy is presented in supplementary Online Resource 1. To be eligible for inclusion, studies had to a) include patients with ischemic or hemorrhagic stroke, b) assess the presence of depressive or apathetic symptoms, c) examine the association between these symptoms and an imaging marker, $d$ ) the population had to be human adults, e) sample size had to be larger than 25 to avoid inclusion of spurious associations from underpowered studies, and $f$ ) language had to be English, German, Dutch, or French. Studies were excluded if (a) the study population was other than stroke or a combined population was studied without separate results available for stroke, (b) the population consisted 
of only patients with cognitive impairment or dementia in which vascular damage (infarcts, WMH, atrophy) was studied, or (c) no imaging data or lesion-related data (e.g. lesion location, type, laterality, WMH) were described. Records of research protocols, reviews, and abstracts from scientific meetings were excluded. If studies presented results from the same cohort on a certain outcome measure, data from the study that used the largest group of patients were used, or if they used an equal number of patients, data from the earliest publication were used.

Two reviewers (E.D. and P.A.) independently screened titles and abstracts manually for potential eligibility. Doubtful records were discussed (E.D. and P.A.) and an independent third reviewer (S.K.) decided if doubtful cases were included or not for full-text scrutiny. For completeness, reference lists were screened for additional articles. One reviewer (E.D.) assessed eligibility for inclusion based on full-text screening.

\section{DATA COLLECTION AND EXTRACTION}

Data extraction was performed on selected articles for which full texts were obtained. Two independent reviewers (E.D. and M.R.) extracted data from each study according to a predefined data extraction form. The following information was extracted for each study included in the review: (1) first author and year of publication, (2) demographic characteristics, (3) in- and exclusion criteria if specified, (4) imaging method and imaging markers, (5) questionnaires and criteria used to define PSD or PSA, (6) time of measurement after stroke, (7) statistical methods used and results needed for the meta-analysis, (8) main conclusion and limitations.

\section{STATISTICAL ANALYSES AND STUDY QUALITY}

Statistical analyses were performed using STATA 13.1 (StataCorp, TX, USA). Statistical significance was defined by $p<.05$ in two-sided tests. Pooled odds ratios (ORs) with corresponding 95\% confidence intervals ( $\mathrm{Cls}$ ) were calculated to examine the association between PSD or PSA and stroke lesion laterality, type, and location, also stratified by study phase (acute, post-acute, chronic), using a DerSimonian-Laird random-effects model to account for within- and betweenstudy variance. ${ }^{28} \mathrm{~A}$ full description of the observations used in the meta-analyses is presented in supplementary Online Resource 2. Studies were stratified according to phase in which they measured depression or apathy after stroke: acute (< 15 days from stroke onset), post-acute (15 days - 6 months), and chronic phase ( $>6$ months). This stratification was based on the meta-analysis by Caeiro et al. ${ }^{26}$ and was chosen because the acute stroke phase corresponds with the period 
of hospitalization and acute care ${ }^{29}$ and in this period the risk of complications and recurrent stroke is highest. ${ }^{30}$ After this period of acute care, patients usually start with rehabilitation and most recovery will take place in the first six months. ${ }^{31}$ Therefore, we defined this period as the post-acute period, and $>6$ months as the chronic stroke phase.

To identify possible sources of heterogeneity, random-effect meta-regression models were conducted including the following covariates: study phase, mean age, PSD/PSA prevalence, percentage of females, imaging method (CT/MRI vs. $M R I)$, patient source, and first-ever stroke (yes/no).

The study quality was assessed by a single investigator (E.D.) with the NewcastleOttowa Scale (NOS) for case-control and cohort studies, and a modified NOS was applied for cross-sectional studies. ${ }^{32}$ A maximum score of 9 can be obtained and studies with a score $<5$ were not included in the meta-analyses (see supplementary Online Resource 3). Visual inspection of asymmetry in funnel plots, in which the relation between sample size and effect size is assessed, was used to test for possible publication bias. Egger's regression tests were performed to test for significant asymmetry of funnel plots as a test for small-study effects. ${ }^{33}$

\section{RESULTS}

Of 4502 identified articles, 167 articles were selected for full-text screening (see Figure 1). Nine articles could not be retrieved from authors after several contact requests. Based on full-text evaluation of the remaining articles, 135 articles met inclusion criteria. Reasons for exclusion were: conference abstract, ${ }^{34}$ research protocol, ${ }^{35}$ review article, ${ }^{36,37}$ small sample size, ${ }^{38-45}$ other outcome than depression or apathy, ${ }^{46-48}$ study population other than stroke or no separate results available for stroke subpopulation, ${ }^{49-55}$ and no evaluation of imaging markers. ${ }^{56}$ Fourteen additional studies found in reference lists and fulfilling eligibility criteria were included, resulting in a total of 149 studies.

\section{CHARACTERISTICS OF INCLUDED STUDIES}

A detailed overview of study characteristics for PSD studies $(n=132)$ and PSA studies $(n=23$ ) is presented in supplementary Online Resource 4 and 5 . Of all PSD studies, 51 (39\%) studies included only first-ever strokes. Thirty-nine cohorts (30\%) were followed prospectively. Some studies used semi-structured psychiatric interviews like the Mini International Neuropsychiatric Interview, ${ }^{57}$ or the Structured Clinical Interview for DSM disorders, ${ }^{58}$ based on Diagnostic and Statistical Manual of Mental Disorders (DSM) version $1{ }^{59}$ or IV, ${ }^{60}$ whereas others 
used clinician-rated or self-rated questionnaires (e.g. the Hamilton Depression Rating Scale, ${ }^{61}$ Montgomery-Åsberg Depression Rating Scale, ${ }^{62}$ or the Geriatric Depression Scale ${ }^{63}$ to evaluate the presence of PSD, and different cut-offs were applied.

Based on 107 (81\%) studies that reported on PSD prevalence within the first year, a median prevalence of $30.4 \%$ was found (IQR 20.1-40.0). Of all PSA studies, nine (39\%) studies included first-ever stroke patients. Four (17\%) cohorts were studied prospectively. Most studies used the Apathy Scale ${ }^{64}$ or Apathy Evaluation Scale ${ }^{65}$ to evaluate the presence of PSA, and different cut-offs were applied. Based on 20 (87\%) studies that reported on PSA prevalence within the first year, a median prevalence of $37.3 \%$ was found (IQR 22.1-42.5).

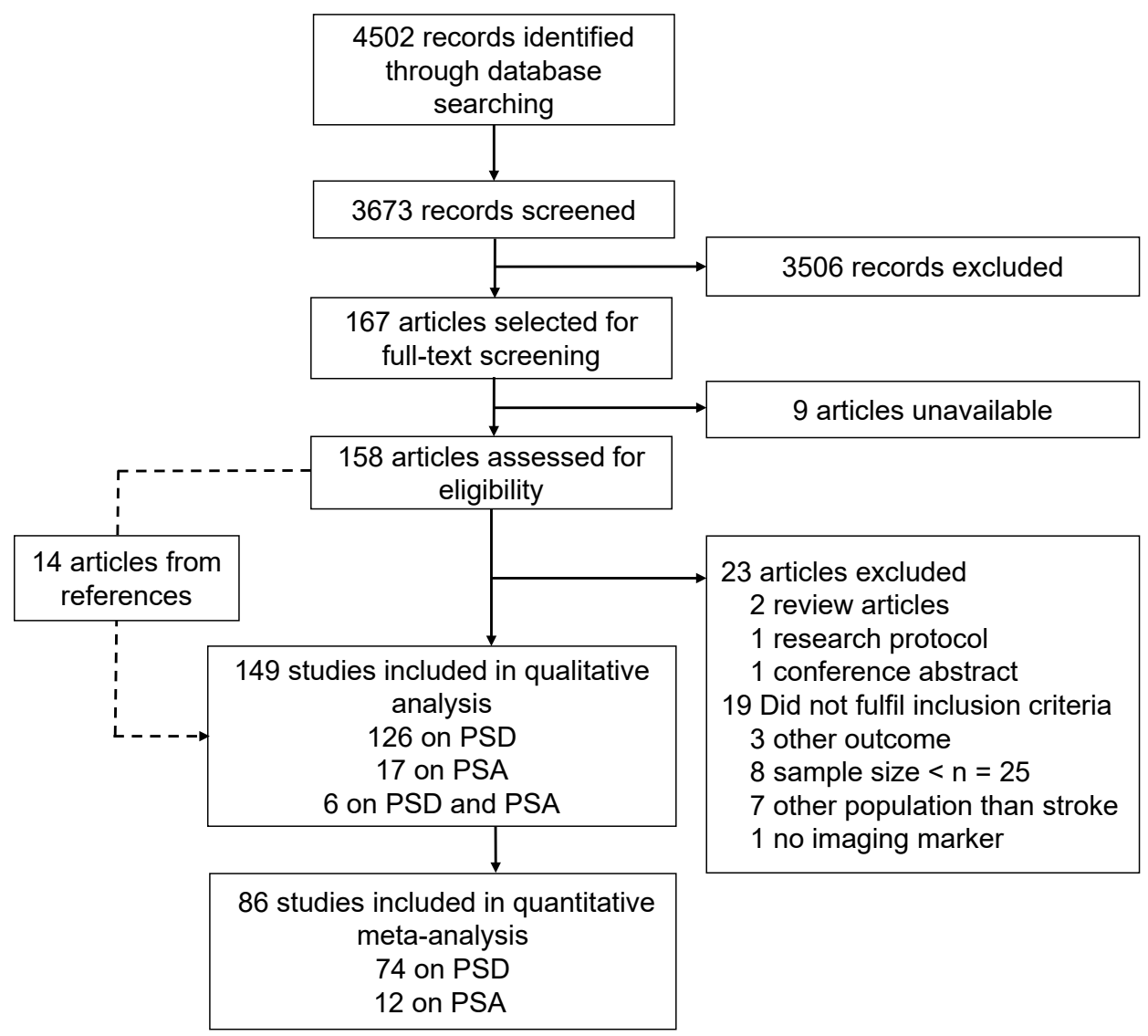

Figure 1. Preferred Reporting Items for Systematic Reviews and Meta-analyses (PRISMA) flowchart of study selection and review

Abbreviations: PSA, post-stroke apathy; PSD, post-stroke depression 


\section{LESION LATERALITY}

Sixty (45\%) studies presented data on PSD and lesion laterality. In the pooled analyses, no significant overall association between PSD and lesion side was found (Table 1). A subgroup analysis stratified by study phase showed a $26 \%$ higher odds of PSD after left-sided stroke in the acute phase, but this effect was not statistically significant (OR $1.26,95 \% \mathrm{Cl} 0.95-1.67, l^{2}=60.1 \%$, see Figure 2 ). Neither in the post-acute stroke phase (OR $1.00,95 \% \mathrm{Cl} 0.83-1.20, l^{2}=50.4 \%$ ), nor in the chronic stroke phase (OR $1.12, \mathrm{Cl} 0.87-1.45, \mathrm{I}^{2}=0.0 \%$ ) a significant association was found with lesion side (see Figure 3).

Nine (39\%) studies presented data on PSA and lesion laterality. In the pooled analyses, the overall odds of PSA were a bit higher after left-sided stroke (Table 1). A subgroup analysis stratified by study phase showed higher odds after left-sided stroke in the post-acute phase, although this effect was not statistically significant (OR 1.90, 95\% Cl 0.88-4.09, $l^{2}=0.0 \%$, see Figure $4 \mathrm{~A}$ ). No significant association was found in the acute stroke phase (OR $0.95,95 \% \mathrm{Cl} 0.42-2.16, I^{2}=72.0 \%$, see Figure $4 \mathrm{~A}$ ) and no studies reported on the association in the chronic phase.

Table 1. Overall effect sizes and Egger's bias coefficients

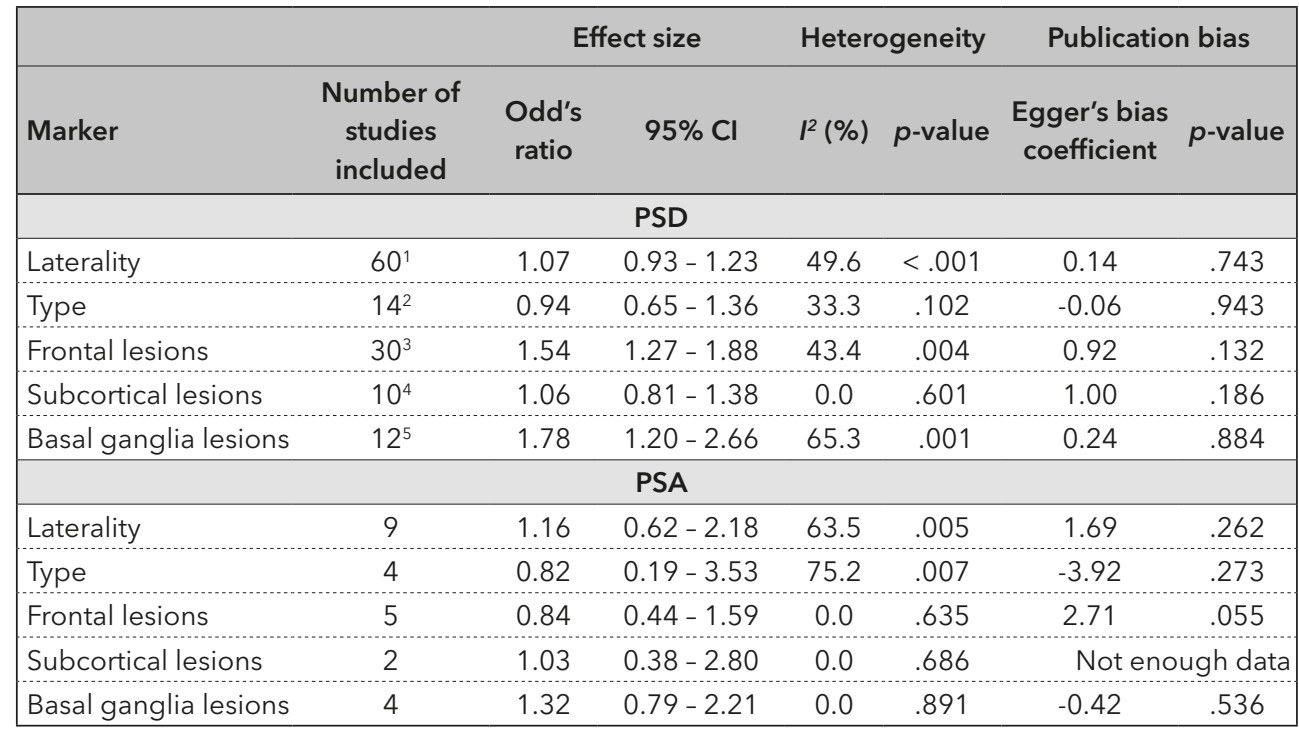

Abbreviations: $\mathrm{Cl}$, confidence interval; PSA, post-stroke apathy; PSD, post-stroke depression.

Note: ${ }^{1}$ Six of the 60 studies provided data on more than one time point. ${ }^{2}$ One of the 14 studies provided data on more than one time point. ${ }^{3}$ Five of the 30 studies provided data on more than one time point. ${ }^{4}$ Two of the 10 studies provided data on more than one time point. ${ }^{5}$ One of the 12 studies provided data on more than one time point. 


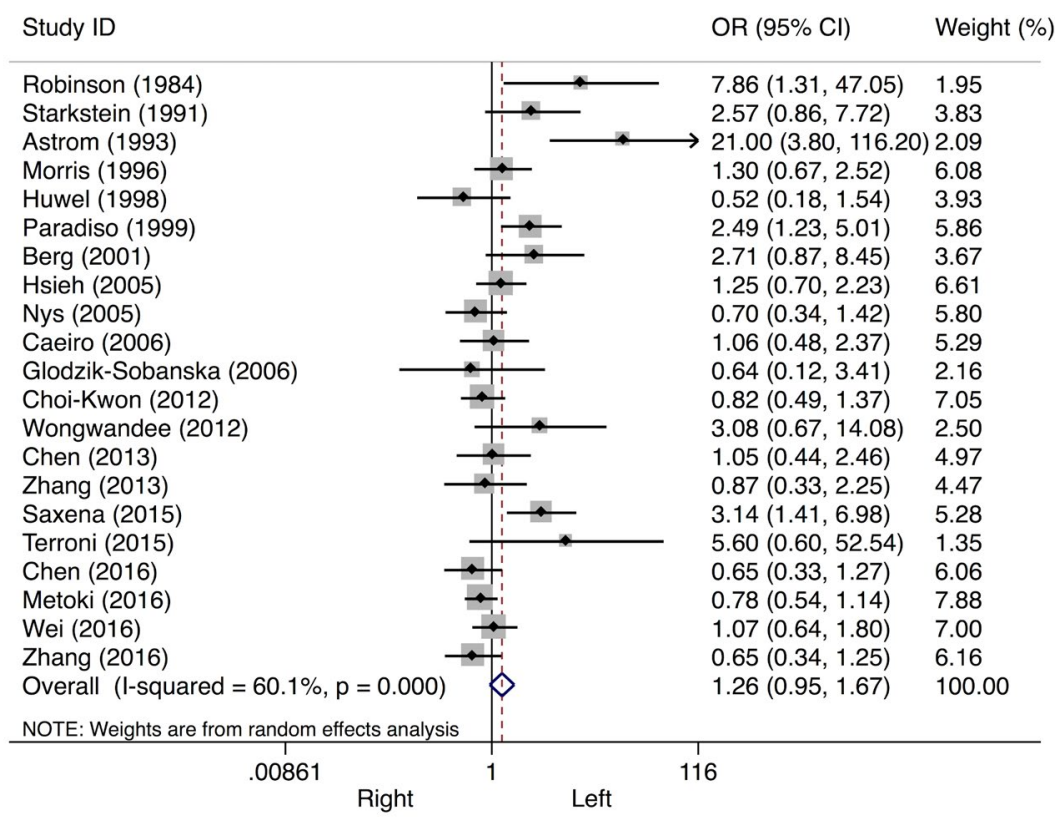

Figure 2. Forest plot of the relationship between post-stroke depression and lesion laterality Note: Subanalyses on acute stroke phase are presented.

Abbreviations: $\mathrm{Cl}$, confidence interval; $\mathrm{OR}$, odds ratio.

\section{LESION TYPE}

Fourteen (11\%) studies reported outcomes on lesion type associated with PSD. Overall, no significant association between PSD and lesion type was observed (Table 1). A subgroup analysis by study phase showed no significant association between lesion type and PSD in the acute (OR 0.95, 95\% $\mathrm{Cl} 0.59-1.53, \mathrm{I}^{2}=14.0 \%$ ), post-acute (OR $\left.0.94,95 \% \mathrm{Cl} 0.47-1.87, \mathrm{I}^{2}=59.9 \%\right)$, or chronic stroke phase (OR $0.76,95 \% \mathrm{Cl} 0.22-2.65, l^{2}=0.0 \%$, see Figure 5 ).

Four (17\%) studies reported outcomes on lesion type associated with PSA. Overall, the odds of PSA after hemorrhagic stroke was not higher than after ischemic stroke (Table 1). A subgroup analysis by study phase showed higher odds after hemorrhagic stroke in the acute phase (OR $2.58,95 \% \mathrm{Cl} 1.18-5.65, \mathrm{I}^{2}=0.0 \%$, see Figure $4 \mathrm{~B})$, whereas higher odds after ischemic stroke were found in the postacute phase (OR $0.20,95 \% \mathrm{Cl} 0.06-0.69,1^{2}=0.0 \%$, see Figure 4B). Only two studies were included per phase. 


\section{Post-acute}

Eastwood (1989)

House (1990)

Morris (1990)

Astrom (1993)

Schwartz (1993)

Andersen (1995)

Gonzàlez-Torrecillas (1995)

Herrmann (1995)

$\mathrm{Ng}$ (1995)

Bendsen (1997)

Kase (1998)

MacHale (1998)

Pohjasvaara (1998)

Paolucci (1999)

Kim (2000)

Singh (2000)

Desmond (2003)

Spalletta (2005)

Glodzik-Sobanska (2006)

Wichowicz (2006)

Brodaty (2007)

Fuentes (2009)

Oladiji (2009)

Snaphaan (2009)

Bour (2010)

Nishiyama (2010)

Sienkiewicz-Jarosz (2010)

Tang (2010)

Tennen (2011)

Terroni (2011)

Altieri (2012)

Choi-Kwon (2012)

Zhang (2012)

Rajashekaran (2013)

Gozzi (2014)

Jiang (2014)

Wichowicz (2015)

Chen (2016)

Wei (2016)

Subtotal (I-squared $=50.4 \%, p=0.000$ )

Chronic

House (1990)

Sharpe (1990)

Astrom (1993)

Provinciali (2008)

Chatterjee (2010)

Nidhihandana (2010)

Srivastava (2010)

Subtotal (I-squared $=0.0 \%, p=0.725$ )

Overall (I-squared $=44.1 \%, p=0.001$ )

NOTE: Weights are from random effects analysis

.02

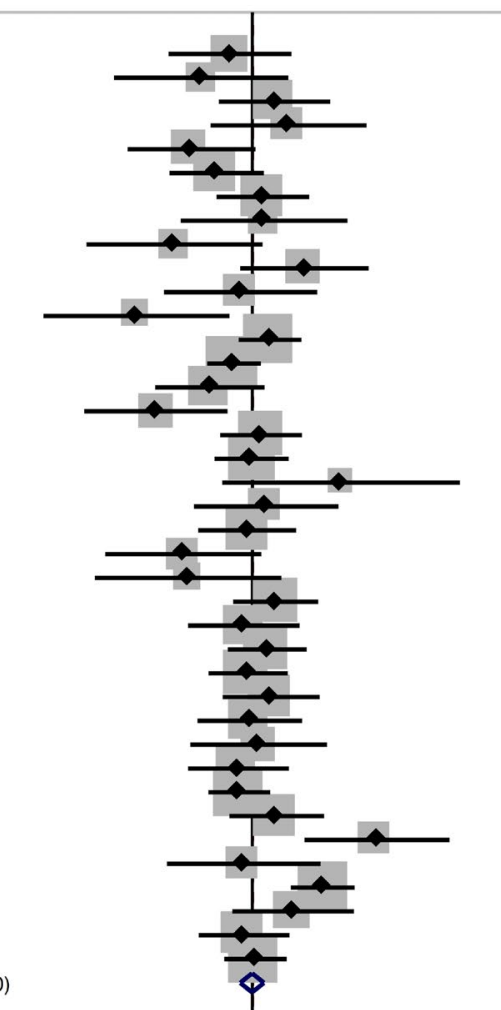

$0.71(0.27,1.83)$

$0.45(0.12,1.74) \quad 1.13$

$1.41(0.59,3.33) \quad 2.15$

$1.75(0.52,5.84)$

$0.39(0.15,1.05) \quad 1.79$

$0.58(0.28,1.19)$

$1.18(0.58,2.40)$

$1.20(0.33,4.36)$

$0.30(0.08,1.17)$

$2.24(0.83,6.04) \quad 1.78$

$0.83(0.25,2.72)$

$0.17(0.04,0.70)$

$1.31(0.81,2.14) \quad 3.75$

$0.75(0.50,1.14) \quad 4.14$

$0.52(0.22,1.21)$

$0.22(0.07,0.68)$

$1.14(0.61,2.15)$

$0.99(0.56,1.76) \quad 3.30$

$3.94(0.63,24.73) \quad 0.67$

$1.24(0.41,3.78)$

$0.92(0.43,1.97) \quad 2.50$

$0.34(0.10,1.15)$

$0.37(0.09,1.56) \quad 1.01$

$1.43(0.74,2.77)$

$0.88(0.37,2.08)$

$1.26(0.68,2.32) \quad 3.12$

$0.94(0.51,1.73) \quad 3.11$

$1.34(0.63,2.82)$

$0.96(0.43,2.16)$

$1.10(0.38,3.17)$

$0.81(0.37,1.76) \quad 2.42$

$0.82(0.51,1.32) \quad 3.78$

$1.46(0.70,3.03) \quad 2.60$

$6.86(2.24,21.05)$

$0.88(0.27,2.87)$

$2.97(1.81,4.86) \quad 3.71$

$1.88(0.73,4.80)$

$0.88(0.44,1.78) \quad 2.73$

$1.05(0.65,1.70)$

$1.00(0.83,1.20) \quad 86.66$

$0.19(0.02,1.83) \quad 0.46$

$1.15(0.31,4.28) \quad 1.18$

$1.17(0.26,5.21) \quad 0.96$

$1.19(0.88,1.62) \quad 4.76$

$1.44(0.62,3.33) \quad 2.22$

$0.82(0.37,1.84) \quad 2.33$

$0.85(0.27,2.69)$

$1.12(0.87,1.45) \quad 13.34$

$1.01(0.86,1.18) \quad 100.00$

Right Left

Figure 3. Forest plot of the relationship between post-stroke depression and lesion laterality Note: Subanalyses on post-acute stroke phase (upper panels) and chronic stroke phase (lower panels) are presented.

Abbreviations: $\mathrm{Cl}$, confidence interval; $\mathrm{OR}$, odds ratio. 


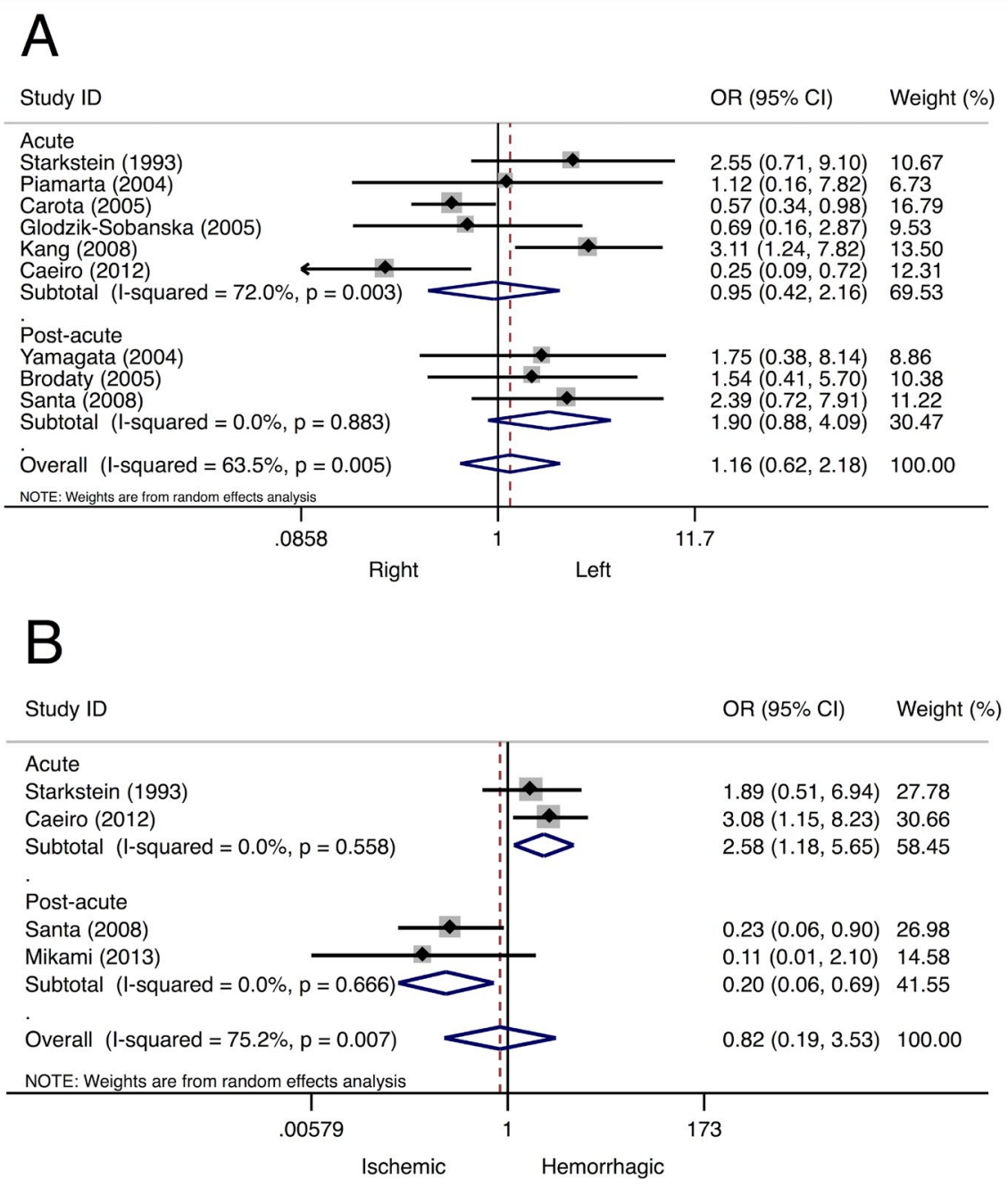

Figure 4. Forest plot of the relationship between post-stroke apathy and lesion laterality/type Note: In panel A, the results of the meta-analysis on lesion laterality are presented. In panel B, the results of the meta-analysis on lesion type are presented. Apart from the overall analysis, the subanalyses on acute stroke phase (upper panels) and post-acute stroke phase (lower panels) are presented. Abbreviations: $\mathrm{Cl}$, confidence interval; $\mathrm{OR}$, odds ratio. 


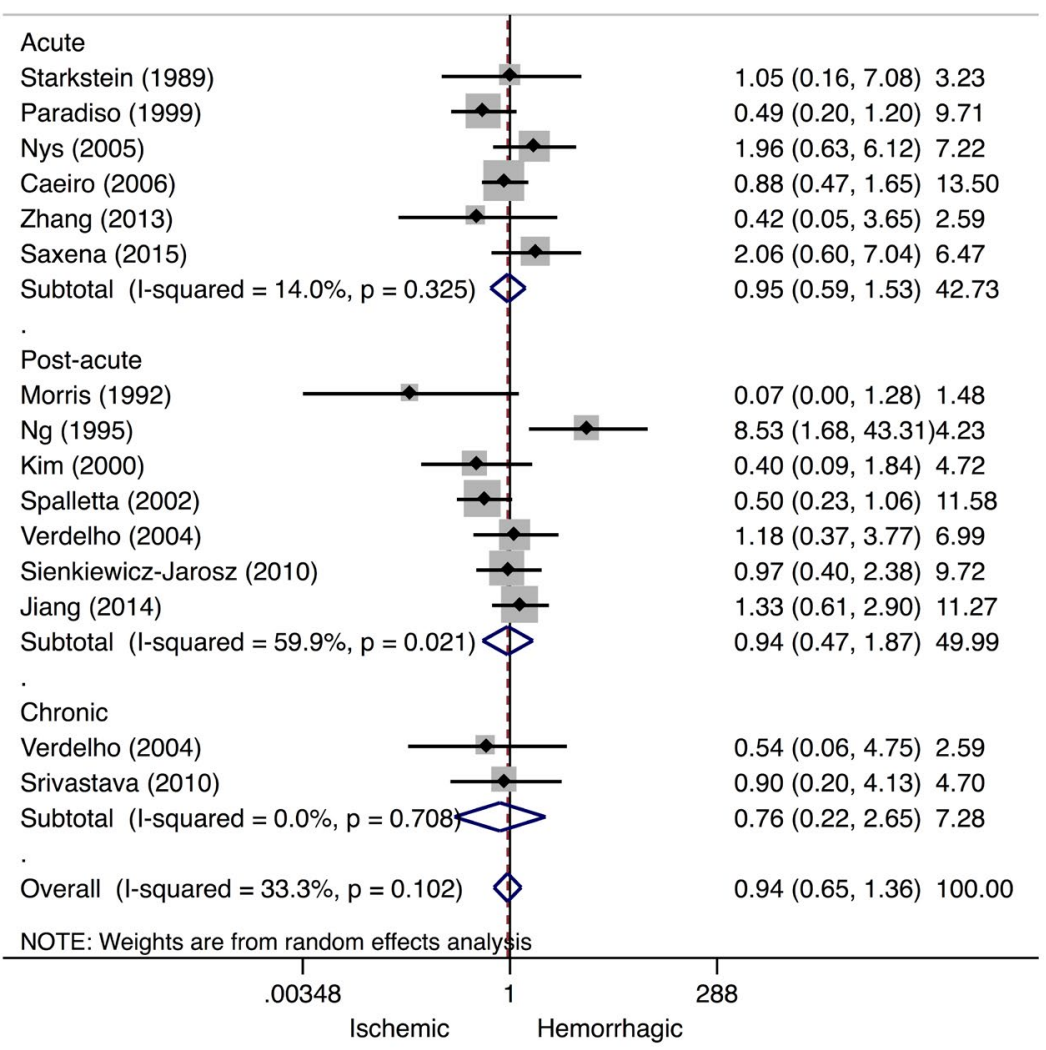

Figure 5. Forest plot of the relationship between post-stroke depression and lesion type Note: Apart from the overall analysis, the subanalyses on acute stroke phase (upper panels), post-acute stroke phase (middle panels), and chronic stroke phase (lower panels) are presented. Abbreviations: $\mathrm{Cl}$, confidence interval; $\mathrm{OR}$, odds ratio. 


\section{LESION LOCATION}

In Table 2, an overview is provided of lesion locations that were significantly associated with PSD. As frontal/anterior, subcortical, and basal ganglia lesions were frequently associated with PSD, meta-analyses were performed on these locations. Thirty (23\%) studies reported outcomes on frontal lesion location associated with PSD. Overall, a $54 \%$ higher odds of PSD after frontal stroke was found (Table 1). Subgroup analysis suggested this association was limited to PSD in the post-acute stroke phase (OR 1.72, 95\% Cl 1.34-2.19, $I^{2}=47.2 \%$ ), as no significant association was found in the acute stroke phase (OR $1.21,95 \% \mathrm{Cl} 0.90-1.63, I^{2}=21.1 \%$ ), see Figure 6.

Ten (8\%) studies reported outcomes on subcortical lesion location associated with PSD. Pooled odds for PSD were not significantly higher after subcortical lesions (Table 1). A subgroup analysis by study phase showed no significant associations between subcortical lesions and PSD in the acute (OR 1.04, 95\% Cl 0.64-1.70), post-acute (OR $0.93,95 \% \mathrm{Cl} 0.65-1.32$ ), or chronic stroke phase (OR $1.88,95 \% \mathrm{Cl}$ 0.92-3.84), see Figure 7A, but the latter association consisted only of two studies. No significant heterogeneity was observed (each phase, $l^{2}=0.0 \%$ ). Twelve (9\%) studies reported outcomes on basal ganglia lesion location associated with PSD. Overall, basal ganglia lesions were significantly associated with PSD (Table 1). A subgroup analysis by study phase showed that basal ganglia lesions were significantly associated with PSD in the post-acute phase (OR 2.25, 95\% Cl 1.33$3.84,1^{2}=71.2 \%$ ), but not in the acute stroke phase (OR $1.26,95 \% \mathrm{Cl} 0.74-2.14,1^{2}$ $=41.4 \%$ ), see Figure 7B.

Five (22\%) studies provided data on the association between PSA and frontal lesions. Overall, no significant association between PSA and frontal lesions was found (Table 1). Subgroup analyses showed different albeit no significant results per phase, with stronger associations with frontal lesions in the acute phase (OR $\left.1.68,95 \% \mathrm{Cl} 0.52-5.45, I^{2}=0.0 \%\right)$, and an inverse relation in the post-acute phase (OR $0.63,95 \% \mathrm{Cl} 0.29-1.34, l^{2}=0.0 \%$ ), see Figure $8 \mathrm{~A}$. No significant association between PSA and subcortical lesions was found (Table 1), but this was only evaluated in two (9\%) studies (OR $1.03,95 \% \mathrm{Cl} 0.38-2.80, l^{2}=0.0 \%$ ), see Figure $8 \mathrm{~B}$. Four (17\%) studies provided data on the association between PSA and basal ganglia lesions. Overall, no significant association between PSA and basal ganglia lesions was found (Table 1). Stratification by study phase showed similar results, with no significant heterogeneity (acute phase: OR $1.45,95 \% \mathrm{Cl} 0.42-4.95$, postacute phase: OR 1.29, 95\% Cl 0.73-2.29), see Figure 8C. 


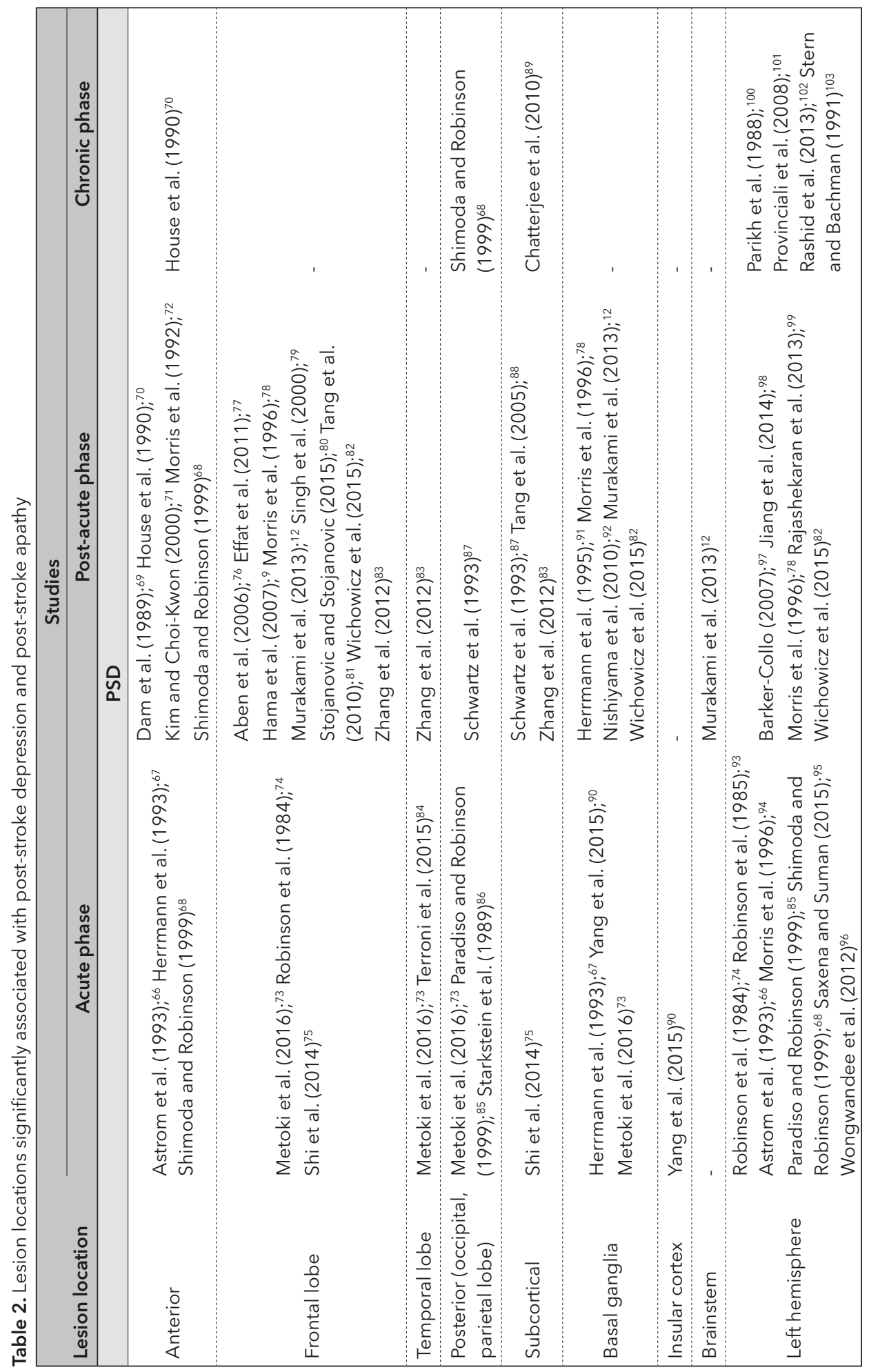




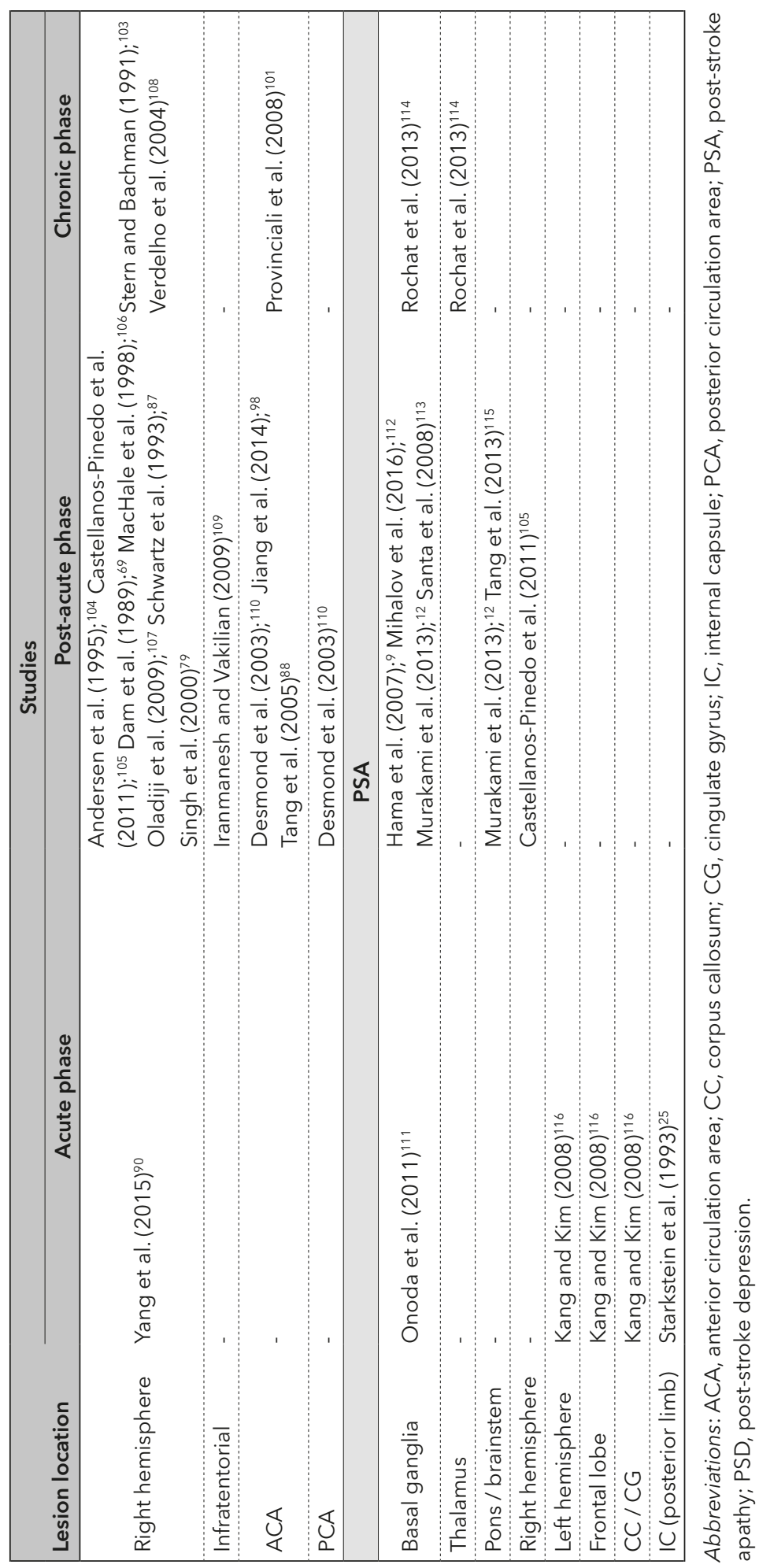




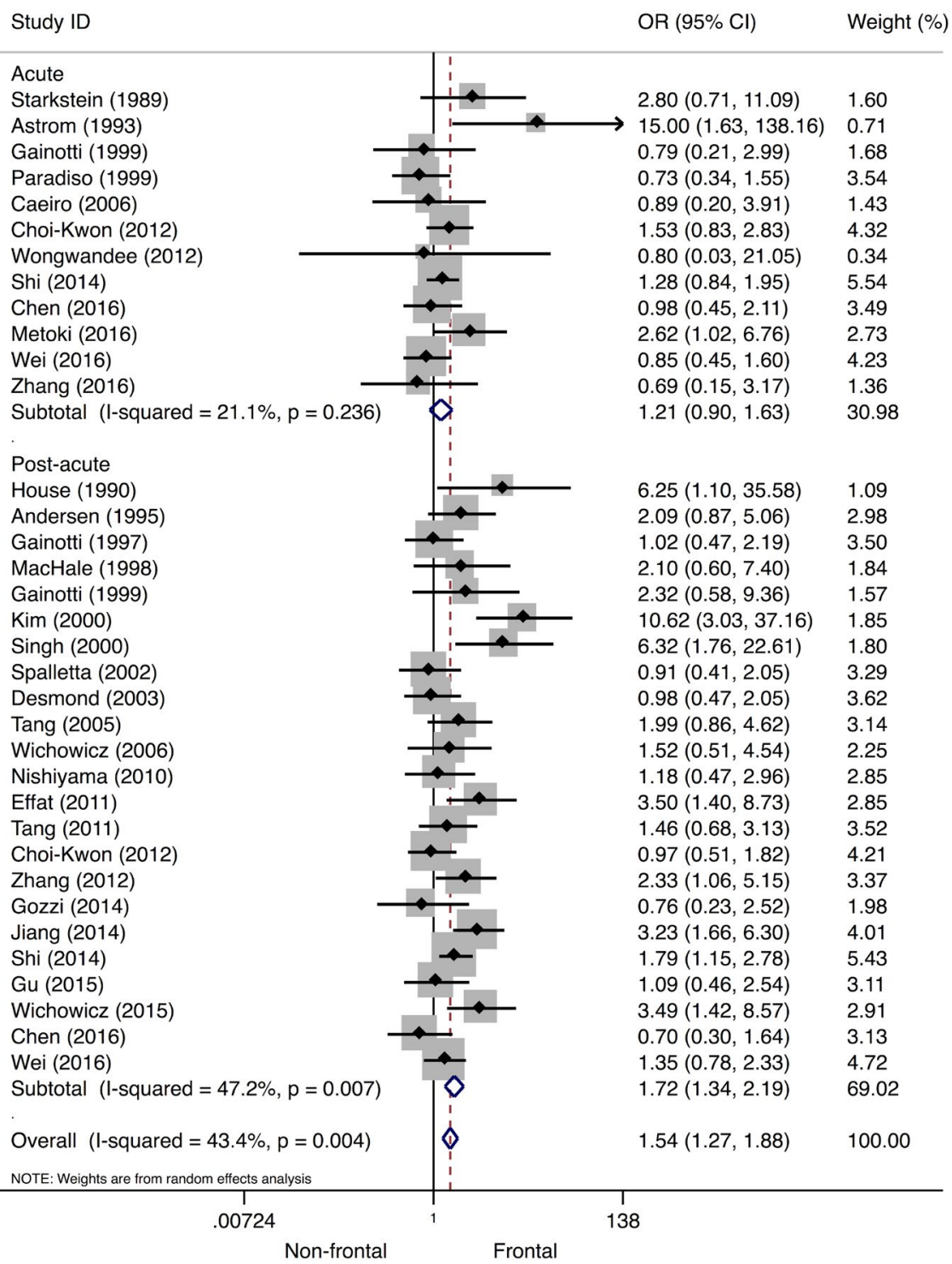

Figure 6. Forest plot of the relationship between post-stroke depression and frontal/anterior lesions Note: Apart from the overall analysis, the subanalyses on acute stroke phase (upper panels) and postacute stroke phase (lower panels) are presented. Abbreviations: $\mathrm{Cl}$, confidence interval; $\mathrm{OR}$, odds ratio 


\section{A}

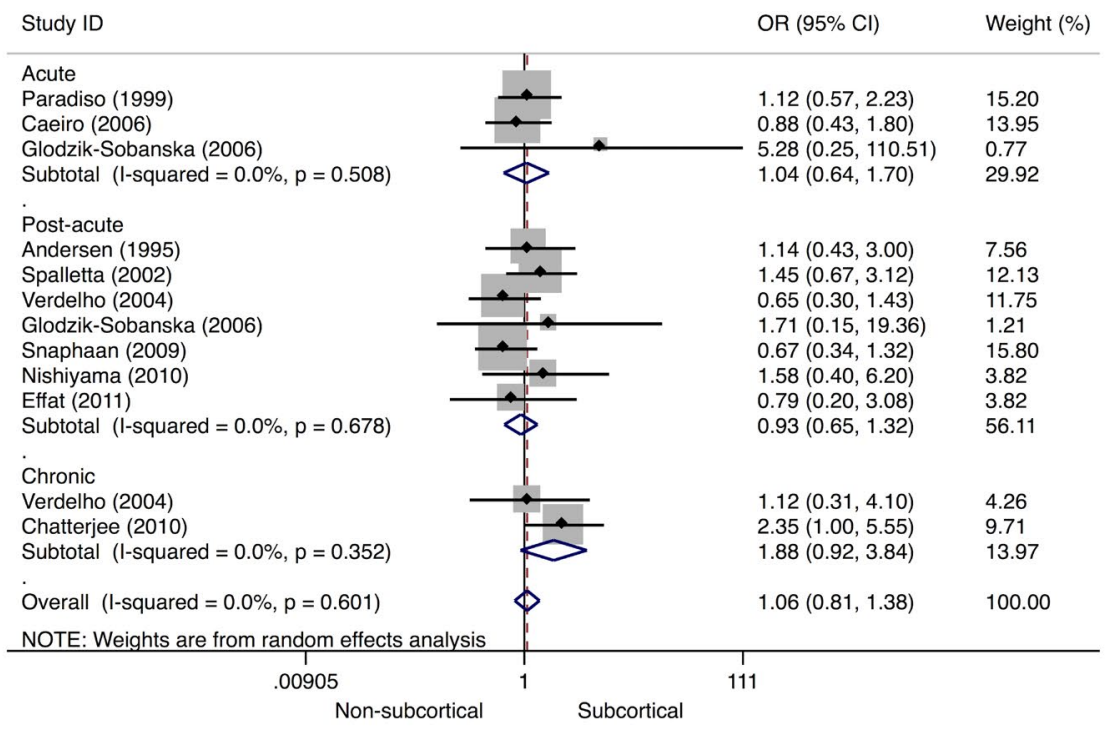

\section{B}

\begin{tabular}{|c|c|c|c|}
\hline Study ID & & OR $(95 \% \mathrm{Cl})$ & Weight $(\%)$ \\
\hline Acute & i & & \\
\hline Starkstein (1989) & $\frac{1}{1}$ & $0.68(0.14,3.19)$ & 4.36 \\
\hline Huwel (1998) & $\longrightarrow$ & $0.44(0.09,2.08)$ & 4.38 \\
\hline Wongwandee (2012) & $\frac{1}{1}$ & $6.00(1.09,33.02)$ & 3.82 \\
\hline Chen (2016) & + & $1.29(0.64,2.61)$ & 9.18 \\
\hline Metoki (2016) & $\stackrel{p}{a}$ & $2.13(0.97,4.66)$ & 8.58 \\
\hline Zhang (2016) & & $0.88(0.46,1.67)$ & 9.60 \\
\hline Subtotal $(\mathrm{I}$-squared $=41.4 \%, p=0.129$ ) & & $1.26(0.74,2.14)$ & 39.92 \\
\hline Post-acute & i & & \\
\hline Wichowicz (2006) & & $2.85(0.94,8.66)$ & 6.42 \\
\hline Nishiyama (2010) & $\frac{1}{1}$ & $2.47(0.99,6.15)$ & 7.67 \\
\hline Tang (2011) & 4 & $1.28(0.76,2.15)$ & 10.56 \\
\hline Jiang (2014) & $\longrightarrow$ & $7.10(3.68,13.70)$ & 9.52 \\
\hline Gu (2015) & -1 & $1.47(0.74,2.94)$ & 9.24 \\
\hline Wichowicz (2015) & $\frac{1}{1}$ & $2.99(1.22,7.33)$ & 7.77 \\
\hline Chen (2016) & 0 & $1.22(0.58,2.55)$ & 8.91 \\
\hline Subtotal $(I-$ squared $=71.2 \%, p=0.002)$ & & $2.25(1.33,3.84)$ & 60.08 \\
\hline Overall $(I-$ squared $=65.3 \%, p=0.001)$ & $\phi$ & $1.78(1.20,2.66)$ & 100.00 \\
\hline NOTE: Weights are from random effects analysis & & & \\
\hline \begin{tabular}{|c|c|c|} 
& 1 \\
.0303
\end{tabular} & & & \\
\hline
\end{tabular}

Figure 7. Forest plot of the relationship between post-stroke depression and subcortical/basal ganglia lesions

Note: In panel A, the results of the meta-analysis on subcortical lesion location are presented. Apart from the overall analysis, the subanalyses on acute stroke phase (upper panels), post-acute stroke phase (middle panels), and chronic stroke phase (lower panels) are presented. In panel B, the results of the meta-analysis on basal ganglia lesions are presented. Apart from the overall analysis, the subanalyses on acute stroke phase (upper panels) and post-acute stroke phase (lower panels) are presented. Abbreviations: $\mathrm{Cl}$, confidence interval; OR, odds ratio. 

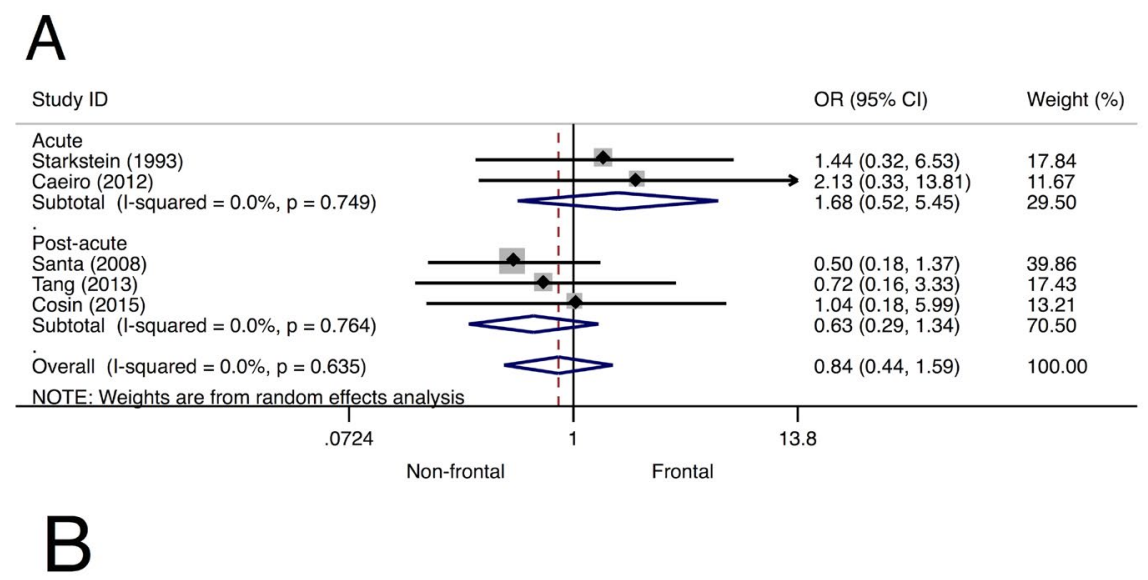

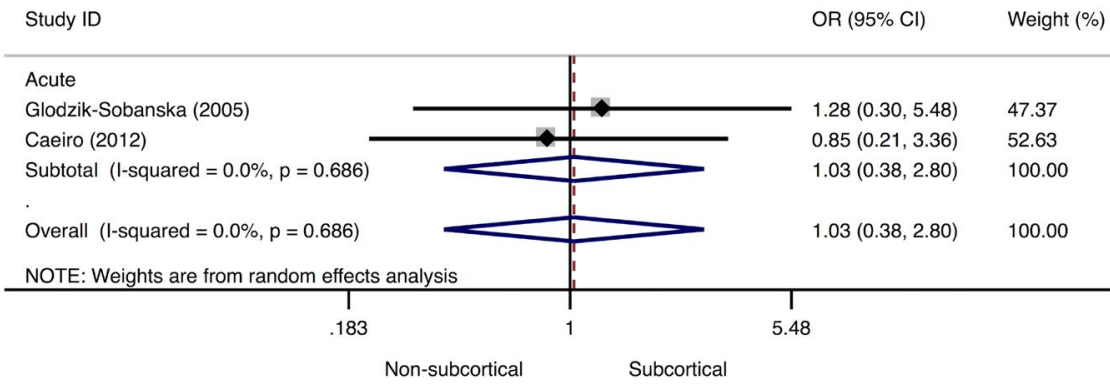

C

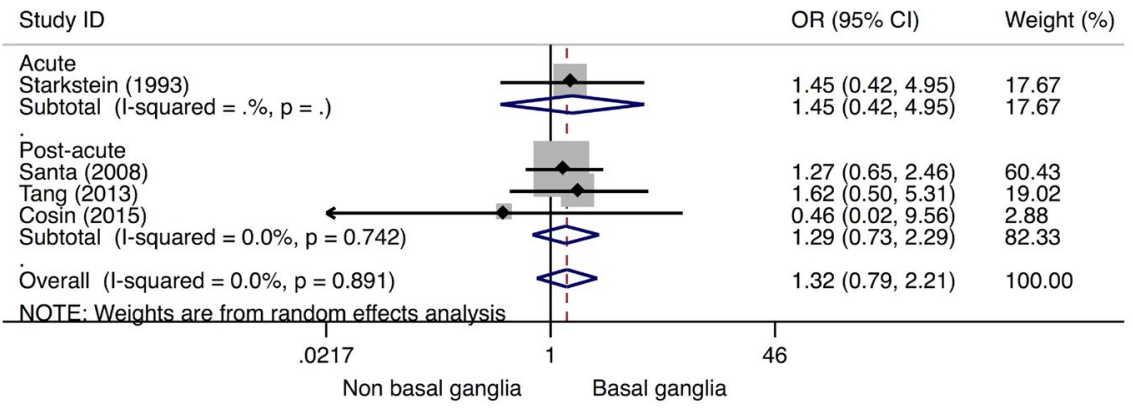

Figure 8. Forest plot of the relationship between post-stroke apathy and lesion location Note: In panel A, the results of the meta-analysis on frontal lesion location are presented. In panel B, the results of the meta-analysis on subcortical lesion location are presented. In panel $C$, the results of the meta-analysis on basal ganglia lesions are presented. Apart from the overall analysis, the subanalyses on acute stroke phase (upper panels) and post-acute stroke phase (lower panels) are presented. Abbreviations: $\mathrm{Cl}$, confidence interval; $\mathrm{OR}$, odds ratio. 


\section{Chapter 2}

Table 3. Imaging markers associated with post-stroke depression and post-stroke apathy

\begin{tabular}{|c|c|}
\hline \multirow[t]{2}{*}{ Imaging marker } & \multirow{2}{*}{$\begin{array}{l}\text { Acute phase } \\
\text { PSD }\end{array}$} \\
\hline & \\
\hline Degree of WMH & - \\
\hline Cerebral microbleeds & Choi-Kwon et al. $(2012)^{120}$ \\
\hline Large lesion volume & $\begin{array}{l}\text { Shimoda and Robinson (1999); }{ }^{68} \mathrm{Ku} \text { et al. }(2013) i^{125} \\
\text { Nys et al. (2005)126 }\end{array}$ \\
\hline Large number of lesions & - \\
\hline Metabolism & $\begin{array}{l}\text { Huang et al. }(2010)_{i}^{130} \\
\text { Xu et al. }(2008)^{131}\end{array}$ \\
\hline Atrophy & - \\
\hline Regional cerebral blood flow & - \\
\hline Functional connectivity / fractional anisotropy & $\begin{array}{l}\text { Altered FC in left orbital part of IFG: } \\
\text { Zhang et al. }(2014)^{138}\end{array}$ \\
\hline
\end{tabular}

\section{PSA}

Degree of WMH

Large lesion volume

Large number of lesions

Metabolism Glodzik-Sobanska et al. (2005) $\quad$ (141

Regional cerebral blood flow $\quad$ Bilateral BG: Onoda et al. (2011) 111

Fractional anisotropy

Atrophy

Abbreviations: ATR, atrophy; BG, basal ganglia; CC, corpus collosum; CMB, cerebral microbleeds; dIF, dorsolateral frontal; FA, fractional anisotropy; FC, functional connectivity; $F L$, frontal lobe; IFG, inferior frontal gyrus; IFT, left frontotemporal; $\mathrm{RH}$, right hemisphere; WM, white matter; WMC, white matter changes; WMH, white matter hyperintensities, PSA, post-stroke apathy; PSD, post-stroke depression. 


\section{Studies}

\section{Post-acute phase}

\section{Chronic phase}

Deep WMH: Kim et al. (2011); ${ }^{117}$ Tang et al. (2010)81

Overall, BG, frontal WMC: Chatterjee

Left frontal WMH: Mok et al. (2010) $)^{118}$ et al. (2010); ${ }^{89}$

Overall, deep, periventricular WMH:

Pavlovic et al. (2016) ${ }^{119}$

Tang et al. (2011a, 2011b, 2014a, 2014b)

Hama et al. (2007); ${ }^{9}$ MacHale et al. (1998); 106

Morris et al. (1992); ${ }^{72}$ Schwartz et al. (1993); ${ }^{87}$ Shimoda and

Robinson (1999); 68 Zhang et al. (2012) ${ }^{83}$

Sharpe et al. (1990, 1994); $i^{127,128}$

Shimoda and Robinson (1999) ${ }^{68}$

Bendsen et al. (1997); $i^{129}$ Jiang et al. (2014); ${ }^{98}$ Tang et al.

Chatterjee et al. (2010); $; 9$ Lacunar (2014): ${ }^{124}$ Zhang et al. (2012) $)^{83}$

lesions: Pavlovic et al. (2016) ${ }^{119}$

Glodzik-Sobanska et al. (2006); ${ }^{132}$ Wang et al. (2012): ${ }^{133}$

Xu et al. $(2008)^{131}$

Left IFG: Fu et al. (2010)134 FL: Tang et al. $(2013)^{135}$

Subcortical: Astrom et al. (1993); 66 Starkstein et al. (1988) ${ }^{136}$

Left hemisphere: Wichowicz et al. $(2006)^{137}$

Frontal WM integrity: Williamson et al. (2010). ${ }^{139}$

Increased ratio FA values in bilateral anterior limbs of IC:

Yasuno et al. (2014) $)^{140}$

$\mathrm{RH}$ WMH, right fronto-subcortical circuit WMH: Brodaty et al. $(2005) i^{13}$

Periventricular WMH: Tang et al. (2013) 115

Hama et al. (2007)

Tang et al. $(2013)^{115}$

Right dIF and IFT: Okada et al.

$(1997)^{142}$

Reduced FA in Genu of CC, left anterior corona radiata,

splenium of CC, and WM in the right IFG: Yang et al. (2015). ${ }^{143}$

Reduced median FA, reduction in WM integrity in anterior

cingulum, fornix and uncinate fasciculus: Hollocks et al.

$(2015)^{11}$

Frontal cortical atrophy: Mihalov et al. $(2016)^{112}$ 


\section{OTHER IMAGING MARKERS}

Several studies examined imaging markers other than lesion location and type in association with PSD. These markers could not be evaluated in a meta-analysis. Therefore, the most important imaging markers are described qualitatively (see Table 3). PSD was associated with total, ${ }^{89,119}$ deep, ${ }^{119}$ frontal, 89,118 and periventricular WMH. ${ }^{119}$ Also, cerebral microbleeds are associated with PSD, ${ }^{121-124}$ and several studies showed that PSD is more prevalent in patients with a large lesion volume, $9,68,72,83,87,106,125-128$ or large number of lesions. ${ }^{83,89,98,119,124,129}$

More recently, advanced diffusion tensor imaging (DTI) techniques have been used to investigate the association between microstructural abnormalities in white matter (WM) and PSD. Yasuno et al. ${ }^{140}$ showed that a reduction in fractional anisotropy (FA) in the bilateral anterior limbs of the internal capsule was associated with an increased risk of PSD and Williamson et al. ${ }^{139}$ showed that decreased WM integrity in the frontal lobes was associated with mood deficits. This indicates that WM damage in certain brain regions is associated with the development of PSD. A resting-state functional MRI (fMRI) study showed that altered functional connectivity in regions involved in affect was associated with higher levels of depression. ${ }^{138}$ Atrophy also seems to be an important predictor of PSD, as significant associations were found with frontal lobe atrophy, ${ }^{135}$ subcortical atrophy, ${ }^{66,136}$ and left inferior frontal gyrus atrophy. ${ }^{134}$ Interestingly, none of these studies reported on hippocampal atrophy. Recently, Chen et al. ${ }^{144}$ looked at medial temporal lobe atrophy, but found no association with PSD in the acute or post-acute stroke phase. According to proton magnetic resonance spectroscopy ( $\left.{ }^{1} \mathrm{H}-\mathrm{MRS}\right)$ studies, biochemical changes in metabolite levels in frontal lobe, ${ }^{131-133}$ hippocampus, ${ }^{130}$ and left thalamus ${ }^{130}$ seem to accompany the development of PSD.

Compared with PSD studies, only few studies evaluated imaging markers related to PSA (see Table 3). PSA was significantly associated with degree of righthemisphere, $^{13}$ right fronto-subcortical circuit, ${ }^{13}$ and periventricular $\mathrm{WMH}^{135}$ In addition, large lesion volume, ${ }^{9}$ and large number of lesions ${ }^{115}$ were associated with PSA. A recent study by Mihalov et al. ${ }^{112}$ showed that frontal cortical atrophy was a strong predictor of PSA, and this relation increased with higher age. In two DTI studies reductions in FA in several brain areas were associated with an increased level of apathy. ${ }^{143,145}$ In addition, PSA was associated with reductions in regional cerebral blood flow in the bilateral basal ganglia, ${ }^{111}$ right dorsolateral frontal cortex, and left frontotemporal cortex ${ }^{142}$ measured with single-photon emission computed tomography. An $\mathrm{H}^{1}$-MRS study suggested that lower $\mathrm{N}$-acetylaspartate/ creatine ratio in the right frontal lobe was related to PSA. ${ }^{141}$ 


\section{META-REGRESSION ANALYSES}

Egger's regression tests showed no evidence for statistically significant smallstudy effects in above meta-analyses (see Table 1), although it was not possible to calculate Egger's regression coefficients for the association with subcortical lesions in PSA as the pooled sample size was too small. Visual inspection of the shape of the funnel plots also did not reveal convincing evidence of obvious asymmetry (see supplementary Online Resource 6). However, some plots, especially for the PSA studies, only consisted of few studies.

Meta-regression analyses were performed to assess potential sources of heterogeneity between PSD studies reporting on lesion laterality $(n=60)$. None of the included variables appeared to be a significant cause of heterogeneity. In addition, meta-regression analyses were performed on PSD studies reporting on frontal $(n=30)$ and basal ganglia lesions $(n=12)$. Only study phase appeared to be a significant cause of heterogeneity in both analyses (frontal: $p=0.041$, residual $R^{2}=58.9 \%$, Adj. $R^{2}=25.7 \%$; basal ganglia: $p=0.044$, residual $R^{2}=55.4 \%$, Adj. $R^{2}=$ $50.7 \%)$. To assess potential sources of heterogeneity among PSA studies reporting on lesion laterality $(n=9)$, meta-regression analyses were performed showing that only imaging method appeared to be an important cause of heterogeneity ( $p=$ 0.052 , residual $I^{2}=31.2 \%$, Adj. $R^{2}=64.6 \%$ ).

Table 4. Circuits associated with post-stroke depression and post-stroke apathy

\begin{tabular}{|lcl|}
\hline Studies & Phase & \multicolumn{1}{c|}{ Circuits - network } \\
\hline Terroni et al. $(2011)^{146}$ & Acute & $\begin{array}{l}\text { Disruption of limbic-cortical-striatal-pallidal-thalamic circuit, } \\
\text { Medial PFC dysfunction }\end{array}$ \\
\hdashline Yang et al. $(2015)^{90}$ & Acute & $\begin{array}{l}\text { Frontal lobe, insula, limbic system, parietal lobe, basal ganglia, } \\
\text { temporal lobe }\end{array}$ \\
\hdashline Vataja et al. $(2001)^{147}$ & Post-acute & $\begin{array}{l}\text { Higher number and lesion volume in (left) prefronto-subcortical } \\
\text { circuit }\end{array}$ \\
\hdashline Tang et al. $(2011)^{148}$ & Post-acute & \begin{tabular}{l} 
Lesions in frontal subcortical circuits \\
\hline Yang et al. $(2015)^{145}$
\end{tabular} \\
\hline
\end{tabular}

Abbreviations: PFC, prefrontal cortex; PSA, post-stroke apathy; PSD, post-stroke depression. 


\section{DISCUSSION}

This systematic review and meta-analysis summarizes the most up-to-date information on a range of imaging markers associated with PSD and PSA during the acute, post-acute, and chronic stroke phase. Meta-analyses indicated that PSD in the post-acute phase was significantly more frequent in patients with frontal or basal ganglia lesions. No significant association was found between PSD and lesion laterality in the post-acute and chronic stroke phase. Nevertheless, it is of interest to mention that left-sided stroke occurred more often in the PSD group in the acute phase. This result became insignificant after the inclusion of four recent large studies, $73,144,149,150$ which differed from the other studies in that they reported a relatively low PSD prevalence (median 18.6\%, IQR 17.4-30.2). Frequency of PSD was equal for ischemic and hemorrhagic stroke in all stroke phases, but PSA was more frequent after hemorrhagic stroke in the acute phase, whereas it was more frequent after ischemic stroke in the post-acute phase. Since only four PSA studies were available, this finding should be interpreted with caution. Also, PSA did not depend on lesion laterality or location, but again the amount of available PSA studies was small in general.

Our meta-analysis updates and extends previous studies. The meta-analysis by Wei et al..$^{23}$ on lesion laterality and PSD found a significant association between right hemispheric lesions and risk of PSD in the post-acute stroke phase (1-6 months). In contrast to Wei et al., ${ }^{23}$ we defined the post-acute period as 15 days to 6 months, which could explain the difference in results. In agreement with Caeiro et al. ${ }^{26}$ the prevalence of PSA was not associated with lesion laterality. Both metaanalyses did not study associations with markers other than lesion laterality and lesion type, while the review of van Dalen et al. ${ }^{6}$ evaluated associations between PSA and lesion location only qualitatively and concluded that no clear association could be found.

The present findings suggest that lesion location is an important risk factor for PSD in the post-acute stroke phase. However, in the past few years the hypothesis of PSD and PSA being associated with damage to specific lesion locations has been shifted to the idea that damage to a neuronal network involved in affect is underlying the development of PSD and PSA, ${ }^{146-148}$ with different sub-circuits involved in $\mathrm{PSD}^{90}$ and $\mathrm{PSA},{ }^{145}$ see Table 4 . DTI is a promising tool to identify more accurately how these brain networks are affected after stroke. The qualitative overview of imaging markers associated with PSD and PSA showed that not only direct stroke-related features, such as lesion location, lesion volume, and number of lesions, but also other neurovascular, non-directly stroke-related but often co-occurring features, such as degree of $\mathrm{WMH}$, cerebral microbleeds, and 
atrophy, were frequently associated with PSD. With respect to PSA, associations with degree of $\mathrm{WMH}$, lesion volume, and number of lesions were found in some extent. Co-occurring vascular lesions may make a stroke patient more vulnerable for developing PSD and PSA. Therefore, future studies should focus on a broader range of imaging markers, including lesion volume, atrophy, $\mathrm{WMH}$, and cerebral microbleeds, and also how lesion-related markers may interact with co-occuring indirect vascular markers. Besides, advanced imaging techniques (e.g. DTI, fMRI) are needed to evaluate how microstructural abnormalities and changes in functional connectivity contribute to the development of PSD and PSA.

Our study has the following strengths. A large amount of publications on PSD were identified, resulting in a rich pooled cohort of studies that were not included in earlier meta-analyses. ${ }^{73,82,84,95,98,144,149-152}$ Furthermore, beside information on lesion laterality, also data on other imaging markers was retrieved for quantitative and qualitative analysis. Therefore, the present review provides an up-to-date and extended overview of findings on the association between imaging markers and risk of PSD and PSA.

One limitation of the present study was the small amount of studies on PSA, which made it difficult to perform subanalyses. Therefore, future studies are needed on imaging markers of PSA, covering a broad range of imaging markers. Nevertheless, as heterogeneity was small between PSA studies, we believe that the results are still of importance, but should be interpreted with caution as the generalizability and validity is compromised in comparison with meta-analyses including a larger amount of studies. In addition, moderate to high (unexplained) heterogeneity between studies in some meta-analyses indicated large differences in methodology between studies. Particularly the use of different scales and cut-offs to define the presence of depression and apathy and different imaging methods (CT vs. MRI) are of influence on the comparability of findings. Also, differences in eligibility criteria (e.g. exclusion of patients with aphasia, differences in age range) can create heterogeneity among studies. Meta-regression analyses were performed to identify potential sources of heterogeneity, and only study phase for PSD studies and imaging method for PSA studies could be identified. However, in addition to the included variables, also other potential variables (e.g. years of education, cognitive status), that could not be included in the analyses due to the large variability in the methods and availability of data between studies, might explain some of the between-study difference in effect estimates. Therefore, we performed random-effects meta-analyses, which take the heterogeneity between studies into account. 


\section{Chapter 2}

\section{CONCLUSION}

The present study suggests that lesion location rather than lesion laterality or type may be an important risk factor for PSD in the post-acute stroke phase. In contrast, lesion type rather than lesion laterality or location might be an important factor in determining who is at risk to develop PSA in the acute and post-acute phase, though additional studies are needed to confirm this, as the sample size was small. Therefore, large multicenter cohort studies using advanced imaging techniques and focusing on both PSD and PSA from the acute to the chronic stroke phase are strongly needed. 


\section{REFERENCES}

1. Hackett ML, Köhler S, O'Brien JT, Mead GE. Neuropsychiatric outcomes of stroke. Lancet Neurol. 2014;13:525-534.

2. Hackett ML, Yapa C, Parag V, Anderson CS. Frequency of depression after stroke a systematic review of observational studies. Stroke. 2005;36:1330-1340.

3. Marin RS. Differential diagnosis and classification of apathy. Am J Psychiatry. 1990;147:22-30.

4. Levy ML, Cummings JL, Fairbanks LA, Masterman D, Miller BL, Craig AH, et al. Apathy is not depression. J Neuropsychiatry Clin Neurosci. 1998;10:314-319.

5. Marin RS, Firinciogullari S, Biedrzycki RC. The sources of convergence between measures of apathy and depression. J Affect Disord. 1993;28:117-124.

6. van Dalen JW, van Charante EPM, Nederkoorn PJ, van Gool WA, Richard E. Poststroke apathy. Stroke. 2013;44:851-860.

7. Hama S, Yamashita H, Yamawaki S, Kurisu K. Post-stroke depression and apathy: Interactions between functional recovery, lesion location, and emotional response. Psychogeriatrics. 2011;11:68-76.

8. Andersson S, Krogstad J, Finset A. Apathy and depressed mood in acquired brain damage: Relationship to lesion localization and psychophysiological reactivity. Psychol Med. 1999;29:447456.

9. Hama S, Yamashita H, Shigenobu M, Watanabe A, Kurisu K, Yamawaki S, et al. Post-stroke affective or apathetic depression and lesion location: Left frontal lobe and bilateral basal ganglia. Eur Arch Psychiatry Clin Neurosci. 2007;257:149-152.

10. Hama S, Murakami T, Yamashita H, Onoda K, Yamawaki S, Kurisu K. Neuroanatomic pathways associated with monoaminergic dysregulation after stroke. Int J Geriatr Psychiatry. 2017;32:633642.

11. Hollocks M, Lawrence A, Brookes R, Barrick T, Morris R, Husain M, et al. Differential relationships between apathy and depression with white matter microstructural changes and functional outcomes. Brain. 2015;138:3803-3815.

12. Murakami T, Hama S, Yamashita H, Onoda K, Kobayashi M, Kanazawa J, et al. Neuroanatomic pathways associated with poststroke affective and apathetic depression. Am J Geriatr Psychiatry. 2013;21:840-847.

13. Brodaty H, Sachdev PS, Withall A, Altendorf A, Valenzuela MJ, Lorentz L. Frequency and clinical, neuropsychological and neuroimaging correlates of apathy following stroke--the sydney stroke study. Psychol Med. 2005;35:1707-1716.

14. Withall A, Brodaty $H$, Altendorf A, Sachdev PS. A longitudinal study examining the independence of apathy and depression after stroke: The sydney stroke study. Int Psychogeriatr. 2011;23:264273.

15. Caeiro L, Ferro JM, Pinho e Melo T, Canhão P, Figueira ML. Post-stroke apathy: An exploratory longitudinal study. Cerebrovas Dis. 2013;35:507-513.

16. Hama S, Yamashita H, Shigenobu M, Watanabe A, Hiramoto K, Kurisu K, et al. Depression or apathy and functional recovery after stroke. Int J Geriatr Psychiatry. 2007;22:1046-1051.

17. Pohjasvaara T, Vataja R, Leppävuori $A$, Kaste M, Erkinjuntti T. Depression is an independent predictor of poor long-term functional outcome post-stroke. Eur J Neurology. 2001;8:315-319.

18. Carod-Artal J, Egido JA, González JL, De Seijas EV. Quality of life among stroke survivors evaluated 1 year after stroke experience of a stroke unit. Stroke. 2000;31:2995-3000.

19. Mayo NE, Fellows LK, Scott SC, Cameron J, Wood-Dauphinee S. A longitudinal view of apathy and its impact after stroke. Stroke. 2009;40:3299-3307.

20. Ramasubbu R, Kennedy SH. Factors complicating the diagnosis of depression in cerebrovascular disease, Part I. Phenomenological and nosological issues. Can J Psychiatry. 1994;39:496-600. 
21. Carson AJ, MacHale S, Allen K, Lawrie SM, Dennis M, House A, et al. Depression after stroke and lesion location: A systematic review. Lancet. 2000;356:122-126.

22. Kutlubaev MA, Hackett ML. Part ii: Predictors of depression after stroke and impact of depression on stroke outcome: An updated systematic review of observational studies. Int J Stroke. 2014;9:1026-1036.

23. Wei N, Yong W, Li X, Zhou Y, Deng M, Zhu H, et al. Post-stroke depression and lesion location: A systematic review. J Neurol. 2015;262:81-90.

24. Caeiro L, Ferro JM, Figueira ML. Apathy in acute stroke patients. Eur J Neurology. 2012;19:291297.

25. Starkstein SE, Fedoroff JP, Price TR, Leiguarda R, Robinson RG. Apathy following cerebrovascular lesions. Stroke. 1993;24:1625-1630.

26. Caeiro L, Ferro JM, Costa J. Apathy secondary to stroke: A systematic review and meta-analysis. Cerebrovasc Dis. 2013;35:23-39.

27. Liberati A, Altman DG, Tetzlaff J, Mulrow C, Gøtzsche PC, loannidis JP, et al. The prisma statement for reporting systematic reviews and meta-analyses of studies that evaluate health care interventions: Explanation and elaboration. Ann Intern Med. 2009;151: 65-94.

28. DerSimonian R, Laird N. Meta-analysis in clinical trials. Control Clin Trials. 1986;7:177-188.

29. Buisman LR, Tan SS, Nederkoorn PJ, Koudstaal PJ, Redekop WK. Hospital costs of ischemic stroke and tia in the netherlands. Neurology. 2015;84:2208-2215.

30. Prasad K, Kaul S, Padma M, Gorthi S, Khurana D, Bakshi A. Stroke management. Ann Indian Acad Neurol. 2011;14:82-96.

31. Aziz NA. Long-term rehabilitation after stroke: Where do we go from here? Rev Clin Gerontol. 2010;20:239-245.

32. Wells G, Shea B, O'connell D, Peterson J, Welch V, Losos M, et al. The newcastle-ottawa scale (nos) for assessing the quality of nonrandomised studies in meta-analyses. 2000. Available from: http:// www.ohri.ca/programs/clinical_epidemiology/oxford.asp.

33. Egger M, Smith GD, Schneider M, Minder C. Bias in meta-analysis detected by a simple, graphical test. BMJ. 1997;315:629-634.

34. Akiashvili N, Beridze M, Janelidze M, Lobjanidze N, Kvirkvelia N. Cognitive outcome in multiple silent lacunar lesions and stroke in geriartric patients. Cerebrovasc Dis. 2013;36:76.

35. Toso V, Gandolfo C, Paolucci S, Provinciali L, Torta R, Grassivaro N, et al. Post-stroke depression: Research methodology of a large multicentre observational study (destro). Neurolo Sci. 2004; $25: 138-144$.

36. Beckson M, Cummings JL. Neuropsychiatric aspects of stroke. Int J Psychiatry Med. 1991;21:1-15.

37. Robinson RG, Starkstein SE. Mood disorders following stroke: New findings and future directions. J Geriatr Psychiatry. 1989;22:1-15.

38. Beblo T, Wallesch C-W, Herrmann M. The crucial role of frontostriatal circuits for depressive disorders in the postacute stage after stroke. Neuropsychiatry Neuropsychol Behav Neurol. 1999; 12:236-246.

39. Grasso MG, Pantano P, Ricci M, Intiso DF, Pace A, Padovani A, et al. Mesial temporal cortex hypoperfusion is associated with depression in subcortical stroke. Stroke. 1994;25:980-985.

40. Lassalle-Lagadec S, Catheline G, Mayo W, Dilharreguy B, Renou P, Fleury O, et al. Cerebellum involvement in post-stroke mood: A combined ecological and mri study. Psychiatry Res. 2013;212:158-160.

41. Lassalle-Lagadec S, Sibon I, Dilharreguy B, Renou P, Fleury O, Allard M. Subacute default mode network dysfunction in the prediction of post-stroke depression severity. Radiology. 2012;264:218-224.

42. Matsuoka K, Yasuno F, Taguchi A, Yamamoto A, Kajimoto K, Kazui H, et al. Delayed atrophy in posterior cingulate cortex and apathy after stroke. Int J Geriatr Psychiatry. 2015;30:566-572. 
43. Mayberg HS, Robinson RG, Wong DF, Parikh R, Bolduc P, Starkstein SE, et al. PET imaging of cortical s2 serotonin receptors after stroke: Lateralized changes and relationship to depression. Am J Psychiatry. 1988;145:937-943.

44. Paradiso S, Ostedgaard K, Vaidya J, Ponto LB, Robinson R. Emotional blunting following left basal ganglia stroke: The role of depression and fronto-limbic functional alterations. Psychiatry Res. 2013;211:148-159.

45. Ramasubbu R, Flint A, Brown G, Awad G, Kennedy S. A neuroendocrine study of serotonin function in depressed stroke patients compared to non depressed stroke patients and healthy controls. J Affect Disord. 1999;52:121-133.

46. Astrom M. Generalized anxiety disorder in stroke patients. A 3-year longitudinal study. Stroke. 1996;27:270-275.

47. Downhill JE, Jr., Robinson RG. Longitudinal assessment of depression and cognitive impairment following stroke. J Nerv Ment Dis. 1994;182:425-431.

48. Vataja R, Pohjasvaara T, Mäntylä R, Ylikoski R, Kalska H, Leskelä M, et al. Depression-executive dysfunction syndrome in stroke patients. Am J Geriatr Psychiatry. 2005;13:99-107.

49. Bella R, Pennisi G, Cantone M, Palermo F, Pennisi M, Lanza G, et al. Clinical presentation and outcome of geriatric depression in subcortical ischemic vascular disease. Gerontology. 2010;56:298-302.

50. Grool AM, Gerritsen L, Zuithoff NP, Mali WP, van der Graaf Y, Geerlings MI. Lacunar infarcts in deep white matter are associated with higher and more fluctuating depressive symptoms during three years follow-up. Biol Psychiatry. 2013;73:169-176.

51. O'Brien JT, Firbank MJ, Krishnan MS, van Straaten ECW, van der Flier WM, Petrovic K, et al. White matter hyperintensities rather than lacunar infarcts are associated with depressive symptoms in older people: The ladis study. Am J Geriatric Psychiatry. 2006;14:834-841.

52. Ojagbemi A, Owolabi M, Atalabi M, Baiyewu O. Stroke lesions and post-stroke depression among survivors in ibadan, nigeria. Afr J Med Med Sci. 2013;42:245-251.

53. Sachdev PS, Chen X, Joscelyne A, Wen W, Brodaty H. Amygdala in stroke/transient ischemic attack patients and its relationship to cognitive impairment and psychopathology: The Sydney stroke study. Am J Geriatr Psychiatry. 2007;15:487-496.

54. Tanislav C, Kropp P, Grittner U, Holzhausen M, Fazekas F, Jungehülsing GJ, et al. Clinically relevant depressive symptoms in young stroke patients-results of the sifap1 study. Neuroepidemiology. 2015:44:30-38.

55. Wu R-H, Li Q, Tan Y, Liu X-Y, Huang J. Depression in silent lacunar infarction: A cross-sectional study of its association with location of silent lacunar infarction and vascular risk factors. Neurol Sci. 2014;35:1553-1559.

56. Eriksen S, Gay CL, Lerdal A. Acute phase factors associated with the course of depression during the first 18 months after first-ever stroke. Disabil Rehabil. 2016;38:30-35.

57. Sheehan DV, Lecrubier Y, Sheehan KH, Amorim P, Janavs J, Weiller E, et al. The mini-international neuropsychiatric interview (MINI): The development and validation of a structured diagnostic psychiatric interview for DSM-IV and ICD-10. J Clin Psychiatry. 1998;59:22-33.

58. Spitzer R, Gibbon M, Williams J. Structured clinical interview for axis I DSM-IV disorders (SCID). Washington, DC: American Psychiatric Association. 1995.

59. American Psychiatric Association. DSM-III: Diagnostic and statistical manual of psychiatric disorders. APA, Washington. 1980.

60. American Psychiatric Association. Diagnostic and statistical manual (DSM-IV). Washington, DC: American Psychiatric Association. 1994.

61. Hamilton M. A rating scale for depression. J Neurol Neurosurg Psychiatry. 1960;23:56-62.

62. Montgomery SA, Asberg M. A new depression scale designed to be sensitive to change. $\mathrm{Br} \mathrm{J}$ Psychiatry. 1979;134:382-389. 
63. Yesavage JA, Brink TL, Rose TL, Lum O, Huang V, Adey M, et al. Development and validation of a geriatric depression screening scale: A preliminary report. J Psychiatric Res. 1983;17:37-49.

64. Starkstein SE, Mayberg HS, Preziosi T, Andrezejewski P, Leiguarda R, Robinson R. Reliability, validity, and clinical correlates of apathy in parkinson's disease. J Neuropsychiatry Clin Neurosci. 1992;4:134-139.

65. Marin RS, Biedrzycki RC, Firinciogullari S. Reliability and validity of the apathy evaluation scale. Psychiatry Res. 1991;38:143-162.

66. Astrom M, Adolfsson R, Asplund K. Major depression in stroke patients. A 3-year longitudinal study. Stroke. 1993;24:976-982.

67. Herrmann M, Bartels C, Wallesch C-W. Depression in acute and chronic aphasia: Symptoms, pathoanatomical-clinical correlations and functional implications. I Neurol Neurosurg Psychiatry.1993;56:672-678.

68. Shimoda K, Robinson RG. The relationship between poststroke depression and lesion location in long-term follow-up. Biol Psychiatry. 1999;45:187-192.

69. Dam H, Pedersen HE, Ahlgren P. Depression among patients with stroke. Acta Psychiatr Scand. 1989;80:118-124.

70. House A, Dennis M, Warlow C, Hawton K, Molyneux A. Mood disorders after stroke and their relation to lesion location. A ct scan study. Brain. 1990;113:1113-1129.

71. Kim JS, Choi-Kwon S. Poststroke depression and emotional incontinence: Correlation with lesion location. Neurology. 2000;54:1805-1810.

72. Morris PL, Robinson RG, Raphael B. Lesion location and depression in hospitalized stroke patients: Evidence supporting a specific relationship in the left hemisphere. Neuropsychiatry, Neuropsychol Behav Neurol. 1992;5:75-82.

73. Metoki N, Sugawara N, Hagii J, Saito S, Shiroto H, Tomita T, et al. Relationship between the lesion location of acute ischemic stroke and early depressive symptoms in Japanese patients. Ann Gen Psychiatry. 2016;15:1-6.

74. Robinson RG, Kubos KL, Starr LB, Rao K, Price TR. Mood disorders in stroke patients. Importance of location of lesion. Brain. 1984;107:81-93.

75. Shi YZ, Xiang YT, Wu SL, Zhang N, Zhou J, Bai Y, et al. The relationship between frontal lobe lesions, course of post-stroke depression, and 1-year prognosis in patients with first-ever ischemic stroke. PLOS ONE. 2014;9:e100456.

76. Aben I, Ladder J, Honig A, Lousberg R, Boreas A, Verhey FRJ. Focal or generalized vascular brain damage and vulnerability to depression after stroke: A 1-year prospective follow-up study. Int Psychogeriat. 2006;18:19-35.

77. Effat SM, Mohamed MM, El Essawy HI, El Sheikh MM, Abdul Aal HS. Predictors and consequences of post-stroke depression in a sample of egyptian patients. Arab J Psychiatry. 2011;22:19-26.

78. Morris PLP, Robinson RG, Raphael B, Hopwood MJ. Lesion location and poststroke depression. J Neuropsychiatry Clin Neurosci. 1996;8:399-403.

79. Singh A, Black SE, Herrmann N, Leibovitch FS, Ebert PL, Lawrence J, et al. Functional and neuroanatomic correlations in poststroke depression: The sunnybrook stroke study. Stroke. 2000;31:637-644.

80. Stojanovic Z, Stojanovic SV. Emotional reactions in patients after frontal lobe stroke. Vojnosanitetski Pregled. 2015;72:770-778.

81. Tang WK, Chen YK, Lu JY, Chu WC, Mok VC, Ungvari GS, et al. White matter hyperintensities in post-stroke depression: A case control study. J Neurol Neurosurg Psychiatry. 2010;81:1312-1315.

82. Wichowicz HM, Gąsecki D, Lass P, Landowski J, Świerkocka M, Wiśniewski G, et al. Clinical utility of chosen factors in predicting post-stroke depression: A one year follow-up. Psychiatr. Pol. 2015;49:683-696.

83. Zhang $T$, Jing $X$, Zhao $X$, Wang $C$, Liu Z, Zhou Y, et al. A prospective cohort study of lesion location and its relation to post-stroke depression among chinese patients. J Affect Disord. 2012;136:8387. 
84. Terroni L, Amaro E, losifescu DV, Mattos P, Yamamoto FI, Tinone G, et al. The association of poststroke anhedonia with salivary cortisol levels and stroke lesion in hippocampal/parahippocampal region. Neuropsychiatr Dis Treat. 2015;11:233-242.

85. Paradiso S, Robinson RG. Minor depression after stroke: An initial validation of the dsm-iv construct. Am J Geriatr Psychiatry. 1999;7:244-251.

86. Starkstein SE, Robinson RG, Honig MA, Parikh RM, Joselyn J, Price TR. Mood changes after righthemisphere lesions. Br J Psychiatry. 1989;155:79-85.

87. Schwartz JA, Speed NM, Brunberg JA, Brewer TL, Brown M, Greden JF. Depression in stroke rehabilitation. Biol Psychiatry. 1993;33:694-699.

88. Tang WK, Chan SSM, Chiu HFK, Ungvari GS, Wong KS, Kwok TCY, et al. Poststroke depression in chinese patients: Frequency, psychosocial, clinical, and radiological determinants. J Geriatr Psychiatry Neurol. 2005;18:45-51.

89. Chatterjee K, Fall S, Barer D. Mood after stroke: A case control study of biochemical, neuroimaging and socio-economic risk factors for major depression in stroke survivors. BMC Neurol. 2010;10:1-10.

90. Yang S, Hua P, Shang X, Cui Z, Zhong S, Gong G, et al. A significant risk factor for poststroke depression: The depression-related subnetwork. J Psychiatry Neurosci. 2015;40:259-268.

91. Herrmann M, Bartels C, Schumacher M, Wallesch CW. Poststroke depression. Is there a pathoanatomic correlate for depression in the postacute stage of stroke? Stroke. 1995;26:850856.

92. Nishiyama Y, Komaba Y, Ueda M, Nagayama H, Amemiya S, Katayama Y. Early depressive symptoms after ischemic stroke are associated with a left lenticulocapsular area lesion. J Stroke Cerebrovasc Dis. 2010;19:184-189.

93. Robinson RG, Lipsey JR, Bolla-Wilson K, Bolduc PL, Pearlson GD, Rao K, et al. Mood disorders in left-handed stroke patients. Am J Psychiatry. 1985;142:1424-1429.

94. Morris PL, Robinson RG, de Carvalho ML, Albert P, Wells JC, Samuels JF, et al. Lesion characteristics and depressed mood in the stroke data bank study. J Neuropsychiatry Clin Neurosci. 1996;8:153159.

95. Saxena A, Suman A. Magnitude and determinants of depression in acute stroke patients admitted in a rural tertiary care hospital. J Neurosci Rural Pract. 2015;6:202-207.

96. Wongwandee M, Tangwongchai S, Phanthumchinda K. Relationship between poststroke depression and ischemic lesion location. J Med Assoc Thai. 2012;95:330-336.

97. Barker-Collo SL. Depression and anxiety 3 months post stroke: Prevalence and correlates. Arch Clin Neuropsychology. 2007;22:519-531.

98. Jiang XG, Lin Y, Li YS. Correlative study on risk factors of depression among acute stroke patients. Eur Rev Med Pharmacol Sci. 2014;18:1315-1323.

99. Rajashekaran P, Pai K, Thunga R, Unnikrishnan B. Post-stroke depression and lesion location: A hospital based cross-sectional study. Indian J Psychiatry. 2013;55:343-348.

100. Parikh RM, Lipsey JR, Robinson RG, Price TR. A two year longitudinal study of poststroke mood disorders: Prognostic factors related to one and two year outcome. Int J Psychiatry Med. 1988; 18:45-56.

101. Provinciali L, Paolucci S, Torta R, Toso V, Gobbi B, Gandolfo C. Depression after first-ever ischemic stroke: The prognostic role of neuroanatomic subtypes in clinical practice. Cerebrovasc Dis. 2008;26:592-599.

102. Rashid N, Clarke C, Rogish M. Post-stroke depression and expressed emotion. Brain Inj. 2013;27:223-238.

103. Stern RA, Bachman DL. Depressive symptoms following stroke. Am J Psychiatry. 1991;148:351356.

104. Andersen G, Vestergaard K, Ingemann-Nielsen M, Lauritzen L. Risk factors for post-stroke depression. Acta Psychiatr Scand. 1995;92:193-198. 
105. Castellanos-Pinedo F, Hernandez-Perez JM, Zurdo M, Rodriguez-Funez B, Hernandez-Bayo JM, Garcia-Fernandez C, et al. Influence of premorbid psychopathology and lesion location on affective and behavioral disorders after ischemic stroke. J Neuropsychiatry Clin Neurosci. 2011;23:340-347.

106. MacHale SM, O'Rourke SJ, Wardlaw JM, Dennis MS. Depression and its relation to lesion location after stroke. J Neurol Neurosurg Psychiatry. 1998;64:371-374.

107. Oladiji JO, Akinbo SRA, Aina OF, Aiyejusunle CB. Risk factors of post-stroke depression among stroke survivors in Lagos, Nigeria. Afr J Psychiatry (South Africa). 2009;12:47-51.

108. Verdelho A, Henon $H$, Lebert F, Pasquier F, Leys D. Depressive symptoms after stroke and relationship with dementia: A three-year follow-up study. Neurology. 2004;62:905-911.

109. Iranmanesh F, Vakilian A. Post stroke depression among Iranian patients. Neurosciences. 2009; 14:148-151

110. Desmond DW, Remien RH, Moroney JT, Stern Y, Sano M, Williams JB. Ischemic stroke and depression. J Int Neuropsychol Soc. 2003;9:429-439.

111. Onoda K, Kuroda Y, Yamamoto Y, Abe S, Oguro H, Nagai A, et al. Post-stroke apathy and hypoperfusion in basal ganglia: Spect study. Cerebrovasc Dis. 2011;31:6-11.

112. Mihalov J, Mikula P, Budiš J, Valkovič P. Frontal cortical atrophy as a predictor of poststroke apathy. J Geriatr Psychiatry Neurol. 2016;29:171-176.

113. Santa N, Sugimori H, Kusuda K, Yamashita Y, Ibayashi S, lida M. Apathy and functional recovery following first-ever stroke. Int J Rehabil Res. 2008;31:321-326.

114. Rochat L, Van der Linden M, Renaud O, Epiney J-B, Michel P, Sztajzel R, et al. Poor reward sensitivity and apathy after stroke implication of basal ganglia. Neurology. 2013;81:1674-1680.

115. Tang WK, Chen YK, Liang HJ, Chu WC, Mok VC, Ungvari GS, et al. Location of infarcts and apathy in ischemic stroke. Cerebrovasc Dis. 2013;35:566-571.

116. Kang SY, Kim JS. Anterior cerebral artery infarction stroke mechanism and clinical-imaging study in 100 patients. Neurology. 2008;70:2386-2393.

117. Kim JT, Park MS, Yoon GJ, Jung HJ, Choi KH, Nam TS, et al. White matter hyperintensity as a factor associated with delayed mood disorders in patients with acute ischemic stroke. Eur Neurol. 2011;66:343-349.

118. Mok VC, Wong A, Wong K, Chu WC, Xiong Y, Chan AY, et al. Executive dysfunction and left frontal white matter hyperintensities are correlated with neuropsychiatric symptoms in stroke patients with confluent white matter hyperintensities. Dement Geriatr Cogn Disord. 2010;30:254-260.

119. Pavlovic AM, Pekmezovic T, Zidverc Trajkovic J, Svabic Medjedovic T, Veselinovic N, Radojicic A, et al. Baseline characteristic of patients presenting with lacunar stroke and cerebral small vessel disease may predict future development of depression. Int J Geriatr Psychiatry. 2016;31:58-65.

120. Choi-Kwon S, Han K, Choi S, Suh M, Kim YJ, Song H, et al. Poststroke depression and emotional incontinence: Factors related to acute and subacute stages. Neurology. 2012;78:1130-1137.

121. Tang WK, Chen YK, Lu JY, Chu WC, Mok VC, Ungvari GS, et al. Cerebral microbleeds and depression in lacunar stroke. Stroke. 2011;42:2443-2446.

122. Tang WK, Chen YK, Lu JY, Chu WC, Mok VC, Ungvari GS, et al. Cerebral microbleeds and symptom severity of post-stroke depression: A magnetic resonance imaging study. J Affect Disord. 2011;129:354-358.

123. Tang WK, Chen Y, Liang H, Chu WC, Mok VC, Ungvari GS, et al. Cerebral microbleeds as a predictor of 1-year outcome of poststroke depression. Stroke. 2014;45:77-81.

124. Tang WK, Liu XX, Chen YK, Abrigo J, Chu WCW, Mok VCT, et al. Pontine microbleeds and depression in stroke. J Geriatr Psychiatry and Neurology. 2014;27:159-164.

125. Ku H-L, Chen C-H, Yang Y-T, Hu C-J, Wu D, Chen C-C, et al. Association between cerebral lesions and emotional changes in acute ischemic stroke patients. J Nerv Ment Dis. 2013;201:400-406.

126. Nys GM, van Zandvoort MJ, van der Worp HB, de Haan EH, de Kort PL, Kappelle LJ. Early depressive symptoms after stroke: Neuropsychological correlates and lesion characteristics. $J$ Neurol Sci. 2005;228:27-33. 
127. Sharpe M, Hawton K, House A, Molyneux A, Sandercock P, Bamford J, et al. Mood disorder in long-term survivors of stroke: Associations with brain lesion location and volume. Psychol Med. 1990;20:815-828.

128. Sharpe M, Hawton K, Seagroatt V, Bamford J, House A, Molyneux A, et al. Depressive disorders in long-term survivors of stroke. Associations with demographic and social factors, functional status, and brain lesion volume. Br J Psychiatry. 1994;164:380-386.

129. Bendsen BB, Bendsen EB, Lauritzen L, Vilmar T, Bech P. Post-stroke patients in rehabilitation. The relationship between biological impairment (CT scanning), physical disability and clinical depression. Eur Psychiatry. 1997;12:399-404.

130. Huang Y, Chen W, Li Y, Wu X, Shi X, Geng D. Effects of antidepressant treatment on $n$-acetyl aspartate and choline levels in the hippocampus and thalami of post-stroke depression patients: A study using ${ }^{1} \mathrm{~h}$ magnetic resonance spectroscopy. Psychiatry Res. 2010;182:48-52.

131. Xu QG, Cao H, Song OW, Wu JL. Metabolic changes of prefrontal cerebral lobe, white matter and cerebellum in patients with post-stroke depression: A proton magnetic resonance spectroscopy study. Neural Regen Res. 2008;3:142-147.

132. Glodzik-Sobanska L, Slowik A, McHugh P, Sobiecka B, Kozub J, Rich KE, et al. Single voxel proton magnetic resonance spectroscopy in post-stroke depression. Psychiatry Res. 2006;148:111-120.

133. Wang X, Li Y-H, Li M-H, Lu J, Zhao J-G, Sun X-J, et al. Glutamate level detection by magnetic resonance spectroscopy in patients with post-stroke depression. Eur Arch Psychiatry Clin Neurosci. 2012;262:33-38.

134. Fu JH, Wong K, Mok V, Hu X, Xiong Y, Chen Y, et al. Neuroimaging predictors for depressive symptoms in cerebral small vessel disease. Int J Geriatr Psychiatry. 2010;25:1039-1043.

135. Tang WK, Chen YK, Lu JY, Mok VCT, Chu WCW, Ungvari GS, et al. Frontal lobe atrophy in depression after stroke. Stroke Res Treat. 2013;424769.

136. Starkstein SE, Robinson RG, Price TR. Comparison of patients with and without poststroke major depression matched for size and location of lesion. Arch Gen Psychiatry. 1988;45:247-252.

137. Wichowicz HM, Gasecki D, Landowski J, Nyka WM, Kozera G, Cubala WJ. Regional cerebral blood flow (spect) asymmetry as a prognostic factor for post-stroke depression: A preliminary observation. Neurol Psychiatry Brain Res. 2006;13:165-167.

138. Zhang $P, X u Q$, Dai J, Wang J, Zhang N, Luo Y. Dysfunction of affective network in post ischemic stroke depression: A resting-state functional magnetic resonance imaging study. Biomed Res Int. 2014;2014:846830.

139. Williamson J, Nyenhuis D, Stebbins GT, Lamb D, Simkus V, Sripathirathan K, et al. Regional differences in relationships between apparent white matter integrity, cognition and mood in patients with ischemic stroke. J Clin Exp Neuropsychology. 2010;32:673-681.

140. Yasuno F, Taguchi A, Yamamoto A, Kajimoto K, Kazui H, Sekiyama A, et al. Microstructural abnormalities in white matter and their effect on depressive symptoms after stroke. Psychiatry Res. 2014;223:9-14.

141. Glodzik-Sobanska L, Slowik A, Kieltyka A, Kozub J, Sobiecka B, Urbanik A, et al. Reduced prefrontal n-acetylaspartate in stroke patients with apathy. J Neurol Sci. 2005;238:19-24.

142. Okada K, Kobayashi S, Yamagata S, Takahashi K, Yamaguchi S. Poststroke apathy and regional cerebral blood flow. Stroke. 1997;28:2437-2441.

143. Yang SR, Shang XY, Tao J, Liu JY, Hua P. Voxel-based analysis of fractional anisotropy in post-stroke apathy. PLOS ONE. 2015;10:e116168.

144. Chen Y-K, Qu J-F, Xiao W-M, Li W-Y, Li W, Fang X-W, et al. Intracranial atherosclerosis and poststroke depression in chinese patients with ischemic stroke. J Stroke Cerebrovasc Dis. 2016;25:998-1004.

145. Yang S, Hua P, Shang X, Cui Z, Zhong S, Gong G, et al. Deficiency of brain structural sub-network underlying post-ischaemic stroke apathy. Eur J Neurol. 2015;22:341-347.

146. Terroni L, Amaro E, losifescu DV, Tinone G, Sato JR, Leite CC, et al. Stroke lesion in cortical neural circuits and post-stroke incidence of major depressive episode: A 4-month prospective study. World J Biol Psychiatry. 2011;12:539-548. 


\section{Chapter 2}

147. Vataja R, Pohjasvaara T, Leppavuori A, Mantyla R, Aronen HJ, Salonen O, et al. Magnetic resonance imaging correlates of depression after ischemic stroke. Arch Gen Psychiatry. 2001;58:925-931.

148. Tang WK, Lu JY, Chen YK, Chu WCW, Mok V, Ungvari GS, et al. Association of frontal subcortical circuits infarcts in poststroke depression: A magnetic resonance imaging study of 591 chinese patients with ischemic stroke. J Geriatr Psychiatry Neurol. 2011;24:44-49.

149. Zhang $Y$, Cheng $L$, Chen $Y$, Yang G-Y, Liu J, Zeng L. Clinical predictor and circulating microrna profile expression in patients with early onset post-stroke depression. J Affect Disord. 2016;193:51-58.

150. Wei C, Zhang F, Chen L, Ma X, Zhang N, Hao J. Factors associated with post-stroke depression and fatigue: Lesion location and coping styles. J Neurol. 2016;263:269-276.

151. Gozzi SA, Wood AG, Chen J, Vaddadi K, Phan TG. Imaging predictors of poststroke depression: Methodological factors in voxel-based analysis. BMJ Open. 2014;4:e004948.

152. Chen Y, Chen LF, Tao YQ, Zhou FX, Cui CL, Liu SC. Investigation of prevalence and risk factors of depressive symptoms following acute ischemic stroke in the aged. J Am Geriatr Soc. 2013;61:S358. 


\section{SUPPLEMENTAL DATA}

Supplementary Table 1. Search terms used in five databases

\begin{tabular}{|c|c|c|}
\hline \multirow[b]{4}{*}{ 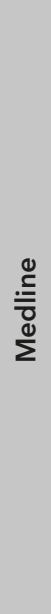 } & \multirow{4}{*}{$\begin{array}{l}\text { Platform } \\
\text { Database } \\
\text { Limits }\end{array}$} & OvidSP \\
\hline & & MEDLINE ${ }^{\circledR}$ In-Process \& Other Non-Indexed Citations \\
\hline & & Human subjects, adult subjects, language (German, Dutch, English, French) \\
\hline & & $\begin{array}{l}\text { (ischem* OR ischaem* OR stroke OR infarct* OR hemorrhag* OR haemorrhag* OR } \\
\text { lesion).ti,kf,hw } \\
\text { AND } \\
\text { (apath* OR depress* OR mood OR motivation OR neuropsychia* OR emotion OR } \\
\text { abulia).ti,kf,hw } \\
\text { AND } \\
\text { (MRI OR (magnetic AND resonance) OR imaging OR SPECT OR (Single-photon } \\
\text { AND Emission AND Positron AND Computed AND Tomography) OR fMRI OR } \\
\text { (functional AND MRI) OR PET OR (Positron AND Emission AND Tomography) } \\
\text { OR CT OR (Computed AND tomography) OR DTI OR (diffusion AND tensor) OR } \\
\text { connectivity).mp }\end{array}$ \\
\hline \multirow[b]{4}{*}{ 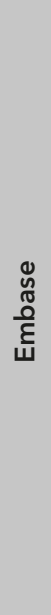 } & \multirow{4}{*}{$\begin{array}{l}\text { Platform } \\
\text { Database } \\
\text { Limits }\end{array}$} & OvidSP \\
\hline & & Embase 1980-present \\
\hline & & Human subjects, adult subjects, language (German, Dutch, English, French) \\
\hline & & $\begin{array}{l}\text { (ischem* OR ischaem* OR stroke OR infarct* OR hemorrhag* OR haemorrhag* OR } \\
\text { lesion).ti,kw, hw } \\
\text { AND } \\
\text { (apath* OR depress* OR mood OR motivation OR neuropsychia* OR emotion OR } \\
\text { abulia).ti,kw, hw } \\
\text { AND } \\
\text { (MRI OR (magnetic AND resonance) OR imaging OR SPECT OR (Single-photon } \\
\text { AND Emission AND Positron AND Computed AND Tomography) OR fMRI OR } \\
\text { (functional AND MRI) OR PET OR (Positron AND Emission AND Tomography) } \\
\text { OR CT OR (Computed AND tomography) OR DTI OR (diffusion AND tensor) OR } \\
\text { connectivity).mp }\end{array}$ \\
\hline \multirow[b]{4}{*}{$\frac{0}{\frac{1}{z}}$} & \multirow{4}{*}{$\begin{array}{l}\text { Platform } \\
\text { Database } \\
\text { Limits }\end{array}$} & EBSCOhost \\
\hline & & PsycINFO 1806 - present \\
\hline & & Human subjects, adult subjects, language (German, Dutch, English, French) \\
\hline & & $\begin{array}{l}\text { (ischem* OR ischaem* OR stroke OR infarct* OR haemorrhag* OR hemorrhage* or } \\
\text { lesion)SU } \\
\text { AND } \\
\text { (apath* OR depress* OR mood OR motivation OR neuropsychia* OR emotion OR } \\
\text { abulia)SU } \\
\text { AND } \\
\text { (MRI OR magnetic OR resonance OR imaging OR computed OR CT)TX }\end{array}$ \\
\hline
\end{tabular}




\begin{tabular}{|c|c|c|}
\hline \multirow[b]{4}{*}{ 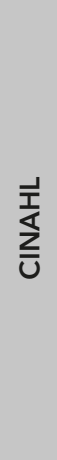 } & Platform & EBSCOhost \\
\hline & Database & CINAHL 1981 - present \\
\hline & Limits & Human subjects, adult subjects, language (German, Dutch, English, French) \\
\hline & Search & $\begin{array}{l}\text { (ischem* OR ischaem* OR stroke OR infarct* OR hemorrhage* OR haemorrhag* } \\
\text { OR lesion)SU } \\
\text { AND } \\
\text { (apath* OR depress* OR mood OR motivation OR neuropsychia* OR emotion OR } \\
\text { abulia)SU } \\
\text { AND } \\
\text { (MRI OR magnetic OR resonance OR imaging OR computed OR CT)TX }\end{array}$ \\
\hline \multirow[b]{4}{*}{ 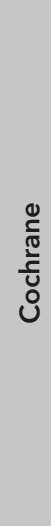 } & \multirow{4}{*}{$\begin{array}{l}\text { Platform } \\
\text { Database } \\
\text { Limits }\end{array}$} & Cochrane \\
\hline & & Wiley Online Library \\
\hline & & Human subjects, adult subjects, language (German, Dutch, English, French) \\
\hline & & $\begin{array}{l}\text { (ischem* OR ischaem* OR stroke OR infarct* OR haemorrhag* OR hemorrhage* } \\
\text { OR lesion) } \\
\text { AND } \\
\text { (apath* OR depress* OR mood OR motivation OR neuropsychia* OR emotion OR } \\
\text { abulia) } \\
\text { AND } \\
\text { (MRI OR (magnetic AND resonance) OR imaging OR CT OR (Computed } \\
\text { AND tomography) OR DTI OR (diffusion AND tensor) OR connectivity) TITLE/ } \\
\text { ABSTRACT/KEYWORDS }\end{array}$ \\
\hline
\end{tabular}


Supplementary Table 2. Contingency table data of meta-analyses separate for post-stroke depression and post-stroke apathy studies

\begin{tabular}{|c|c|c|c|c|c|c|c|c|c|c|c|c|c|c|c|c|c|c|c|c|}
\hline \multicolumn{21}{|c|}{ PSD studies } \\
\hline \multirow{3}{*}{ Authors } & \multicolumn{4}{|c|}{ Laterality } & \multicolumn{4}{|c|}{ Lesion type } & \multicolumn{4}{|c|}{ Frontal lesion } & \multicolumn{4}{|c|}{$\begin{array}{c}\text { Subcortical } \\
\text { lesion }\end{array}$} & \multicolumn{4}{|c|}{$\begin{array}{c}\text { Basal ganglia } \\
\text { lesion }\end{array}$} \\
\hline & \multicolumn{2}{|c|}{ ڤิ } & \multicolumn{2}{|c|}{ 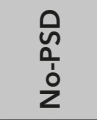 } & \multicolumn{2}{|c|}{ ڤ } & \multicolumn{2}{|c|}{$\begin{array}{l}0 \\
n \\
\vdots \\
z\end{array}$} & \multicolumn{2}{|c|}{ ڤิ } & \multicolumn{2}{|c|}{$\begin{array}{l}0 \\
\text { ñ } \\
\text { ò }\end{array}$} & \multicolumn{2}{|c|}{ ถุ } & \multicolumn{2}{|c|}{$\begin{array}{l}0 \\
\text { ñ } \\
\text { ò }\end{array}$} & \multicolumn{2}{|c|}{ ڤิ } & \multicolumn{2}{|c|}{$\begin{array}{l}0 \\
\vdots \\
\vdots \\
2\end{array}$} \\
\hline & Ф) & $\frac{\text { ț }}{\frac{\sigma}{\alpha}}$ & $\frac{\square}{ \pm}$ & $\frac{\ddot{n}}{\frac{\pi}{\alpha}}$ & $\frac{\varepsilon}{1}$ & 总 & $\frac{\varepsilon}{1}$ & 든 & 亡े & 亡 & 亡ั & 芒 & $\begin{array}{l}u \\
\text { ज̆ }\end{array}$ & $\begin{array}{l}\text { U } \\
\frac{\tilde{n}}{Z}\end{array}$ & $\frac{u}{3}$ & $\begin{array}{l}u \\
\text { o } \\
\text { un } \\
Z\end{array}$ & ن্ & U్ల & ৩ & U్ \\
\hline \multicolumn{21}{|c|}{ Acute stroke phase } \\
\hline Robinson et al. (1984a) & 11 & 2 & 7 & 10 & - & - & - & - & - & - & - & - & - & - & - & - & - & - & - & - \\
\hline Starkstein et al. (1989) & - & - & - & - & 2 & 14 & 3 & 22 & 7 & 10 & 5 & 20 & - & - & $\cdots$ & $\cdots$ & 3 & 14 & 6 & 19 \\
\hline Starkstein et al. (1991) & 18 & 8 & 14 & 16 & - & - & - & - & - & - & - & - & - & - & - & - & - & - & - & - \\
\hline Astrom et al. (1993)* & 14 & 2 & 7 & 21 & - & - & - & - & 12 & 2 & 2 & 5 & - & - & - & $\begin{array}{c}- \\
\cdots\end{array}$ & - & - & - & - \\
\hline Morris et al. (1996a) & 21 & 26 & 56 & 90 & - & - & - & - & - & - & - & - & - & - & - & - & - & - & - & - \\
\hline Huwel et al. (1998) & 12 & 18 & 14 & 11 & - & - & - & - & - & - & - & - & - & - & - & - & 3 & 27 & 5 & 20 \\
\hline Gainotti et al. (1999) & 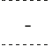 & - & - & - & $\begin{array}{c}- \\
-\end{array}$ & $\begin{array}{c}- \\
\cdots\end{array}$ & - & - & 4 & 11 & 12 & 26 & - & - & - & $\begin{array}{c}- \\
-\cdots\end{array}$ & - & - & - & - \\
\hline Paradiso et al. (1999) & 32 & 22 & 31 & 53 & 8 & 46 & 22 & 62 & 37 & 17 & 63 & 21 & 26 & 28 & 38 & 46 & - & - & 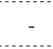 & - \\
\hline Berg et al. (2001) & 11 & 6 & 21 & 31 & - & - & - & - & - & - & - & - & - & - & - & - & - & - & - & - \\
\hline Hsieh and Kao (2005) & 32 & 39 & 54 & 82 & - & - & - & - & - & - & - & - & - & - & - & - & - & - & - & - \\
\hline Nys et al. (2005) & 25 & 41 & 28 & 32 & 10 & 56 & 5 & 55 & - & - & $\begin{array}{c}- \\
\cdots\end{array}$ & - & - & - & - & - & - & - & - & - \\
\hline Glodzik-Sob. et al. (2006)* & 4 & 4 & 11 & 7 & - & - & - & - & - & - & - & - & 8 & 0 & 14 & 4 & - & - & - & - \\
\hline Caeiro et al. (2006) & 21 & 26 & 22 & 29 & 25 & 57 & 32 & 64 & 5 & 7 & 8 & 10 & 17 & 65 & 22 & 74 & - & - & - & - \\
\hline Wongwandee (2012) & 8 & 3 & 13 & 15 & - & - & - & - & 0 & 11 & 1 & 27 & - & - & - & - & 9 & 2 & 12 & 16 \\
\hline Choi-Kwon et al. (2012)* & 29 & 41 & 203 & 235 & - & - & - & - & 16 & 54 & 71 & 367 & - & - & - & - & - & - & - & - \\
\hline Chen et al. (2013) & 13 & 18 & 29 & 42 & - & - & - & - & - & - & - & - & - & - & - & - & - & - & - & - \\
\hline Zhang et al. (2013) & 9 & 16 & 26 & 40 & 1 & 24 & 6 & 60 & - & - & - & - & - & - & - & - & - & - & - & - \\
\hline Shi et al. (2014) & $\begin{array}{c}\cdots \\
\cdots\end{array}$ & - & - & - & $\begin{array}{c}- \\
-\end{array}$ & - & - & - & 36 & 267 & 73 & 691 & - & - & $\begin{array}{c}- \\
-\end{array}$ & - & - & - & - & - \\
\hline Terroni et al. (2015) & 6 & 1 & 15 & 14 & $\cdots$ & $\begin{array}{c}- \\
\cdots\end{array}$ & - & - & - & $\cdots$ & - & - & - & - & $\cdots$ & $\cdots$ & - & - & - & - \\
\hline Saxena \& Suman (2015) & 42 & 19 & 19 & 27 & 10 & 51 & 4 & 42 & - & - & - & - & - & - & - & - & - & - & - & - \\
\hline Metoki et al. (2016) & 59 & 85 & 220 & 248 & - & $\begin{array}{ll}- \\
\cdots\end{array}$ & - & - & 7 & 64 & 14 & 336 & - & - & $\begin{array}{c}- \\
\cdots\end{array}$ & - & 10 & 61 & 25 & 325 \\
\hline Chen et al. (2016)* & 29 & 33 & 46 & 34 & $\begin{array}{c}- \\
-\end{array}$ & $\begin{array}{c}- \\
\cdots\end{array}$ & $\begin{array}{c}- \\
\cdots\end{array}$ & - & 13 & 72 & 19 & 103 & - & - & $\begin{array}{c}- \\
-\end{array}$ & $\begin{array}{c}\cdots \\
\cdots\end{array}$ & 18 & 67 & 21 & 101 \\
\hline Wei et al. $(2016)^{\star}$ & 33 & 38 & 133 & 164 & - & - & - & - & 15 & 56 & 71 & 226 & - & - & - & - & - & - & - & - \\
\hline Zhang et al. (2016) & 19 & 26 & 109 & 97 & - & - & - & - & 2 & 43 & 13 & 193 & - & - & - & - & 21 & 24 & 103 & 3103 \\
\hline \multicolumn{21}{|c|}{ Post-acute stroke phase } \\
\hline Eastwood et al. (1989) & 14 & 33 & 12 & 20 & - & - & - & - & - & - & - & - & - & - & - & - & - & - & - & - \\
\hline Morris et al. (1990) & 18 & 16 & 24 & 30 & 0 & 14 & 7 & 14 & - & - & - & - & - & - & - & - & - & - & - & - \\
\hline House et al. (1990)* & 4 & 7 & 29 & 23 & - & - & - & - & 5 & 2 & 16 & 40 & - & - & - & - & - & - & - & - \\
\hline Morris et al. (1992) & $\begin{array}{c}- \\
\cdots\end{array}$ & - & - & - & - & $\begin{array}{c}- \\
\cdots\end{array}$ & - & - & - & $\begin{array}{c}- \\
-\cdots\end{array}$ & - & - & - & - & - & - & - & - & - & - \\
\hline Schwartz et al. (1993) & 7 & 29 & 21 & 34 & - & - & - & - & - & - & - & - & - & - & - & - & - & - & - & - \\
\hline Astrom et al. (1993)* & 11 & 8 & 11 & 14 & - & - & - & - & - & - & - & - & - & - & - & - & - & - & - & - \\
\hline González. et al. (1995) & 23 & 25 & 36 & 46 & - & - & - & - & - & - & - & - & - & - & - & - & - & - & - & - \\
\hline
\end{tabular}




\section{PSD studies}

Authors

\begin{tabular}{lcccc}
\multicolumn{3}{c}{ PSD studies } & \\
Laterality & Lesion type & Frontal lesion & $\begin{array}{c}\text { Subcortical } \\
\text { lesion }\end{array}$ & $\begin{array}{c}\text { Basal ganglia } \\
\text { lesion }\end{array}$
\end{tabular}

\begin{tabular}{|c|c|c|c|c|c|c|c|c|}
\hline ڤิ & 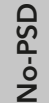 & ڤิ & $\begin{array}{l}0 \\
\text { ڤุ } \\
\text { o }\end{array}$ & ஸि & $\begin{array}{l}\text { ڤิ } \\
\text { ò } \\
\text { z }\end{array}$ & ڤิ & $\begin{array}{l}0 \\
\text { ñ } \\
\text { ò }\end{array}$ & ص \\
\hline
\end{tabular}

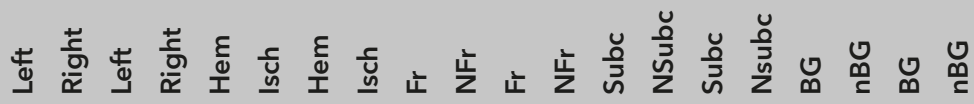

Herrmann et al. (1995)

$\begin{array}{llll}12 & 5 & 20 & 10\end{array}$

Andersen et al. (1995)

$\begin{array}{llll}15 & 22 & 84 & 71\end{array}$

$\begin{array}{llllllll}12 & 16 & 24 & 67 & 21 & 7 & 66 & 25\end{array}$

Ng et al. (1995)

$\begin{array}{llllllll}4 & 25 & 8 & 15 & 13 & 16 & 2 & 21\end{array}$

Bendsen et al. (1997)

$\begin{array}{llll}13 & 7 & 49 & 59\end{array}$

Gainotti et al. (1997)

$\begin{array}{llll}16 & 34 & 24 & 52\end{array}$

MacHale et al. (1998)

$\begin{array}{llll}3 & 10 & 27 & 15\end{array}$

Kase et al. (1998)

$\begin{array}{llll}10 & 11 & 12 & 11\end{array}$

Pohjasvaara et al. (1998) $65 \quad 46 \quad 86 \quad 80$

Paolucci et al. (1999)

5175161178

$41 \quad 85124215$

Gainotti et al. (1999)

$\begin{array}{llll}5 & 7 & 8 & 26\end{array}$

Singh et al. (2000)

$\begin{array}{llll}10 & 19 \quad 4 \quad 48\end{array}$

Kim et al. (2000)

$\begin{array}{llll}5 & 24 & 25 & 27\end{array}$

Gainotti et al. (2001)

Spalletta et al. (2002)

$\begin{array}{llllllllllll}11 & 16 & 69 & 52 & 2 & 25 & 20 & 101 & 24 & 3 & 52 & 69\end{array}$

Desmond et al. (2003)

1730124250

$\begin{array}{llllllllllll}16 & 72 & 20 & 45 & 21 & 51 & 14 & 31 & 32 & 40 & 16 & 29\end{array}$

Verdelho et al. (2004)*

- $\quad-\quad-640755$

$\begin{array}{llll}10 & 37 \quad 81293\end{array}$

Spalletta et al. (2005)

$\begin{array}{lllr}43 & 69 & 34 & 54\end{array}$

Tang et al. (2005)

Glodzik-Sob. et al. $(2006)^{\star} \quad 7 \quad 2 \quad 8 \quad 9$

Brodaty et al. (2007) $17 \quad 20 \quad 47 \quad 51$

Fuentes et al. (2009) $\quad 5 \quad 12 \quad 23 \quad 19$

$\begin{array}{lllll}\text { Oladiji et al. (2009) } & 3 & 10 & 17 & 21\end{array}$

$\begin{array}{lllll}\text { Snaphaan et al. (2009) } & 21 & 21 & 99 & 142\end{array}$

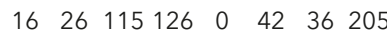

Sienkiewicz et al. (2010) $61 \quad 21 \quad 121 \quad 39 \quad 8 \quad 74 \quad 16 \quad 144$

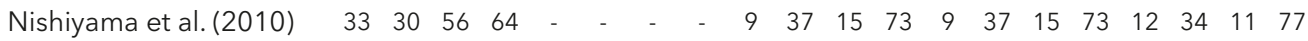

Bour et al. (2010)

Tang et al. (2010)

$\begin{array}{llll}11 & 15 & 51 & 61\end{array}$

Effat et al. (2011)

$\begin{array}{llll}20 & 58 & 16 & 62\end{array}$

Terroni et al. (2011)

$\begin{array}{llll}13 & 8 & 28 & 19\end{array}$

Tang et al. (2011a)

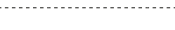

Tennen et al. (2011)

$\begin{array}{llll}21 & 17 \quad 36 \quad 28\end{array}$

Choi-Kwon et al. (2012)* $35 \quad 48 \quad 182204$

$\begin{array}{llll}17 & 10 & 71 & 83\end{array}$

$\begin{array}{llll}9 & 14 & 11 & 26\end{array}$

$\begin{array}{llll}11 & 12 & 9 & 28\end{array}$

Altieri et al. (2012)

$\begin{array}{llll}22 & 21 & 35 & 27\end{array}$ 


\begin{tabular}{|c|c|c|c|c|c|c|c|c|c|c|c|c|c|c|c|c|c|c|c|c|}
\hline \multicolumn{21}{|c|}{ PSD studies } \\
\hline \multirow{3}{*}{ Authors } & \multicolumn{4}{|c|}{ Laterality } & \multicolumn{4}{|c|}{ Lesion type } & \multicolumn{4}{|c|}{ Frontal lesion } & \multicolumn{4}{|c|}{$\begin{array}{c}\text { Subcortical } \\
\text { lesion }\end{array}$} & \multicolumn{4}{|c|}{$\begin{array}{c}\text { Basal ganglia } \\
\text { lesion }\end{array}$} \\
\hline & \multicolumn{2}{|c|}{ ถุ } & \multicolumn{2}{|c|}{$\begin{array}{l}0 \\
n \\
\vdots \\
z\end{array}$} & \multicolumn{2}{|c|}{ ฉ } & \multicolumn{2}{|c|}{$\begin{array}{l}0 \\
\text { ñ } \\
\text { o }\end{array}$} & \multicolumn{2}{|c|}{ ฉ } & \multicolumn{2}{|c|}{$\begin{array}{l}0 \\
n \\
\vdots \\
z\end{array}$} & \multicolumn{2}{|c|}{ ำ } & \multicolumn{2}{|c|}{$\begin{array}{l}0 \\
\text { ñ } \\
\text { ò }\end{array}$} & \multicolumn{2}{|c|}{ ڤิ } & \multicolumn{2}{|c|}{$\begin{array}{l}\text { ڤั } \\
\text { ò }\end{array}$} \\
\hline & 齿 & $\frac{t}{\frac{}{0}}$ & ए & $\frac{\text { ㄹ }}{\frac{0}{\alpha}}$ & $\frac{\varepsilon}{1}$ & 든 & $\frac{\varepsilon}{1}$ & 든 & 亡 & 芒 & 亡․ & 市 & $\begin{array}{l}u \\
\frac{0}{2} \\
\text { n }\end{array}$ & $\begin{array}{l}u \\
\frac{0}{5} \\
\text { U }\end{array}$ & uั & $\begin{array}{l}\text { U } \\
\frac{0}{3} \\
\text { Z }\end{array}$ & ర్ల & 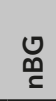 & ৩ு & ֻ \\
\hline Zhang et al. (2012) & 17 & 22 & 43 & 81 & - & - & - & - & 14 & 25 & 24 & 100 & - & - & - & - & - & - & - & - \\
\hline Rajashekaran et al. (2013) & 19 & 9 & 8 & 26 & - & - & - & - & - & - & - & - & - & - & - & - & - & - & - & - \\
\hline Gozzi et al. (2014) & 7 & 8 & 20 & 20 & - & - & - & - & 8 & 7 & 24 & 16 & - & - & $\begin{array}{c}- \\
\cdots\end{array}$ & $\begin{array}{c}- \\
\cdots\end{array}$ & - & - & - & - \\
\hline Jiang et al. (2014) & 50 & 48 & 60 & 171 & 11 & 87 & 20 & 211 & 22 & 76 & 19 & 212 & - & - & - & - & 86 & 12 & 116 & 6115 \\
\hline Shi et al. (2014) & - & - & - & - & - & - & - & - & 33 & 187 & 76 & 771 & - & - & - & - & - & - & - & - \\
\hline Gu et al. (2015) & - & - & - & - & - & - & - & - & 9 & 47 & 21 & 119 & - & - & - & - & 17 & 39 & 32 & 108 \\
\hline Wichowicz et al. (2015) & 21 & 8 & 42 & 30 & - & - & - & - & 18 & 11 & 23 & 49 & - & - & - & - & 15 & 14 & 19 & 53 \\
\hline Chen et al. $(2016)^{\star}$ & 26 & 26 & 42 & 37 & - & - & - & - & 9 & 64 & 20 & 100 & - & - & $\begin{array}{c}\cdots \\
-\cdots\end{array}$ & 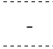 & 15 & 58 & 21 & 99 \\
\hline Wei et al. (2016)* & 40 & 47 & 126 & 155 & - & - & - & - & 24 & 63 & 62 & 219 & - & - & - & - & - & - & - & - \\
\hline \multicolumn{21}{|c|}{ Chronic stroke phase } \\
\hline House et al. $(1990)^{\star}$ & 1 & 4 & 30 & 23 & - & - & - & - & - & - & - & - & - & - & - & - & - & - & - & - \\
\hline Sharpe et al. (1990) & 6 & 5 & 25 & 24 & - & - & - & - & - & - & - & - & - & - & - & - & - & - & - & - \\
\hline Astrom et al. (1993)* & 5 & 4 & 16 & 15 & - & - & - & - & - & - & - & - & - & - & - & - & - & - & - & - \\
\hline Verdelho et al. (2004)* & - & - & - & - & 1 & 12 & 8 & 52 & - & $\begin{array}{c}- \\
\cdots\end{array}$ & - & - & 9 & 4 & 40 & 20 & - & - & - & - \\
\hline Provinciali et al. (2008) & 144 & 120 & 234 & 233 & - & - & - & - & - & - & - & - & - & - & - & - & - & - & - & - \\
\hline Chatterjee et al. (2010) & 12 & 28 & 20 & 67 & - & - & - & - & - & - & - & - & 16 & 17 & 20 & 50 & - & - & - & - \\
\hline Nidhinandana et al. (2010) & 17 & 30 & 22 & 32 & - & - & - & - & - & - & - & - & - & - & - & - & - & - & - & - \\
\hline Srivastava et al. (2010) & 8 & 10 & 16 & 17 & 3 & 15 & 6 & 27 & - & - & - & - & - & - & - & - & - & - & - & \\
\hline
\end{tabular}

\begin{tabular}{|c|c|c|c|c|c|c|c|c|c|c|c|c|c|c|c|c|c|c|c|c|}
\hline \multicolumn{21}{|c|}{ PSA studies } \\
\hline \multirow{3}{*}{ Authors } & \multicolumn{4}{|c|}{ Laterality } & \multicolumn{4}{|c|}{ Lesion type } & \multicolumn{4}{|c|}{ Frontal lesion } & \multicolumn{4}{|c|}{$\begin{array}{c}\text { Subcortical } \\
\text { lesion }\end{array}$} & \multicolumn{4}{|c|}{$\begin{array}{c}\text { Basal ganglia } \\
\text { lesion }\end{array}$} \\
\hline & \multicolumn{2}{|c|}{ ఓ్ } & \multicolumn{2}{|c|}{$\begin{array}{l}\overleftarrow{c} \\
\vdots \\
\vdots \\
z\end{array}$} & \multicolumn{2}{|c|}{ « } & \multicolumn{2}{|c|}{ 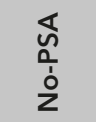 } & \multicolumn{2}{|c|}{ ఓ } & \multicolumn{2}{|c|}{$\begin{array}{l}\overleftarrow{1} \\
\vdots \\
\vdots \\
2\end{array}$} & \multicolumn{2}{|c|}{ 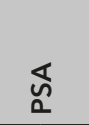 } & \multicolumn{2}{|c|}{$\begin{array}{l}\frac{1}{1} \\
\vdots \\
\vdots \\
z\end{array}$} & \multicolumn{2}{|c|}{ ఓ } & \multicolumn{2}{|l|}{$\begin{array}{l}\overleftarrow{c} \\
\vdots \\
\vdots \\
z\end{array}$} \\
\hline & $\frac{ \pm}{9}$ & $\frac{ \pm}{\frac{\pi}{0}}$ & $\frac{ \pm}{9}$ & $\frac{\ddot{z}}{\frac{0}{x}}$ & $\begin{array}{l}\varepsilon \\
\text { I } \\
\end{array}$ & 造 & $\frac{\varepsilon}{0}$ & $\underline{\underline{u}}$ & 立 & 立 & 호 & 亡 & $\stackrel{u}{\frac{0}{3}}$ & $\begin{array}{l}u \\
\frac{0}{5} \\
\text { Z }\end{array}$ & $\frac{u}{3}$ & $\begin{array}{l}u \\
\text { On } \\
\text { Z }\end{array}$ & ర్ల & 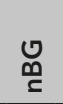 & ৩ & ֻૃ \\
\hline \multicolumn{21}{|c|}{ Acute stroke phase } \\
\hline Starkstein et al. (1993) & 6 & 8 & 10 & 34 & 5 & 9 & 10 & 34 & 3 & 11 & 7 & 37 & - & - & - & - & 6 & 8 & 152 & 29 \\
\hline Piamarta et al. (2004) & 3 & 2 & 16 & 12 & - & - & - & - & - & - & - & - & - & - & - & - & - & - & - & - \\
\hline Carota et al. (2005) & 44 & 53 & 78 & 54 & - & - & - & - & - & - & - & - & - & - & - & - & - & - & - & - \\
\hline Glodzik-Sob. et al. (2005) & 6 & 7 & 10 & 8 & - & - & - & - & - & - & - & - & 8 & 5 & 10 & 8 & - & - & - & - \\
\hline Kang and Kim (2008) & 28 & 9 & 27 & 27 & - & - & - & - & - & - & - & - & - & - & - & - & - & - & - & - \\
\hline Caeiro et al. (2012) & 7 & 20 & 24 & 17 & 13 & 23 & 9 & 49 & 4 & 5 & 3 & 8 & 9 & 9 & 17 & 11 & - & - & - & - \\
\hline
\end{tabular}




\section{PSA studies}

\begin{tabular}{|c|c|c|c|c|c|c|c|c|c|c|c|c|c|c|c|c|c|c|}
\hline \multirow{3}{*}{ Authors } & \multicolumn{4}{|c|}{ Laterality } & \multicolumn{4}{|c|}{ Lesion type } & \multicolumn{3}{|c|}{ Frontal lesion } & \multicolumn{4}{|c|}{$\begin{array}{l}\text { Subcortical } \\
\text { lesion }\end{array}$} & \multicolumn{3}{|c|}{$\begin{array}{c}\text { Basal ganglia } \\
\text { lesion }\end{array}$} \\
\hline & \multicolumn{2}{|c|}{ ๕్ } & \multicolumn{2}{|c|}{$\begin{array}{l}\nwarrow \\
\\
\dot{1}\end{array}$} & \multicolumn{2}{|c|}{ 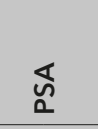 } & \multicolumn{2}{|c|}{ 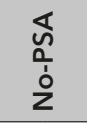 } & \multicolumn{2}{|c|}{$\overleftarrow{\nwarrow}$} & 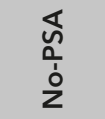 & \multicolumn{2}{|c|}{ 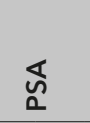 } & \multicolumn{2}{|c|}{$\begin{array}{l}\mathbb{1} \\
\vdots \\
\vdots \\
z\end{array}$} & \multicolumn{2}{|c|}{ « } & $\begin{array}{l}\ll \\
\\
\dot{0} \\
z\end{array}$ \\
\hline & $\frac{5}{9}$ & 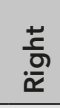 & $\frac{5}{9}$ & 蒙 & $\underset{\frac{\varepsilon}{\Phi}}{\underline{I}}$ & $\underline{\underline{w}}$ & $\begin{array}{l}\varepsilon \\
\stackrel{\varepsilon}{I}\end{array}$ & $\underline{\underline{\underline{v}}}$ & 立 & 芫 & 亡 芒 & $\frac{\breve{o}}{\vec{n}}$ & 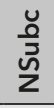 & 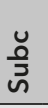 & $\begin{array}{l}\frac{u}{J} \\
\frac{\omega}{Z} \\
z\end{array}$ & ৩্ & ֻू & ט \\
\hline \multicolumn{19}{|c|}{ Post-acute stroke phase } \\
\hline Yamagata et al. (2004) & 7 & 9 & 4 & 9 & - & - & - & - & - & - & - & - & - & - & - & - & - & - \\
\hline Brodaty et al. (2005) & 4 & 13 & 10 & 50 & - & - & - & - & - & - & - & - & - & - & - & - & - & - \\
\hline Santa et al. (2008) & 8 & 6 & 19 & 34 & 3 & 11 & 29 & 24 & 5 & 41 & 30123 & - & - & - & - & 21 & 25 & 6192 \\
\hline Mikami et al. (2013) & - & - & - & - & 0 & 23 & 5 & 28 & - & - & $-\quad-$ & - & - & - & - & - & - & $-\quad-$ \\
\hline Tang et al. (2013a) & - & - & - & - & - & - & - & - & 2 & 18 & 22143 & - & - & - & - & 4 & 16 & 22143 \\
\hline Cosin et al. (2015) & - & - & - & - & - & - & - & - & 2 & 8 & 729 & - & - & - & - & 0 & 10 & 333 \\
\hline
\end{tabular}

*Studies that provided data on more than one time point. Note: Data represent number of patients.

Abbreviations: BG, basal ganglia lesion; Fr, frontal lesion; Hem, hemorrhage; Isch, ischemic stroke; nBG, non basal ganglia lesion; NFr, non-frontal lesion; NSubc, non-subcortical lesion; PSA, post-stroke apathy; PSD, post-stroke depression; Subc, subcortical lesion. 
Supplementary Table 3. Newcastle-Ottowa Quality Assessment Scale

Study

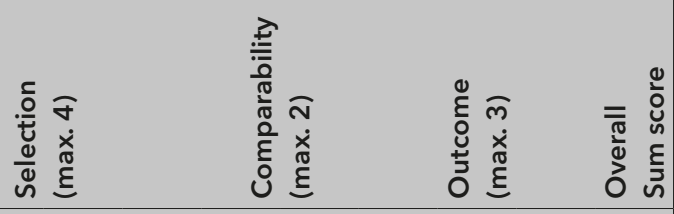

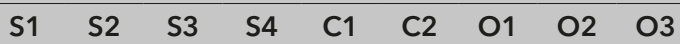

Cohort studies (PSD)

Robinson et al. (1984b);

Robinson et al. (1985b)

Parikh et al. (1988)

House et al. (1990)

Morris et al. (1990)

Astrom et al. (1993)

Andersen et al. (1995)

Herrmann et al. (1998)

Huwel et al. (1998)

Shimoda et al. (1999)

Singh et al. (2000)

Berg et al. (2003)

Cassidy et al. (2004)

Verdelho et al. (2004)

Paolucci et al. (2006);

Provinciali et al. (2008)

Aben et al. (2006); Leentjens et al.

(2006); Bour et al. (2010)

Fuentes et al. (2009)

Iranmanesh et al. (2009)

Terroni et al. (2011)

Kim et al. (2011)

Castellanos-Pinedo et al. (2011)

Altieri et al. (2012)

Choi-Kwon et al. (2012)

Ku et al. (2013)

Zhang et al. (2013)

Shi et al. (2014)

De Ryck et al. (2014)

Gozzi et al. (2014)

Tang et al. (2014a)

Wichowicz et al. (2015)

Wei et al. (2016)

Chen et al. (2016)

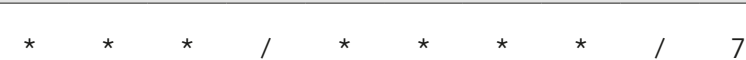

\begin{tabular}{|c|c|c|c|c|c|c|c|c|c|}
\hline$\star$ & $\star$ & $\star$ & / & / & / & $\star$ & $\star$ & / & 5 \\
\hline * & $\star$ & $\star$ & / & * & $\star$ & $\star$ & $\star$ & * & 8 \\
\hline$\star$ & $\star$ & $\star$ & / & $\star$ & $\star$ & $\star$ & $\star$ & / & 7 \\
\hline$\star$ & $\star$ & $\star$ & / & $\star$ & $\star$ & $\star$ & $\star$ & $\star$ & 8 \\
\hline$\star$ & $\star$ & * & * & $\star$ & * & * & $\star$ & $\star$ & 9 \\
\hline$\star$ & / & $\star$ & / & $\star$ & $\star$ & $\star$ & $\star$ & / & 6 \\
\hline$\star$ & $\star$ & $\star$ & / & * & $\star$ & $\star$ & $\star$ & $\star$ & 8 \\
\hline$\star$ & $\star$ & $\star$ & / & $\star$ & $\star$ & $\star$ & $\star$ & $\star$ & 8 \\
\hline$\star$ & $\star$ & * & $\star$ & / & / & $\star$ & $\star$ & / & 6 \\
\hline$\star$ & $\star$ & $\star$ & $\star$ & * & $\star$ & $\star$ & $\star$ & $\star$ & 9 \\
\hline$\star$ & / & $\star$ & / & / & $\star$ & $\star$ & $\star$ & $\star$ & 6 \\
\hline$\star$ & / & $\star$ & / & $\star$ & $\star$ & $\star$ & $\star$ & $\star$ & 7 \\
\hline
\end{tabular}


Study

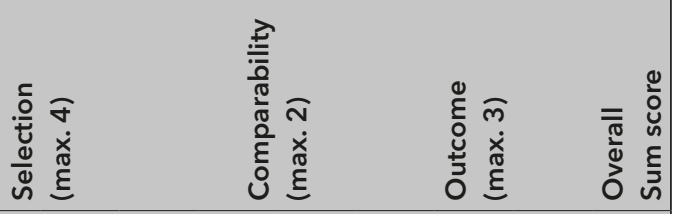

$\begin{array}{lllllllll}\mathrm{S} 1 & \mathrm{~S} 2 & \mathrm{~S} 3 & \mathrm{~S} 4 & \mathrm{C} 1 & \mathrm{C} 2 & \mathrm{O} 1 & \mathrm{O} 2 & \mathrm{O} 3\end{array}$

Case-control studies (PSD)

Eastwood et al. (1989)

Dam et al. (1989)

Kase et al. (1998)

Rao et al. (2001)

Desmond et al. (2003)

Nys et al. (2005)

Glodzik-Sobanska et al. (2006)

Caeiro et al. (2006)

Brodaty et al. (2007);

Withall et al. (2011)

Xu et al. (2008)

Huang et al. (2010)

Wang et al. (2012)

Yasuno et al. (2014)

Zhang et al. (2014)

Brookes et al. (2014)

Hollocks et al. (2015)

\begin{tabular}{|c|c|c|c|c|c|c|c|c|c|}
\hline / & * & / & * & * & I & * & * & * & 6 \\
\hline * & * & I & * & / & I & * & * & * & 6 \\
\hline * & * & * & * & * & * & * & * & * & 9 \\
\hline * & / & / & * & * & * & * & * & * & 7 \\
\hline * & * & * & / & * & * & * & * & * & 8 \\
\hline * & * & * & * & * & * & * & * & * & 9 \\
\hline * & * & * & * & * & * & * & * & * & 9 \\
\hline * & * & / & / & * & * & * & * & * & 7 \\
\hline * & * & * & * & * & * & * & * & * & 9 \\
\hline * & * & * & / & * & I & * & * & * & 7 \\
\hline * & * & * & * & * & * & / & / & * & 7 \\
\hline * & * & * & * & * & * & * & * & * & 9 \\
\hline * & * & * & * & * & * & * & * & * & 9 \\
\hline * & * & * & * & * & * & * & * & * & 9 \\
\hline * & * & * & * & * & * & / & * & * & 8 \\
\hline * & * & * & * & * & $\star$ & / & * & * & 8 \\
\hline
\end{tabular}

Study

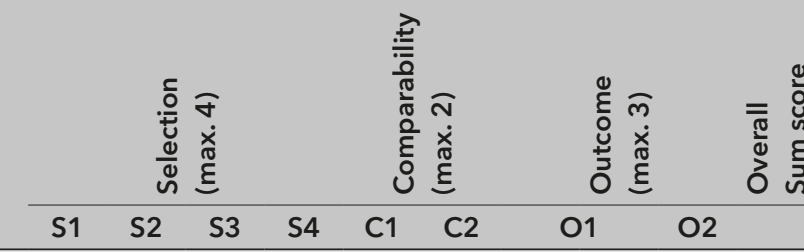

Cross-sectional studies (PSD)

Robinson et al. (1983)

Robinson et al. (1984a)

Robinson et al. (1985a)

Starkstein et al. (1988)

Starkstein et al. (1989)

Sharpe et al. (1990)

Sharpe et al. (1994)

Starkstein et al. (1991)

Stern et al. (1991)

Morris et al. (1992)

Morris et al. (1996b)

Schwartz et al. (1993)

Herrmann et al. (1993)

Cross-sectional studies (PSD)




\begin{tabular}{|c|c|c|c|c|c|c|c|c|c|}
\hline Herrmann et al. (1995) & * & / & * & * & * & * & ** & * & 8 \\
\hline $\mathrm{Ng}$ et al. (1996) & * & * & I & I & * & * & $\star \star$ & * & 7 \\
\hline Gonzalez-Torrecillas et al. (1995) & * & * & * & / & * & * & $\star \star$ & * & 8 \\
\hline Morris et al. (1996a) & * & * & * & I & * & * & $\star *$ & * & 8 \\
\hline Angeleri et al. (1997) & * & I & * & l & I & 1 & $\star \star$ & * & 5 \\
\hline Bendsen et al. (1997) & * & I & I & * & I & * & $\star *$ & * & 6 \\
\hline Gainotti et al. (1997) & * & I & * & * & l & 1 & $\star \star$ & I & 5 \\
\hline $\begin{array}{l}\text { Pohjasvaara et al. (1998); } \\
\text { Vataja et al. (2001); } \\
\text { Vataja et al. (2004) }\end{array}$ & * & * & * & / & / & / & ** & * & 6 \\
\hline MacHale et al. (1998) & * & * & I & l & * & I & ** & * & 6 \\
\hline Paolucci et al. (1999) & * & * & * & * & * & * & $\star *$ & * & 9 \\
\hline Paradiso et al. (1999) & * & * & * & I & * & * & $\star \star$ & * & 8 \\
\hline $\begin{array}{l}\text { Gainotti et al. (1999); } \\
\text { Gainotti et al. (2001) }\end{array}$ & * & / & * & * & * & * & ** & * & 8 \\
\hline Kim et al. (2000) & * & l & * & * & * & * & I & * & 7 \\
\hline Hosking et al. (2000) & * & * & * & * & * & * & I & * & 7 \\
\hline Berg et al. (2001) & * & * & * & * & * & * & ** & * & 9 \\
\hline $\begin{array}{l}\text { Spalletta et al. (2002) } \\
\text { Spalletta et al. (2005) }\end{array}$ & * & * & * & / & / & / & $\star \star$ & * & 6 \\
\hline Piamarta et al. (2004) & * & l & * & * & * & l & $\star *$ & l & 6 \\
\hline Kadojić et al. (2005) & * & / & * & * & / & * & / & / & 4 \\
\hline Aybek et al. (2005) & * & I & * & I & I & * & ** & I & 5 \\
\hline Hsieh et al. (2005) & * & / & * & * & * & * & $\star *$ & * & 8 \\
\hline Tang et al. (2005) & * & * & * & I & I & * & $\star \star$ & * & 7 \\
\hline Wichowicz et al. (2006) & * & / & * & * & / & / & $\star \star$ & I & 5 \\
\hline Barker-Collo et al. (2007) & * & * & * & I & * & * & I & * & 6 \\
\hline Hama et al. (2007) & * & * & * & * & l & I & I & * & 5 \\
\hline Oladiji et al. (2009) & * & * & * & l & * & * & I & * & 6 \\
\hline Snaphaan et al. (2009) & * & * & * & I & * & * & l & * & 6 \\
\hline Chen et al. (2009) & * & * & * & * & * & * & I & * & 7 \\
\hline Nishiyama et al. (2010) & * & * & * & * & * & * & I & * & 7 \\
\hline Chau et al. (2010) & * & l & * & I & * & * & I & * & 5 \\
\hline Mok et al. (2010) & * & / & * & * & * & * & ** & * & 8 \\
\hline Fu et al. (2010) & * & I & * & * & * & * & l & * & 6 \\
\hline Sienkiewicz-Jarosz et al. (2010) & * & / & * & I & * & * & I & * & 5 \\
\hline Williamson et al. (2010) & * & * & * & I & * & * & 1 & * & 6 \\
\hline Nidhinandana et al. (2010) & * & * & I & * & * & * & I & * & 6 \\
\hline Srivastava et al. (2010) & * & * & I & I & * & * & $\star \star$ & * & 7 \\
\hline Chatterjee et al. (2010) & * & * & * & I & * & * & $\star \star$ & * & 8 \\
\hline $\begin{array}{l}\text { Tang et al. (2010); Tang et al. (2011 b); } \\
\text { Tang et al. (2011c); } \\
\text { Tang et al. (2013b) }\end{array}$ & * & / & * & * & * & * & ** & * & 8 \\
\hline Tang et al. (2011a) & * & 1 & * & * & * & * & / & * & 6 \\
\hline
\end{tabular}




\begin{tabular}{|c|c|c|c|c|c|c|c|c|c|c|}
\hline Effat et al. (2011) & * & I & * & * & * & * & & *夫 & * & 8 \\
\hline Tennen et al. (2011) & * & * & * & l & * & * & & I & * & 6 \\
\hline Marasco et al. (2011) & * & * & * & l & * & * & & ** & * & 8 \\
\hline Zhang et al. (2012) & * & $\star$ & * & * & * & * & & ** & * & 9 \\
\hline Wongwandee et al. (2012) & $\star$ & / & * & $\star$ & $\star$ & $\star$ & & $\star \star$ & * & 8 \\
\hline Chen et al. (2013) & * & / & * & l & * & * & & $\star *$ & * & 7 \\
\hline Rajashekaran et al. (2013) & * & * & * & l & * & * & & $\star \star$ & * & 8 \\
\hline Taylor-Pillae et al. (2013) & * & / & * & / & * & * & & / & * & 5 \\
\hline Rashid et al. (2013) & * & I & * & l & I & * & & $\star \star$ & * & 6 \\
\hline Hosking et al. (2013) & * & * & * & * & * & * & & / & * & 7 \\
\hline Murakami et al. (2013) & * & * & * & * & * & * & & I & * & 7 \\
\hline Jiang et al. (2014) & * & / & * & $\star$ & $\star$ & * & & ** & * & 8 \\
\hline Tang et al. (2014b) & * & * & * & * & * & * & & I & * & 7 \\
\hline Stojanovic et al. (2015) & * & l & l & * & l & * & & $\star \star$ & * & 6 \\
\hline Yang et al. (2015b) & * & $\star$ & * & * & * & * & & $\star \star$ & * & 9 \\
\hline Terroni et al. (2015) & * & $\star$ & $\star$ & * & * & $\star$ & & $\star \star$ & * & 9 \\
\hline Saxena et al. (2015) & * & I & * & * & * & * & & $\star \star$ & I & 7 \\
\hline Gu et al. (2015) & * & l & * & * & * & * & & $\star \star$ & * & 8 \\
\hline Koivunen et al. (2015) & * & $\star$ & * & I & * & * & & / & * & 7 \\
\hline Metoki et al. (2016) & * & / & $\star$ & * & * & * & & $\star \star$ & $\star$ & 8 \\
\hline Pavlovic et al. (2016) & * & * & * & * & * & * & & $\star \star$ & * & 9 \\
\hline Zhang et al. (2016) & * & / & $\star$ & * & * & * & & $\star *$ & * & 8 \\
\hline \multirow[t]{2}{*}{ Study } & \multicolumn{6}{|c|}{ 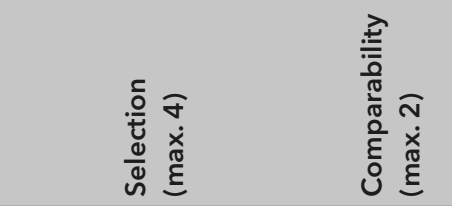 } & \multicolumn{3}{|c|}{ 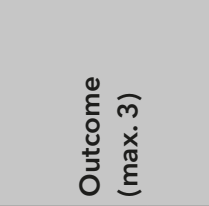 } & 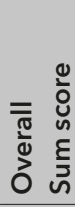 \\
\hline & S1 & S2 & S3 & S4 & C1 & $\mathrm{C} 2$ & 01 & $\mathrm{O} 2$ & O3 & \\
\hline \multicolumn{11}{|c|}{ Cohort studies (PSA) } \\
\hline Carota et al. (2005) & * & * & / & / & * & * & I & * & * & 6 \\
\hline Castellanos-Pinedo et al. (2011) & * & * & * & l & * & * & * & * & I & 7 \\
\hline Mikami et al. (2013) & * & / & * & * & * & * & * & $\star$ & * & 8 \\
\hline \multirow[t]{2}{*}{ Study } & \multicolumn{6}{|c|}{ 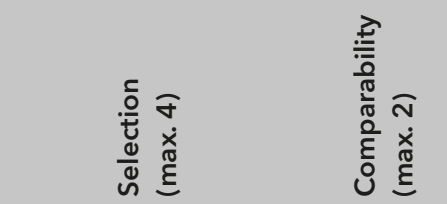 } & \multicolumn{3}{|c|}{ 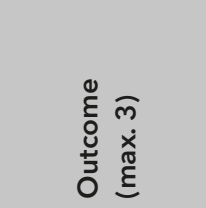 } & 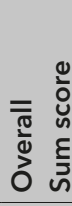 \\
\hline & S1 & S2 & S3 & S4 & C1 & $\mathrm{C} 2$ & 01 & $\mathrm{O} 2$ & O3 & \\
\hline \multicolumn{11}{|c|}{ Case-control studies (PSA) } \\
\hline Glodzik-Sobanska et al. (2005) & * & * & * & * & I & / & $\star$ & * & * & 7 \\
\hline $\begin{array}{l}\text { Brodaty et al. (2005) } \\
\text { Withall et al. (2011) }\end{array}$ & * & * & * & * & * & * & * & * & * & 9 \\
\hline Caeiro et al. (2012) & $\star$ & * & l & $\star$ & / & / & * & $\star$ & * & 6 \\
\hline Hollocks et al. (2015) & * & * & * & * & * & * & / & $\star$ & $\star$ & 8 \\
\hline
\end{tabular}




\begin{tabular}{|c|c|c|c|c|c|c|c|c|c|}
\hline \multirow[t]{2}{*}{ Study } & \multicolumn{4}{|c|}{ 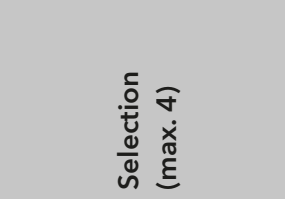 } & \multicolumn{2}{|c|}{ 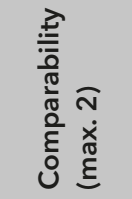 } & \multicolumn{2}{|c|}{ 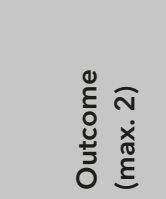 } & \multirow[t]{2}{*}{ 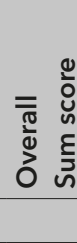 } \\
\hline & S1 & S2 & S3 & S4 & C1 & $\mathrm{C} 2$ & O1 & $\mathrm{O} 2$ & \\
\hline \multicolumn{10}{|c|}{ Cross-sectional studies (PSA) } \\
\hline Starkstein et al. (1993) & * & * & / & / & * & * & $\star \star$ & * & 7 \\
\hline Okada et al. (1997) & * & / & / & / & * & * & $\star \star$ & * & 6 \\
\hline Yamagata et al. (2004) & * & I & * & * & * & * & I & * & 6 \\
\hline Piamarta et al. (2004) & * & / & * & * & * & / & $\star \star$ & / & 6 \\
\hline Hama et al. (2007) & * & * & * & * & / & / & $\star \star$ & * & 7 \\
\hline Santa et al. (2008) & * & / & * & I & * & * & $\star \star$ & * & 7 \\
\hline Kang et al. (2008) & * & * & * & I & I & * & I & * & 5 \\
\hline Onoda et al. (2011) & * & / & * & * & * & * & $\star \star$ & * & 8 \\
\hline Murakami et al. (2013) & * & * & * & * & * & * & $\star \star$ & * & 9 \\
\hline Tang et al. (2013a) & * & * & * & * & * & * & ** & * & 9 \\
\hline Rochat et al. (2013) & * & I & * & I & * & I & $\star \star$ & * & 6 \\
\hline Yang et al. (2015c) & * & / & * & * & * & I & $\star \star$ & * & 7 \\
\hline Yang et al. (2015a) & * & I & * & * & * & * & ** & * & 8 \\
\hline Cosin et al. (2015) & * & * & * & * & * & * & $\star \star$ & * & 9 \\
\hline Mihalov et al. (2016) & * & / & * & I & * & * & $\star \star$ & * & 7 \\
\hline
\end{tabular}

Abbreviations: PSA, post-stroke apathy; PSD, post-stroke depression 
Supplementary Table 4. Characteristics of post-stroke depression studies

\begin{tabular}{|c|c|c|c|c|c|}
\hline Authors & $\begin{array}{c}\mathrm{N}, \\
\% \text { Fem }\end{array}$ & $\begin{array}{c}\text { Mean } \\
\text { age }\end{array}$ & $\%$ PSD* & Phase & Scale, criteria \\
\hline Robinson et al. (1983) & $103,38.8$ & 59.0 & 46.6 & $A$ & HRSD, PSE, SDS \\
\hline Robinson et al. (1984a) & $36,38.9$ & 59.0 & 44.4 & A & HRSD, PSE, SDS \\
\hline Robinson et al. (1984b) & $30,50.0$ & 59.4 & 36.7 & PA & HRSD, PSE, SDS \\
\hline Robinson et al. (1985a) & $103,43.7$ & 59.0 & NS & A, PA & HRSD, PSE, SDS \\
\hline Robinson et al. (1985b) & $61,44.4$ & 59.9 & NS & PA & HRSD, PSE, SDS \\
\hline Starkstein et al. (1988) & $26,26.9$ & 58.0 & NS & PA, C & HRSD, PSE, SDS \\
\hline Parikh et al. (1988) & $85,40.0$ & 63.0 & NS & A, PA, C & HRSD, PSE, SDS \\
\hline Eastwood et al. (1989) & $87,36.8$ & 63.3 & 54.0 & PA & HRSD, GDS, SDS, SADS, RDC \\
\hline Dam et al. (1989) & $92,37.0$ & 52.5 & 30.4 & PA & HRSD, BDI, RDC \\
\hline Starkstein et al. (1989) & $93,38.7$ & 62.2 & 30.1 & A & DSM-III, SDS, HDRS, PSE \\
\hline Sharpe et al. (1990) & $60,38.3$ & 71.0 & 18.3 & C & SCID, HADS \\
\hline House et al. (1990) & $128,54.7$ & 71.2 & 10.4 & PA, C & DSM-III, PSE, BDI $\geq 13$ \\
\hline Starkstein et al. (1991) & $56,41.1$ & 59.0 & 46.4 & $A$ & DSM-III, PSE, HDRS \\
\hline Stern and Bachman (1991) & $52,0.0$ & 65.8 & NS & C & VAMS, HRSD, DAC \\
\hline Morris et al. (1990) & $99,48.5$ & 70.8 & 32.3 & PA, C & CIDI (DSM-III) \\
\hline Morris et al. (1992) & $35,54.0$ & 68.4 & 40.0 & PA & DSM-III, MADRS \\
\hline Astrom et al. (1993) & $76,38.8$ & 72.0 & 25.0 & $A, P A, C$ & DSM-III \\
\hline Schwartz et al. (1993) & $91,0.0$ & 66.0 & 39.6 & PA & DSM-III, HDRS $\geq 18$ \\
\hline Herrmann et al. (1993) & $42,38.1$ & 62.0 & NS & A & CDS, RDC \\
\hline Sharpe et al. (1994) & $60,38.3$ & NS & 18.3 & C & SCID \\
\hline Andersen et al. (1995) & $285,46.0$ & 69.0 & 10.9 & PA & $\mathrm{BDI}, \mathrm{HDRS} \geq 13$ \\
\hline Herrmann et al. (1995) & $47,34.0$ & 62.0 & 36.2 & PA & DSM-III-R, MADRS, CDS \\
\hline $\mathrm{Ng}$ et al. (1995) & $52,44.2$ & 60.0 & 55.8 & PA & DSM-III-R, HDRS \\
\hline González et al. (1995) & $130,53.8$ & 67.6 & 36.9 & PA & RDC, HAM-D, MADRS, BDI \\
\hline Morris et al. (1996b) & $41,56.1$ & 70.0 & 36.6 & PA & DSM-III-R, MADRS, CIDI \\
\hline Morris et al. (1996a) & $193,51.8$ & 61.9 & 24.4 & A & CES-D $\geq 16$ \\
\hline Angeleri et al. (1997) & $180,35.0$ & 63.3 & 50.0 & C & $\mid C D-10, B D I \geq 10$ \\
\hline Bendsen et al. (1997) & $128, N S$ & NS & 15.6 & PA & SCID, HAM-D \\
\hline Gainotti et al. (1997) & $126, \mathrm{NS}$ & NS & 39.7 & PA & DSM-III, HDRS $>18$, PSDRS \\
\hline Herrmann et al. (1998) & $150,50.9$ & 74.9 & 27.3 & PA, C & $M A D R S \geq 7, S D S \geq 50$ \\
\hline Kase et al. (1998) & $74,54.1$ & 78.7 & 37.9 & PA & CES-D $\geq 16$ \\
\hline Huwel et al. (1998) & $102, \mathrm{NS}$ & 69.8 & 54.9 & A, PA & $M A D R S \geq 7, S D S$ \\
\hline Pohjasvaara et al. (1998) & $277, \mathrm{NS}$ & NS & 40.1 & PA & PSE, MADRS $\geq 6$ \\
\hline Vataja et al. (2001) & $275,48.7$ & 70.7 & 39.6 & PA & PSE, MADRS \\
\hline Vataja et al. (2004) & $70,50.0$ & 70.3 & 37.1 & PA & DSM-III-R, ICD-10 \\
\hline MacHale et al. (1998) & $55,48.0$ & 60.0 & 29.1 & PA & SADS, DSM-IV, HADS \\
\hline Gainotti et al. (1999) & $58,41.4$ & 61.9 & 29.3 & $A, P A$ & DSM-III, HAM-D > 17, PSDRS \\
\hline Gainotti et al. (2001) & $64,43.8$ & 61.9 & NS & PA & DSM-III, HDRS \\
\hline Paolucci et al. (1999) & $470,51.9$ & NS & 27.4 & PA & HDRS $\geq 18$, VADS \\
\hline Paradiso et al. (1999) & $141,40.4$ & 59.2 & 38.3 & A & DSM-IV, HAM-D, PSE \\
\hline
\end{tabular}




\begin{tabular}{|c|c|c|c|c|c|c|c|}
\hline \multirow{2}{*}{ First-ever } & \multirow{2}{*}{$\begin{array}{l}\text { Design, } \\
\text { PT source }\end{array}$} & \multirow{2}{*}{$\begin{array}{l}\text { Imaging } \\
\text { method }\end{array}$} & \multicolumn{5}{|c|}{ Imaging markers } \\
\hline & & & LAT & LOC & TP & LV & Other \\
\hline Y & $\mathrm{CS}, \mathrm{H} / \mathrm{R}$ & CT & $x$ & $x$ & - & $x$ & DIST FP \\
\hline Y & $\mathrm{CS}, \mathrm{H} / \mathrm{R}$ & CT & $x$ & $x$ & - & $x$ & DIST FP \\
\hline$Y$ & $\mathrm{P}, \mathrm{H} / \mathrm{R}$ & $\mathrm{CT}$ & $x$ & $x$ & - & $x$ & DIST FP \\
\hline Y & $\mathrm{CS}, \mathrm{H} / \mathrm{R}$ & $\mathrm{CT}$ & $x$ & $x$ & - & $x$ & DIST FP \\
\hline Y & $\mathrm{P}, \mathrm{H} / \mathrm{R}$ & $\mathrm{CT}$ & $x$ & $x$ & - & $x$ & DIST FP \\
\hline Y & CC, H/R & CT & - & - & - & - & ATR \\
\hline $\mathrm{N}$ & $\mathrm{P}, \mathrm{H}$ & CT & $x$ & - & $x$ & - & DIST FP \\
\hline $\mathrm{N}$ & $C C, R$ & CT & $x$ & - & - & $x$ & DIST FP \\
\hline $\mathrm{N}$ & $\mathrm{CS}, \mathrm{H}$ & CT & $x$ & $x$ & - & $x$ & - \\
\hline $\mathrm{N}$ & $\mathrm{CS}, \mathrm{H}$ & CT & - & $x$ & $x$ & $x$ & DIST FP \\
\hline$Y$ & $C S, C$ & CT & $x$ & - & $x$ & $x$ & DIST FP \\
\hline Y & $P, C$ & CT & $x$ & $x$ & - & $x$ & DIST FP \\
\hline $\mathrm{N}$ & $\mathrm{P}, \mathrm{H}$ & CT & $x$ & $x$ & - & - & DIST FP \\
\hline$Y$ & $C S, R$ & CT & $x$ & $x$ & - & - & - \\
\hline NS & $\mathrm{P}, \mathrm{H}$ & $\mathrm{CT}$ & $x$ & - & - & - & - \\
\hline Y & $\mathrm{CS}, \mathrm{H}$ & CT & $x$ & - & $x$ & $x$ & DIST FP \\
\hline $\mathrm{N}$ & $\mathrm{P}, \mathrm{H}$ & CT & - & $x$ & - & $x$ & DIST FP, ATR \\
\hline $\mathrm{N}$ & $C S, R$ & CT & $x$ & $x$ & - & $x$ & ATR \\
\hline $\mathrm{N}$ & CS, C & CT & - & $x$ & - & $x$ & DIST FP \\
\hline Y & $\mathrm{CS}, \mathrm{C}$ & $\mathrm{CT}$ & - & - & - & $x$ & - \\
\hline $\mathrm{N}$ & $\mathrm{P}, \mathrm{H}$ & CT & $x$ & $x$ & - & - & - \\
\hline Y & $\mathrm{CS}, \mathrm{H}$ & $\mathrm{CT}$ & $x$ & $x$ & - & $x$ & DIST FP, ATR \\
\hline $\mathrm{N}$ & $C S, R$ & CT & $x$ & - & $x$ & - & - \\
\hline NS & CS, R & CT & $x$ & $x$ & - & - & - \\
\hline$Y$ & $\mathrm{CS}, \mathrm{H}$ & CT & $x$ & $x$ & $x$ & $x$ & - \\
\hline$Y$ & $\mathrm{CS}, \mathrm{H}$ & $\mathrm{CT}$ & $x$ & $x$ & - & $x$ & DIST FP \\
\hline $\mathrm{N}$ & $\mathrm{CS}, \mathrm{H}$ & $\mathrm{CT}$ & $x$ & - & - & - & - \\
\hline $\mathrm{N}$ & $C S, R$ & CT & $x$ & - & - & - & NUM, ATR \\
\hline $\mathrm{N}$ & $\mathrm{CS}, \mathrm{H} / \mathrm{R}$ & CT & $x$ & $x$ & - & - & - \\
\hline N & $\mathrm{P}, \mathrm{H}$ & CT & $x$ & - & - & $x$ & - \\
\hline NS & CC, C & $\mathrm{CT}$ & $x$ & $x$ & - & - & - \\
\hline Y & $\mathrm{P}, \mathrm{H}$ & CT, NMR & $x$ & $x$ & - & - & - \\
\hline $\mathrm{N}$ & $\mathrm{CS}, \mathrm{H}$ & MRI & $x$ & $x$ & - & - & - \\
\hline $\mathrm{N}$ & $\mathrm{CS}, \mathrm{H}$ & MRI & $x$ & $x$ & - & $x$ & NUM, WMH, ATR \\
\hline $\mathrm{N}$ & $\mathrm{CS}, \mathrm{H}$ & MRI & $x$ & $x$ & - & $x$ & NUM, WMH, ATR \\
\hline $\mathrm{N}$ & $\mathrm{CS}, \mathrm{H}$ & $\mathrm{CT}$ & $x$ & $x$ & - & $x$ & DIST FP \\
\hline Y & $\mathrm{CS}, \mathrm{H} / \mathrm{R}$ & $\mathrm{CT}, \mathrm{MRI}$ & $x$ & $x$ & - & - & - \\
\hline Y & $\mathrm{CS}, \mathrm{H} / \mathrm{R}$ & CT & $x$ & - & - & - & - \\
\hline$Y$ & $\mathrm{CS}, \mathrm{H} / \mathrm{R}$ & $\mathrm{CT}, \mathrm{MRI}$ & $x$ & $x$ & - & - & - \\
\hline $\mathrm{N}$ & $\mathrm{CS}, \mathrm{H}$ & $\mathrm{CT}$ & $x$ & $x$ & $x$ & $X$ & DIST FP \\
\hline
\end{tabular}




\begin{tabular}{|c|c|c|c|c|c|}
\hline Authors & $\begin{array}{c}\mathrm{N}, \\
\% \text { Fem }\end{array}$ & $\begin{array}{c}\text { Mean } \\
\text { age }\end{array}$ & $\%$ PSD* & Phase & Scale, criteria \\
\hline Shimoda et al. (1999) & $60,43.4$ & 59.1 & 51.7 & $A$ & DSM-IV, HDRS, PSE \\
\hline Kim et al. (2000) & $148,36.5$ & 62.0 & 18.2 & PA & DSM-IV, BDI > 13 \\
\hline Hosking et al. (2000) & $79,54.0$ & 74.0 & 39.2 & PA & GDS $>9$ \\
\hline Singh et al. (2000) & $81,38.0$ & 70.0 & 35.8 & PA & $\mathrm{SDS} \geq 50, \mathrm{MADRS} \geq 7$ \\
\hline Berg et al. (2001) & $100,32.0$ & 55.2 & 27.0 & $A$ & $\mathrm{BDI} \geq 10, \mathrm{HDRS}, \mathrm{DSM}-\mathrm{II}-\mathrm{R}$ \\
\hline Rao et al. (2001) & $25, \mathrm{NS}$ & NS & 56.0 & C & HDRS, GDS, DSM-IV \\
\hline Spalletta et al. (2002) & $153,51.6$ & 66.3 & 57.5 & PA & SCID, HDRS \\
\hline Spalletta et al. (2005) & $200,58.0$ & 65.6 & 56.0 & PA & SCID-P, HAM-D, BDI \\
\hline Desmond et al. (2003) & $421,52.0$ & 70.8 & 11.2 & PA & $\operatorname{HDRS}(\mathrm{SIGH}-\mathrm{D})>11$ \\
\hline Berg et al. (2003) & $89,32.0$ & 55.2 & 27.0 & A, PA, C & DSM-III, HRSD, BDI > 9 \\
\hline Cassidy et al. (2004) & $50,42.0$ & 51.4 & 20.0 & PA & DSM-IV, CES-D $\geq 16$, HRSD $>7$ \\
\hline Verdelho et al. (2004) & $108,52.0$ & 75.0 & 42.6 & PA, C & MADRS $\geq 7$, CAMDEX \\
\hline Piamarta et al. (2004) & $33,39.4$ & 71.6 & 57.6 & A & PSDRS \\
\hline Aybek et al. (2005) & $254,46.5$ & 64.8 & 20.1 & A & $\mathrm{HDRS} \geq 8, \mathrm{EBI} \geq 1$ \\
\hline Nys et al. (2005) & $126,53.2$ & 62.3 & 52.4 & A & MADRS $\geq 8$ \\
\hline Hsieh and Kao (2005) & $207,40.0$ & 64.2 & 34.3 & A & HDRS $>10$ \\
\hline Tang et al. (2005) & $189,39.2$ & 68.2 & 14.6 & PA & SCID \\
\hline Kadojić et al. (2005) & $50,42.0$ & 65.1 & 72.0 & PA & $\mathrm{CCEI} \geq 4.9$ \\
\hline Paolucci et al. (2006) & $1064,40.1$ & 67.2 & 36.0 & $\mathrm{C}$ & DSM-IV, BDI $\geq 10$ \\
\hline Provinciali et al. (2008) & $731, N S$ & NS & 36.1 & C & MADRS, VAMS \\
\hline Glodzik et al. (2006) & $26,57.7$ & 63.4 & 30.8 & A, PA & DSM-IV, HDRS $\geq 13$ \\
\hline Aben et al. (2006) & $189,47.0$ & 68.5 & 21.7 & PA, C & SCID, HAM-D \\
\hline Leentjens et al. (2006) & $165,46.1$ & 68.1 & 23.0 & PA, C & SCID, HAM-D \\
\hline Bour et al. (2010) & $190,46.8$ & 68.6 & 18.8 & PA, C & SCID, HAM-D \\
\hline Caeiro et al. (2006) & $178,40.0$ & 56.8 & 46.1 & A & DSM-IV, MADRS $\geq 7$, PSDRS \\
\hline Wichowicz et al. (2006) & $60,28.3$ & 58.8 & 38.3 & PA & ICD-10 \\
\hline Barker-Collo (2007) & $57,10.2$ & 51.7 & 22.8 & PA & $\mathrm{BDI}-\mathrm{II}>19$ \\
\hline Hama et al. (2007) & $243,33.3$ & 65.2 & 51.9 & PA & $S D S \geq 45$ \\
\hline Brodaty et al. (2007) & $158,43.0$ & 72.1 & 12.0 & $\mathrm{PA}, \mathrm{C}$ & SCID \\
\hline Withall et al. (2011) & $106,39.6$ & 72.1 & 10.4 & PA & SCID \\
\hline Xu et al. (2008) & $38,26.3$ & 66.0 & All & A, PA & DSM-IV, HAM-D $\geq 7$ \\
\hline Fuentes et al. (2009) & $59,30.5$ & 65.3 & 28.8 & PA & $\mathrm{HDRS} \geq 8$ \\
\hline Snaphaan et al. (2009) & $283,45.0$ & 65.8 & 14.8 & PA & HADS $>8$ \\
\hline Iranmanesh et al. (2009) & $200,55.0$ & 61.2 & 32.0 & PA & DSM-IV, BDI \\
\hline Oladiji et al. (2009) & $51,39.2$ & 52.5 & 25.5 & C & DASS-21 $>9$ \\
\hline Chen et al. (2009) & $127,25.2$ & 64.4 & 51.2 & PA & $\mathrm{GDS} \geq 7$ \\
\hline Nishiyama et al. (2010) & $134,38.8$ & 67.3 & 34.3 & PA & $\mathrm{SDS} \geq 40$ \\
\hline Chau et al. (2010) & $210,41.0$ & 71.7 & 35.7 & PA & GDS 30 -item $>10$ \\
\hline Mok et al. (2010) & $77,41.6$ & 75.3 & 18.2 & PA & $\mathrm{NPI} \geq 1$ symptom \\
\hline Fu et al. (2010) & $45,40.0$ & 74.6 & 20.0 & PA & HADS-D $>7$ \\
\hline Huang et al. (2010) & $30,20.0$ & 70.0 & All & A & DSM-IV, HAM-D \\
\hline
\end{tabular}




\begin{tabular}{|c|c|c|c|c|c|c|c|}
\hline \multirow{2}{*}{ First-ever } & \multirow{2}{*}{$\begin{array}{l}\text { Design, } \\
\text { PT source }\end{array}$} & \multirow{2}{*}{$\begin{array}{l}\text { Imaging } \\
\text { method }\end{array}$} & \multicolumn{5}{|c|}{ Imaging markers } \\
\hline & & & LAT & LOC & TP & LV & Other \\
\hline$Y$ & $\mathrm{P}, \mathrm{H}$ & $\mathrm{CT}$ & $x$ & $x$ & - & $x$ & DIST FP \\
\hline$Y$ & $\mathrm{CS}, \mathrm{H}$ & $\mathrm{CT}, \mathrm{MRI}$ & $x$ & $x$ & $x$ & $x$ & WMH \\
\hline $\mathrm{N}$ & $\mathrm{CS}, \mathrm{H}$ & $\mathrm{CT}$ & $x$ & $x$ & - & - & - \\
\hline $\mathrm{N}$ & $\mathrm{P}, \mathrm{H}$ & CT & $x$ & $x$ & - & $x$ & DIST FP \\
\hline$Y$ & $\mathrm{CS}, \mathrm{H}$ & $\mathrm{CT}, \mathrm{MRI}$ & $x$ & - & - & - & - \\
\hline$Y$ & $\mathrm{CS} / \mathrm{CC}, \mathrm{C}$ & CT & - & $x$ & - & - & - \\
\hline $\mathrm{Y}$ & $\mathrm{CS}, \mathrm{H}$ & $\mathrm{CT}, \mathrm{MRI}$ & $x$ & $x$ & $x$ & $x$ & - \\
\hline Y & $\mathrm{CS}, \mathrm{H}$ & $\mathrm{CT}, \mathrm{MRI}$ & - & - & - & - & - \\
\hline $\mathrm{N}$ & $\mathrm{CC}, \mathrm{H}$ & $\mathrm{CT}, \mathrm{MRI}$ & $x$ & $x$ & - & - & - \\
\hline $\mathrm{N}$ & $\mathrm{P}, \mathrm{H}$ & NS & $x$ & - & - & - & - \\
\hline Y & $P, R$ & $\mathrm{CT}, \mathrm{MRI}$ & $x$ & - & - & - & - \\
\hline$N$ & $\mathrm{P}, \mathrm{H}$ & $\mathrm{CT}, \mathrm{MRI}$ & - & $x$ & $x$ & - & WMH, SI, ATR \\
\hline$Y$ & $\mathrm{CS}, \mathrm{H}$ & CT, MRI & $x$ & $x$ & - & - & - \\
\hline$Y$ & $\mathrm{CS}, \mathrm{H}$ & CT, MRI & - & $x$ & $x$ & $x$ & DIST FP \\
\hline$N$ & $\mathrm{CS} / \mathrm{CC}, \mathrm{H}$ & $\mathrm{CT}, \mathrm{MRI}$ & $x$ & $x$ & $x$ & $x$ & WMH, SI \\
\hline Y & $\mathrm{CS}, \mathrm{H}$ & CT, MRI & $x$ & - & - & - & - \\
\hline $\mathrm{N}$ & $\mathrm{CS}, \mathrm{H}$ & $\mathrm{CT}$ & $x$ & $x$ & - & - & DIST FP, NUM \\
\hline$N$ & $\mathrm{CS}, \mathrm{H}$ & $\mathrm{CT}$ & $x$ & - & - & - & - \\
\hline $\mathrm{N}$ & $\mathrm{P}, \mathrm{H}$ & $\mathrm{CT}, \mathrm{MRI}$ & $x$ & $x$ & $x$ & - & - \\
\hline Y & $\mathrm{P}, \mathrm{H}$ & $C T, M R I$ & - & - & - & - & - \\
\hline Y & $\mathrm{P} / \mathrm{CC}, \mathrm{H}$ & MRI, MRS & $x$ & $x$ & - & - & METAB \\
\hline$Y$ & $\mathrm{P}, \mathrm{H}$ & $\mathrm{CT}, \mathrm{MRI}$ & $x$ & $x$ & - & $x$ & WMH, SI \\
\hline Y & $\mathrm{P}, \mathrm{H}$ & $\mathrm{CT}, \mathrm{MRI}$ & $x$ & $x$ & - & - & WMH, SI \\
\hline Y & $\mathrm{P}, \mathrm{H}$ & $\mathrm{CT}, \mathrm{MRI}$ & $x$ & $x$ & - & - & - \\
\hline $\mathrm{N}$ & $\mathrm{CS} / \mathrm{CC}, \mathrm{H}$ & $\mathrm{CT}, \mathrm{MRI}$ & $x$ & $x$ & $x$ & - & - \\
\hline $\mathrm{N}$ & $\mathrm{CS}, \mathrm{H}$ & SPECT & $x$ & $x$ & - & - & $\mathrm{rCBF}$ \\
\hline$N$ & $\mathrm{CS}, \mathrm{H}$ & CT & $x$ & - & - & - & - \\
\hline $\mathrm{N}$ & $\mathrm{CS}, \mathrm{H}$ & $\mathrm{CT}$ & - & $x$ & - & $x$ & - \\
\hline $\mathrm{N}$ & $\mathrm{P} / \mathrm{CC}, \mathrm{H}$ & $\mathrm{CT}, \mathrm{MRI}$ & $x$ & - & - & - & NUM, WMH, ATR \\
\hline$N$ & $\mathrm{CS} / \mathrm{CC}, \mathrm{H}$ & CT, MRI & - & - & - & - & NUM, WMH, ATR \\
\hline $\mathrm{N}$ & $\mathrm{CC}, \mathrm{H}$ & MRS & - & - & - & - & METAB \\
\hline $\mathrm{N}$ & $\mathrm{P}, \mathrm{H}$ & $\mathrm{CT}$ & $x$ & - & - & - & - \\
\hline $\mathrm{N}$ & $\mathrm{CS}, \mathrm{H}$ & $\mathrm{CT}, \mathrm{MRI}$ & $x$ & $x$ & - & - & WMH, ATR \\
\hline Y & $\mathrm{P}, \mathrm{H}$ & MRI & - & $x$ & - & - & - \\
\hline $\mathrm{N}$ & $\mathrm{CS}, \mathrm{H}$ & NS & $x$ & - & - & - & - \\
\hline $\mathrm{N}$ & $\mathrm{CS}, \mathrm{H}$ & MRI & - & - & $x$ & $x$ & NUM, WMH \\
\hline$Y$ & $\mathrm{CS}, \mathrm{H}$ & $\mathrm{CT}, \mathrm{MRI}$ & - & $x$ & - & - & - \\
\hline $\mathrm{N}$ & $C S, R$ & $\mathrm{CT}$ & $x$ & - & $x$ & - & NUM \\
\hline$N$ & $\mathrm{CS}, \mathrm{H}$ & MRI & - & - & - & $x$ & WMH, CMB, ATR \\
\hline N & $\mathrm{CS}, \mathrm{H}$ & MRI & - & $x$ & - & - & NUM, WMH, CMB, ATR \\
\hline Y & $\mathrm{CS} / \mathrm{CC}, \mathrm{H}$ & MRI, MRS & - & - & - & - & METAB \\
\hline
\end{tabular}




\begin{tabular}{|c|c|c|c|c|c|}
\hline Authors & $\begin{array}{c}\mathrm{N}, \\
\% \mathrm{Fem}\end{array}$ & $\begin{array}{c}\text { Mean } \\
\text { age }\end{array}$ & \% PSD* & Phase & Scale, criteria \\
\hline Sienkiewicz et al. (2010) & $242,44.2$ & 65.5 & 33.9 & PA & GDS 15-item > 5 \\
\hline Williamson et al. (2010) & $108,49.1$ & 65.0 & NS & PA & $\mathrm{CMDI}, \mathrm{FrSBe}$ \\
\hline Nidhinandana et al. (2010) & $101,31.7$ & 60.7 & 46.5 & $\mathrm{C}$ & GDS (Thai) $>12$ \\
\hline Srivastava et al. (2010) & $51,19.6$ & 46.1 & 35.3 & C & HDRS, ICD-10 \\
\hline Chatterjee et al. (2010) & $127,43.7$ & 70.4 & 31.5 & C & DSM-IV, MADRS $>17$ \\
\hline Tang et al. (2010) & $994,53.9$ & 66.8 & 7.8 & PA & SCID, GDS \\
\hline Tang et al. (2011b) & $994,53.9$ & 66.8 & 7.8 & PA & SCID, GDS \\
\hline Tang et al. (2011c) & $591,39.1$ & 66.0 & 12.7 & PA & SCID, GDS \\
\hline Tang et al. (2013b) & $705,40.1$ & 66.3 & 12.1 & PA & SCID, GDS \\
\hline Tang et al. (2011a) & $235,39.2$ & 66.3 & 35.7 & PA & $\mathrm{GDS} \geq 7$ \\
\hline Terroni et al. (2011) & $55,47.1$ & 51.0 & 11.8 & A, PA & SCID, HAM-D \\
\hline Kim et al. (2011) & $133,46.6$ & 64.7 & 47.4 & A, PA & HADS-D $>7$ \\
\hline Effat et al. (2011) & $120,53.3$ & 56.7 & NS & PA & MINI, HAM-D \\
\hline Tennen et al. (2011) & $102,49.0$ & 70.8 & 37.3 & PA & CES-D $\geq 16$ \\
\hline Castellanos et al. (2011) & $89,48.3$ & $x$ & 42.7 & PA & $\mathrm{HAM}-\mathrm{D}>7, \mathrm{NPI}$ \\
\hline Marasco et al. (2011) & $54,27.8$ & 65.3 & 40.7 & A & PSDRS $\geq 9$ \\
\hline Altieri et al. (2012) & $105,34.3$ & 64.4 & 21.9 & PA & BDI-SF, SCID \\
\hline Zhang et al. (2012) & 163 , NS & $x$ & 23.9 & PA & DSM-IV, NPI, WHO-CIDI \\
\hline Choi-Kwon et al. (2012) & $508,38.8$ & 62.1 & 13.7 & A, PA & DSM-IV, BDI > 13 \\
\hline Wang et al. (2012) & $51,41.2$ & 61.1 & 21.6 & PA & $\mathrm{HAM}-\mathrm{D} \geq 14, \mathrm{SCID}$ \\
\hline Wongwandee et al. (2012) & $39,33.3$ & 59.7 & 28.2 & A & HDRS $>7$, DSM-III-R \\
\hline Chen et al. (2013) & $102,32.2$ & 72.6 & 30.4 & $A$ & HDRS $>10$ \\
\hline Ku et al. (2013) & $26,26.9$ & 60.8 & 11.5 & A & MINI, HADS \\
\hline Zhang et al. (2013) & $91,36.3$ & 60.0 & 27.5 & A, PA & $\mathrm{HAM}-\mathrm{D} \geq 7, \mathrm{WHO}-\mathrm{CIDI}$ \\
\hline Rajashekaran et al. (2013) & $62,21.0$ & 57.9 & 45.2 & PA & MINI, MADRS, BDI \\
\hline Taylor-Piliae et al. (2013) & $100,46.0$ & 70.0 & 35.0 & C & CES-D $\geq 16$ \\
\hline Rashid et al. (2013) & $60,28.3$ & 67.4 & NS & C & PSDRS \\
\hline Hosking and Marsh (2013) & $67,52.0$ & 74.0 & 32.8 & $\mathrm{C}$ & GDS $>9$ \\
\hline Murakami et al. (2013) & $149,34.9$ & 66.8 & 44.3 & PA & $\mathrm{HADS} \geq 8$ \\
\hline Shi et al. (2014) & $1067,35.2$ & 61.5 & 28.4 & $A, P A, C$ & DSM-IV, HRSD \\
\hline Yasuno et al. (2014) & $29,20.7$ & 68.7 & NS & PA & HAM-D, SDS \\
\hline De Ryck et al. (2014) & $94,42.8$ & 70.1 & 24.5 & PA, C & $\mathrm{CSD} \geq 8, \mathrm{MADRS}$ \\
\hline Jiang et al. (2014) & $392,30.4$ & 67.2 & 25.0 & PA & MDD: DSM-IV, MADRS $\geq 8$ \\
\hline Gozzi et al. (2014) & $55,43.6$ & 62.9 & 27.3 & PA & $\mathrm{HADS} \geq 11, \mathrm{MINI}$ \\
\hline Zhang et al. (2014) & $50,22.0$ & 58.2 & 52.0 & A & DSM-IV, HDRS $>7$ \\
\hline Brookes et al. (2014) & $101,31.0$ & 70.1 & 29.7 & C & GDS > 9 \\
\hline Tang et al. (2014b) & $229,29.3$ & 68.8 & 32.8 & PA & GDS $\geq 7$ \\
\hline Tang et al. (2014a) & $135,51.1$ & 65.7 & NS & PA, C & $\mathrm{GDS} \geq 7$ \\
\hline Stojanovic et al. (2015) & $118,50.0$ & $x$ & 11.0 & PA & $\mathrm{HRSD}>7$ \\
\hline Yang et al. (2015) & $116,34.5$ & 67.8 & 12.1 & A & DSM-IV, HAM-D $\geq 20$ \\
\hline Terroni et al. (2015) & $36,41.7$ & 51.5 & 19.4 & A & SCID \\
\hline
\end{tabular}




\begin{tabular}{|c|c|c|c|c|c|c|c|}
\hline \multirow{2}{*}{ First-ever } & \multirow{2}{*}{$\begin{array}{l}\text { Design, } \\
\text { PT source }\end{array}$} & \multirow{2}{*}{$\begin{array}{l}\text { Imaging } \\
\text { method }\end{array}$} & \multicolumn{5}{|c|}{ Imaging markers } \\
\hline & & & LAT & LOC & TP & LV & Other \\
\hline$Y$ & $\mathrm{CS}, \mathrm{H}$ & $\mathrm{CT}$ & $x$ & $x$ & $x$ & - & - \\
\hline $\mathrm{N}$ & $\mathrm{CS}, \mathrm{H}$ & MRI, DTI & - & - & - & - & FA values \\
\hline N & $\mathrm{CS}, \mathrm{H}$ & $\mathrm{CT}, \mathrm{MRI}$ & $x$ & - & - & - & - \\
\hline Y & $C S, R$ & NS & $x$ & - & $x$ & - & - \\
\hline $\mathrm{N}$ & $\mathrm{CS}, \mathrm{C}$ & CT & $x$ & $x$ & - & $x$ & NUM, WMH, ATR \\
\hline $\begin{array}{l}\mathrm{N} \\
\mathrm{N} \\
\mathrm{N} \\
\mathrm{N}\end{array}$ & $\begin{array}{l}\mathrm{CS}, \mathrm{H} \\
\mathrm{CS}, \mathrm{H} \\
\mathrm{CS}, \mathrm{H} \\
\mathrm{CS}, \mathrm{H}\end{array}$ & $\begin{array}{l}\text { MRI } \\
\text { MRI } \\
\text { MRI }\end{array}$ & $\begin{array}{l}X \\
- \\
-\end{array}$ & $\begin{array}{l}X \\
- \\
-\end{array}$ & $\begin{array}{l}- \\
- \\
-\end{array}$ & $\begin{array}{l}X \\
- \\
-\end{array}$ & $\begin{array}{c}\mathrm{CMB}, \mathrm{NUM}, \mathrm{WMH}, \\
\text { LACl, ATR } \\
-\end{array}$ \\
\hline $\mathrm{N}$ & $\mathrm{CS}, \mathrm{H}$ & MRI & - & $x$ & - & $x$ & $\mathrm{LACl}, \mathrm{WMH}, \mathrm{CMB}$ \\
\hline$Y$ & $\mathrm{P}, \mathrm{H}$ & MRI & $x$ & $x$ & - & $x$ & WMH \\
\hline $\mathrm{N}$ & $\mathrm{P}, \mathrm{H}$ & MRI & - & $x$ & - & - & WMH, CMB \\
\hline $\mathrm{N}$ & $\mathrm{CS}, \mathrm{H}$ & CT, MRI & - & $x$ & - & - & - \\
\hline $\mathrm{N}$ & $\mathrm{CS}, \mathrm{H} / \mathrm{R}$ & $\mathrm{CT}, \mathrm{MRI}$ & $x$ & - & - & - & - \\
\hline $\mathrm{N}$ & $\mathrm{P}, \mathrm{H}$ & $\mathrm{CT}, \mathrm{MRI}$ & $x$ & $x$ & - & - & - \\
\hline$Y$ & $\mathrm{CS}, \mathrm{H}$ & $\mathrm{CT}$ & $x$ & $x$ & - & - & - \\
\hline$N$ & $\mathrm{P}, \mathrm{H}$ & NS & $x$ & $x$ & - & - & WMH, ATR \\
\hline$N$ & $\mathrm{CS}, \mathrm{H}$ & MRI & $x$ & $x$ & - & $x$ & WMH \\
\hline$N$ & $\mathrm{P}, \mathrm{H}$ & MRI & $x$ & $x$ & - & - & WMC, CMB \\
\hline$Y$ & $\mathrm{CS} / \mathrm{CC}, \mathrm{H}$ & MRI, MRS & - & - & - & - & METAB \\
\hline$Y$ & $\mathrm{CS}, \mathrm{H}$ & CT, MRI & $x$ & $x$ & - & - & - \\
\hline $\mathrm{N}$ & $\mathrm{CS}, \mathrm{H}$ & $\mathrm{CT}, \mathrm{MRI}$ & $x$ & - & - & - & - \\
\hline$N$ & $\mathrm{P}, \mathrm{H}$ & MRI & $x$ & $x$ & - & $x$ & - \\
\hline$N$ & $P, H$ & $\mathrm{CT}, \mathrm{MRI}$ & $x$ & - & $x$ & - & - \\
\hline$Y$ & $\mathrm{CS}, \mathrm{H}$ & CT, MRI & $x$ & $x$ & - & - & - \\
\hline $\mathrm{N}$ & $C S, C$ & NS & $x$ & - & $x$ & - & - \\
\hline $\mathrm{N}$ & $\mathrm{CS}, \mathrm{H}$ & NS & $x$ & - & - & - & - \\
\hline $\mathrm{N}$ & $\mathrm{CS}, \mathrm{H}$ & CT & $x$ & $x$ & - & - & - \\
\hline$N$ & $\mathrm{CS}, \mathrm{H}$ & MRI & - & $x$ & - & - & - \\
\hline$Y$ & $\mathrm{P}, \mathrm{H}$ & CT, MRI & - & $x$ & - & - & - \\
\hline$N$ & $\mathrm{P}, \mathrm{H}$ & MRI, DTI & - & - & - & - & - \\
\hline$N$ & $\mathrm{P}, \mathrm{H}$ & CT, MRI & $x$ & - & $x$ & - & - \\
\hline$N$ & $\mathrm{CS}, \mathrm{H}$ & MRI & $x$ & $x$ & $x$ & - & NUM \\
\hline$Y$ & $\mathrm{P}, \mathrm{H}$ & MRI & $x$ & $x$ & - & $x$ & - \\
\hline$Y$ & $\mathrm{CC}, \mathrm{H}$ & MRI, fMRI & - & - & - & - & $\mathrm{FC}$ in ROIs \\
\hline$N$ & $\mathrm{CS}, \mathrm{H}$ & MRI, DTI & - & - & - & - & Median FA \\
\hline $\mathrm{N}$ & $\mathrm{CS}, \mathrm{H}$ & MRI & - & $x$ & - & $x$ & CMB, NUM, WMH \\
\hline $\mathrm{N}$ & $\mathrm{P}, \mathrm{H}$ & MRI & - & - & - & - & - \\
\hline $\mathrm{N}$ & $\mathrm{CS}, \mathrm{H}$ & CAT & - & $x$ & - & $x$ & - \\
\hline $\mathrm{N}$ & $\mathrm{CS}, \mathrm{H}$ & MRI, DTI & - & $x$ & - & - & FA maps \\
\hline$Y$ & $\mathrm{CS}, \mathrm{H}$ & MRI & $x$ & $x$ & - & $x$ & - \\
\hline
\end{tabular}




\begin{tabular}{|l|c|c|c|c|c|}
\hline Authors & $\begin{array}{c}\text { N, } \\
\% \text { Fem }\end{array}$ & $\begin{array}{c}\text { Mean } \\
\text { age }\end{array}$ & $\%$ PSD* & Phase & Scale, criteria \\
\hline Hollocks et al. (2015) & $118,34.7$ & 68.9 & NS & PA & GDS (24-item) \\
\hline Saxena and Suman (2015) & $107,43.9$ & 59.1 & 57.0 & A & MADRS > 6 \\
\hline Gu et al. (2015) & $196,33.7$ & 61.2 & 28.6 & PA & DSM-IV, HAM-D \\
\hline Wichowicz et al. (2015) & $116,36.2$ & 61.4 & 22.9 & A, PA, C & ICD-10, HRSD \\
\hline Koivunen et al. (2015) & $130,51.5$ & 41.5 & 23.1 & C & BDI > 13, HADS \\
\hline Wei et al. (2016) & $368,29.1$ & 61.5 & 19.3 & A, PA & DSM-IV, BDI > 13 \\
\hline Chen et al. (2016) & $207,27.5$ & 60.8 & 41.1 & A, PA & HDRS $\geq 8$ \\
\hline Metoki et al. (2016) & $421,37.3$ & 72.1 & 16.9 & A & JSS-D $\geq 2.4$ \\
\hline Pavlovic et al. (2016) & $294,46.3$ & 62.3 & 39.8 & C & DSM-IV, HDRS \\
\hline Zhang et al. (2016) & $251,27.9$ & 66.0 & 17.9 & A & HAM-D $\geq 7$ \\
\hline
\end{tabular}

*Percentage of participants with PSD at baseline measurement.

Note: Studies presented within the same row represent studies of the same cohort.

Abbreviations: A, acute; ATR, atrophy; C, chronic (phase); BDI, Beck Depression Inventory; BDI-II, Beck Depression Inventory Second Edition; BDI-SF, Beck Depression Inventory Short Form; C, community (patient source); CAMDEX, Cambridge Mental Disorders of the Elderly Examination; CC, case-control; CCEI, Crown-Crips Experiential Index; CDS, CSD Cornell Scale for Depression; CES-D, Center for Epidemiologic Studies Depression Scale; CIDI, Composite International Diagnostic Interview; CMB, cerebral microbleeds; CMDI, Chicago Multiscale Depression Inventory; CS, cross-sectional; CT, computed tomography; DAC, Depression Adjective Checklist; DASS-21, Depression Anxiety Stress Scale 21-item version; DIST FP, distance to frontal pole; DSM-III, Diagnostic and Statistical Manual of Mental Disorders Third Edition; DSM-III-R, Diagnostic and Statistical Manual of Mental Disorders Third Edition revised; DSM-IV, Diagnostic and Statistical Manual of Mental Disorders Fourth Edition; DTI, diffusion tensor imaging; EBI, Emotional Behavior Index; FA, fractional anisotropy; Fem, female; $\mathrm{fMRI}$, functional magnetic resonance imaging; FrSBe, Frontal Systems Behavior Scale; GDS, Geriatric Depression Scale; H, hospital; HADS, Hospital Anxiety and Depression Scale; HRSD, HDRS, HAM-D, Hamilton Rating Scale for depression; ICD-10, International Statistical Classification of Diseases and Related Health Problems 10th Revision; JSS-D, Japan Stroke Scale Depression Scale; LACl, lacunar infarcts; LAT, laterality; LOC, location; LV, lesion volume; MADRS, Montgomery-Åsberg Depression Rating Scale; METAB, metabolism; MINI, Mini International Neuropsychiatric Interview; MRI, magnetic resonance imaging, MRS, proton magnetic resonance spectroscopy; $\mathrm{N}$, number of participants; $\mathrm{N}$, no; NMR, nuclear magnetic resonance; NPI, Neuropsychiatric Inventory; NS, not specified; NUM, number of lesions; P, prospective; PA, post-acute; PSD, post-stroke depression; PSDRS, Post-Stroke Depression Rating Scale; PSE, Present State Examination; PT, patient; R, rehabilitation center; rCBF, regional cerebral blood flow; RDC, Research Diagnostic Criteria; ROIs, regions of interest; SADS, Schedule for Affective Disorders and Schizophrenia; SCID, Structured Clinical Interview for DSM Disorders; SCID-P, Structured Clinical Interview for DSM Disorders Patient Edition; SDS, Zung Self-rating Depression Scale; SI, silent infarcts; SIGH-D, Structured Interview Guide for the HDRS; SPECT, single-photon emission computed tomography; TP, type of stroke; VAMS, Visual Analogue Mood Scale; WHO-CIDI, World Health Organization Composite International Diagnostic Interview; WM, white matter; WMC, white matter changes; $\mathrm{WMH}$, white matter hyperintensities; $\mathrm{Y}$, yes. 
Imaging markers of PSD and PSA: a systematic review and meta-analysis

\begin{tabular}{|c|c|c|c|c|c|c|c|}
\hline \multirow{2}{*}{ First-ever } & \multirow{2}{*}{$\begin{array}{l}\text { Design, } \\
\text { PT source }\end{array}$} & \multirow{2}{*}{$\begin{array}{l}\text { Imaging } \\
\text { method }\end{array}$} & \multicolumn{5}{|c|}{ Imaging markers } \\
\hline & & & LAT & LOC & TP & LV & Other \\
\hline Y & $\mathrm{CS}, \mathrm{H}$ & MRI, DTI & - & - & - & - & FA, WM networks \\
\hline $\mathrm{N}$ & $\mathrm{CS}, \mathrm{H}$ & $\mathrm{CT}, \mathrm{MRI}$ & $x$ & $x$ & $x$ & $x$ & - \\
\hline $\mathrm{N}$ & $\mathrm{CS}, \mathrm{H}$ & $\mathrm{CT}, \mathrm{MRI}$ & - & $x$ & - & - & - \\
\hline$Y$ & $\mathrm{P}, \mathrm{H}$ & $\mathrm{CT}, \mathrm{MRI}$ & $x$ & $x$ & - & - & - \\
\hline Y & $\mathrm{CS}, \mathrm{H}$ & $C T, M R I$ & - & $x$ & - & $x$ & NUM \\
\hline $\mathrm{N}$ & $\mathrm{P}, \mathrm{H}$ & CT, MRI & $x$ & $x$ & - & - & WMH \\
\hline $\mathrm{N}$ & $\mathrm{P}, \mathrm{H}$ & MRI & $x$ & $x$ & - & $x$ & NUM, WMH, ATR \\
\hline NS & $\mathrm{CS}, \mathrm{H}$ & MRI & $x$ & $x$ & - & - & - \\
\hline Y & $\mathrm{CS}, \mathrm{H}$ & MRI & - & - & - & - & LACl, WMH \\
\hline $\mathrm{N}$ & $\mathrm{CS}, \mathrm{H}$ & $\mathrm{CT}, \mathrm{MRI}$ & $x$ & $x$ & - & - & - \\
\hline
\end{tabular}


Supplementary Table 5. Characteristics of post-stroke apathy studies

\begin{tabular}{|c|c|c|c|c|c|c|}
\hline Study & Year & $\mathrm{N}, \%$ Fem & $\begin{array}{c}\text { Mean } \\
\text { age }\end{array}$ & $\%$ PSA* $^{*}$ & Phase & Scale \\
\hline Starkstein et al. (1993) & 1993 & $80,46.3$ & 59.5 & 22.5 & A & $A S \geq 14$ \\
\hline Okada et al. (1997) & 1997 & $40,42.5$ & 71.4 & 50.0 & C & $A S \geq 16$ \\
\hline Yamagata et al. (2004) & 2004 & $29,72.4$ & 71.7 & 55.2 & PA & $A S \geq 16$ \\
\hline Piamarta et al. (2004) & 2004 & $33,39.4$ & 71.6 & 15.2 & A & PSDRS expanded $>4$ \\
\hline Carota et al. (2005) & 2005 & $273,46.9$ & 64.4 & 47.6 & A & $\mathrm{EBIF} \geq 0.5$ \\
\hline Glodzik et al. (2005) & 2005 & $31,48.4$ & 62.9 & 41.9 & A & $A S>16$ \\
\hline Brodaty et al. (2005) & 2005 & $135,39.3$ & 72.2 & 26.7 & PA & $A E S \geq 37$ \\
\hline Withall et al. (2011) & 2011 & $106,39.6$ & 72.1 & 25.5 & PA & $A E S \geq 37$ \\
\hline Hama et al. (2007) & 2007 & $243,33.3$ & 65.2 & 40.3 & PA & $A S \geq 16$ \\
\hline Santa et al. (2008) & 2008 & $67,43.3$ & 65.4 & 20.9 & PA & $A S>15$ \\
\hline Kang and Kim (2008) & 2008 & $100,42.0$ & 65.1 & 43.0 & A & Clinical observation \\
\hline Onoda et al. (2011) & 2011 & $102,44.1$ & 73.0 & 36.3 & A, PA & $A S \geq 16$ \\
\hline Castellanos et al. (2011) & 2011 & $89,48.3$ & 70.0 & 13.5 & PA & NPI \\
\hline Caeiro et al. (2012) & 2012 & $94,35.1$ & 55.7 & 38.3 & A & AES $-10 \geq 18$ \\
\hline Murakami et al. (2013) & 2013 & $149,34.9$ & 66.8 & 44.3 & PA & $A S \geq 16$ \\
\hline Mikami et al. (2013) & 2013 & $56,35.7$ & 63.9 & 41.1 & PA, C & AS, criteria Robert \\
\hline Tang et al. (2013a) & 2013 & $185,37.3$ & 65.2 & 10.8 & PA & $A E S \geq 37$ \\
\hline Rochat et al. (2013) & 2013 & $55, \mathrm{NS}$ & 56.4 & NS & PA, C & $\mathrm{Al}$ \\
\hline Yang et al. (2015c) & 2015 & $54,35.2$ & 67.4 & 57.4 & PA & AES, criteria Robert \\
\hline Yang et al. (2015a) & 2015 & $88,27.3$ & 68.2 & 38.6 & A, PA & AES, criteria Robert \\
\hline Cosin et al. (2015) & 2015 & $46,17.4$ & 58.0 & 21.7 & PA & $\mathrm{Al}>2, \mathrm{LARS}>21$ \\
\hline Hollocks et al. (2015) & 2015 & $118,34.7$ & 68.9 & NS & PA & GDS (6-items) \\
\hline Mihalov et al. (2016) & 2016 & $47,36.2$ & 67.7 & 23.4 & PA & $\mathrm{AS} \geq 14$ \\
\hline
\end{tabular}

Note: *Percentage of participants with PSA at baseline measurement.

Abbreviations: A, acute; AES, Apathy Evaluation Scale; AES-10, Apathy Evaluation Scale 10-item; AS, Apathy Scale; ATR, atrophy; C, chronic (phase); C, community (patient source); CC, case-control; CMB, cerebral microbleeds; CS, cross-sectional; $\mathrm{CT}$, computed tomography; DTI, diffusion tensor imaging; EBIF, Emotion Behavior Index Form; FA, fractional anisotropy; Fem, female; $\mathrm{H}$, hospital; LACl, lacunar infarcts; LARS, Lille Apathy Rating Scale; LAT, laterality; LOC, location; LV, lesion volume; METAB, metabolism; MRA, magnetic resonance angiography; MRI, magnetic resonance imaging; MRS, proton magnetic resonance spectroscopy; N, number of participants; N, no; NPI, Neuropsychiatric Inventory; NS, not specified; NUM, number of lesions; P, prospective; PA, post-acute; PSA, post-stroke apathy; PSDRS, Post-Stroke Depression Rating Scale; PT, patient; $R$, rehabilitation center; $r C B F$, regional cerebral blood flow; SPECT, single-photon emission computed tomography; TP, type of stroke; WM,

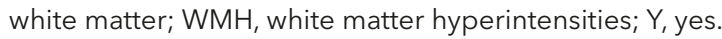




\begin{tabular}{|c|c|c|c|c|c|c|c|}
\hline \multirow{2}{*}{$\begin{array}{l}\text { First- } \\
\text { ever }\end{array}$} & \multirow{2}{*}{$\begin{array}{l}\text { Design, } \\
\text { PT source }\end{array}$} & \multirow{2}{*}{$\begin{array}{l}\text { Imaging } \\
\text { method }\end{array}$} & \multicolumn{5}{|c|}{ Imaging markers } \\
\hline & & & LAT & LOC & TP & LV & Other \\
\hline Y & $\mathrm{CS}, \mathrm{H}$ & CT & $x$ & $x$ & $x$ & $x$ & - \\
\hline $\mathrm{N}$ & $\mathrm{CS}, \mathrm{H}$ & CT, MRI & - & $x$ & - & - & $\mathrm{rCBF}$ \\
\hline $\mathrm{N}$ & $\mathrm{CS}, \mathrm{H}$ & MRI & $x$ & $x$ & - & $x$ & NUM \\
\hline$Y$ & $\mathrm{CS}, \mathrm{H}$ & CT, MRI & $x$ & $x$ & - & - & - \\
\hline Y & $\mathrm{P}, \mathrm{H}$ & $\mathrm{CT}, \mathrm{MRI}$ & $x$ & $x$ & - & - & - \\
\hline$Y$ & $\mathrm{CC}, \mathrm{H}$ & CT, MRI, MRS & $x$ & $x$ & - & - & METAB \\
\hline Y & $\mathrm{CC}, \mathrm{H}$ & $\mathrm{CT}, \mathrm{MRI}$ & $x$ & - & - & $x$ & NUM, ATR, WMH \\
\hline Y & $\mathrm{P}, \mathrm{H}$ & $\mathrm{CT}, \mathrm{MRI}$ & - & - & - & - & ATR, WMH \\
\hline $\mathrm{N}$ & $\mathrm{CS}, \mathrm{H}$ & CT & - & $x$ & - & $x$ & - \\
\hline Y & $C S, R$ & MRI & $x$ & $x$ & $x$ & - & - \\
\hline $\mathrm{N}$ & $\mathrm{CS}, \mathrm{H}$ & MRI, MRA & $x$ & $x$ & - & - & - \\
\hline $\mathrm{N}$ & $\mathrm{CS}, \mathrm{H}$ & MRI, SPECT & - & $x$ & - & - & $\mathrm{rCBF}$ \\
\hline $\mathrm{N}$ & $\mathrm{P}, \mathrm{H}$ & CT, MRI & $x$ & $x$ & - & - & - \\
\hline $\mathrm{N}$ & $\mathrm{CC}, \mathrm{H}$ & $\mathrm{CT}, \mathrm{MRI}$ & $x$ & $x$ & $x$ & - & - \\
\hline $\mathrm{N}$ & $\mathrm{CS}, \mathrm{H}$ & MRI & - & $x$ & - & - & - \\
\hline $\mathrm{N}$ & $\mathrm{P}, \mathrm{H} / \mathrm{C}$ & NS & - & - & $x$ & - & - \\
\hline $\mathrm{N}$ & $\mathrm{CS}, \mathrm{H}$ & MRI & - & $x$ & - & $x$ & NUM, WMH \\
\hline Y & $\mathrm{CS}, \mathrm{H}$ & $C T, M R I$ & - & $x$ & - & $x$ & - \\
\hline $\mathrm{N}$ & $\mathrm{CS}, \mathrm{H}$ & MRI, DTI & - & $x$ & - & - & FA maps \\
\hline $\mathrm{N}$ & $\mathrm{CS}, \mathrm{H}$ & MRI, DTI & - & - & - & $X$ & $\begin{array}{l}\text { WM integrity, } \\
\text { WM networks }\end{array}$ \\
\hline $\mathrm{N}$ & $\mathrm{CS}, \mathrm{H}$ & MRI & - & $x$ & - & - & $\mathrm{LACl}, \mathrm{WMH}, \mathrm{CMB}$ \\
\hline$Y$ & $\mathrm{CS}, \mathrm{H}$ & MRI, DTI & - & - & - & - & FA,WM networks \\
\hline $\mathrm{N}$ & $\mathrm{CS}, \mathrm{H}$ & CT, MRI & - & - & - & - & ATR \\
\hline
\end{tabular}



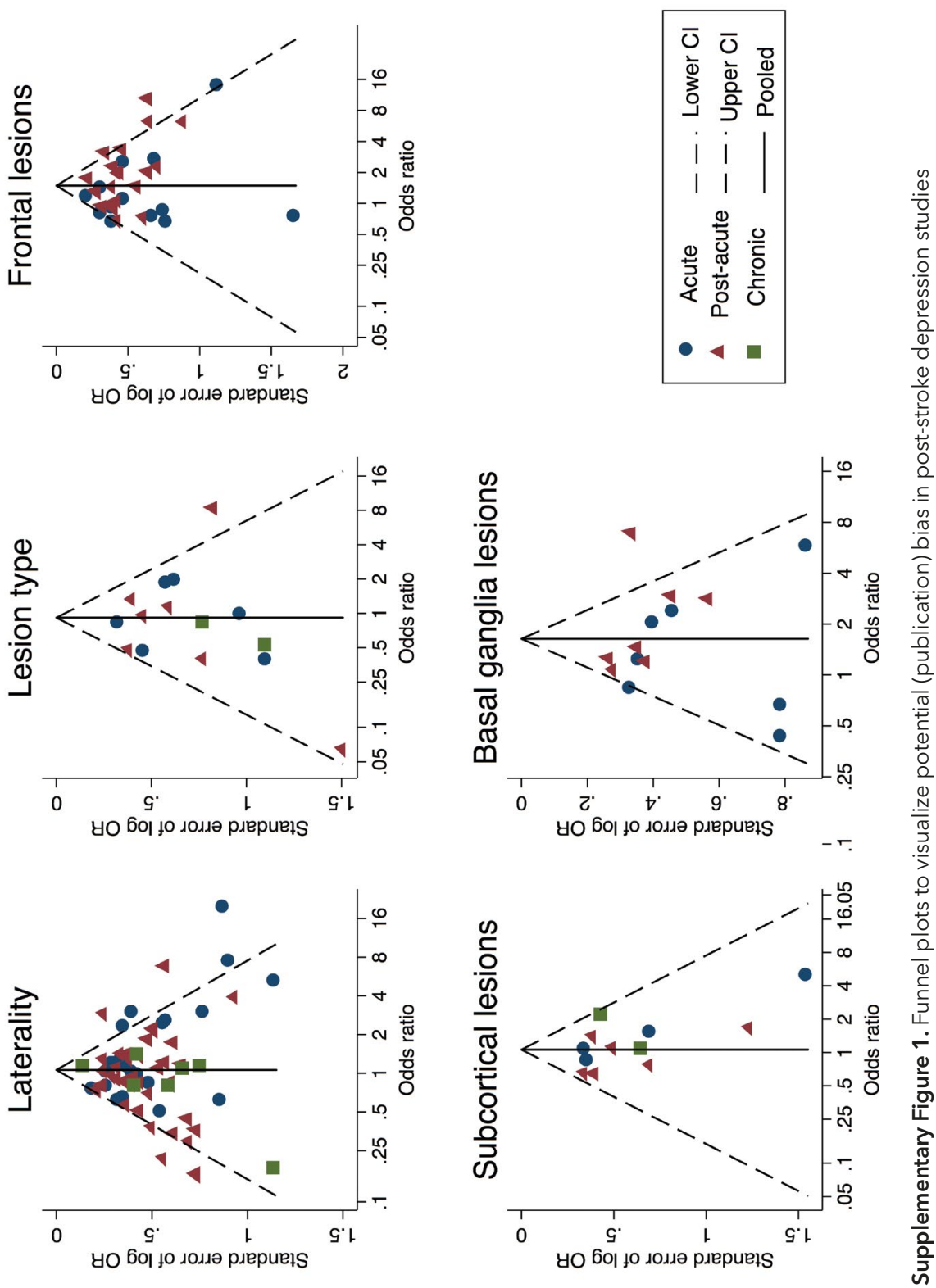

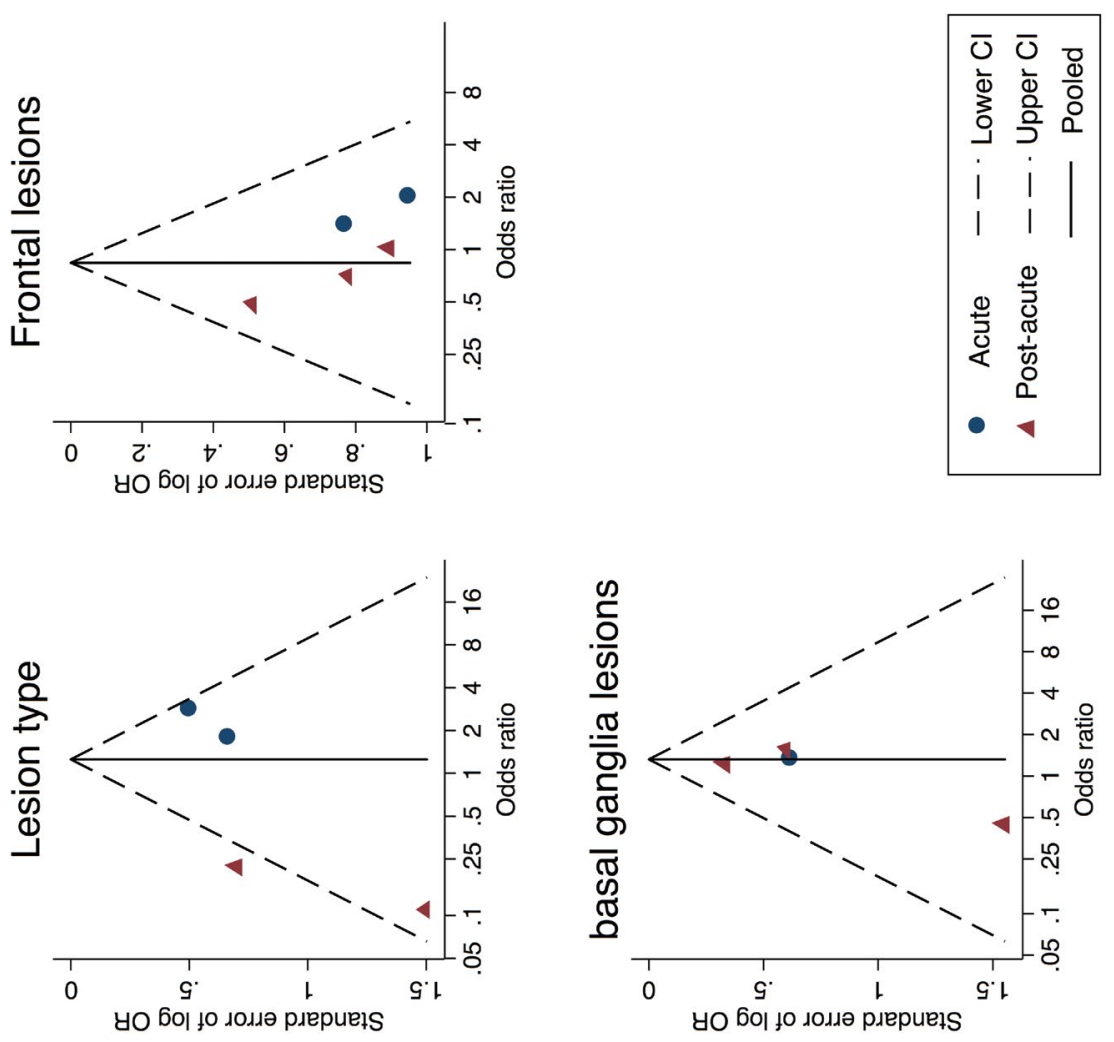

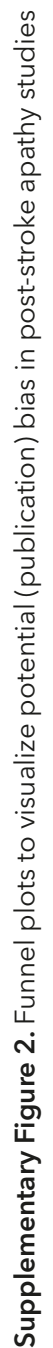
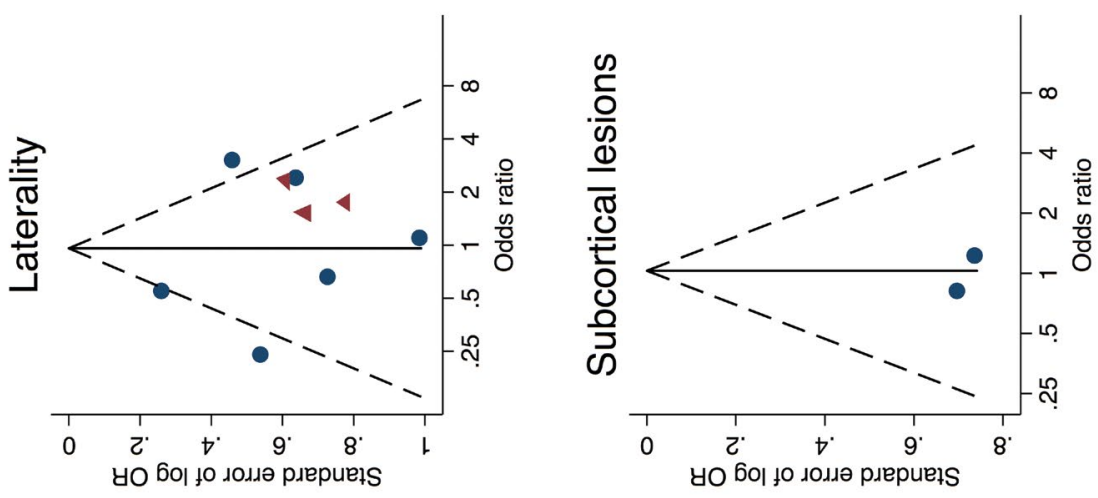


\section{CHAPTER 3}

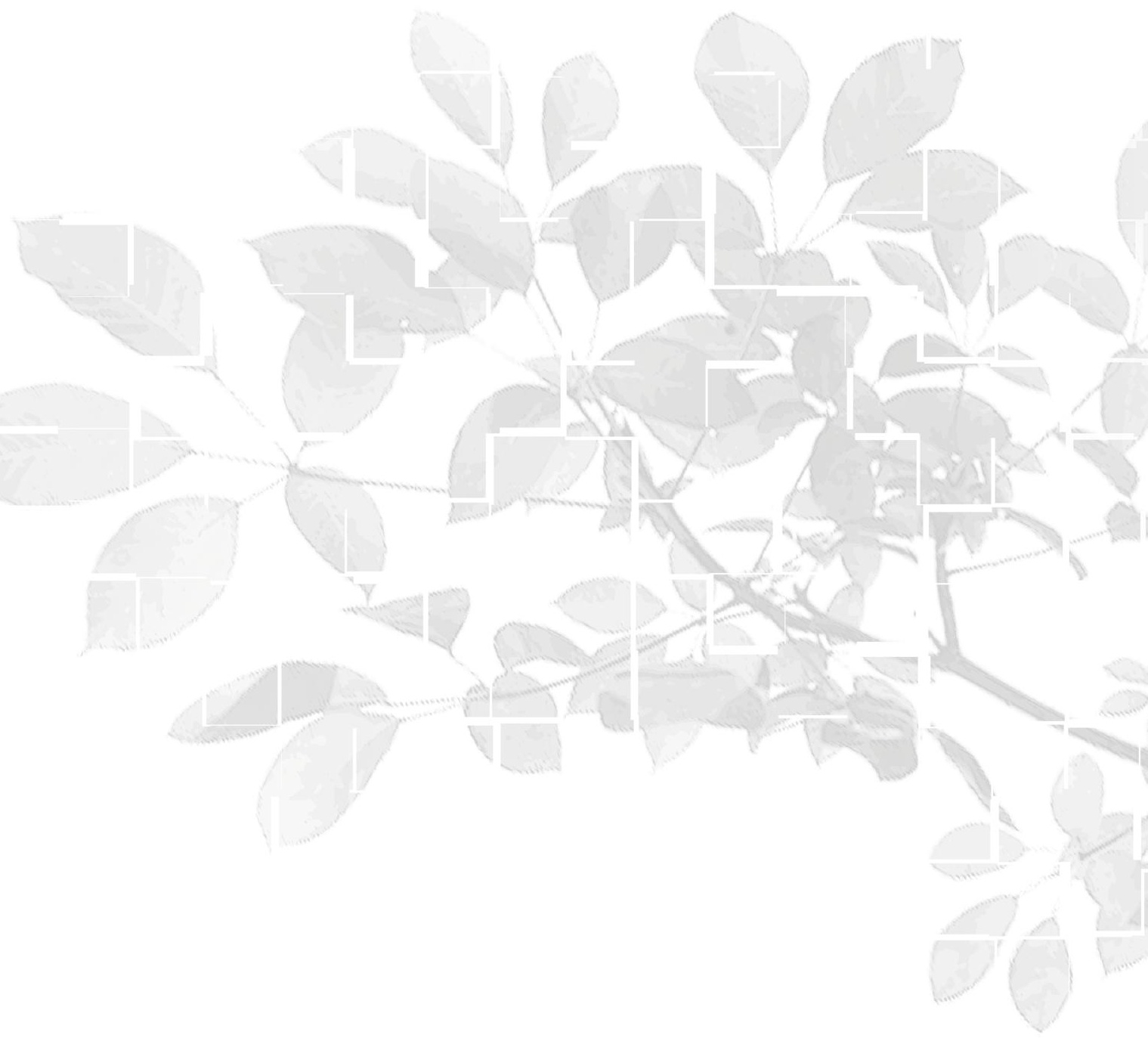




\section{THE COGNITION AND AFFECT AFTER STROKE - A PROSPECTIVE EVALUATION OF RISKS (CASPER) STUDY: RATIONALE AND DESIGN}

Elles Douven

Syenna HJ Schievink

Frans RJ Verhey

Robert J van Oostenbrugge

Pauline Aalten

Julie Staals

Sebastian Köhler 


\section{ABSTRACT}

Background: Cognitive impairment and neuropsychiatric syndromes, like depression and apathy, are frequent residual consequences of stroke. These have a large impact on quality of life and long-term prognosis. Several factors are involved in the development of these residual syndromes, although their exact role and their interrelationships remain still rather unclear. The Cognition and Affect after Stroke: a Prospective Evaluation of Risks (CASPER) study has been primarily designed to examine whether stroke-specific (e.g. lesion location, volume, type, severity), cerebrovascular and neurodegenerative (e.g. white matter changes, atrophy, microbleeds, perivascular spaces), inflammatory, endothelial, and (epi)genetic markers are associated with cognitive impairment, post-stroke depression, and post-stroke apathy, and whether they predict their course over 12 months. The secondary aims are to investigate how the above-mentioned markers interact with each other, and to determine if patients with apathy and depression after stroke differ in pathogenesis, course, and outcome (e.g. functional outcome, neurocognitive performance, quality of life).

Methods/design: CASPER is a 1-year prospective clinical cohort follow-up study in 250 stroke patients recruited at the neurological in- and outpatient services at Maastricht University Medical Center (MUMC+, Maastricht, the Netherlands), and Zuyderland Medical Center (Sittard and Heerlen, the Netherlands). At baseline (3 months post-stroke), a neuropsychological assessment, neuropsychiatric interview, blood sample, and brain magnetic resonance imaging (MRI) scan are conducted. Assessment of neuropsychiatric and neurocognitive status are repeated 6 and 12 months later.

Discussion: The CASPER study investigates stroke-specific, vascular, neurodegenerative, inflammatory, and genetic markers of the development of vascular cognitive impairment, depression, and apathy after stroke. This creates the possibility to study not only the contribution of these individual markers but also their joint contribution, which differentiates this study from earlier stroke cohorts who lacked long-term follow-up data, a large sample size, an extensive MRI protocol, and markers from the blood. The knowledge we derive from this study might help in identifying markers that are associated with, or can predict the onset, maintenance, and progression of vascular cognitive impairment, depression, and apathy after stroke, and could provide new insights into possibilities for treatment and rehabilitation that result in better functional outcome after stroke. 


\section{BACKGROUND}

Stroke is one of the leading causes of disability in the Western world. According to the Global Burden of Disease Study in 2010 the stroke incidence worldwide was around 17 million persons per year, with 33 million people being still alive after stroke, and about $70 \%$ of all stroke patients staying with residual symptoms. ${ }^{1}$ Stroke is the second most frequent cause of death, after coronary artery disease. ${ }^{2}$ As a result of the aging population, this number will probably increase steadily in the next decades. ${ }^{3}$ At the same time, case fatality rates are declining due to better acute treatment. Therefore, more and more individuals will need to learn how to deal with the residual disabilities and handicaps. ${ }^{1}$ Frequent impairments after stroke are cognitive impairments and neuropsychiatric syndromes, including depression and apathy, ${ }^{4,5}$ which have an impact on long-term prognosis (higher mortality and more disability), and quality of life (QoL) of stroke survivors. ${ }^{6,7}$

\section{VASCULAR COGNITIVE IMPAIRMENT}

Vascular cognitive impairment ( $\mathrm{VCl}$ ) describes the full spectrum from mild to severe cognitive impairment in people with cerebrovascular disease, including vascular mild cognitive impairment and vascular (or post-stroke) dementia (VaD). ${ }^{8}$ Risk factors of $\mathrm{VCl}$ after stroke include event-related factors such as infarct severity, lesion volume, age, low education, history of diabetes or atrial fibrillation, and number of recurrent strokes. ${ }^{9}$ The risk for post-stroke dementia is known to be highest within the first year, with an estimated incidence rate of $20-30 \%$, which is nine-times the general population's risk. ${ }^{9}$

It has been suggested that both vascular and neurodegenerative pathologies contribute to post-stroke VCl. ${ }^{10}$ Cognitive impairments can be the result of strategic infarcts, but (pre-existing) white matter hyperintensities (WMH) and atrophy of the medial temporal lobes can contribute too, and they probably interact with each other, though the nature of these interactions is not well understood. ${ }^{11}$ In addition, a higher risk for $\mathrm{VCl}$ has been reported in carriers of the Apolipoprotein $E(A p o E) \varepsilon 4$ allele, which is the major genetic risk factor for Alzheimer's disease (AD) dementia. ${ }^{12}$ Indeed, evidence accumulates that AD and VaD should not be regarded as mutually exclusive diagnoses, but rather as a continuum with pure $A D$ and $V a D$ at its extremes, and most people showing mixtures of both type of pathologies. ${ }^{13}$ While the dominating view is that vascular changes work as a catalyst of primary neurodegenerative changes in $A D$ it is largely unknown whether the opposite is true for $\mathrm{VCl}$, i.e. whether AD-related changes work as a catalyst for post-stroke dementia. 
Next to neurodegeneration, neuro- and vascular inflammation might contribute to $\mathrm{VCl}$ by partly mediating the pathophysiology underlying $\mathrm{VCl}$ as part of a final common pathway. ${ }^{14}$ Following stroke, increased auto-immune activity is a common response, and it has been shown that up-regulated inflammation markers like C-reactive protein (CRP), as a consequence of a prolonged auto-immune response, relate to poor prognosis. This includes a higher risk of additional future strokes and mortality, but also more severe VCl.15,16 Other enzymes, molecules, and ligands (myeloperoxidase, soluble intercellular adhesion molecule, soluble vascular cell adhesion molecule-1, soluble $E$ selectin, soluble $P$ selectin and CD40 ligand) are involved in vascular inflammation as part of an inflammatory response to stroke, and have also been found to be increased in AD. ${ }^{17-20}$ Also, upregulation of proinflammatory cytokines (interleukin-6 and tumor necrosis factor alpha) and proteins (high sensitivity CRP) are risk factors for both dementia ${ }^{21,22}$ and cardiovascular disease. ${ }^{23}$ It is unknown whether similar inflammation mechanisms are involved in cognitive deterioration in $\mathrm{VCl}$, and therefore, research is needed to investigate whether inflammation contributes to $\mathrm{VCl}$ independently or in joint action with other (e.g. neuro-degenerative) factors.

Finally, genetic factors are also suggested to play a role in the pathogenesis of $\mathrm{VCl}$, but in contrast to $A D$, evidence is still scarce. A genome-wide association study in $\mathrm{VaD}$ identified a novel genetic locus near the androgyn receptor, and this finding was replicated in two independent validation datasets. ${ }^{24}$ Polymorphisms and mutations on the genes coding for angiotensin-converting enzyme and methylenetetrahydrofolate reductase are risk factors for cardiovascular diseases and might be related to the development of $\mathrm{VaD}$, although this relationship is still controversial. ${ }^{25}$ For $A D$, several genetic risk loci are already identified next to ApoE, including clusterin (CLU), phosphatidylinositol binding clathrin assembly protein (PICALM), and encoding complement component [3b/4b] receptor 1 (CR1), ${ }^{26}$ but their effects are generally small (see http://www.alzgene.org). Whether they contribute to post-stroke dementia is not known.

\section{POST-STROKE DEPRESSION}

Depression is a common neuropsychiatric syndrome following stroke. According to a recent systematic review by Hackett et al., ${ }^{27}$ around one-third of the stroke survivors experiences depression in the first three months post-stroke, and studies with long-term follow-up have shown that it is often a chronic disorder, with a remitting-relapsing pattern. ${ }^{28}$ Patients with post-stroke depression (PSD) have worse functional recovery, ${ }^{29}$ a higher risk for cognitive impairment, ${ }^{7}$ and higher mortality risk. ${ }^{30,31}$ 
Early studies implied that PSD is mainly associated with anterior lesions in the left hemisphere, however, a major systematic review and meta-analysis found no support for this 'lesions location hypothesis..32 Vataja et al. ${ }^{33}$ suggested that lesions in fronto-subcortical regions in general are involved in developing PSD, often accompanied by impairments in executive functioning, leading to what has been called the 'depression-executive dysfunction syndrome'. Other factors that have been associated with the development of PSD are cognitive impairment, stroke severity, physicial disability, and pre-stroke depression and anxiety. ${ }^{7}$ Diminished QoL and also low social support can both contribute independently to the severity of depression. ${ }^{34} \mathrm{~A}$ study by van Mierlo et al. ${ }^{35}$ investigated the association between a broad range of psychological factors and PSD with a multivariable logistic regression analysis. They showed that more passive coping and more helplessness, less acceptance and less perceived benefits were all significantly and independently associated with symptoms of post-stroke depression. Furthermore, fatigue after stroke has been associated with PSD, although not all patients with fatigue develop symptoms of depression and vice versa. ${ }^{28}$

Neuroinflammation might also play a role in the underlying mechanisms of PSD. Levels of CRP, soluble E selectin, CD40 ligand, interleukin-6, tumor necrosis factor alpha, and high sensitivity CRP are deregulated in patients diagnosed with major depressive disorder. ${ }^{14,18,22,23,36-38}$ In addition, high plasma levels of neopterin are found in people with depression, and might predict the development of PSD. ${ }^{39}$ Markers of endothelial function might be important as well, since increased expression of soluble intercellular adhesion molecule and soluble vascular cell adhesion molecule-1 are associated with depression. ${ }^{40-43}$ Although several inflammation markers have been related to symptoms of depression, studies examining these associations in PSD specifically are relatively scarce. Some studies found an elevation in interleukin-6 and tumor necrosis factor alpha in PSD. ${ }^{44}$ Recently, a study by Tang et al..$^{39}$ showed an association between elevated serum levels of neopterin in the acute phase after stroke and PSD at 6-month follow-up.

High plasma levels of homocysteine are known to increase the risk for cerebrovascular disease, but have also been associated with depressive disorders. Increased levels of homocysteine result in cerebrovascular disease and a deficiency in monoamine neurotransmitters, which might lead to a depressed mood. ${ }^{45}$ Homocysteine, folate and vitamin B12 are all involved in methylation reactions that are necessary for monoamine neurotransmitter production, but also the production of phospholipids and nucleotides. A deficiency in folate and vitamin B12 has also been associated with depressive disorders. ${ }^{46}$ 


\section{POST-STROKE APATHY}

Apathy has been defined as a disorder of diminished motivation, characterized by lowered initiative, restricted engagement in social interactions and activities, diminished cognitive activities, and lack of emotional response. ${ }^{47}$ It was traditionally seen as a symptom of other syndromes (e.g. depression and dementia), but mounting evidence suggests that it might be an independent syndrome with a different etiology. ${ }^{48-50}$ Post-stroke apathy (PSA) is as frequent as PSD, with a mean prevalence rate of $34.6 \%$ at 4 months post-stroke. ${ }^{5}$ However, PSA gained relatively less attention in research in comparison with PSD, and most research focuses on the difference between the two.

While PSD is suggested to be associated with left anterior lesions, ${ }^{51,52}$ PSA has been associated with right hemispheric subcortical lesions, particularly in the basal ganglia and in the anterior cingulate circuit which is involved in motivational processes. ${ }^{53-55}$ Other factors associated with PSA are older age, lower education, and severity of VCl.4,5,51,56 Furthermore, both PSD and PSA have been associated with poor functional recovery and low QoL.5,29 Overall, lack of longitudinal studies, differences between time of measurement after stroke (acute phase/chronic phase), and lack of studies with a sufficient sample size make it difficult to interpret study results.

In conclusion, several factors play a role in the development of $\mathrm{VCl}, \mathrm{PSD}$, and PSA. However, most studies investigated the underlying mechanisms in isolation and did not take into account how several factors interact with each other. The Cognition and Affect after Stroke, a Prospective Evaluation of Risks (CASPER) study incorporates a broad range of psychosocial, blood and neuroimaging markers to be able to study their role alone and in combination with each other to predict individual differences in the onset and course of the cognitive and neuropsychiatric consequences of stroke.

\section{STUDY AIMS}

The primary aim of CASPER is to identify stroke-related, cerebrovascular, neurodegenerative, (epi)genetic, endothelial and inflammation markers that are associated with VCl, PSD, and PSA in patients with ischemic or hemorrhagic stroke. The secondary aims are to investigate how the above-mentioned markers interact with each other, and to determine if patients with apathy and depression after stroke differ in pathogenesis, course, and outcome (e.g. functional outcome, neurocognitive performance, quality of life). 
The main research questions are:

1. Are stroke-specific, additional vascular, neurodegenerative, inflammatory or genetic markers a) associated with $\mathrm{VCl}$ at 3 months after stroke and b) predictive for its course over 12 months?

2. Are stroke-specific, additional vascular, neurodegenerative, inflammatory or genetic markers a) associated with PSD and PSA at 3 months after stroke and b) predictive for their course over 12 months?

3. Do the above-mentioned markers interact on VCI, PSD, and PSA outcome?

4. Which psychosocial factors are associated with the development of $\mathrm{VCI}, \mathrm{PSD}$, and PSA?

5. Do PSA and PSD differ in their pathogenesis, cognitive profile, course, and outcome?

\section{METHODS}

\section{STUDY DESIGN}

CASPER is a prospective clinical cohort study into cognitive impairments and neuropsychiatric syndromes after stroke with a follow-up of 1 year. Baseline measurements take place in the post-acute phase after stroke (i.e. 10 to 12 weeks post-stroke) to assess presence and severity of VCl, PSD, and PSA. Serial assessments take place at 6 and 12 months after baseline. The total duration of the study will be approximately 3.5 years, from June 2013 (first in) until November 2016 (last out).

\section{PATIENTS}

In total, 250 consecutive patients with either ischemic or hemorrhagic stroke are included. Patients who are admitted to the Stroke Unit of the Maastricht University Medical Center+ (MUMC+), Maastricht, the Netherlands, the Stroke Unit of Zuyderland Medical Center in Sittard-Geleen and Heerlen, the Netherlands, or those who visit the Transient Ischemic Attack clinic of MUMC+ for a non-fatal ischemic or intracerebral hemorrhagic stroke and fulfill the in- and exclusion criteria are asked to participate. Stroke is defined as a clinical stroke syndrome (sudden neurological dysfunction lasting $>24 \mathrm{~h}$, with no apparent cause other than that of vascular origin). The stroke can be a first-ever event or a recurrent supra- or infratentorial stroke in a patient who recovered from a previous event without obvious residual symptoms. Ischemic strokes might be cortical or lacunar, and could be included with or without evidence of ischemia on clinical brain 
scan. Hemorrhagic strokes are non-traumatic deep, lobar, cerebellar, or brainstem hemorrhages as evidenced by a clinical brain scan. Eligibility criteria are chosen to make sure that the sample will represent the current clinical situation (Table 1). Participation of an informant who knows the patient well enough to answer questions about his/her functioning over the last \pm 10 years is desirable but not required.

Table 1. In- and exclusion criteria

\begin{tabular}{|c|c|}
\hline Inclusion criteria & Exclusion criteria \\
\hline - Ischemic or hemorrhagic stroke & $\begin{array}{l}\text { Subarachnoidal hemorrhage, traumatic hemorrhage, } \\
\text { primary intraventricular hemorrhage and transient } \\
\text { ischemic attack }\end{array}$ \\
\hline $\begin{array}{l}\text { MMSE score } \geq 15 \\
\text { (to ensure valid testing) }\end{array}$ & - Age younger than 40 years (to exclude atypical strokes) \\
\hline - Written informed consent & $\begin{array}{l}\text { Severe aphasia (as it interferes with performance } \\
\text { on and understanding of the instructions of the } \\
\text { neuropsychological tests) }\end{array}$ \\
\hline \multirow[t]{2}{*}{$\begin{array}{l}\text { - Sufficient knowledge of the Dutch } \\
\text { language }\end{array}$} & $\begin{array}{l}\text { Evidence for pre-stroke dementia (based on clinical } \\
\text { diagnosis or IQ-CODE) in the } 5 \text { years prior to the stroke }\end{array}$ \\
\hline & $\begin{array}{l}\text { Other existing psychiatric and neurological diagnoses } \\
\text { that are known to affect cognition (Parkinson's disease, } \\
\text { bipolar disorder, epilepsy, schizophrenia, or substance } \\
\text { abuse) }{ }^{1}\end{array}$ \\
\hline
\end{tabular}

Note: ${ }^{1}$ Lifetime history of depression is not considered as a reason for exclusion as it might be a risk factor for post-stroke depression. Abbreviations: IQ-CODE, Informant-Questionnaire on Cognitive Decline in the Elderly; MMSE, Mini Mental State Examination.

\section{ASSESSMENTS}

The baseline measurement (TO) is scheduled 3 months post-stroke to avoid confounding effects by the acute event, including acute inflammatory responses. At T0, patients undergo a venipuncture, brain magnetic resonance imaging (MRI) scan, neuropsychological assessment and neuropsychiatric interview. They fill in several questionnaires to assess the presence and severity of PSD and PSA, and to evaluate other factors as functional ability, fatigue, personality, and QoL. A semi-structured interview with an informant is performed to provide additional information about functional outcome of the patient and presence of neuropsychiatric syndromes. Six (T1) and 12 months (T2) after T0, the neuropsychological assessment and questionnaires are repeated. The venipuncture, MRI scan, neuropsychological assessment, and neuropsychiatric interview all take place in the academic hospital in Maastricht and are performed by specially trained nurses (venipuncture), radiology assistants (MRI scan) and trained research (neuro) 
psychologists (neuropsychological assessment and neuropsychiatric interview). If a patient is not able to visit the hospital due to reduced mobility, the patients are visited at their current place of residence.

Patients who suffer from a recurrent stroke during the 12-month follow-up period will be followed-up normally, if the health status of the patient allows this, and if the patient does not suffer from severe aphasia after the recurrent stroke. Information about the type, location, and severity of the lesion of the recurrent stroke is collected, which also allows us to study the potential impact of recurrent strokes on our outcome measures. Recurrent strokes are taken into account in our analyses with longitudinal data to avoid confounding effects.

\section{CLINICAL DATA}

In a standardized case record form, data on demographics (e.g. age, gender, educational level, ethnicity, marital status), medical history (e.g. personal and family history of cardiovascular, neurological or psychiatric disorders), medication use, physical examination (e.g. weight, height, blood pressure, cholesterol), and lifestyle (smoking behavior, alcohol consumption, drug use, physical activity) are collected.

\section{NEUROPSYCHOLOGICAL ASSESSMENT AND COGNITIVE ENDPOINTS}

The neuropsychological assessment consists of a standardized battery of cognitive tests measuring specific cognitive domains: global cognition, episodic memory, working memory, information processing speed, executive functioning, visuoconstruction, neglect, premorbid IQ, and language (dysphasia). An overview of all test instruments and their cognitive domains is presented in Table 2. The neuropsychological tests are administered according to a standardized test protocol by trained research (neuro)psychologists.

$\mathrm{VCl}$ is defined as a score $\leq 1.5$ standard deviations below the general population mean in one or more cognitive domains, based on available norm scores for age, gender, and level of education for the Dutch general population. In addition, there should not be any interference in daily activities and no diagnosis of dementia according to DSM-5 criteria. ${ }^{57}$ The impairments have to represent a significant decline from premorbid levels of functioning. The diagnosis and type of dementia is made by an experienced neuropsychiatrist or neuropsychologist based on DSM-5 criteria. ${ }^{57} \mathrm{~A}$ consensus meeting is arranged when there is a discrepancy in diagnosis, and if no consensus can be reached, the patient will be considered not demented. The diagnosis of $A D$ is made according to the standardized clinical criteria for AD based on the National Institute for Neurological and Communicative 
Disorders and Stroke-Alzheimers Disease and Related Disorders Association (NINCDS-ADRDA) criteria. ${ }^{58}$ The diagnosis of $\mathrm{VaD}$ is made according to the criteria proposed by the National Institute of Neurological Disorders and Strokes Association Internationale pour la Recherche et l'Enseignement en Neurosciences (NINDS-AIREN). ${ }^{59}$

The Informant Questionnaire on Cognitive Decline in the Elderly (IO-CODE; short form) is used to detect possible dementia prior to the index stroke, and is rated retrospectively (past 5 years prior to the index stroke), ${ }^{60}$ in combination with information from medical records. A cut-off score $\geq 3.60$ is used to indicate possible dementia prior to the stroke, as has been used in a previous study with stroke patients. ${ }^{61}$

\section{NEUROPSYCHIATRIC ASSESSMENT}

\section{Depression}

The symptoms of a major depressive disorder according to DSM-IV and DSM-5 are assessed with the Mini International Neuropsychiatric Interview (M.I.N.I.). ${ }^{71}$ The MINI is a semi-structured interview administered to the patient and consists of three different parts, which aim to determine whether the patient can be diagnosed with a current major depressive disorder, a depressive episode lifetime, and a current dysthymic disorder. The clinician-rated Montgomery-Åsberg Depression Rating Scale (MADRS) is used to assess the severity of symptoms of depression. ${ }^{72}$ This rating scale consists of 10 items to evaluate the severity of depressive symptoms and is filled out by the clinician. ${ }^{72}$ In addition, the Hospital Anxiety and Depression Scale (HADS), a 14-item self-report scale, is used to identify clinically significant levels of anxiety and depression. ${ }^{73}$ According to a study by Kang et al., ${ }^{81}$ the HADS and MADRS are especially valid as screening instrument for PSD (in both the acute and chronic phase), since these scales focus less on the presence of somatic symptoms, resulting in less misclassification.

\section{Apathy}

The Apathy Evaluation Scale (AES) is an 18-item scale used to evaluate the presence and severity of apathy. ${ }^{75}$ Three different versions of the AES are available, a clinician-rated (administered to the patient), informant-rated, and a patient-rated version. The AES has been rated as the most favorable instrument for assessing apathy, with the clinician-rated version being most valid of the three different versions. ${ }^{82}$ Both the informant-rated and clinician-rated version (administered to the patient) of the AES are used in the CASPER study. 


\section{Generic}

The Neuropsychiatric Inventory (NPI) is administered as a semi-structured interview to the informant of the patient. ${ }^{74}$ The NPI is originally developed to evaluate neuropsychiatric symptoms in dementia, but is also frequently used in the stroke population. ${ }^{28}$ It evaluates the presence and severity of 12 different neuropsychiatric symptoms (delusions, hallucinations, agitation, depression, anxiety, euphoria, apathy, disinhibition, irritability, aberrant motor behavior, sleep, and appetite). ${ }^{83}$ The validity and reliability of the NPI, including the Dutch version, has been well established. ${ }^{84,85}$

Table 2. Neuropsychological measures and neuropsychiatric questionnaires

\begin{tabular}{|c|c|c|c|c|}
\hline & Instrument & $\mathrm{TO}^{\mathrm{a}}$ & $\mathrm{T} 1^{\mathrm{a}}$ & $\mathrm{T}^{\mathrm{a}}$ \\
\hline Global cognition & MMSE$^{62}$ & $x$ & $x$ & $X$ \\
\hline Episodic Memory & 15-Word Verbal Learning Test ${ }^{63}$ & $x$ & $x$ & $x$ \\
\hline Working memory & Digit-span of the WAIS-III64 & $x$ & $x$ & $x$ \\
\hline $\begin{array}{l}\text { Information processing } \\
\text { speed }\end{array}$ & $\begin{array}{l}\text { Digit Symbol Substitution Test (DSST) } \\
\text { Trail Making Test (TMT; part A and B) })^{64,65}\end{array}$ & $X$ & $x$ & $x$ \\
\hline Executive functioning & $\begin{array}{l}\text { TMT (interference), BADS Zoo Map \& Key Search, } \\
\text { Verbal Fluency ( } 60 \text { seconds; animals and } \\
\text { professions) } 65-67\end{array}$ & $x$ & $X$ & $X$ \\
\hline Visuo-construction & Clock drawing ${ }^{67}$ & $x$ & $X$ & $x$ \\
\hline Neglect & Star cancellation 68 & $x$ & & \\
\hline Premorbid IQ & $\mathrm{NLV}^{69}$ & $x$ & & \\
\hline Language / aphasia & Boston Naming Test ${ }^{70}$ & $x$ & & \\
\hline Depression & MINI, MADRS, HADS, NPI $71-74$ & $x$ & $x$ & $x$ \\
\hline Apathy & AES, NPI 74,75 & $x$ & $X$ & $x$ \\
\hline Premorbid dementia & $10 \mathrm{CODE}^{60}$ & $x$ & & \\
\hline Functional ability & Barthel Index, Lawton ${ }^{76,77}$ & $x$ & $X$ & $X$ \\
\hline Quality of life & SS-QoL ${ }^{78}$ & $x$ & $x$ & $x$ \\
\hline Fatigue & $\mathrm{FSS}^{79}$ & $x$ & $x$ & $x$ \\
\hline Personality & $\mathrm{NEO}-\mathrm{FFI}^{80}$ & $x$ & & \\
\hline
\end{tabular}

aT0: baseline measurement, T1: 6-month follow-up, T2: 12-month follow-up.

Abbreviations: AES, Apathy Evaluation Scale; BADS, Behavioral Assessment of the Dysexecutive Syndrome; FSS, Fatigue Severity Scale; HADS, Hospital Anxiety and Depression Scale; IQ-CODE, Informant-Questionnaire on Cognitive Decline in the Elderly; MADRS, Montgomery-Åsberg Depression Rating Scale; MINI, Mini International Neuropsychiatric Interview; MMSE, Mini-Mental State Examination; NEO-FFI, NEO Five Factory Inventory; NLV, Nederlandse leestest voor volwassenen (Dutch version of the National Adult Reading Test); NPI, Neuropsychiatric Inventory; SS-Qol, Stroke Specific Quality of Life; WAIS; Wechsler Adult Intelligence Scale. 


\section{ADDITIONAL INTERVIEWS}

Fatigue after stroke is assessed with the Fatigue Severity Scale (FSS). ${ }^{79}$ This 9-item self-rating scale can be answered on a 7-point scale, with higher scores representing more fatigue. The Barthel Index ${ }^{77}$ is used to evaluate impairments in activities of daily living (ADL), and the Lawton Scale ${ }^{76}$ is used to assess impairment in instrumental ADL. Furthermore, the Stroke Specific Quality of Life Scale (SSQoL) is used to evaluate health-related QoL, which is defined as the physical, psychological, and social aspects of life that can be influenced by a change in health status. ${ }^{78}$ The NEO Five Factor Inventory (NEO-FFI) is used to assess the bigfive dimensions of personality, which are extraversion, neuroticism, openness to new experiences, agreeableness, and conscientiousness. ${ }^{80}$

\section{NEUROIMAGING}

A standardized brain MRI protocol is used as described in Table 3. The MRI scans are all performed on the same 3.0 Tesla head-only scanner equipped with an 8-channel head coil (Philips Achieva, Philips Medical Systems, Best, the Netherlands), using the same standardized scanning protocol for every patient. This protocol has been optimized for stroke and WMH segmentation, as well as segmentation of the hippocampus. ${ }^{86}$ One scanning session takes approximately 30 min. After acquisition of the MR images, an anonymisation procedure is performed, to make sure that all imaging data are pseudonymised.

Table 3. Brain MRI protocol

\begin{tabular}{|c|c|c|c|c|c|c|c|}
\hline $\begin{array}{l}\text { Sequence name / } \\
\text { Acquisition method }\end{array}$ & $\begin{array}{l}\text { Field } \\
\text { of View } \\
(\mathrm{mm})\end{array}$ & Matrix & Slices & $\begin{array}{l}\text { Inter-slice } \\
\text { Gap (mm) }\end{array}$ & $\begin{array}{l}\text { Thickness } \\
(\mathrm{mm})\end{array}$ & $\begin{array}{l}\text { Voxel } \\
(\mathrm{mm})\end{array}$ & $\mathrm{TR} / \mathrm{TE} / \mathrm{TI}$ (ms) \\
\hline $\begin{array}{l}\text { Coronal 3D } \\
\text { T1-weighted (TFE) }\end{array}$ & $240 \times 240$ & $240 \times 240$ & 160 & 0 & 1 & $1 \times 1 \times 1$ & $8.2 / 3.8$ \\
\hline $\begin{array}{l}\text { 2D Axial } \\
\text { T2-weigthed (FSE) }\end{array}$ & $230 \times 185$ & $328 \times 225$ & 48 & 0 & 3 & $0.7 \times 0.8 \times 3$ & $3000 / 80$ \\
\hline $\begin{array}{l}\text { 3D fluid-attenuation } \\
\text { inversion recovery }\end{array}$ & $250 \times 250$ & $228 \times 226$ & 283 & 0 & 1.1 & $0.6 \times 1.1 \times 1.1$ & $8000 / 332 / 2400$ \\
\hline $\begin{array}{l}\text { T2* diffusion } \\
\text { weighted imaging } \\
\text { gradient echo EPI }\end{array}$ & $240 \times 240$ & $128 \times 128$ & 28 & 0 & 5 & $1.85 \times 1.85 \times 5$ & $10000 / 72 / 2400$ \\
\hline SWI & $220 \times 180$ & $220 \times 180$ & 260 & 0 & 0.5 & $1 \times 1 \times 0.5$ & $16 / 22$ \\
\hline
\end{tabular}

Abbreviations: TR, repetition time; TE, echo time; TI, inversion time; TFE, turbo field echo; FSE, fast spin echo; EPI, echo planar imaging; SWI, susceptibility weighted imaging. 


\section{MRI MARKERS}

Several stroke characteristics are visually scored by an experienced vascular neurologist and consist of: presence and type of symptomatic stroke lesion (ischemic/hemorrhage), location of the lesion, number, type, and location of old infarcts and old hemorrhages, number and location of microbleeds, and degree of perivascular spaces in basal ganglia and centrum semiovale. The Fazekas visual rating scale is used to score deep and periventricular $\mathrm{WMH} .{ }^{87}$ In addition, a semiautomatic brain tissue segmentation program, based on the program used in the Rotterdam scan study, ${ }^{88}$ is used to measure $\mathrm{WMH}$ volumes and to calculate the volume of global brain atrophy, corrected for total intracranial volume. The accuracy of this semi-automated method appeared to be within the range of the inter-observer variability of fully manual segmentations but is less timeconsuming while still requiring some manual correcting. The medial temporal lobe atrophy (MTA) visual rating scale ${ }^{89}$ is used to indicate the level of atrophy in the medial temporal lobes (ranging from 0 to 4), separately for the left and the right hemisphere. Furthermore, hippocampal volume is measured based on atlas registration with high accuracy using the robust and validated method of learning embeddings for atlas propagation (LEAP)..$^{90,91}$

\section{BLOOD MARKERS}

At baseline, fasting blood samples are taken. All patients take their morning medication after the venipuncture. In total, $35.5 \mathrm{~mL}$ of blood are taken and directly analyzed or stored. We measure several inflammation markers. In addition, standard lab procedures to assess levels of glucose, cholesterol (total, high-density lipoprotein cholesterol, low-density lipoprotein cholesterol), and triglycerides will be performed directly after the venipuncture. ${ }^{92-94}$ Biomaterial (including deoxyribonucleic acid and ribonucleic acid) is stored anonymously for 15 years after the end of the study. Table 4 lists the blood markers investigated in CASPER. 
Table 4. Blood sampling procedure

\begin{tabular}{|c|c|c|}
\hline Tubes & Blood markers & Procedure \\
\hline $\begin{array}{l}\text { EDTA tube } \\
(4 \times 6.0 \mathrm{~mL})\end{array}$ & $\begin{array}{l}\text { Myeloperoxidase, neopterin, soluble } \\
\text { intercellular adhesion molecule, } \\
\text { soluble vascular cell adhesion } \\
\text { molecule-1, soluble E selectin, soluble } \\
\text { P selectin, CD40 ligand, interleukin-6, } \\
\text { tumor necrosis factor alpha, } \\
\text { Homocysteine }\end{array}$ & $\begin{array}{l}\text { Transported on ice to the Biobank } \\
\text { Maastricht UMC+ where the } \\
\text { blood is preprocessed before } \\
\text { storing. EDTA plasma is } \\
\text { centrifuged and stored at }-80^{\circ} \mathrm{C} \\
\text { until analysis. }\end{array}$ \\
\hline $\begin{array}{l}\text { Natriumfluoride/EDTA } \\
\text { tube }(1 \times 4.0 \mathrm{~mL}) \\
\text { Coagulation tube } \\
(1 \times 5.0 \mathrm{~mL})\end{array}$ & $\begin{array}{l}\text { Glucose, total cholesterol } \\
\text { high-density lipoprotein-cholesterol, } \\
\text { low-density lipoprotein-cholesterol, } \\
\text { triglyceride, folate, vitamine B12, } \\
\text { high sensitivity C-reactive protein }\end{array}$ & $\begin{array}{l}\text { Direct analyses of these markers } \\
\text { are performed at the laboratory } \\
\text { on the day of sample collection } \\
\text { after centrifuging. }\end{array}$ \\
\hline $\begin{array}{l}\text { Buffycoats from the } \\
4 \text { EDTA tubes for } \\
\text { DNA extraction }\end{array}$ & $\begin{array}{l}\text { Apoliprotein E, clusterin, } \\
\text { phosphatidylinositol binding } \\
\text { clathrin assembly protein, encoding } \\
\text { complement [3b/4b] receptor } 1, \\
\text { angiotensin-converting enzyme, } \\
\text { methylenetetrahydrofolate reductase }\end{array}$ & $\begin{array}{l}\text { Buffycoats are taken from four } \\
\text { EDTA tubes and separately stored } \\
\text { at the Biobank until analysis. }\end{array}$ \\
\hline $\begin{array}{l}\text { Epigenetics } 1 \\
\text { PAXgene tube } \\
(2.5 \mathrm{~mL})\end{array}$ & RNA & $\begin{array}{l}\text { Blood is collected in PAXgene } \\
\text { tubes to ensure long-term } \\
\text { stability of ribonucleic acid and } \\
\text { the tubes are stored at the } \\
\text { Biobank. RNA extraction and } \\
\text { subsequent analysis of expression } \\
\text { of inflammatory and stroke-related } \\
\text { genes at the mRNA level will be } \\
\text { done. }\end{array}$ \\
\hline
\end{tabular}

Abbreviations: $\mathrm{mL}$, milliliter; EDTA, ethylenediaminetetraacetic acid; DNA, deoxyribonucleic acid; RNA, ribonucleic acid; $m R N A$, messenger ribonucleic acid.

\section{ETHICS, CONSENT AND PERMISSIONS}

The study protocol has been approved by the Medical Ethics Committee of MUMC+. The research is performed according to the principles of the Declaration of Helsinki 59th WMA General Assembly, Seoul, (October 2008) and in accordance with the Dutch Medical Research Involving Human Subjects Act (WMO). All eligible patients are informed about the aim of the study and both the patient and informant receive an information letter. All patients receive enough time to consider participation and if they agree to participate, an appointment is scheduled to sign informed consent. There are no risks for the patients participating in this study. All assessments in the study consist of routine operations and all patient data are pseudonymised. Contraindications for MRI are checked for every patient, and patients with contra- 
indications are excluded for MRI. If a patient decides to withdraw from the study, this has no consequences or risks for the patients. Patients who refuse follow-up assessment are contacted to determine the reason for refusal.

\section{POWER CALCULATION AND SAMPLE SIZE}

Based on results of the CODAS study, ${ }^{95}$ a previous study with stroke patients of our research group, it is expected that $50 \%$ of the stroke patients will have $\mathrm{VCl}$ and $10 \%$ will have $\mathrm{VaD}$ after one year. Furthermore, it is expected that $35 \%$ of the patients will have minor or major depression after one year post-stroke. ${ }^{96} \mathrm{~A}$ power calculation was performed with the freeware program $G^{*}$ Power version 3.1 (http://www.gpower.hhu.de) assuming categorical independent variables to which $50 \%$ are exposed and $50 \%$ are not exposed (median-split), since no reliable estimates are available for our main predictors from literature (MRI, inflammation and genetics). Based on a 1 -year follow-up, a two-sided alpha level of .05, and an expected drop-out rate of $20 \%$ based on the CODAS study (about $10 \%$ due to death), a sample size of 250 resulted in a power of $86 \%$ to detect a relative risk of 2.00 , or $80 \%$ to detect a relative risk of 1.89 for PSD, and a $96 \%$ power to detect a relative risk of 2.00 , or $80 \%$ power to detect a relative risk of 1.70 for $\mathrm{VCl}$.

\section{STATISTICAL ANALYSES}

Categorical data will be presented on group level expressed in absolute numbers and percentages, whereas continuous data will be presented by their mean, confidence interval, and standard deviation. In case of non-normality, continuous data will be presented as medians with their corresponding inter-quartile range. Differences between groups will be analyzed using $t$-tests for continuous variables or appropriate non-parametric counterparts, and chi-square tests for categorical variables. Pearson's correlation coefficient will be used to assess the association between two continuous variables. Random effects models will be used to measure cognitive decline from baseline to follow-up, which has the advantage over repeated measures ANOVA that it is not restricted to study completers. The effect of baseline predictors on PSD, PSA, and VCl will be estimated with Poisson and Cox regression, taking into account differences in individual follow-up time. Furthermore, multivariate finite mixture modeling will be used to test the existence of separate PSD and PSA subtypes, using lesion location, WMH, inflammation markers, genetic risk factors, functional impairment, and cognitive functioning as subtype indicators. Age, gender, and education will be included as covariates in the multivariate models. Based on theory and statistical evaluation to see whether a variable might be a probable confounder it will be decided which additional covariates will be added on each analysis. 


\section{DISCUSSION}

The CASPER study is a prospective clinical cohort study examining predictors of onset and course of $\mathrm{VCl}, \mathrm{PSD}$, and PSA using a deep phenotyping approach. It is an observational study with minimal risks for the participating patients. The longitudinal design creates the possibility to study independent predictors of VCl, PSD, and PSA. The CASPER study provides the possibility to study the course of these conditions and potential bidirectional relationships between them. A relatively large sample size and an MRI protocol that uses state-of-the-art volumetry of WMH and hippocampal atrophy make it more likely to find subtle differences in the brain's macrostructure. Therefore, the CASPER study has an additional value to earlier stroke cohort studies (e.g. Sydney Stroke Study, The FogartyMexico Stroke Cohort, CODAS). 10,95,97 Furthermore, CASPER includes extensive blood-based biomarker data which results in a rich phenotyping approach generating both blood-based biomarker data and clinical brain MRI data, creating the possibility to study cerebrovascular, neurodegenerative, inflammatory, and genetic markers, and their inter-relationships. This is valuable since this gives a better account of how these changes occur in patients, namely in combination. Hence, the combination of these markers may predict individual differences in the development of $\mathrm{VCl}, \mathrm{PSD}$, and PSA better than each marker in isolation. Taken together, these methodological strengths promise to yield novel insights into the underlying mechanisms of the burdensome consequences of stroke.

However, some limitations of the study protocol have to be acknowledged here. Although the extensive in- and exclusion criteria were formulated with caution, they can possibly result in a less representative group of patients compared to the general stroke population. For instance, patients who are aphasic or who have preexistent cognitive disturbances are excluded. No data are collected in the acute phase after stroke, which could yield additional information on the association between $\mathrm{VCl}, \mathrm{PSD}, \mathrm{PSA}$ and the earlier mentioned investigated markers, but a post-acute baseline time point is chosen to avoid confounding effects in the acute phase (e.g. generic acute inflammatory response, sickness behavior). MRI scans and venipuncture are only performed on T0, since it is not feasible due to logistical and financial reasons to repeat them at T1 and T2. However, if these data could also have been collected more information on the causes of VCl, PSD, and PSA could have been gathered, e.g. by linking longitudinal changes on MRI to changes in outcomes. Furthermore, patients with severe stroke are less probable to participate in the study, as the assessment is quite extensive. This can result in a cohort of patients with relatively mild stroke, which may influence the frequency of $\mathrm{VCl}, \mathrm{PSD}$, and PSA. Both ischemic and hemorrhagic strokes are included to 
increase the inclusion rate, however, due to the different etiologies of these strokes, it is likely that this limits the generalizability of the results. Therefore, we have to correct for stroke type in our analyses to take this into account. It is also likely that patients with depression or apathy after stroke are less motivated to participate in the study, resulting in an underestimation of the PSD and PSA prevalence in the study sample. In addition, the current follow-up period of 1 year allows testing predictions in the post-acute to early chronic phase, but is too short to study longterm consequences. Finally, CASPER allows for in-depth phenotyping and also covers a broad spectrum of factors impacting on the consequences of stroke, but it is not powered to test for more subtle associations or to use complex genetic models or testing of rare variants. Therefore, the study is also designed as to allow harmonization with other stroke cohorts in larger meta-studies as part of the STROKOG consortium (https://cheba.unsw.edu.au/group/strokog).

In conclusion, the CASPER study allows to study the role of several markers, consisting of cerebrovascular, neurodegenerative, and inflammatory markers, and their inter-relationships, in the development of VCl, PSD, and PSA. Furthermore, the longitudinal design and sample size provide the possibility to study the course of PSA, PSD, and $\mathrm{VCl}$ and explore individual differences in their development. Better understanding of the role of all of these markers will make it possible to provide new treatment techniques or adapt existing treatment techniques, both on a biological and psychological level, which can result in better $\mathrm{QoL}$ and functional outcome of stroke patients. 


\section{REFERENCES}

1. Feigin VL, Forouzanfar MH, Krishnamurthi R, Mensah GA, Connor M, Bennett DA, et al. Global and regional burden of stroke during 1990-2010: Findings from the global burden of disease study 2010. Lancet. 2014;383:245-255.

2. Lozano R, Naghavi M, Foreman K, Lim S, Shibuya K, Aboyans V, et al. Global and regional mortality from 235 causes of death for 20 age groups in 1990 and 2010: A systematic analysis for the global burden of disease study 2010. Lancet. 2013;380:2095-2128.

3. Struijs JN, van Genugten ML, Evers SM, Ament AJ, Baan CA, van den Bos GA. Modeling the future burden of stroke in the netherlands impact of aging, smoking, and hypertension. Stroke. 2005;36:1648-1655.

4. Jorge RE, Starkstein SE, Robinson RG. Apathy following stroke. Can J Psychiatry. 2010;55:350354.

5. van Dalen JW, van Charante EPM, Nederkoorn PJ, van Gool WA, Richard E. Poststroke apathy. Stroke. 2013;44:851-860.

6. Carod-Artal J, Egido JA, González JL, De Seijas EV. Quality of life among stroke survivors evaluated 1 year after stroke. Stroke. 2000;31:2995-3000.

7. Ayerbe L, Ayis S, Wolfe CD, Rudd AG. Natural history, predictors and outcomes of depression after stroke: Systematic review and meta-analysis. Br J Psychiatry. 2013;202:14-21.

8. T O'Brien J, Erkinjuntti T, Reisberg B, Roman G, Sawada T, Pantoni L, et al. Vascular cognitive impairment. Lancet Neurol. 2003;2:89-98.

9. Pendlebury ST, Rothwell PM. Prevalence, incidence, and factors associated with pre-stroke and post-stroke dementia: A systematic review and meta-analysis. Lancet Neurol. 2009;8:1006-1018.

10. Sachdev P, Brodaty H, Valenzuela M, Lorentz L, Looi J, Berman K, et al. Clinical determinants of dementia and mild cognitive impairment following ischaemic stroke: The sydney stroke study. Dem Geriatr Cogn Disord. 2006;21:275-283.

11. Pohjasvaara T, Mäntylä R, Salonen O, Aronen HJ, Ylikoski R, Hietanen M, et al. How complex interactions of ischemic brain infarcts, white matter lesions, and atrophy relate to poststroke dementia. Arch Neurol. 2000;57:1295-1300.

12. Kokmen E, Whisnant J, O'Fallon W, Chu C-P, Beard C. Dementia after ischemic stroke: A population-based study in rochester, minnesota (1960-1984). Neurology. 1996;46:154-159.

13. Richards M, Brayne C. What do we mean by alzheimer's disease? BMJ. 2010;341:865-867.

14. Spalletta G, Bossu P, Ciaramella A, Bria P, Caltagirone C, Robinson R. The etiology of poststroke depression: A review of the literature and a new hypothesis involving inflammatory cytokines. Mol Psychiatry. 2006;11:984-991.

15. Rothenburg LS, Herrmann N, Swardfager W, Black SE, Tennen G, Kiss A, et al. The relationship between inflammatory markers and post stroke cognitive impairment. J Geriatr Psychiatry Neurol. 2010;23:199-205.

16. Di Napoli M, Schwaninger M, Cappelli R, Ceccarelli E, Di Gianfilippo G, Donati C, et al. Evaluation of c-reactive protein measurement for assessing the risk and prognosis in ischemic stroke: A statement for health care professionals from the crp pooling project members. Stroke. 2005;36:1316-1329.

17. Tzikas S, Schlak D, Sopova K, Gatsiou A, Stakos D, Stamatelopoulos K, et al. Increased myeloperoxidase plasma levels in patients with alzheimer's disease. J Alzheimers Dis. 2014;39:557-564.

18. Rajagopalan S, Brook R, Rubenfire M, Pitt E, Young E, Pitt B. Abnormal brachial artery flowmediated vasodilation in young adults with major depression. Am J Cardiology. 2001;88:196198.

19. Corsi MM, Licastro F, Porcellini E, Dogliotti G, Galliera E, Lamont JL, et al. Reduced plasma levels of $p$-selectin and I-selectin in a pilot study from alzheimer disease: Relationship with neurodegeneration. Biogerontology. 2011;12:451-454. 
20. Togo $T$, Akiyama H, Kondo H, Ikeda K, Kato M, Iseki E, et al. Expression of cd40 in the brain of alzheimer's disease and other neurological diseases. Brain Res. 2000;885:117-121.

21. Engelhart MJ, Geerlings MI, Meijer J, Kiliaan A, Ruitenberg A, van Swieten JC, et al. Inflammatory proteins in plasma and the risk of dementia: The rotterdam study. Arch Neurology. 2004;61:668672.

22. Tarkowski E, Liljeroth A-M, Minthon L, Tarkowski A, Wallin A, Blennow K. Cerebral pattern of proand anti-inflammatory cytokines in dementias. Brain Res Bull. 2003;61:255-260.

23. Komulainen P, Lakka TA, Kivipelto M, Hassinen M, Penttilä IM, Helkala E-L, et al. Serum high sensitivity c-reactive protein and cognitive function in elderly women. Age Ageing. 2007;36:443448.

24. Schrijvers EM, Schürmann B, Koudstaal PJ, van den Bussche H, Van Duijn CM, Hentschel F, et al. Genome-wide association study of vascular dementia. Stroke. 2012;43:315-319.

25. Chapman J, Wang N, Treves TA, Korczyn AD, Bornstein NM. Ace, mthfr, factor v leiden, and apoe polymorphisms in patients with vascular and alzheimer's dementia. Stroke. 1998;29:1401-1404.

26. Bertram L, Lill CM, Tanzi RE. The genetics of alzheimer disease: Back to the future. Neuron. 2010;68:270-281.

27. Hackett ML, Yapa C, Parag V, Anderson CS. Frequency of depression after stroke: A systematic review of observational studies. Stroke. 2005;36:1330-1340.

28. Hackett ML, Köhler S, T O'Brien J, Mead GE. Neuropsychiatric outcomes of stroke. Lancet Neurol. 2014;13:525-534.

29. Hama S, Yamashita H, Shigenobu M, Watanabe A, Hiramoto K, Kurisu K, et al. Depression or apathy and functional recovery after stroke. Int J Geriatr Psychiatry. 2007;22:1046-1051.

30. Köhler S, Verhey F, Weyerer S, Wiese B, Heser K, Wagner M, et al. Depression, non-fatal stroke and all-cause mortality in old age: A prospective cohort study of primary care patients. J Affect Disord. 2013;150:63-69.

31. Bartoli F, Lillia N, Lax A, Crocamo C, Mantero V, Carrà G, et al. Depression after stroke and risk of mortality: A systematic review and meta-analysis. Stroke Res Treat. 2013;2013:862978-862988.

32. Carson AJ, MacHale S, Allen K, Lawrie SM, Dennis M, House A, et al. Depression after stroke and lesion location: A systematic review. Lancet. 2000;356:122-126.

33. Vataja R, Pohjasvaara T, Mäntylä R, Ylikoski R, Leskelä M, Kalska H, et al. Depression-executive dysfunction syndrome in stroke patients. Am J Geriatr Psychiatry. 2005;13:99-107.

34. Taylor-Piliae RE, Hepworth JT, Coull BM. Predictors of depressive symptoms among communitydwelling stroke survivors. J Cardiovasc Nurs. 2013;28:460-467.

35. van Mierlo ML, van Heugten CM, Post MW, de Kort PL, Visser-Meily JM. Psychological factors determine depressive symptomatology after stroke. Arch Phys Med Rehabil. 2015;96:1064-1070.

36. Neubauer H, Petrak F, Zahn D, Pepinghege F, Hägele A-K, Pirkl P-A, et al. Newly diagnosed depression is associated with increased beta-thromboglobulin levels and increased expression of platelet activation markers and platelet derived cd40-cd40l. J Psychiatr Res. 2013;47:865-871.

37. Howren MB, Lamkin DM, Suls J. Associations of depression with c-reactive protein, il-1, and il-6: A meta-analysis. Psychosom Med. 2009;71:171-186.

38. Tuglu C, Kara SH, Caliyurt O, Vardar E, Abay E. Increased serum tumor necrosis factor-alpha levels and treatment response in major depressive disorder. Psychopharmacology. 2003;170:429-433.

39. Tang C-Z, Zhang Y-L, Wang W-S, Li W-G, Shi J-P. Elevated serum levels of neopterin at admission predicts depression after acute ischemic stroke: A 6-month follow-up study. Mol Neurobiology. 2015:1-11.

40. Lespérance $F$, Frasure-Smith $N$, Théroux $P$, Irwin $M$. The association between major depression and levels of soluble intercellular adhesion molecule 1, interleukin-6, and c-reactive protein in patients with recent acute coronary syndromes. Am J Psychiatry. 2004;161:271-277.

41. Schaefer $M$, Horn $M$, Schmidt F, Schmid-Wendtner MH, Volkenandt $M$, Ackenheil $M$, et al. Correlation between sicam-1 and depressive. symptoms during adjuvant treatment of melanoma with interferon-a. Brain, Behavior Immunity. 2004;18:555-562. 
42. Motivala SJ, Sarfatti A, Olmos L, Irwin MR. Inflammatory markers and sleep disturbance in major depression. Psychosom Med. 2005;67:187-194.

43. Dimopoulos N, Piperi C, Salonicioti A, Mitsonis C, Liappas I, Lea RW, et al. Elevation of plasma concentration of adhesion molecules in late-life depression. Int J Geriatr Psychiatry. 2006;21:965971.

44. Zhan Y, Yang Y-T, You H-M, Cao D, Liu C-Y, Zhou C-J, et al. Plasma-based proteomics reveals lipid metabolic and immunoregulatory dysregulation in post-stroke depression. Eur Psychiatry. 2014;29:307-315.

45. Folstein M, Liu T, Peter I, Buel J, Arsenault L, Scott T, et al. The homocysteine hypothesis of depression. Am J Psychiatry. 2007;164:861-867.

46. Bottiglieri T, Laundy M, Crellin R, Toone BK, Carney MW, Reynolds EH. Homocysteine, folate, methylation, and monoamine metabolism in depression. J Neurol, Neurosurg Psychiatry. 2000;69:228-232.

47. Marin RS. Differential diagnosis and classification of apathy. Am J Psychiatry. 1990;147:22-30.

48. Roth RM, Flashman LA, McAllister TW. Apathy and its treatment. Curr Treat Options Neurol. 2007;9:363-370.

49. Withall A, Brodaty H, Altendorf A, Sachdev PS. A longitudinal study examining the independence of apathy and depression after stroke: The sydney stroke study. Int Psychogeriatr. 2011;23:264273.

50. Marin RS. Apathy: A neuropsychiatric syndrome. J Neuropsychiatr Clin Neurosci. 1991;3:243254.

51. Brodaty H, Sachdev P, Withall A, Altendorf A, Valenzuela M, Lorentz L. Frequency and clinical, neuropsychological and neuroimaging correlates of apathy following stroke: The Sydney stroke study. Psychol Med. 2005;35:1707-1716.

52. Hama S, Yamashita H, Shigenobu M, Watanabe A, Kurisu K, Yamawaki S, et al. Post-stroke affective or apathetic depression and lesion location: Left frontal lobe and bilateral basal ganglia. Eur Arch Psychiatr Clin Neurosci. 2007;257:149-152.

53. Caeiro L, Ferro J, Figueira M. Apathy in acute stroke patients. Eur J Neurol. 2012;19:291-297.

54. Andersson S, Krogstad J, Finset A. Apathy and depressed mood in acquired brain damage: Relationship to lesion localization and psychophysiological reactivity. Psychol Med. 1999;29:447456.

55. Okada K, Kobayashi S, Yamagata S, Takahashi K, Yamaguchi S. Poststroke apathy and regional cerebral blood flow. Stroke. 1997;28:2437-2441.

56. Mayo NE, Fellows LK, Scott SC, Cameron J, Wood-Dauphinee S. A longitudinal view of apathy and its impact after stroke. Stroke. 2009;40:3299-3307.

57. APA. Diagnostic and statistical manual of mental disorders (5th ed.). Arlington, VA: American Psychiatric Publishing; 2013.

58. McKhann G, Drachman D, Folstein M, Katzman R, Price D, Stadlan EM. Clinical diagnosis of alzheimer's disease report of the nincds-adrda work group* under the auspices of department of health and human services task force on alzheimer's disease. Neurology. 1984;34:939-939.

59. Román GC, Tatemichi TK, Erkinjuntti T, Cummings J, Masdeu J, Garcia J, et al. Vascular dementia diagnostic criteria for research studies: Report of the ninds-airen international workshop*. Neurology. 1993;43:250-260.

60. Jorm A. A short form of the informant questionnaire on cognitive decline in the elderly (IQCODE): Development and cross-validation. Psychol Med. 1994;24:145-153.

61. Nys G, Van Zandvoort M, De Kort P, Jansen B, De Haan E, Kappelle L. Cognitive disorders in acute stroke: Prevalence and clinical determinants. Cerebrovasc Dis. 2007;23:408-416.

62. Folstein MF, Folstein SE, McHugh PR. "Mini-mental state": A practical method for grading the cognitive state of patients for the clinician. J Psychiatr Res. 1975;12:189-198.

63. Rey A. L'examen clinique en psychologie. Oxford England: Presses Universitaries De France; 1958. 
64. Wechsler D. Wechsler memory scale - 3rd edition: Administration and scoring manual. San Antonio, TX: The Psychological Corporation; 1997.

65. Reitan RM. Validity of the trail making test as an indicator of organic brain damage. Percept Mot Skills. 1958;8:271-276.

66. Wilson BA, Alderman N, Burgess PW, Emslie H, Evans JJ. Behavioural assessment of the dysexecutive syndrome (bads). J Occ Psychol Employment Disability. 2003;5:33-37.

67. Lezak MD. Neuropsychological assessment. 3rd edition. New York: Oxford university Press; 1995.

68. Halligan P, Marshall J, Wade D. Visuospatial neglect: Underlying factors and test sensitivity. Lancet. 1989;334:908-911.

69. Schmand BA, Bakker D, Saan RJ, Louman J. De nederlandse leestest voor volwassenen: Een maat voor het premorbide intelligentieniveau. Tijdschrift voor Gerontologie en Geriatrie. 1991:127138.

70. Kaplan E, Goodglass H, Weintraub S. The boston naming test, 2nd edition. Philadelphia: Lea \& Febiger; 1983.

71. Overbeek I, Schruers K, Griez E. Mini international neuropsychiatric interview: Nederlandse versie 5.0.0. DSM-IV [Dutch version]. Maastricht, The Netherlands: Universiteit Maastricht. 1999

72. Montgomery SA, Asberg M. A new depression scale designed to be sensitive to change. Brit J Psychiatry. 1979;134:382-389.

73. Zigmond AS, Snaith RP. The hospital anxiety and depression scale. Acta Psychiatr Scand. 1983;67:361-370.

74. Cummings JL. The neuropsychiatric inventory assessing psychopathology in dementia patients. Neurology. 1997;48:10-16.

75. Marin RS, Biedrzycki RC, Firinciogullari S. Reliability and validity of the apathy evaluation scale. Psychiatr Res. 1991;38:143-162.

76. Lawton MP, Brody EM. Assessment of older people: Self-maintaining and instrumental activities of daily living. Gerontologist. 1969;9:179-186.

77. Mahoney Fl. Functional evaluation: The barthel index. Md State Med J. 1965;14:61-65.

78. Post MW, Boosman H, Van Zandvoort MM, Passier PE, Rinkel GJ, Visser-Meily JM. Development and validation of a short version of the stroke specific quality of life scale. J Neurol Neurosurg Psychiatry. 2011;82:283-286.

79. Herlofson K, Larsen JP. Measuring fatigue in patients with parkinson's disease: The fatigue severity scale. Eur J of Neurol. 2002;9:595-600.

80. Costa PT, McCrae RR. The neo personality inventory: Manual, form s and form r. Odessa, FL: Psychological Assessment Resources; 1985.

81. Kang H-J, Stewart R, Kim J-M, Jang J-E, Kim S-Y, Bae K-Y, et al. Comparative validity of depression assessment scales for screening poststroke depression. J Affect Disord. 2013;147:186-191.

82. Clarke DE, Ko JY, Kuhl EA, van Reekum R, Salvador R, Marin RS. Are the available apathy measures reliable and valid? A review of the psychometric evidence. J Psychosom Res. 2011;70:73-97.

83. Aalten P, de Vugt ME, Jaspers N, Jolles J, Verhey FR. The course of neuropsychiatric symptoms in dementia. Part i: Findings from the two-year longitudinal maasbed study. Int J Geriatr Psychiatry. 2005;20:523-530.

84. Kat MG, De Jonghe JF, Aalten P, Kalisvaart C, Dröes R-M, Verhey FR. Neuropsychiatric symptoms of dementia: Psychometric aspects of the dutch version of the neuropsychiatric inventory (npi). Tijdschift voor Gerontologie en Geriatrie. 2002;33:150-155.

85. Cummings J, McPherson S. Neuropsychiatric assessment of alzheimer's disease and related dementias. Aging Clin Exp Res. 2001;13:240-246.

86. Aalten P, Ramakers IH, Biessels GJ, de Deyn PP, Koek HL, OldeRikkert MG, et al. The dutch parelsnoer institute-neurodegenerative diseases; methods, design and baseline results. BMC Neurol. 2014;14:254-261. 


\section{Chapter 3}

87. Fazekas F, Chawluk JB, Alavi A, Hurtig HI, Zimmerman RA. Mr signal abnormalities at $1.5 t$ in alzheimer's dementia and normal aging. Am J Neuroradiology. 1987;8:421-426.

88. de Boer R, Vrooman HA, van der Lijn F, Vernooij MW, Ikram MA, van der Lugt A, et al. White matter lesion extension to automatic brain tissue segmentation on mri. Neuroimage. 2009;45:11511161.

89. Scheltens $P$, Leys $D$, Barkhof $F$, Huglo D, Weinstein $H$, Vermersch $P$, et al. Atrophy of medial temporal lobes on mri in" probable" alzheimer's disease and normal ageing: Diagnostic value and neuropsychological correlates. J Neurol Neurosurg Psychiatry. 1992;55:967-972.

90. Wolz R, Aljabar P, Hajnal JV, Hammers A, Rueckert D. Leap: Learning embeddings for atlas propagation. Neuroimage. 2010;49:1316-1325.

91. Wolz R, Schwarz AJ, Yu P, Cole PE, Rueckert D, Jack CR, et al. Robustness of automated hippocampal volumetry across magnetic resonance field strengths and repeat images. Alzheimers Dementia. 2014; 10:430-438.

92. Carmena R, Duriez P, Fruchart J-C. Atherogenic lipoprotein particles in atherosclerosis. Circulation. 2004;109:|II-2-III-7.

93. Antonios N, Angiolillo DJ, Silliman S. Hypertriglyceridemia and ischemic stroke. Eur Neurol. 2008;60:269-278.

94. Beckman JA, Creager MA, Libby P. Diabetes and atherosclerosis: Epidemiology, pathophysiology, and management. JAMA. 2002;287:2570-2581.

95. Rasquin S, Verhey F, Van Oostenbrugge R, Lousberg R, Lodder J. Demographic and ct scan features related to cognitive impairment in the first year after stroke. J Neurol Neurosurg Psychiatry. 2004;75:1562-1567.

96. Bour A, Rasquin S, Aben I, Boreas A, Limburg M, Verhey F. A one-year follow-up study into the course of depression after stroke. J Nutr Health Aging. 2010;14:488-493.

97. Arauz A, Rodríguez-Agudelo Y, Sosa AL, Chávez M, Paz F, González M, et al. Vascular cognitive disorders and depression after first-ever stroke: The Fogarty-Mexico stroke cohort. Cerebrovasc Dis. 2014;38:284-289. 
The CASPER study: rationale and design 


\section{CHAPTER 4}




\section{TEMPORAL ASSOCIATIONS BETWEEN FATIGUE, DEPRESSION, AND APATHY AFTER STROKE: RESULTS OF THE COGNITION AND AFFECT AFTER STROKE, A PROSPECTIVE EVALUATION OF RISKS STUDY}

Elles Douven

Sebastian Köhler

Syenna HJ Schievink

Robert J van Oostenbrugge

Julie Staals

Frans RJ Verhey

Pauline Aalten 


\section{ABSTRACT}

Background: Post-stroke fatigue (PSF) is a form of pathological fatigue that can develop after stroke and has a negative impact on functional outcome. PSF is associated with post-stroke depression (PSD), which in turn shows similarities with post-stroke apathy (PSA). This study aimed at disentangling the temporal associations between PSF and PSD and between PSF and PSA.

Methods: A total of 250 stroke patients were included, of which 243 completed the Fatigue Severity Scale, Montgomery-Åsberg Depression Rating Scale, and Apathy Evaluation Scale at 3 months post-stroke, with follow-up measurements at 6 and 12 months after initial testing. Linear mixed models and linear regressions were performed to evaluate the temporal associations between PSF and PSD, and between PSF and PSA.

Results: PSF was present in 119 patients (49\%), of whom 62 patients also had PSD (26\%), and 21 patients (9\%) also had PSA. At baseline, PSF patients showed higher depression levels, which remained stable at follow-up. PSD patients had higher fatigue levels compared with no-PSD patients at baseline, which remained stable at follow-up. No association between apathy and fatigue was found at baseline and no interaction with time was found. Change in fatigue from baseline to 12-month follow-up was associated with change in depression and with change in apathy.

Conclusions: Bi-directional associations were found between PSF and PSD. In treatment and rehabilitation programs, early focus on presence of PSD and PSF is important, since these conditions tend to persist. As there are currently more treatment options for PSD, attention for PSD is important and might also have a beneficial effect on PSF. 


\section{INTRODUCTION}

Post-stroke fatigue (PSF) can be defined as a form of pathological fatigue, characterized by a continuous feeling of weariness, ${ }^{1}$ independent of physical exertion, ${ }^{2}$ and can develop early after stroke or in the chronic stroke phase. It is a frequent complaint after stroke, with prevalence estimates between 35 and 92\%. ${ }^{1}$ PSF has a negative impact on daily functioning and rehabilitation, ${ }^{3,4}$ resulting in higher dependency and poor quality of life. ${ }^{5}$ PSF has been associated with both biological and psychological factors, ${ }^{6}$ and is therefore hypothesized to be of multifactorial origin. ${ }^{7}$ Lack of acknowledgment of fatigue by others, including health professionals, due to its 'invisible nature', contributes to the difficulty to cope with PSF, and can cause irritability and mood changes. ${ }^{8}$

A meta-analysis of 19 cross-sectional studies showed that PSF is associated with post-stroke depression (PSD). ${ }^{9}$ Longitudinal studies found evidence that early PSD was associated with long-term PSF, ${ }^{10,11}$ and long-term PSD was also associated with long-term PSF.' Early depressive symptoms correlated positively with both early fatigue and long-term fatigue, but no long-term depression data were available. ${ }^{12}$ So, less is known about the effect of early PSF on long-term PSD, leaving the question whether a direct relationship between PSF and PSD exists, and if so, whether it is uni- or bi-directional..$^{13}$ In addition, there is large overlap in the symptoms of apathy and depression after stroke, ${ }^{14}$ and both often co-occur, ${ }^{15-17}$ suggesting they are closely related. Apathy can be defined as a condition of lack of interest and motivation, with diminished participation in activities. ${ }^{18}$ Hence, post-stroke apathy (PSA) might also be related to PSF. Therefore, the main aim of the present study is to disentangle the temporal relationships between PSF and PSD and between PSF and PSA.

\section{METHODS}

\section{STUDY POPULATION}

Participants of the present study took part in the Cognition and Affect after Stroke, a Prospective Evaluation of Risks (CASPER) study, a prospective clinical cohort study, which aims to examine predictors of post-stroke cognitive impairment, depression, and apathy, and its design has been described in detail elsewhere. ${ }^{19}$ Patients were approached to participate between June 2013 and November 2015; all patients have been admitted to the Stroke Unit of Maastricht University Medical Center (MUMC+), or Zuyderland Hospital (the Netherlands). The study protocol was approved by the Medical Ethics Committee of MUMC+. 
Patients with non-fatal first-ever ischemic stroke were included. Exclusion criteria were: hemorrhage $(n=50)$, pre-stroke dementia, pre-existing cognitive impairment, or mental retardation $(n=86)$, previous stroke $(n=54)$, age $<40$ years ( $n=17)$, neurological or psychiatric diseases other than depression that are known to affect cognition ( $n=75$ ), insufficient knowledge of the Dutch language ( $n=18)$, too severe aphasia to understand the study procedure $(n=35)$, Informant Questionnaire on Cognitive Decline in the Elderly (IQ-CODE) score ${ }^{20} \geq 3.60$ ( $n=$ 3), Mini-Mental State Examination Score (MMSE) score ${ }^{21}<15$ ( $\left.n=1\right)$, no written informed consent $(n=1)$, blindness $(n=2)$, and post-surgery stroke / post-anoxic encephalopathy $(n=2)$. After one year, initial criteria were adapted to increase response rate by additionally including patients with hemorrhage $(n=15)$ and patients with a history of stroke $\geq 3$ years, but without residual symptoms ( $n=$ 13). Patients with a history of stroke of $<3$ years or with residual symptoms from previous stroke were excluded $(n=68)$. Finally, 250 eligible patients gave their written informed consent and were enrolled in the present study (Supplementary Figure 1).

\section{PROCEDURE}

Baseline measurements were scheduled approximately 3 months post-stroke to avoid confounding effects caused by the acute stroke state. ${ }^{22}$ At baseline (TO), socio-demographic information was recorded and all patients underwent a neuropsychiatric interview in order to evaluate the presence and severity of fatigue, depression, and apathy, which was repeated at 6-month (T1) and 12-month (T2) follow-up.

\section{MEASUREMENTS}

The Fatigue Severity Scale (FSS), a 9-item self-report questionnaire, was used to assess the presence and severity of PSF. ${ }^{23}$ The FSS has been validated in stroke patients, ${ }^{24}$ and based on previous studies a cut-off score $\geq 4$ was applied to indicate PSF. 5,25

The Apathy Evaluation Scale (AES) was used to assess presence and severity of PSA, ${ }^{26,27}$ which consists of 18 items measured on a 4-point scale, with a higher total score representing a higher level of apathy. The clinician-rated AES (AES-C) version was used as outcome measure of apathy, and patients with a score $\geq 37$ on either the AES-C or informant-rated AES were classified as having clinically relevant apathy. ${ }^{28}$

The Mini International Neuropsychiatric Interview (MINI), a semi-structured interview based on DSM-IV criteria, was used to diagnose major depressive 
disorder (MDD) and minor depression (MIND). ${ }^{29}$ The Montgomery-Åsberg Depression Rating Scale (MADRS), a 10-item clinician-rated questionnaire, was used as outcome measure of depression. ${ }^{30}$ The MADRS was evaluated as a valid instrument for PSD as it focuses less on somatic symptoms and is sensitive to change. ${ }^{31}$ Patients having MDD or MIND according to the MINI, or having a MADRS score $\geq 7$ were classified as having clinically relevant depression.

The Barthel Index was used to measure impairment in activities of daily living, ${ }^{32}$ and the NEO Five Factor Inventory was used to assess the personality trait neuroticism, which was included as potential covariate in the analyses. ${ }^{33}$

\section{STATISTICAL ANALYSIS}

All statistical analyses were performed with Stata version 13.1 for Mac OS X (StataCorp, USA). An alpha level of .05 (two-sided) was used for all analyses. Baseline differences between PSF groups were tested using $X^{2}$ tests for qualitative variables and $t$-tests for quantitative variables. Pearson, point-biserial, phi, and Spearman rho correlations were used to assess correlations between variables. Variables that correlated significantly with both fatigue and depression, or fatigue and apathy were analyzed in a linear regression model. Variables that were significantly associated with both fatigue and depression, or fatigue and apathy in the linear regression models were included as potential confounders in the analyses.

Linear mixed models were performed to measure the effect of baseline PSF on depression and apathy scores at baseline, and to test whether PSF predicts change in depression and apathy scores over time from baseline to 12-month follow-up. A group (2-levels: no-PSF, PSF) by time (3-levels: T0, T1, T2) interaction was included to study differences in the course of depression between the groups. A $X^{2}$-test test of homogeneity with two degrees of freedom $(d f)$ tested the null hypothesis of no difference in rate of change over time between the groups by examining whether there is a significant group-by-time interaction. The models included a random intercept and random slope with an unstructured correlation matrix as this resulted in the best fit and the analyses were corrected for relevant confounders.

Furthermore, we tested whether change in FSS from baseline to T1 or T2 was associated with change in PSD or PSA during the same period. For this, a change score of the FSS was calculated by regressing FSS scores at follow-up on baseline FSS scores, thereby accounting for different FSS baseline levels (autoregression). The FSS change score was then used as the independent variable in a linear regression in which MADRS or AES-C scores at follow-up were regressed on baseline MADRS or AES-C scores. 
Next, the same analytic approach was adopted with FSS as outcome to measure the effect of baseline PSD or PSA on fatigue scores at baseline, and to test whether PSD or PSA predicted change in fatigue scores over time from baseline to 12-month follow-up. We additionally adjusted for the PSA-by-time or PSD-by-time interaction to study the independence of these effects. The linear mixed models only included a random intercept as this resulted in the best fit.

\section{RESULTS}

Of the 250 participants, 243 completed the FSS, MADRS, and AES-C at T0 (97.2\%), of which 210 patients (86.4\%) completed T1 and 215 patients (88.5\%) completed T2 (Supplementary Figure 1). Patients lost to follow-up at one or two time measurements $(n=27)$ did not differ from patients who completed all measurements ( $n=216$ ) (Supplementary Table 1).

Table 1 shows the baseline characteristics of the total sample ( $n=243$ ), and also separately for PSF group at baseline. PSF was present in 119 patients at T0 (49.0\%). Patients with PSF were significantly younger compared to the no-PSF group, more often reported sleep apnea, and showed higher levels of neuroticism, depression, and apathy. PSD was present in 94 patients at T0 (38.7\%), of which 13 (5.3\%) fulfilled criteria for MDD, 15 (6.2\%) for MIND, and 66 (27.2\%) had a MADRS score $\geq 7$. A significantly higher frequency of PSD and MDD was found in the PSF group in comparison with the no-PSF group, and 62 (25.5\%) of the patients experienced both PSD and PSF. PSA was present in 40 patients at T0 (16.5\%). Presence of both PSA and PSD at baseline was seen in 26 patients $(10.7 \%)$, whereas presence of both PSA and PSF at baseline was seen in 21 patients (8.6\%).

Based on correlation analyses (Supplementary Table 2) and linear regression models, only sleep apnea was associated with all outcomes, whereas neuroticism and history of depression were significantly associated with fatigue and depression scores. As sleep apnea has a direct influence on fatigue level, it was included in the primary analyses, together with baseline age, sex, and highest level of education. In the secondary analyses, also neuroticism and depression history were included to see how they influence the relationship between depression and fatigue, and apathy and fatigue. 


\section{THE COURSE OF DEPRESSION BY PSF GROUP}

In the PSF group, a significantly higher MADRS score was found at T0 $\left(X^{2}=10.68\right.$; $d f=1 ; p=.001)$, which remained stable at T1 $(p=.044)$ and T2 $(p=.008)$. There was no significant interaction between PSF and MADRS score over time $\left(\chi^{2}=1.37\right.$; $d f=2 ; p=.504 ;$ Figure $1 \mathrm{~A}$ ). Adding neuroticism and history of depression to the model did not change the results (Table 2 ).

\section{THE COURSE OF APATHY BY PSF GROUP}

No significant group effect at T0 ( $p=.121$; Table 2$)$ or interaction by time effect $\left(X^{2}=1.37 ; d f=2 ; p=.505\right)$ was found between PSF and AEC-C score (Figure 1B). While groups scored similar at T0 or T1 $(p=.218)$, a significant difference in apathy score between the PSF and no-PSF group was found at T2 $(p=.046)$. By the addition of neuroticism and history of depression to the model, the group difference in AES-C score at T2 was not significant anymore $(p=.119)$.

Table 1. Baseline characteristics

\begin{tabular}{|c|c|c|c|}
\hline Variable & Overall & No PSF & PSF \\
\hline$N(\%)$ & $243(100.0)$ & $124(51.0)$ & $119(49.0)$ \\
\hline Age (in years) & $67.41(11.76)$ & $69.44(11.33)$ & $65.30(11.87)^{\mathrm{b}}$ \\
\hline Male sex, $n(\%)$ & $157(64.6)$ & $83(66.9)$ & $74(62.2)$ \\
\hline Low education, $n(\%)$ & $100(41.3)$ & $50(40.3)$ & $50(42.4)$ \\
\hline Middle education, $n(\%)$ & $85(35.1)$ & $38(30.7)$ & $47(39.8)$ \\
\hline High education, $n(\%)$ & $57(23.6)$ & $36(29.0)$ & $21(17.8)$ \\
\hline Time since stroke (months) & $2.93(0.43)$ & $2.90(0.41)$ & $2.96(0.46)$ \\
\hline First-ever stroke, $n(\%)$ & $227(93.4)$ & $119(96.0)$ & $108(90.8)$ \\
\hline Barthel Index & $19.44(1.47)$ & $19.40(1.74)$ & $19.48(1.14)$ \\
\hline Sleep apnea, $n(\%)$ & $18(7.4)$ & $2(1.6)$ & $16(13.5)^{b}$ \\
\hline High neuroticism, $n(\%)$ & $115(48.7)$ & $69(59.0)$ & $46(38.7)^{b}$ \\
\hline Pre-stroke depression, $n(\%)$ & $55(22.6)$ & $23(18.6)$ & $32(26.9)$ \\
\hline Family history of depression, $n(\%)$ & $34(14.1)$ & $15(12.2)$ & $19(16.0)$ \\
\hline MADRS & $6.07(5.84)$ & $4.24(4.43)$ & $7.96(6.49)^{\mathrm{b}}$ \\
\hline AES-C & $26.36(7.87)$ & $25.26(6.88)$ & $27.50(8.66)^{a}$ \\
\hline FSS & $3.78(1.59)$ & $2.47(0.92)$ & $5.14(0.79)^{\mathrm{b}}$ \\
\hline MINI minor or major depression, $n(\%)$ & $28(11.5)$ & $10(8.1)$ & $18(15.1)$ \\
\hline MDD & $13(5.4)$ & $2(1.6)$ & $11(9.2)^{b}$ \\
\hline MIND & $15(6.2)$ & $8(6.5)$ & $7(5.9)$ \\
\hline PSA, $n(\%)$ & $40(16.5)$ & $19(15.3)$ & $21(17.7)$ \\
\hline PSD, $n(\%)$ & $94(38.7)$ & $32(25.8)$ & $62(52.1)^{\mathrm{b}}$ \\
\hline
\end{tabular}

Abbreviations: AES-C, Apathy Evaluation Scale Clinician-rated; FSS, Fatigue Severity Scale; MADRS, Montgomery-Åsberg Depression Rating Scale; MDD, major depressive disorder; MIND, minor depression; MINI, Mini International Neuropsychiatric Interview; PSA, post-stroke apathy; PSD, poststroke depression; PSF, post-stroke fatigue.

Note: Data are mean (SD), unless otherwise specified.

${ }^{\mathrm{a}} \mathrm{p}<0.05,{ }^{\mathrm{b}} \mathrm{p}<0.01$. 


\section{THE COURSE OF FATIGUE BY PSD GROUP}

At T0, a significantly higher FSS score was found in the PSD group $\left(X^{2}=27.25 ; d f=\right.$ $1 ; p<.001$; Table 2), which remained stable at T1 $(p<.001)$ and T2 $(p<.001)$. The overall interaction between PSD and FSS scores over time was not significant $\left(X^{2}=\right.$ 2.02; $d f=2 ; p=.365$; Figure 1C).

\section{THE COURSE OF FATIGUE BY PSA GROUP}

No significant group difference in FSS score between the PSA and no-PSA group was found at T0 $\left(X^{2}=0.20 ; d f=1 ; p=.659\right.$, Table 2$)$, T1 $(p=.571)$ or T2 ( $p=.865$; Figure 1D), and the overall interaction between PSA and FSS scores over time was not significant either $\left(X^{2}=0.17 ; d f=2 ; p=.921\right)$.
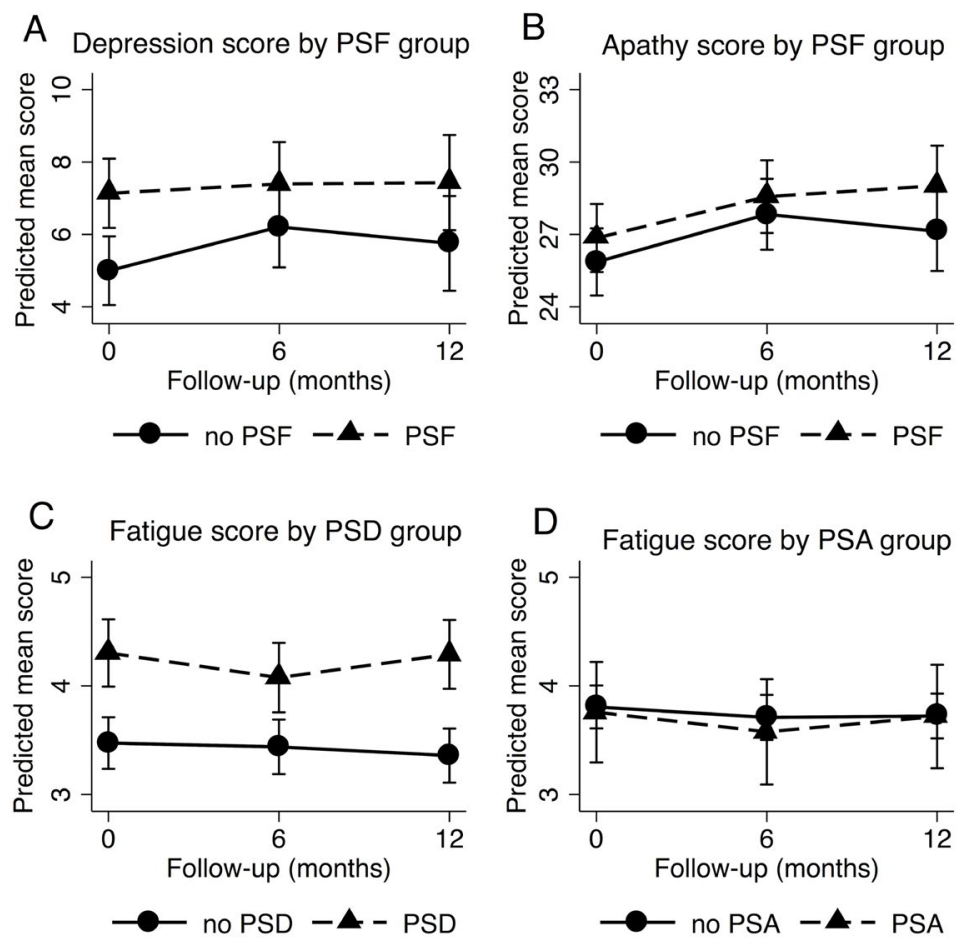

Figure 1. Course of apathy and depression by post-stroke fatigue (PSF) group and course of fatigue by post-stroke depression (PSD) and post-stroke apathy (PSA) group

(A) Course of depression scores by PSF group. (B) Course of apathy scores by PSF group. (C) Course of fatigue scores by PSD group. (D) Course of fatigue scores by PSA group. Based on random effects analyses adjusted for age at baseline, sex, highest level of education, sleep apnea, history of depression, and high neuroticism. Predicted mean scores are estimated marginal means of time by group, with all covariates fixed at their means. Higher mean scores indicate a higher level of depression, apathy, or fatigue, respectively.

Abbreviations: PSA, post-stroke apathy; PSD, post-stroke depression; PSF, post-stroke fatigue. 


\section{ASSOCIATION BETWEEN CHANGE OF FATIGUE AND CHANGE OF DEPRESSION}

Linear regression analyses showed that change in FSS score from T0 to T1 was not significantly associated with change in MADRS score from T0 to T1 ( $B=0.07, p$ $=.820$ ). However, from T0 to T2 change in FSS score was significantly associated with change in MADRS score $(B=1.47, p<.001)$. When neuroticism and history of depression were added to this model, change in FSS score remained significant $(B=1.35, p<.001)$.

\section{ASSOCIATION BETWEEN CHANGE OF FATIGUE AND CHANGE OF APATHY}

There was no significant association between change in FSS score and change in AES-C score from T0 to T1 ( $B=0.62, p=.086)$. However, from T0 to T2 change in FSS score was significantly associated with change in AES-C score $(B=1.21$, $p=.004)$. When neuroticism and history of depression were added to this model, change in FSS score remained significant $(B=0.91, p=.027)$.

Table 2. Mean differences (and 95\% confidence intervals) in depression, apathy and fatigue score and in rate of change (slopes) from baseline to follow-up

\begin{tabular}{|c|c|c|c|c|c|c|c|}
\hline \multirow[b]{3}{*}{ Parameter } & \multicolumn{7}{|c|}{ Time } \\
\hline & & \multicolumn{2}{|c|}{ Baseline } & \multicolumn{2}{|c|}{ Change T0 - T1 } & \multicolumn{2}{|c|}{ Change T0 - T2 } \\
\hline & & Difference & $95 \% \mathrm{Cl}$ & Change & $95 \% \mathrm{Cl}$ & Change & $95 \% \mathrm{Cl}$ \\
\hline \multirow{2}{*}{ MADRS by PSF } & Model $1^{c}$ & $2.75^{b}$ & $1.10,4.40$ & -0.99 & $-2.65,0.67$ & -0.44 & $-2.09,1.21$ \\
\hline & Model $2^{\mathrm{d}}$ & $2.14^{b}$ & $0.76,3.52$ & -0.95 & $-2.59,0.69$ & -0.46 & $-2.21,1.29$ \\
\hline \multirow{2}{*}{ AES-C by PSF } & Model $1^{c}$ & 1.57 & $-0.42,3.56$ & -0.22 & $-2.07,1.63$ & 0.91 & $-1.28,3.10$ \\
\hline & Model $2^{d}$ & 0.99 & $-1.04,3.03$ & -0.26 & $-2.12,1.59$ & 0.90 & $-1.25,3.05$ \\
\hline \multirow{2}{*}{ FSS by PSD } & Model $1^{c}$ & $1.08^{b}$ & $0.67,1.49$ & -0.17 & $-0.57,0.23$ & 0.13 & $-0.27,0.53$ \\
\hline & Model $2^{d}$ & $0.83^{b}$ & $0.42,1.24$ & -0.19 & $-0.60,0.22$ & 0.10 & $-0.30,0.51$ \\
\hline \multirow{2}{*}{ FSS by PSA } & Model $1^{c}$ & -0.12 & $-0.64,0.41$ & -0.04 & $-0.57,0.49$ & 0.07 & $-0.45,0.59$ \\
\hline & Model $2^{d}$ & -0.05 & $-0.56,0.46$ & -0.09 & $-0.62,0.45$ & 0.04 & $-0.48,0.57$ \\
\hline
\end{tabular}

Abbreviations: AES-C, Apathy Evaluation Scale Clinician-rated; FSS, Fatigue Severity Scale; MADRS, Montgomery-Åsberg Depression Rating Scale; PSA, post-stroke apathy; PSD, post-stroke depression; PSF, post-stroke fatigue; T0, 3 months post-stroke; T1, 6-month follow-up; T2, 12-month follow-up.

${ }^{a} p<0.05$.

${ }^{\mathrm{b}} \mathrm{p}<0.01$.

${ }^{c}$ Model 1: corrected for age, sex, highest level of education, and sleep apnea.

${ }^{d}$ Model 2: corrected for age, sex, highest level of education, sleep apnea, high neuroticism, and history of depression. 


\section{DISCUSSION}

This study aimed to disentangle the temporal relationship between PSF and PSD, and between PSF and PSA. Patients with PSF at baseline had higher depression scores that remained stable throughout time, and patients with PSD at baseline had higher fatigue scores that remained stable throughout time as well. No association between PSF and the baseline level or course of apathy symptoms was found, and neither in the reverse direction, as no association between PSA and the baseline level or course of fatigue symptoms was found. Additionally, change in fatigue score from baseline to 12-month follow-up was significantly associated with change in depression and change in apathy from baseline to 12-month follow-up. No associations were found when looking at change from baseline to 6-month follow-up.

Half of the stroke patients experienced PSF at baseline, which is in agreement with a previous meta-analysis that showed a range between 35\% and 92\%. ${ }^{1}$ PSD at baseline was present in almost $40 \%,{ }^{34}$ which is somewhat higher than the estimated prevalence of $31 \%$ reported in an earlier meta-analysis and baseline PSA was present in less than $20 \%$, which is lower than the reported estimated prevalence of $35 \%$ in the meta-analysis by van Dalen et al. ${ }^{15}$ However, this can be explained partially by the variability in the time range between the measurements and the stroke date, the cut-off scores that were applied, and the use of different questionnaires.

The present findings suggest that both PSF and PSD are frequent early after stroke and remain relatively stable throughout time. Half of the patients with PSF at baseline also had PSD, suggesting a strong bi-directional association between the two symptoms. These results are in agreement with the study of Lerdal et al..$^{35}$ who also found an association between early fatigue and early depressive symptoms. In addition, baseline depression scores were associated with high fatigue scores at follow-up. This is in line with a previous longitudinal study that suggested that baseline depression was associated with long-term fatigue. ${ }^{10}$

With respect to the temporal relationship between PSD and PSF, the results of this study indicate that fatigue and depression show a similar course over time and cooccur in a quarter of the stroke patients 3 months after stroke. Patients with PSD had high FSS scores, which remained stable over time, whereas patients with PSF had high MADRS scores that also remained stable over time. This suggests a bidirectional relationship, as the results also suggest that change in depression over time was associated with change in fatigue over time. 
Yet, some patients experience PSF in the absence of PSD, and vice versa, suggesting that PSD and PSF should be considered as distinct consequences of stroke. ${ }^{36}$ Early PSD, together with other factors, contributes to early PSF, and vice versa. According to the PSF model of Wu et al., ${ }^{6}$ potential factors that might be involved in this relationship include lesion location, inflammatory responses, personality traits, coping styles, and sleep apnea. These factors differ among individuals, which explains the inter-patient variability in the development of fatigue. ${ }^{1}$ Patient-tailored treatment is therefore important, as individual differences have to be taken into account when targeting PSD or PSF. With respect to treatment and rehabilitation programs, this suggests that an early focus on both PSD and PSF is important, as these conditions tend to persist. Since there are more treatment options available for depression, early screening for depression might have a positive effect on clinical outcome, ${ }^{37}$ and might also affect fatigue symptoms. Future longitudinal studies are needed to give more insight into the complex multifactorial origin of PSF. This requires large cohort studies taking into account the various factors that influence the complex relationship between PSD and PSF.

An important strength of the present study is the longitudinal design, which made it possible to study temporal associations. This is the first study that examined associations between fatigue and depression in both directions, and that also included the influence of apathy on this relationship. Well-validated instruments were used to measure fatigue, depression, and apathy and adequate statistical models for repeated measures were used to account for missing data. In addition, sleep apnea, neuroticism, and history of depression were included as they might influence the relationships between depression, apathy, and fatigue. Particularly the role of sleep apnea is important, as it has been shown to be a risk factor for incident stroke, ${ }^{38}$ and is associated with worse cognitive and functional status. ${ }^{39}$ Also in multiple sclerosis, ${ }^{40,41}$ associations between sleep apnea and fatigue have been found. Treatment of sleep apnea may have a beneficial effect on PSF, though future studies are needed to examine this. However, some limitations of the study should be noted. The exclusion of patients with severe aphasia and other neurological or psychiatric conditions made the study sample less representative, and it is reasonable to assume that stroke patients with a worse clinical outcome, who were unable to participate, have an even higher degree of PSF, PSD, or PSA, resulting in an underestimation of the presence of these conditions. 


\section{Chapter 4}

\section{CONCLUSIONS}

PSD and PSF were strongly associated throughout time, and the association seemed to be bi-directional. Although apathy scores were correlated with level of fatigue throughout time, this appeared largely to be due to co-morbid depression. The present results suggest that immediate screening for both PSD and PSF after stroke is important, as it might help to prevent the development of these symptoms at an early or later stage. This will result in better participation in rehabilitation programs, improving clinical outcome after stroke. 


\section{REFERENCES}

1. Duncan F, Wu S, Mead GE. Frequency and natural history of fatigue after stroke: a systematic review of longitudinal studies. J Psychosom Res. 2012;73:18-27.

2. Visser-Keizer AC, Hogenkamp A, Westerhof-Evers HJ, Egberink IJ, Spikman JM. Dutch Multifactor Fatigue Scale: A New Scale to Measure the Different Aspects of Fatigue After Acquired Brain Injury. Arch Phys Med Rehabil. 2015;96:1056-1063.

3. Christensen D, Johnsen SP, Watt T, Harder I, Kirkevold M, Andersen G. Dimensions of post-stroke fatigue: a two-year follow-up study. Cerebrovasc Dis. 2008;26:134-141.

4. Wondergem R, Pisters MF, Wouters EJ, Olthof N, de Bie RA, Visser-Meily JM, et al. The Course of Activities in Daily Living: Who Is at Risk for Decline after First Ever Stroke? Cerebrovasc Dis 2017;43:1-8.

5. van de Port IG, Kwakkel G, Schepers VP, Heinemans CT, Lindeman E. Is fatigue an independent factor associated with activities of daily living, instrumental activities of daily living and healthrelated quality of life in chronic stroke? Cerebrovasc Dis. 2006;23:40-45.

6. Wu S, Mead G, Macleod M, Chalder T. Model of understanding fatigue after stroke. Stroke 2015;46:893-898.

7. Naess H, Lunde L, Brogger J, Waje-Andreassen U. Fatigue among stroke patients on long-term follow-up. The Bergen Stroke Study. J Neurol Sci. 2012;312:138-41.

8. Ormstad H, Eilertsen G. A biopsychosocial model of fatigue and depression following stroke. Med Hypotheses 2015;85:835-841.

9. Wu S, Barugh A, Macleod M, Mead G. Psychological Associations of Poststroke Fatigue A Systematic Review and Meta-Analysis. Stroke 2014;45:1778-1783.

10. Passier P, Post M, van Zandvoort M, Rinkel G, Lindeman E, Visser-Meily J. Predicting fatigue 1 year after aneurysmal subarachnoid hemorrhage. J Neurol. 2011;258:1091-1097.

11. Snaphaan L, Van Der Werf S, de Leeuw FE. Time course and risk factors of post-stroke fatigue: a prospective cohort study. Eur J Neurol. 2011;18:611-617.

12. Lerdal A, Gay CL. Fatigue in the acute phase after first stroke predicts poorer physical health 18 months later. Neurology. 2013;81:1581-1587.

13. Naess $H$, Lunde L, Brogger J. The triad of pain, fatigue and depression in ischemic stroke patients: the Bergen Stroke Study. Cerebrovasc Dis. 2012;33(5):461-465.

14. Withall A, Brodaty H, Altendorf A, Sachdev PS. A longitudinal study examining the independence of apathy and depression after stroke: the Sydney Stroke Study. Int Psychogeriatr. 2011;23:264273.

15. van Dalen JW, van Charante EPM, Nederkoorn PJ, van Gool WA, Richard E. Poststroke apathy. Stroke 2013;44(3):851-60.

16. Caeiro L, Ferro JM, Costa J. Apathy secondary to stroke: a systematic review and meta-analysis. Cerebrovasc Dis. 2013;35(1):23-39.

17. Hackett ML, Köhler S, T O’Brien J, Mead GE. Neuropsychiatric outcomes of stroke. Lancet Neurol. 2014;13:525-534.

18. Marin RS. Differential Diagnosis and Classification of Apathy. Am J Psychiatry. 1990;147:22-30.

19. Douven E, Schievink SH, Verhey FR, van Oostenbrugge RJ, Aalten P, Staals J, et al. The Cognition and Affect after Stroke-a Prospective Evaluation of Risks (CASPER) study: rationale and design. BMC Neurol. 2016;16:1-11.

20. Jorm A. A short form of the Informant Questionnaire on Cognitive Decline in the Elderly (IQCODE): development and cross-validation. Psychol Med. 1994;24:145-153.

21. Folstein MF, Folstein SE, McHugh PR. "Mini-mental state": a practical method for grading the cognitive state of patients for the clinician. J Psychiatr Res. 1975;12:189-198.

22. Lees R, Stott DJ, Quinn TJ, Broomfield NM. Feasibility and diagnostic accuracy of early mood screening to diagnose persisting clinical depression/anxiety disorder after stroke. Cerebrovasc Dis. 2014;37(5):323-329. 
23. Krupp LB, LaRocca NG, Muir-Nash J, Steinberg AD. The fatigue severity scale: application to patients with multiple sclerosis and systemic lupus erythematosus. Arch Neurol. 1989;46:11211123.

24. Valko PO, Bassetti CL, Bloch KE, Held U, Baumann CR. Validation of the fatigue severity scale in a Swiss cohort. Sleep. 2008;31:1601-1607.

25. Choi-Kwon S, Ko M, Jun S-E, Kim J, Cho K-H, Nah H-W, et al. Post-Stroke Fatigue May Be Associated with the Promoter Region of a Monoamine Oxidase A Gene Polymorphism. Cerebrovasc Dis. 2016;43:54-58.

26. Marin RS, Biedrzycki RC, Firinciogullari S. Reliability and validity of the Apathy E7aluation Scale. Psychiatry Res. 1991;38:143-162.

27. Clarke DE, Ko JY, Kuhl EA, van Reekum R, Salvador R, Marin RS. Are the available apathy measures reliable and valid? A review of the psychometric evidence. J Psychosom Res. 2011;70:73-97.

28. Caeiro L, Ferro JM, Pinho e Melo T, Canhão P, Figueira ML. Post-stroke apathy: an exploratory longitudinal study. Cerebrovasc Dis. 2013;35(6):507-513.

29. Overbeek I, Schruers K, Griez E. Mini international neuropsychiatric interview: Nederlandse versie 5.0. 0. DSM-IV [Dutch version] Maastricht, The Netherlands: Universiteit Maastricht. 1999.

30. Montgomery SA, Åsberg M. A new depression scale designed to be sensitive to change. Br J Psychiatry. 1979;134:382-389.

31. Kang H-J, Stewart R, Kim J-M, Jang J-E, Kim S-Y, Bae K-Y, et al. Comparative validity of depression assessment scales for screening poststroke depression. J Affect Disord. 2013;147:186-191.

32. Mahoney FI. Functional evaluation: the Barthel index. Md State Med J. 1965;14:61-65.

33. Costa P, McCrae R. NEO five-factor inventory (NEO-FFI). Odessa, FL: Psychol Assess Resources. 1989.

34. Hackett ML, Pickles K. Part I: frequency of depression after stroke: an updated systematic review and meta-analysis of observational studies. Int J Stroke. 2014;9:1017-1025.

35. Lerdal A, Bakken LN, Rasmussen EF, Beiermann C, Ryen S, Pynten S, et al. Physical impairment, depressive symptoms and pre-stroke fatigue are related to fatigue in the acute phase after stroke. Disabil Rehabil. 2011;33:334-342.

36. Schepers VP, Visser-Meily AM, Ketelaar M, Lindeman E. Poststroke fatigue: course and its relation to personal and stroke-related factors. Arch Phys Med Rehabil. 2006;87:184-188.

37. Towfighi A, Ovbiagele B, El Husseini N, Hackett ML, Jorge RE, Kissela BM, et al. Poststroke Depression: A Scientific Statement for Healthcare Professionals From the American Heart Association/American Stroke Association. Stroke 2017;48:30-43.

38. Marshall NS, Wong KK, Cullen SR, Knuiman MW, Grunstein RR. Sleep apnea and 20-year followup for all-cause mortality, stroke, and cancer incidence and mortality in the Busselton Health Study cohort. J Clin Sleep Med. 2014;10(4):355-362.

39. Aaronson JA, van Bennekom CA, Hofman WF, van Bezeij T, van den Aardweg JG, Groet E, et al. Obstructive sleep apnea is related to impaired cognitive and functional status after stroke. Sleep. 2015;38(9):1431-1437.

40. Kaminska M, Kimoff R, Benedetti A, Robinson A, Bar-Or A, Lapierre Y, et al. Obstructive sleep apnea is associated with fatigue in multiple sclerosis. Mult Scler. 2012;18(8):1159-1169.

41. Braley TJ, Segal BM, Chervin RD. Obstructive sleep apnea and fatigue in patients with multiple sclerosis. J Clin Sleep Med. 2014;10(2):155-162. 


\section{SUPPLEMENTAL DATA}

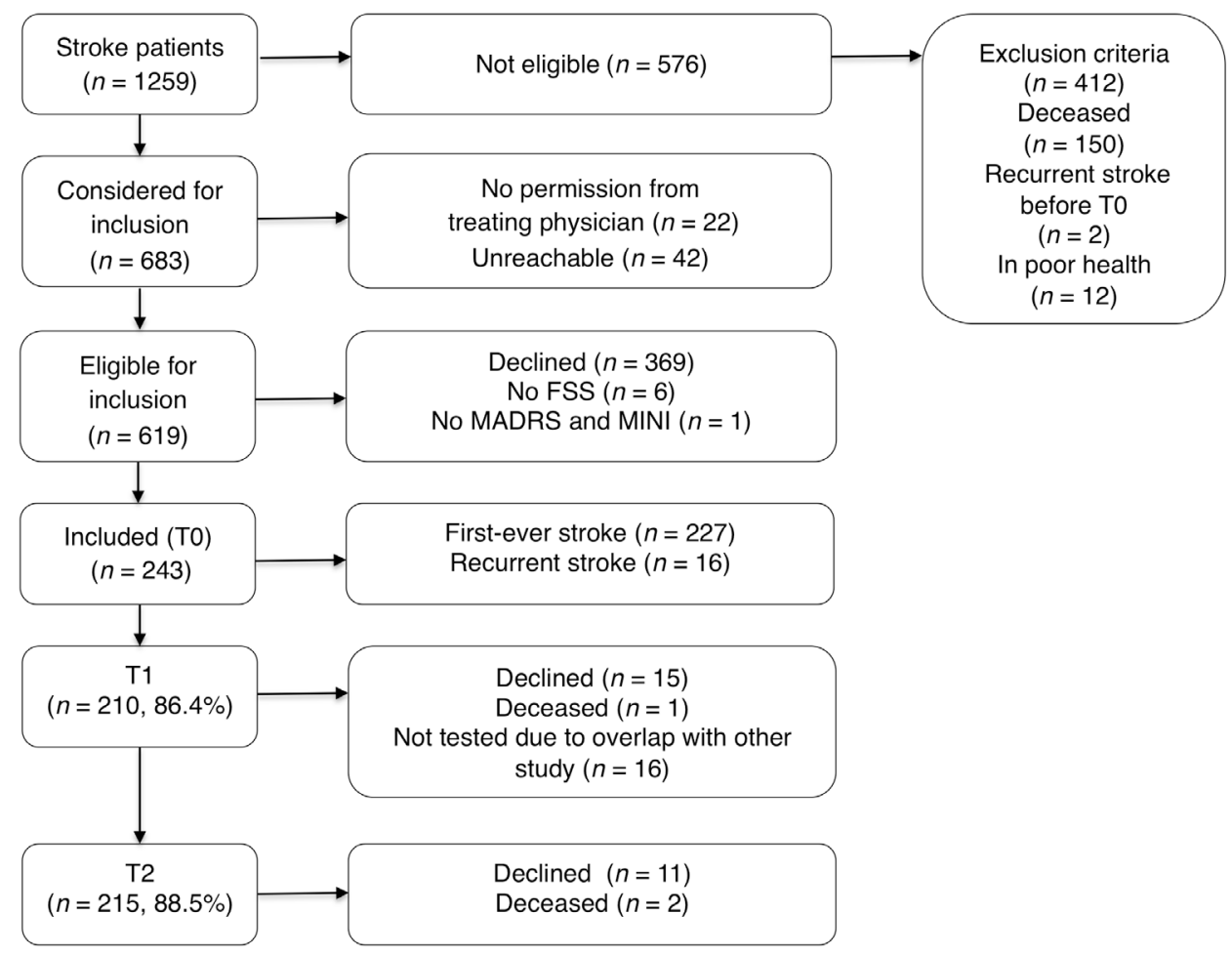

Supplementary Figure 1. Flowchart of inclusion process Note: Twenty patients who did not participate at T1, agreed to participate at T2. Abbreviations: FSS, Fatigue Severity Scale; MADRS, Montgomery-Åsberg Depression Rating Scale; MINI, Mini International Neuropsychiatric Interview; T0, baseline measurement 3 months post stroke; T1, follow-up 9 months post-stroke; T2, follow-up 15 months post-stroke. 


\section{Chapter 4}

Supplementary Table 1. Baseline characteristics for total sample, and separate for patients who completed all measurements and patients who dropped out at 1 or 2 time points

\begin{tabular}{|c|c|c|c|}
\hline Variable & $\begin{array}{l}\text { All patients T0 } \\
\qquad(n=243)\end{array}$ & $\begin{array}{l}\text { Complete follow-up } \\
\qquad(n=216)\end{array}$ & $\begin{array}{l}\text { Dropouts } \\
(n=27)\end{array}$ \\
\hline Age (in years) & $67.41(11.76)$ & $66.91(11.65)$ & $71.45(12.06)$ \\
\hline Male sex, $n(\%)$ & $157(64.6)$ & $142(65.7)$ & $15(55.6)$ \\
\hline Low education, $n(\%)$ & $100(41.3)$ & $86(39.8)$ & $14(53.9)$ \\
\hline Middle education, $n(\%)$ & $85(35.1)$ & $77(35.7)$ & $8(30.8)$ \\
\hline High education, $n(\%)$ & $57(23.6)$ & $53(24.5)$ & $4(15.4)$ \\
\hline Time since stroke (months) & $2.93(0.43)$ & $2.92(0.43)$ & $2.99(0.43)$ \\
\hline First-ever stroke, $n(\%)$ & $227(93.4)$ & $202(93.5)$ & $25(92.6)$ \\
\hline Barthel Index & $19.44(1.47)$ & $19.47(1.47)$ & $19.22(1.50)$ \\
\hline Sleep apnea, $n(\%)$ & $18(7.4)$ & $17(7.9)$ & $1(3.7)$ \\
\hline High neuroticism, $n(\%)$ & $115(48.7)$ & $102(48.1)$ & $13(54.2)$ \\
\hline Pre-stroke depression, $n(\%)$ & $55(22.6)$ & $47(21.8)$ & $8(29.6)$ \\
\hline Family history of depression, $n(\%)$ & $34(14.1)$ & $33(15.3)$ & $1(3.9)$ \\
\hline MADRS & $6.07(5.84)$ & $6.30(5.94)$ & $4.30(4.68)$ \\
\hline$A E S-C$ & $26.36(7.87)$ & $26.46(8.04)$ & $25.56(6.42)$ \\
\hline FSS & $3.78(1.59)$ & $3.79(1.56)$ & $3.68(1.80)$ \\
\hline MINI minor or major depression, $n(\%)$ & $28(11.5)$ & $26(12.0)$ & $2(7.4)$ \\
\hline MDD & $13(5.4)$ & $12(5.6)$ & $1(3.7)$ \\
\hline MIND & $15(6.2)$ & $14(6.5)$ & $1(3.7)$ \\
\hline PSA, $n(\%)$ & $40(16.5)$ & $37(17.1)$ & $3(11.1)$ \\
\hline$P S D, n(\%)$ & $94(38.7)$ & $87(40.3)$ & $7(25.9)$ \\
\hline PSF, $n(\%)$ & $119(49.0)$ & $106(49.1)$ & $13(48.2)$ \\
\hline
\end{tabular}

Abbreviations: AES-C, Apathy Evaluation Scale Clinician-rated; FSS, Fatigue Severity Scale; MADRS, Montgomery-Äsberg Depression Rating Scale; MDD, major depressive disorder; MIND, minor depression; MINI, Mini International Neuropsychiatric Interview; PSA, post-stroke apathy; PSD, poststroke depression; T0, baseline measurement 3 months post stroke. Data area mean (SD), unless otherwise specified.

Note: ${ }^{\mathrm{a}} p<0.05$ compared to complete follow-up, ${ }^{\mathrm{b}} p<0.01$ compared to complete follow-up. 


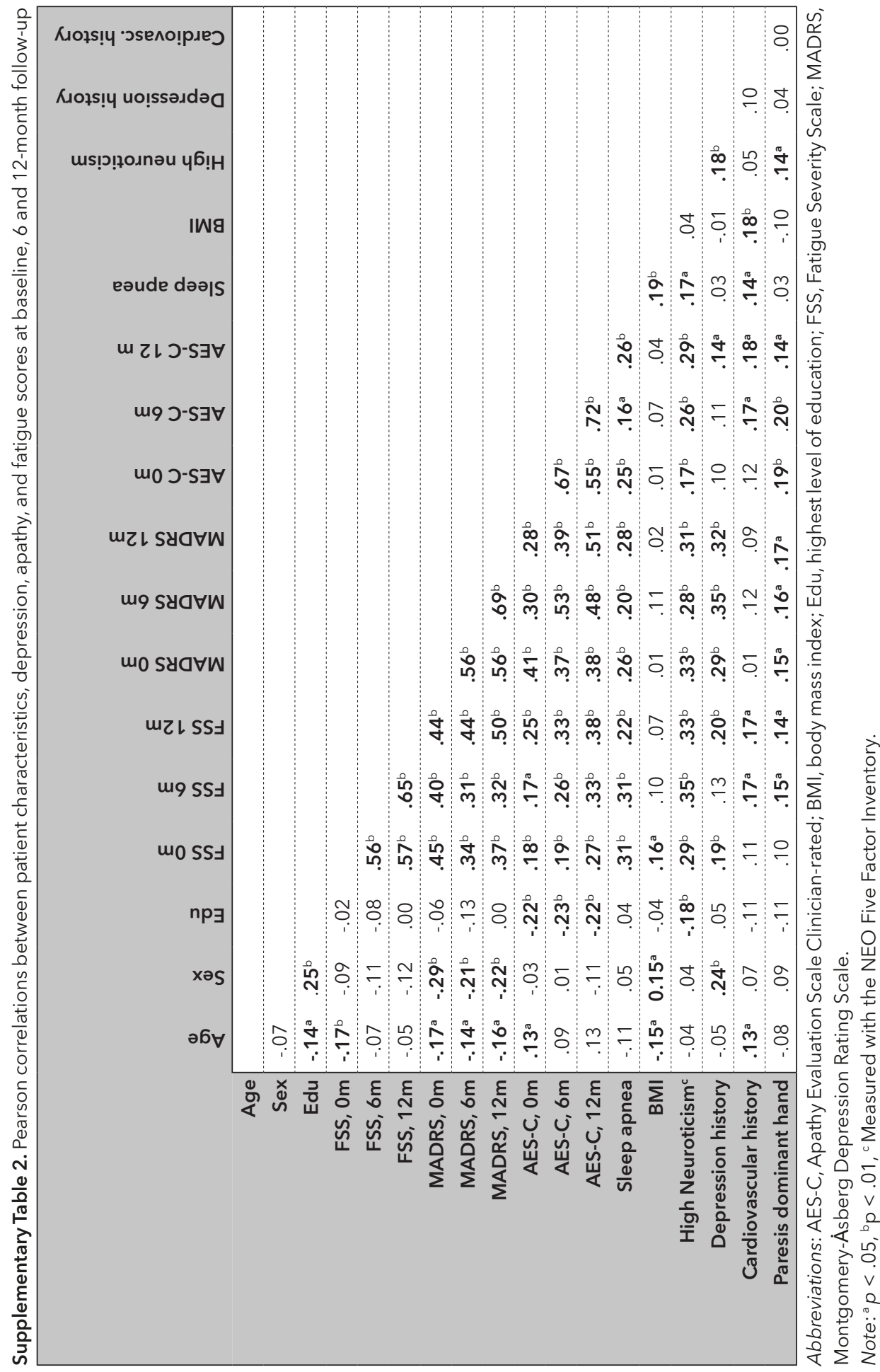




\section{CHAPTER 5}




\section{PREMORBID PERSONALITY AND COURSE OF SYMPTOMS OF DEPRESSION AND APATHY AFTER STROKE: RESULTS OF THE CASPER STUDY}

\section{Elles Douven}

Julie Staals

Syenna HJ Schievink

Robert J van Oostenbrugge

Frans RJ Verhey

Sascha Wetzels-Meertens

Sebastian Köhler

Pauline Aalten 


\section{ABSTRACT}

Objective: Post-stroke depression (PSD) and post-stroke apathy (PSA) are both associated with adverse outcome after stroke. This study aimed to examine whether pre-morbid personality traits predict the course of PSD and PSA over 12 months.

Methods: In this prospective cohort study, 241 stroke patients completed the NEO Five Factor Inventory, Montgomery-Åsberg Depression Rating Scale, and Apathy Evaluation Scale at 3 months post-stroke. Neuropsychiatric assessment was repeated at 6 - and 12-month follow-up after initial testing.

Results: Linear mixed models showed that high neuroticism was associated with higher levels of PSD at baseline, which remained stable at follow-up. Low extraversion was associated with higher levels of PSA at baseline, which also remained stable throughout time. Similarly, low openness and conscientiousness were associated with higher levels of PSA at baseline, which remained high at 6-month follow-up, but decreased at 12-month follow-up. None of the personality traits showed a significant overall groupby-time interaction.

Conclusion: Personality traits are associated with the development and sustainability of PSD and PSA. The traits associated with PSD and PSA were different, providing support for the independence of these constructs. The findings highlight the importance to take personality traits into account as a potential vulnerability factor for PSD and PSA. 


\section{INTRODUCTION}

Apathy and depression are common neuropsychiatric consequences after stroke, both showing prevalence rates of around 30\%., ${ }^{1,2}$ Post-stroke depression (PSD) is known to have a negative impact on the effectiveness of rehabilitation treatment, ${ }^{3}$ whereas post-stroke apathy (PSA) is associated with poor functional recovery. ${ }^{4,5}$ Moreover, both reduce quality of life after stroke., ${ }^{2,6-8}$ While previous research on determinants of PSD and PSA focused mainly on stroke-related factors, such as lesion characteristics, ${ }^{9-11}$ psychological factors, including personality traits, may play a role as well.

Personality traits are relatively stable manners of feeling, thinking, and acting of individuals ${ }^{12}$ and according to the five-factor model, ${ }^{13}$ personality can be represented by a five-factor trait structure, comprising neuroticism, extraversion, openness to experience, agreeableness, and conscientiousness. Previous studies in the general population found personality traits, and particularly neuroticism, to be an important predictor of onset and course of neuropsychiatric symptoms, ${ }^{14-17}$ but studies on personality factors in a stroke population are scarce. Some previous cross-sectional studies have reported an association between neuroticism and PSD, ${ }^{18,19}$ particularly in the acute stroke phase. ${ }^{19,20} \mathrm{~A}$ single prospective study has shown that high neuroticism was an important predictor of 1 -year PSD, ${ }^{3}$ but no evidence was found for associations with any of the other five-factor personality traits. For PSA, the relationship with premorbid personality traits has only been examined in one study, but no association was found. ${ }^{21}$

Information on premorbid personality could be helpful to detect patients at risk for developing neuropsychiatric symptoms in patients recovering from stroke. Previous cross-sectional studies already indicated a strong link between neuroticism and PSD. However, as previously pointed out by Aben et al., ${ }^{3}$ the association between personality traits and neuropsychiatric symptoms after stroke should preferably be studied in large longitudinal cohorts with multiple time points, assessing all five-factor personality traits. PSD and PSA are independent though overlapping syndromes, ${ }^{22,23}$ and the studies examining personality traits in association with PSD did not control for this overlap in symptomatology between the two syndromes, which could have biased the results. Therefore, the present study followed a cohort of 250 stroke patients to assess whether pre-morbid personality traits predict the course of PSD and PSA over 12 months. 


\section{METHODS}

\section{STUDY POPULATION}

Participants of this study took part in the Cognition and Affect - a Prospective Evaluation of Risks (CASPER) study, a prospective clinical cohort study into predictors of cognitive and neuropsychiatric disorders after stroke. The study was approved by the Medical Ethics Committee of Maastricht University Medical Center (MUMC+).

We included patients who suffered from a non-fatal ischemic or hemorrhagic stroke who were admitted to the Stroke Unit of MUMC+ or Zuyderland Medical Center in Sittard and Heerlen (the Netherlands) between June 2013 and August 2015. Stroke was defined as a clinical stroke syndrome, with sudden neurological dysfunction lasting $>24$ hours, with no apparent cause other than that of vascular origin. Ischemic strokes could be cortical or lacunar, and hemorrhagic strokes were non-traumatic deep, lobar, cerebellar, or brainstem hemorrhages as evidenced by a clinical brain scan (for details see Douven et al. ${ }^{24}$ ). Exclusion criteria include age < 40 years, pre-stroke dementia, pre-existing cognitive impairment, mental retardation, a Mini-Mental State Examination Score $<15,{ }^{25}$ neurological or psychiatric diseases other than depression that are known to affect cognition, insufficient knowledge of the Dutch language, too severe aphasia to understand the study procedure, an Informant Questionnaire on Cognitive Decline in the Elderly score $3.60,{ }^{26}$ no written informed consent, blindness, history of stroke $<3$ years or residual symptoms from previous stroke, and post-surgery stroke / postanoxic encephalopathy.

\section{PROCEDURE}

Baseline measurements (TO) were scheduled approximately 3 months after stroke (median $=2.9$ months, interquartile range $=2.0-4.3$ ), to avoid interference with acute care and rehabilitation. Socio-demographic characteristics were recorded, and neuropsychiatric questionnaires were administered to the patient, in which the presence of depression and apathy was assessed. The neuropsychiatric interview was repeated in the chronic stroke phase ( 9 and 15 months post-stroke; T1 and T2, respectively). Pre-morbid personality was only assessed at T0, as it was assumed that personality traits remain stable over time. 


\section{CLINICAL MEASURES}

Personality was assessed with the NEO Five-Factor Inventory (NEO-FFI). ${ }^{27,28}$ This self-report questionnaire consists of 60 statements covering the 5 personality traits extraversion, neuroticism, openness to new experiences, agreeableness, and conscientiousness. Each statement was rated by the participant on a 5-point scale ranging from "strongly disagree" to "strongly agree". For each personality trait a total dimension score between 12 and 60 was obtained. A median split was applied on all personality domains to dichotomize the personality traits in low versus high premorbid personality domains. ${ }^{29}$

The severity of PSD symptoms was measured with the Montgomery-Åsberg Depression Rating Scale (MADRS), a 10-item clinician-rated scale designed to be especially sensitive to change in symptom severity. ${ }^{30}$ Each item was scored from 0 to 6, resulting in a total score between 0 and 60, with higher scores reflecting more severe symptoms of depression. The MADRS was used as outcome measure of PSD symptoms as it focuses less on somatic symptoms, and is therefore evaluated as a valid instrument for measuring the severity of depressive symptoms after stroke. ${ }^{31}$ In addition, the Mini International Neuropsychiatric Inventory (MINI), a semi-structured interview based on DSM-IV criteria, was administered for the diagnosis major depressive disorder (MDD) or minor depression (MIND) by a trained research (neuro)psychologist. ${ }^{32}$

The Apathy Evaluation Scale (AES) was used to measure the presence and severity of PSA. ${ }^{33}$ It consists of 18 items rated on a 4-point scale, resulting in a total score between 18 and 72, with higher scores representing a higher level of apathy symptoms. The clinician-rated version of the AES was used, as it has been rated as the most valid apathy instrument. ${ }^{34}$ A cut-off score of $\geq 37$ was applied to define clinically relevant apathy. ${ }^{22,35}$ The Barthel Index was used as a measure of impairment in activities of daily living. ${ }^{36}$

\section{STATISTICAL ANALYSIS}

All statistical analyses were performed with Stata version 13.1 for Mac OS X (StataCorp, USA). An alpha level of .05 (two-sided) was used for all analyses. Differences in demographic variables between patients who completed all measurements and patients who dropped out at 1 or 2 time points were tested using $X^{2}$ tests for qualitative variables and $t$-tests for quantitative variables. Separate linear mixed models were performed to measure the effect of personality traits on the course of depression (MADRS score) or apathy (AES-C score) from T0 to T2 as outcome measure. A group (personality trait: low, high) by time (3-levels: T0, T1, T2) interaction was included to study differences in the course of depression or apathy 
between the groups. The models included a random intercept and random slope with an unstructured correlation matrix as this resulted in the best fit according to likelihood ratio testing. The analyses were corrected for age, sex, highest level of education, history of depression, and Barthel Index score, as based on previous studies these factors seem to influence the association between personality traits and PSD, ${ }^{3}$ and probably also PSA. Additionally, we also added apathy score to the model to correct for a possible confounding effect of apathy on depression, and vice versa, because of a possible overlap in symptomatology.

\section{RESULTS}

Of the 619 eligible patients, 250 patients agreed to participate and signed informed consent. Refusers were more often female (53.4\% versus $35.6 \%$; $p<$ $.001)$ and older than participants $(73.2 \pm 12.3$ versus $67.3 \pm 11.8$ years, $p<.001)$. Of the 250 participants, 241 (96\%) completed the NEO-FFI. Of them, $211(87.2 \%)$ completed T1 (Supplementary Figure 1) and another 12 patients dropped-out at T2 (10 refused, 2 deceased). Eighteen of the patients who did not participate at T1 re-entered the study at T2, which resulted in 217 (89.7\%) patients who completed T2. No differences were found between the 22 patients (9.1\%) lost to follow-up at 1 or 2 time points and the 219 patients (90.9\%) who completed all measurements (Supplementary Table 1).

Table 1 shows the baseline characteristics of the total sample. At T0, 26 patients $(10.8 \%)$ met DSM-IV criteria for either MDD $(n=13)$ or MIND $(n=13)$. A MINI depression diagnosis (MDD or MIND) was found in $14.2 \%$ at T1, and in $11.1 \%$ at T2. The average MADRS score at T0 was $6.1(S D=5.8)$ in the total sample, 15.9 $(S D=7.4)$ in patients with a MINI depression diagnosis and $4.9(S D=4.3)$ for nondepressed patients $(t(230)=-11.02, p<.001)$. Mean MADRS scores in the total cohort remained stable at T1 $(6.8, S D=7.2)$ and T2 (6.6, SD = 7.5). Average AES-C scores were $26.4(S D=7.9)$ at $\mathrm{T} 0,28.4(S D=9.0)$ at T1 and $28.1(S D=9.4)$ at T2. Twenty-four patients (10.0\%) scored above the cut-off on the AES-C at T0. This frequency increased to $19.9 \%$ at T1 and decreased to $17.1 \%$ at T2.

\section{PERSONALITY TRAITS AND POST-STROKE DEPRESSIVE SYMPTOMS}

Table 2 gives the results of the linear mixed models examining differences in the course of depression between patients with a high and a low score on each personality trait. Significantly higher depression scores at T0 were found in patients with high neuroticism $\left(X^{2}=13.31\right.$; $\left.d f=1 ; p<0.001\right)$. This group effect remained significant at T1 $(p=.011)$ and T2 $(p=.002$; Figure 1). No significant 
group difference in depression score was found at T0 between patients with high and low scores on agreeableness, openness, extraversion, or conscientiousness, respectively. None of the personality traits showed a significant overall groupby-time interaction. Also, no significant change between individual time points (baseline to T1, or baseline to T2) was found (Table 2), implying there were no significant differences in the course of depressive symptoms over time.

Table 1. Baseline characteristics of patients

\begin{tabular}{|c|c|}
\hline Variable & Total $(n=241)$ \\
\hline Age in years (SD) & $67.31(11.78)$ \\
\hline Male sex, $n(\%)$ & $158(65.6)$ \\
\hline Low education, $n(\%)$ & $97(40.4)$ \\
\hline Middle education, $n(\%)$ & $84(35.0)$ \\
\hline High education, $n(\%)$ & $59(24.6)$ \\
\hline First-ever stroke, $n(\%)$ & $225(93.4)$ \\
\hline Barthel score (SD) & $19.44(1.49)$ \\
\hline \multicolumn{2}{|l|}{ Personality score (SD) } \\
\hline Neuroticism & $31.60(4.89)$ \\
\hline Agreeableness & $36.54(4.60)$ \\
\hline Openness & $36.85(4.10)$ \\
\hline Extraversion & $38.25(4.19)$ \\
\hline Conscientiousness & $39.63(3.75)$ \\
\hline History of depression, $n(\%)$ & $55(22.8)$ \\
\hline Family history of depression, $n(\%)$ & $34(14.2)$ \\
\hline MADRS score (SD) & $6.06(5.84)$ \\
\hline AES-C score (SD) & $26.41(7.86)$ \\
\hline MINI minor / major depression, $n(\%)$ & $26(10.8)$ \\
\hline $\mathrm{MDD}, n(\%)$ & $13(5.4)$ \\
\hline MIND, $n(\%)$ & $13(5.4)$ \\
\hline PSA, $n(\%)$ & $24(10.0)$ \\
\hline
\end{tabular}

Abbreviations: AES-C, Apathy Evaluation Scale Clinician-rated; MADRS, Montgomery-Åsberg Depression Rating Scale; MDD, major depressive disorder; MIND, minor depression; MINI Mini International Neuropsychiatric Interview; PSA, post-stroke apathy. 


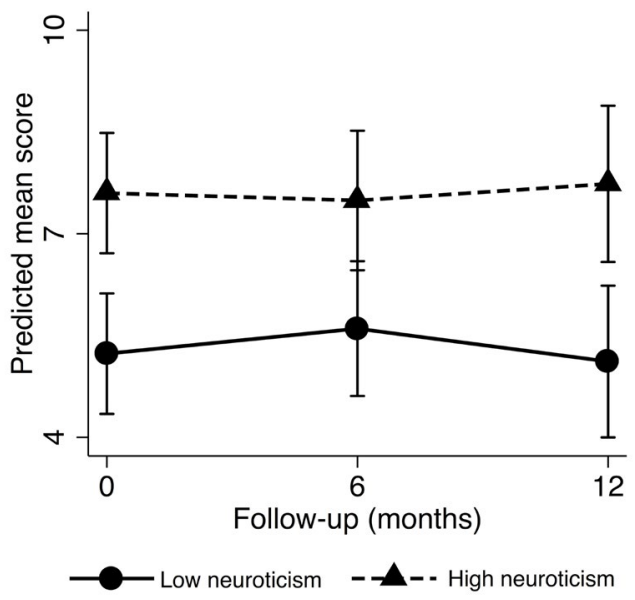

Figure 1. Course of depressive symptoms by neuroticism group

Note: Based on random-effects analysis with random intercept, random slope and unstructured correlation matrix, adjusted for age at baseline, sex, level of education, Barthel Index, history of depression, and apathy score. Predicted mean scores are estimated marginal means for neuroticism group by time, with all covariates fixed at their means. Higher mean scores indicate a higher level of depression.

Table 2. Differences in baseline depression and apathy and change in depression and apathy by personality trait

\begin{tabular}{|c|c|c|c|c|c|c|c|}
\hline \multirow{3}{*}{ Parameter } & \multicolumn{7}{|c|}{ Time } \\
\hline & \multicolumn{2}{|c|}{ Baseline } & \multicolumn{2}{|c|}{$\begin{array}{l}\text { 6-month } \\
\text { follow-up }\end{array}$} & \multicolumn{2}{|c|}{$\begin{array}{l}\text { 12-month } \\
\text { follow-up }\end{array}$} & \multirow{2}{*}{$\begin{array}{c}\begin{array}{c}\text { Personality } \\
\text { by Time }\end{array} \\
X^{2}(2 d f) \\
\end{array}$} \\
\hline & Difference & $95 \% \mathrm{Cl}$ & Change & $95 \% \mathrm{Cl}$ & Change & $95 \% \mathrm{Cl}$ & \\
\hline \multicolumn{8}{|l|}{ MADRS score by } \\
\hline Neuroticism & $2.37^{b}$ & $1.10,3.64$ & -0.48 & $-2.02,1.06$ & 0.25 & $-1.39,1.90$ & 0.89 \\
\hline Agreeableness & 0.60 & $-0.71,1.91$ & 0.66 & $-0.88,2.20$ & 1.25 & $-0.40,2.89$ & 2.22 \\
\hline Openness & -0.61 & $-1.90,0.68$ & 0.03 & $-1.51,1.58$ & -0.56 & $-2.22,1.10$ & 0.64 \\
\hline Extraversion & 0.25 & $-1.08,1.58$ & -0.48 & $-2.03,1.07$ & -0.73 & $-2.40,0.93$ & 0.78 \\
\hline Conscientiousness & 0.44 & $-0.84,1.71$ & 0.48 & $-1.05,2.02$ & 0.20 & $-1.45,1.85$ & 0.39 \\
\hline \multicolumn{8}{|l|}{ AES-C score by } \\
\hline Neuroticism & 0.23 & $-1.64,2.11$ & 1.61 & $-0.17,3.39$ & 1.93 & $-0.12,3.97$ & 4.22 \\
\hline Agreeableness & 1.61 & $-0.24,3.47$ & 1.08 & $-0.71,2.87$ & -0.29 & $-2.39,1.81$ & 2.73 \\
\hline Openness & -1.81 & $-3.62,0.00$ & -0.66 & $-2.45,1.14$ & -0.03 & $-2.15,2.08$ & 0.73 \\
\hline Extraversion & $-4.57^{b}$ & $-6.33,-2.80$ & 0.32 & $-1.48,2.13$ & 0.14 & $-1.97,2.25$ & 0.13 \\
\hline Conscientiousness & $-2.06^{a}$ & $-3.86,-0.27$ & -0.21 & $-2.01,1.58$ & 0.37 & $-1.73,2.47$ & 0.39 \\
\hline
\end{tabular}

Model: personality trait, time, personality by time, sex, age at baseline, education, Barthel score at baseline, and history of depression. Analyses with MADRS score as outcome were additionally corrected for AES-C score, and vice versa. ${ }^{a} p<0.05,{ }^{b} p<0.01,{ }^{c}$ Overall interaction between personality trait (low, high) and time (baseline, 6-month follow-up, 12-month follow-up, as indicated by $\mathrm{X}^{2}, d f=2$ ). Abbreviations: AES-C, Apathy Evaluation Scale Clinician-rated; $\mathrm{Cl}$, confidence interval; MADRS, Montgomery-Åsberg Depression Rating Scale. 

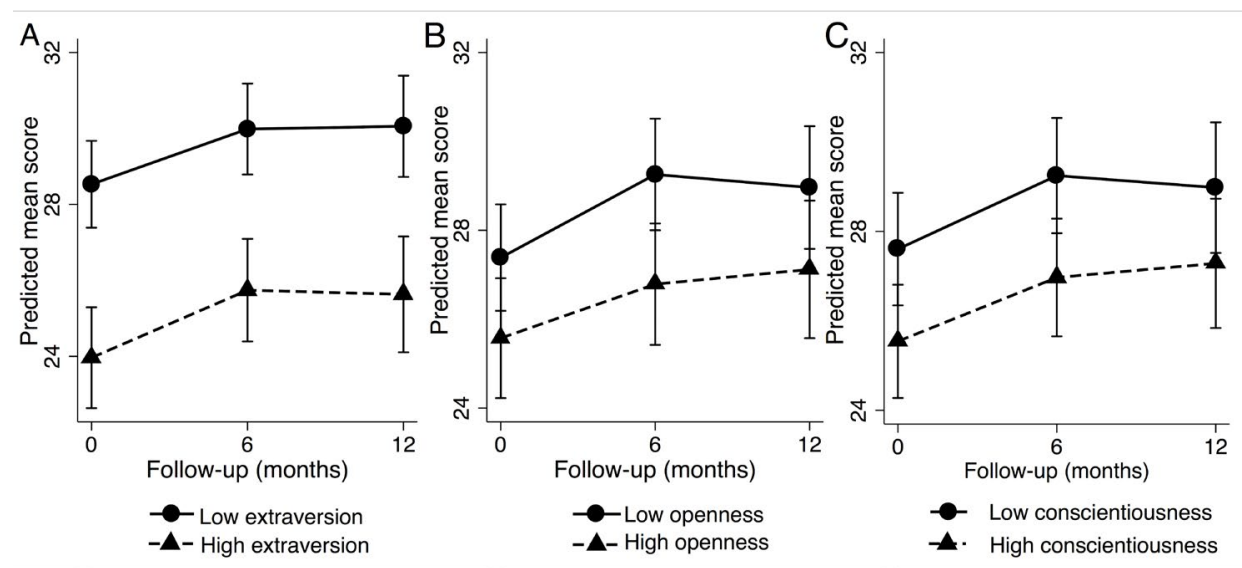

Figure 2. Course of apathy by extraversion, openness, and conscientiousness group

A, course of apathy scores by extraversion group. B, course of apathy scores by openness group. C, course of apathy scores by conscientiousness group. Note: Based on random-effects analysis with random intercept, random slope and unstructured correlation matrix, adjusted for age at baseline, sex, level of education, Barthel Index, history of depression, and depression score. Predicted mean scores are estimated marginal means for personality trait group by time, with all covariates fixed at their means. Higher mean scores indicate a higher level of apathy.

\section{PERSONALITY TRAITS AND POST-STROKE APATHY SYMPTOMS}

Significantly higher apathy scores were found in patients with low extraversion at T0 $\left(X^{2}=25.66 ; d f=1 ; p<0.001\right)$, which remained stable at T1 $(p<0.001$; Figure $2 A)$ and T2 $(p<0.001)$. Apathy scores were almost significantly higher in patients with low openness at T0 $\left(X^{2}=3.83 ; d f=1 ; p=0.050\right)$, which became significant at T1 ( $p=0.010$, Figure $2 B$ ), but not at T2. Similarly, significantly higher apathy scores were found in patients with low conscientiousness at T0 $\left(X^{2}=5.08 ; d f=\right.$ 1 ; $p=0.024)$, which remained stable at T1 $(p=0.016)$, but not at T2 (Figure $2 C$ ). With respect to neuroticism and agreeableness, no significant group difference in apathy score was found at T0. None of the personality traits showed a significant overall group-by-time interaction. Also, no significant change between individual time points (baseline to T1, or baseline to T2) was found (Table 2), implying there were no significant differences in change of apathy over time. 


\section{DISCUSSION}

This study investigated whether pre-morbid personality traits are a predictive factor for the development and course of depressive or apathetic symptoms after stroke. The main finding of this study was a consistent positive relationship between PSD and neuroticism over the course of 12 months, whereas there was a consistent negative relationship between PSA and extraversion. Furthermore, a negative association between PSA and openness and conscientiousness was found at baseline and 6-month follow-up, but not at 12-month follow-up. None of the personality traits showed a significant overall group-by-time interaction.

Our finding that individuals who score high on neuroticism are more likely to develop depressive symptoms post-stroke is in agreement with the results of the 1-year longitudinal study of Aben et al. ${ }^{3}$ and the cross-sectional study of Hwang et al. ${ }^{18}$ The present study adds that neuroticism is a prognostic factor in determining the course of PSD symptoms over time, i.e. from the post-acute to the chronic stroke phase. In contrast with earlier studies on neuroticism and depression, ${ }^{3,18}$ we also controlled for level of apathy, as it might influence this relationship. Research has shown that social inhibition ${ }^{37}$ and maladaptive forms of emotion regulation, ${ }^{38}$ which may both be associated with apathy, ${ }^{39,40}$ mediate the relationship between neuroticism and depression in elderly. Emotion regulatory processes can be affected by stroke, which can have a negative influence on social participation. ${ }^{41}$ Therefore, also in stroke patients it may be of importance to look at the specific effect of neuroticism on PSD, independent of PSA. In contrast to our findings, Van Mierlo et al. ${ }^{41}$ reported that extraversion was associated with PSD at 2 months post-stroke, too, which might be explained by the fact that they did not consider the role of PSA in this association.

For PSA, individuals who scored high on extraversion were less likely to develop apathetic symptoms post-stroke. This finding has not been examined in a stroke population before, but it is consistent with a cross-sectional study including 286 patients with neurodegenerative diseases that showed a negative association between apathy scores and extraversion. ${ }^{42}$ Furthermore, low conscientiousness and low openness were associated with high apathy levels at baseline in our study, which remained stable at 6-month follow-up. Also, these associations have not been studied before in a stroke population, though the results were consistent with findings in a sample with mild cognitive impairment, in which retrospective change in conscientiousness from 5 years prior to assessment to baseline was positively associated with apathy, and premorbid openness was negatively associated with apathy. ${ }^{43}$ 
Some controversy still exists whether depression and apathy are different, yet overlapping, disease entities. Our finding that personality traits are differentially associated with PSD and PSA suggests they are independent, at least in a stroke population. ${ }^{23,44}$ Furthermore, our results are in agreement with the hypothesis that, besides an important role for biological factors, ${ }^{45}$ psychological factors also play a role in the development of $\mathrm{PSD}^{46}$ and the same theory seems to be true for PSA. Hence, future studies should focus on how personality traits and other psychological factors alone and in interaction with biological factors predict the development and course of PSD and PSA. This is important for treatment and rehabilitation, as focusing on early detection of PSD and PSA might result in a better clinical outcome after stroke. ${ }^{47}$

Strengths of this study are the longitudinal design and the low dropout rates at follow-up. In addition, validated instruments were used to evaluate the presence and level of depression, apathy, and personality traits. The consideration of the role of all five-factor personality traits in the development of both depression and apathy provides a more complete picture of the role premorbid personality plays in neuropsychiatric symptoms after stroke.

There are also some limitations that should be appointed. First, personality is assumed to be relatively stable over time, and studies have confirmed this partly, ${ }^{12}$ but personality traits were measured after stroke. Hence, we do not know whether and how the stroke event itself had an influence on the self-rating of pre-morbid personality, so the results may possibly be not completely representative for 'premorbid' personality. An additional informant rating of premorbid personality could have been valuable, as the informant can indicate whether a trait was already high or low before the stroke and the informant rating is not influenced by the current mood state of the patient. However, due to time constraints and because not every participant would have an informant we only collected self-rated premorbid personality, which has been commonly used in previous stroke studies. ${ }^{3,18,20}$ Nevertheless, future studies should take this critical aspect into account, suggesting to assess (premorbid) personality in both the stroke patient and their primary caregiver or informant. Second, no significant main effect of an interaction between personality traits and time was found. Therefore, the individual traits by time point analyses are of exploratory nature and should be treated very carefully for further interpretation. We still include them for comparison with previous studies that looked at individual time points only. Third, the exclusion of patients with aphasia, pre-stroke dementia, and co-morbid (neurological) conditions (e.g. Parkinson's disease, epilepsy, substance abuse) resulted in a less representative stroke sample. Lastly, patients with depressive or apathetic symptoms at an early stage might have been less motivated to participate in the study in the first 
place, which could have resulted in an underestimation of depression and apathy symptoms in our cohort, as the prevalence of PSA and PSD at baseline in our cohort were lower than the pooled estimated prevalences in previous meta-analyses. ${ }^{1,2}$ In addition, patients with a severe stroke might be underrepresented in our sample, as is common in these kind of studies. This might have resulted in lower levels of PSA and PSD in our sample as well, although a previous study showed a similar frequency of PSD in minor stroke..$^{48}$

\section{CONCLUSIONS}

Personality traits are associated with the development and sustainability of PSD or PSA. The traits associated with PSD and PSA were different, providing support for the independence of these constructs. The findings highlight the importance to take personality traits into account as a potential vulnerability factor for PSD and PSA. 


\section{REFERENCES}

1. Hackett ML, Pickles K. Part I: frequency of depression after stroke: an updated systematic review and meta-analysis of observational studies. Int J Stroke. 2014;9:1017-1025.

2. van Dalen JW, van Charante EPM, Nederkoorn PJ, van Gool WA, Richard E. Poststroke apathy. Stroke. 2013;44:851-860.

3. Aben I, Denollet J, Lousberg R, Verhey F, Wojciechowski F, Honig A. Personality and Vulnerability to Depression in Stroke Patients A 1-Year Prospective Follow-Up Study. Stroke. 2002;33:23912395.

4. Hama S, Yamashita H, Shigenobu M, Watanabe A, Hiramoto K, Kurisu K, et al. Depression or apathy and functional recovery after stroke. Int J Geriatr Psych. 2007;22:1046-1051.

5. Santa N, Sugimori H, Kusuda K, Yamashita Y, Ibayashi S, lida M. Apathy and functional recovery following first-ever stroke. Int J Rehabil Res. 2008;31:321-326.

6. Carod-Artal J, Egido JA, González JL, De Seijas EV. Quality of life among stroke survivors evaluated 1 year after stroke. Stroke. 2000;31:2995-3000.

7. Kouwenhoven SE, Kirkevold M, Engedal K, Kim HS. Depression in acute stroke: prevalence, dominant symptoms and associated factors. A systematic literature review. Disabil Rehabil. 2011;33:539-556.

8. Mayo NE, Fellows LK, Scott SC, Cameron J, Wood-Dauphinee S. A longitudinal view of apathy and its impact after stroke. Stroke. 2009;40:3299-3307.

9. Robinson RG, Starkstein SE. Mood disorders following stroke: New findings and future directions. J Geriatr Psychiat. 1989;22:1-15.

10. Robinson RG. The clinical neuropsychiatry of stroke: cognitive, behavioral and emotional disorders following vascular brain injury. Cambridge University Press; 1998.

11. Wei N, Yong W, Li X, Zhou Y, Deng M, Zhu H, et al. Post-stroke depression and lesion location: a systematic review. J Neurol. 2015;262:81-90.

12. Caspi A, Roberts B, Shiner R. Personality development: stability and change. Ann Rev Psychol. 2005;56:453-484.

13. McCrae RR, Costa Jr PT. Personality trait structure as a human universal. Am Psychol. 1997;52:509516.

14. Burcusa SL, lacono WG. Risk for recurrence in depression. Clin Psychol Rev. 2007;27:959-985.

15. Griffith JW, Zinbarg RE, Craske MG, Mineka S, Rose RD, Waters AM, et al. Neuroticism as a common dimension in the internalizing disorders. Psychol Med. 2010;40:1125-1136.

16. Kendler KS, Gatz M, Gardner CO, Pedersen NL. Personality and major depression: a Swedish longitudinal, population-based twin study. Arch Gen Psychiat. 2006;63:1113-1120.

17. Noteboom A, Beekman AT, Vogelzangs N, Penninx BW. Personality and social support as predictors of first and recurrent episodes of depression. J Affect Disord. 2016;190:156-161.

18. Hwang SI, Choi KI, Park OT, Park S-W, Choi ES, Yi S-H. Correlations between pre-morbid personality and depression scales in stroke patients. Ann Rehabil Med. 2011;35:328-336.

19. Storor DL, Byrne GJ. Pre-morbid personality and depression following stroke. Int Psychogeriatr. 2006;18:457-470.

20. Morris PL, Robinson RG. Personality neuroticism and depression after stroke. Int J Psychiatr Med. 1995;25:93-102.

21. Greenop KR, Almeida OP, Hankey GJ, van Bockxmeer F, Lautenschlager NT. Premorbid personality traits are associated with post-stroke behavioral and psychological symptoms: a three-month follow-up study in Perth, Western Australia. Int Psychogeriatr. 2009;21:1063-1071.

22. Brodaty H, Sachdev PS, Withall A, Altendorf A, Valenzuela MJ, Lorentz L. Frequency and clinical, neuropsychological and neuroimaging correlates of apathy following stroke--the Sydney Stroke Study. Psychol Med. 2005;35:1707-1716. 
23. Withall A, Brodaty H, Altendorf A, Sachdev PS. A longitudinal study examining the independence of apathy and depression after stroke: The Sydney Stroke Study. Int Psychogeriatr. 2011;23:264273.

24. Douven E, Schievink SH, Verhey FR, van Oostenbrugge RJ, Aalten P, Staals J, et al. The Cognition and Affect after Stroke-a Prospective Evaluation of Risks (CASPER) study: rationale and design. BMC Neurol. 2016;16:1-11.

25. Folstein MF, Folstein SE, McHugh PR. "Mini-mental state": a practical method for grading the cognitive state of patients for the clinician. J Pychiatr Res. 1975;12:189-198.

26. Jorm A. A short form of the Informant Questionnaire on Cognitive Decline in the Elderly (IOCODE): development and cross-validation. Psychol Med. 1994;24:145-153.

27. Costa PT, McCrae RR. The NEO personality inventory: Manual, form S and form R. Psychological Assessment Resources. 1985.

28. Hoekstra H, Ormel J, De Fruyt F. NEO personality questionnaires NEO-PI-R, NEO-FFI (in Dutch). Swet \& Zeitlinger BV, Lisse.1996.

29. Vollrath M, Torgersen S. Personality types and coping. Pers Indiv Differ. 2000;29:367-378.

30. Montgomery SA, Asberg M. A new depression scale designed to be sensitive to change. $\mathrm{Br} \mathrm{J}$ Psychiatry. 1979;134:382-389.

31. Kang H-J, Stewart R, Kim J-M, Jang J-E, Kim S-Y, Bae K-Y, et al. Comparative validity of depression assessment scales for screening poststroke depression. J Affect Disord. 2013;147:186-191.

32. Overbeek I, Schruers K, Griez E. Mini international neuropsychiatric interview: Nederlandse versie 5.0. 0. DSM-IV [Dutch version] Maastricht, The Netherlands: Universiteit Maastricht. 1999.

33. Marin RS, Biedrzycki RC, Firinciogullari S. Reliability and validity of the Apathy Evaluation Scale. Psychiatr Res. 1991;38:143-162.

34. Clarke DE, Ko JY, Kuhl EA, van Reekum R, Salvador R, Marin RS. Are the available apathy measures reliable and valid? A review of the psychometric evidence. J Psychosom Res. 2011;70:73-97.

35. Caeiro L, Ferro JM, Pinho e Melo T, Canhão P, Figueira ML. Post-stroke apathy: an exploratory longitudinal study. Cerebrovasc Dis. 2013;35:507-513.

36. Mahoney FI, Barthel DW. Barthel Index. Md State Med J. 1965;14:61-65.

37. Wongpakaran N, Wongpakaran T, van Reekum R. Social inhibition as a mediator of neuroticism and depression in the elderly. BMC Geriatr. 2012;12:1-7.

38. Yoon KL, Maltby J, Joormann J. A pathway from neuroticism to depression: examining the role of emotion regulation. Anxiety Stress Coping. 2013;26:558-572.

39. Cooper CL, Phillips LH, Johnston M, Whyte M, MacLeod MJ. The role of emotion regulation on social participation following stroke. Br J Clin Psychol. 2015;54:181-199.

40. Robert P, Onyike C, Leentjens A, Dujardin K, Aalten P, Starkstein S, et al. Proposed diagnostic criteria for apathy in Alzheimer's disease and other neuropsychiatric disorders. Eur Psychiatry. 2009;24:98-104.

41. Van Mierlo ML, Van Heugten CM, Post MW, De Kort PL, Visser-Meily JM. Psychological factors determine depressive symptomatology after stroke. Arch Phys Med Rehab. 2015;96:1064-1070.

42. Sollberger M, Stanley CM, Ketelle R, Beckman V, Growdon M, Jang J, et al. Neuropsychological correlates of dominance, warmth, and extraversion in neurodegenerative disease. Cortex. 2012;48:674-682.

43. Mendez Rubio M, Antonietti J, Donati A, Rossier J, Von Gunten A. Personality traits and behavioural and psychological symptoms in patients with mild cognitive impairment. Dement Geriatr Cogn. 2013;35:87-97.

44. Starkstein SE, Leentjens AF. The nosological position of apathy in clinical practice. J Neurol Neurosur Psychiatry. 2008;79:1088-1092.

45. Robinson RG, Kubos KL, Starr LB, Rao K, Price TR. Mood disorders in stroke patients. Importance of location of lesion. Brain. 1984;107:81-93. 
46. Gainotti G, Azzoni A, Marra C. Frequency, phenomenology and anatomical-clinical correlates of major post-stroke depression. Br J Psychiatry. 1999;175:163-167.

47. Towfighi A, Ovbiagele B, El Husseini N, Hackett ML, Jorge RE, Kissela BM, et al. Poststroke Depression: A Scientific Statement for Healthcare Professionals From the American Heart Association/American Stroke Association. Stroke. 2016;46:30-43.

48. Shi Y, Xiang Y, Yang Y, Zhang N, Wang S, Ungvari GS, et al. Depression after minor stroke: Prevalence and predictors. J Psychosom Res. 2015;79:143-147. 


\section{SUPPLEMENTAL DATA}

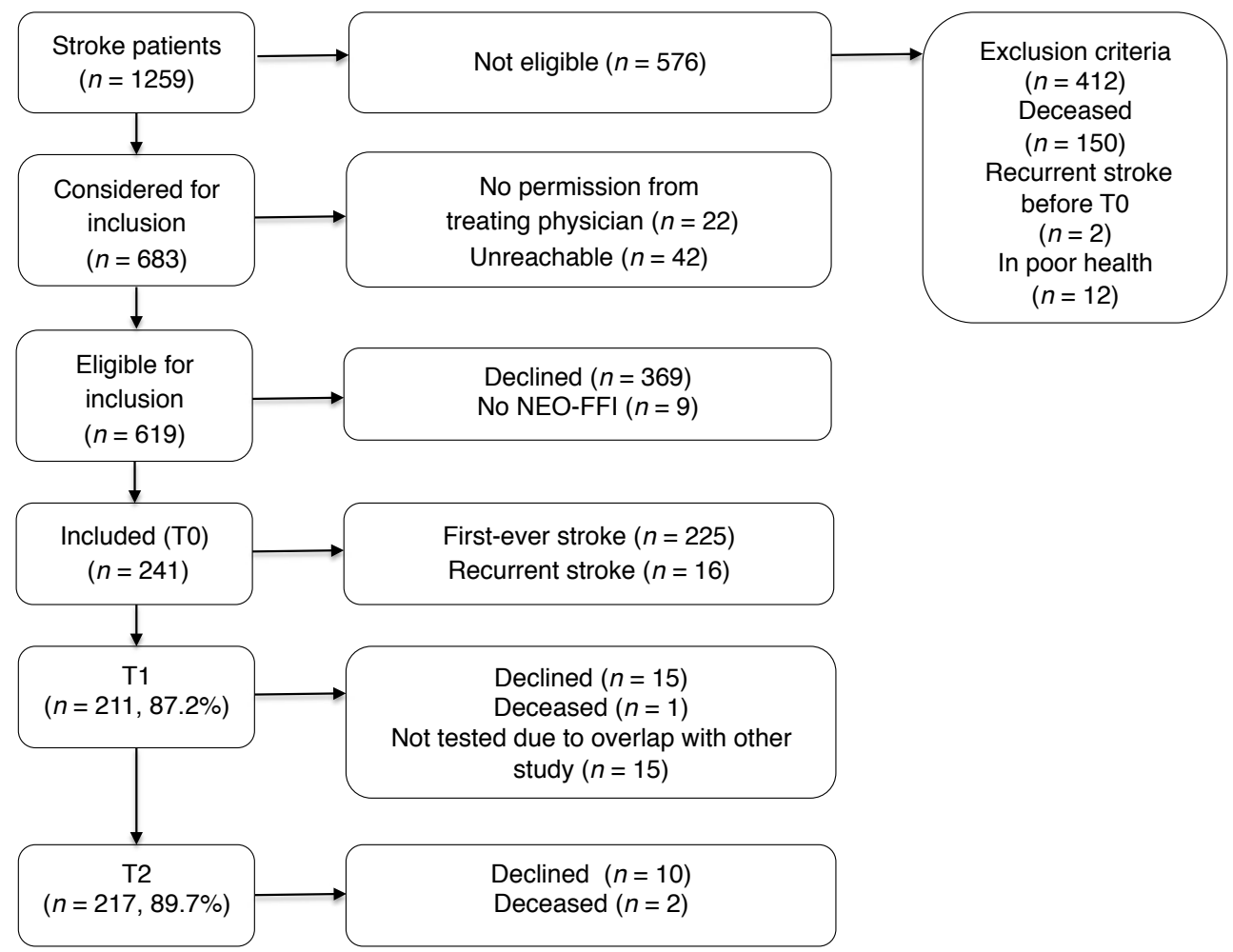

Supplementary Figure 1. Flowchart of inclusion process

Eighteen patients who did not participate at T1, agreed to participate at T2. T0 indicates baseline measurement 3 months post stroke; T1, follow-up 9 months post-stroke; T2, follow-up 15 months poststroke. 
Supplementary Table 1. Baseline characteristics for total sample, and separate for patients who completed all measurements and patients who dropped out at 1 or 2 time points

\begin{tabular}{|c|c|c|c|}
\hline Variable & $\begin{array}{l}\text { All patients T0 } \\
\quad(n=241)\end{array}$ & $\begin{array}{l}\text { Complete follow-up } \\
\qquad(n=219)\end{array}$ & $\begin{array}{l}\text { Dropouts } \\
(n=22)\end{array}$ \\
\hline Age in years (SD) & $67.31(11.78)$ & $66.99(11.56)$ & $70.47(13.71)$ \\
\hline Male sex, $n(\%)$ & $158(65.6)$ & $145(66.2)$ & $13(59.1)$ \\
\hline Low education, $n(\%)$ & $97(40.4)$ & $86(39.3)$ & $11(52.4)$ \\
\hline Middle education, $n(\%)$ & $84(35.0)$ & $78(35.6)$ & $6(28.6)$ \\
\hline High education, $n(\%)$ & $59(24.6)$ & $55(25.1)$ & $4(19.1)$ \\
\hline Time since stroke (months) & $2.93(0.45)$ & $2.92(0.43)$ & $3.07(0.61)$ \\
\hline First-ever stroke, $n(\%)$ & $225(93.4)$ & $204(93.2)$ & $21(95.5)$ \\
\hline Barthel score (SD) & $19.44(1.49)$ & $19.46(1.48)$ & $19.32(1.55)$ \\
\hline \multicolumn{4}{|l|}{ Personality score (SD) } \\
\hline Neuroticism & $31.60(4.89)$ & $31.63(4.94)$ & $31.27(4.50)$ \\
\hline Agreeableness & $36.54(4.60)$ & $36.56(4.55)$ & $36.36(5.13)$ \\
\hline Openness & $36.85(4.10)$ & $36.87(4.14)$ & $36.68(3.75)$ \\
\hline Extraversion & $38.25(4.19)$ & $38.29(4.23)$ & $37.82(3.82)$ \\
\hline Conscientiousness & $39.63(3.75)$ & $39.67(3.80)$ & $39.18(3.23)$ \\
\hline History of depression, $n(\%)$ & $55(22.8)$ & $49(22.4)$ & $6(27.3)$ \\
\hline Family history of depression, $n(\%)$ & $34(14.2)$ & $33(15.1)$ & $1(4.8)$ \\
\hline MADRS score TO (SD) & $6.06(5.84)$ & $6.26(5.92)$ & $4.18(4.73)$ \\
\hline AES-C score TO (SD) & $26.41(7.86)$ & $26.47(8.02)$ & $25.73(6.14)$ \\
\hline MINI minor / major depression, $n(\%)$ & $26(10.8)$ & $25(11.4)$ & $1(4.6)$ \\
\hline $\mathrm{MDD}, \mathrm{n}(\%)$ & $13(5.4)$ & $12(5.5)$ & $1(4.6)$ \\
\hline MIND, $n(\%)$ & $13(5.4)$ & 13. $(5.9)$ & $0(0.0)$ \\
\hline PSA, $n$ (\%) & $24(10.0)$ & $24(10.0)$ & $0(0.0)$ \\
\hline
\end{tabular}

Abbreviations: AES-C, Apathy Evaluation Scale Clinician-rated; MADRS, Montgomery-Åsberg Depression Rating Scale; MDD, major depressive disorder; MIND, minor depression; MINI, Mini International Neuropsychiatric Interview; PSA, post-stroke apathy; TO, baseline measurement 3 months post-stroke. ${ }^{\mathrm{a}} p<0.05,{ }^{\mathrm{b}} p<0.01$. 


\section{CHAPTER 6}




\section{IMAGING MARKERS ASSOCIATED WITH \\ THE DEVELOPMENT OF POST-STROKE \\ DEPRESSION AND APATHY}

Elles Douven

Julie Staals

Syenna HJ Schievink

Whitney M Freeze

Danique MJ Hellebrekers

Robin Wolz

Robert J van Oostenbrugge

Frans RJ Verhey

Sebastian Köhler

Pauline Aalten 


\section{ABSTRACT}

Background: It has been suggested that the development of post-stroke depression (PSD) and post-stroke apathy (PSA) may be more associated with generalized brain pathology, rather than with the stroke lesion itself. The present study aimed to investigate associations between imaging markers of lesion-related and generalized brain pathology and the development of PSD and PSA during a one-year follow-up.

Methods: In a prospective cohort study, 188 stroke patients received 3-Tesla MRI at baseline (three months post-stroke) for volumetric and visual rating of lesion-related, vascular, and degenerative brain pathology. Presence of lacunes, microbleeds, white matter hyperintensities, and enlarged perivascular spaces was summed to provide a measure of total cerebral small vessel disease (cSVD) burden. The Mini International Neuropsychiatric Interview and Apathy Evaluation Scale were administered at baseline and repeated at 6-and 12-month follow-up to define presence of PSD and PSA, respectively.

Results: Population-averaged logistic regression models showed that global brain atrophy $\left(\mathrm{OR}_{\mathrm{GEE}} 5.33,95 \% \mathrm{Cl} 1.99-14.25\right)$ and severe burden of CSVD $\left(\mathrm{OR}_{\mathrm{GEE}} 3.04,95 \% \mathrm{Cl} 1.20-7.69\right)$ were significantly associated with the odds of developing PSA, independent of stroke lesion volume and co-morbid PSD. Medium cSVD burden $\left(\mathrm{OR}_{\mathrm{GEE}} 2.92,95 \% \mathrm{Cl} 1.09-7.78\right)$ was significantly associated with the odds of developing PSD, independent of stroke lesion volume, co-morbid PSA, and pre-stroke history of depression. No associations were found with lesion-related imaging markers.

Conclusions: The results suggest that generalized degenerative and vascular brain pathology, rather than lesion-related pathology, is an important predictor for the development of PSA, and less strongly for PSD. 


\section{INTRODUCTION}

Post-stroke depression (PSD) and post-stroke apathy (PSA) are frequent neuropsychiatric consequences of stroke, with estimated mean prevalences around 30\% based on previous meta-analyses. ${ }^{1,2}$ Both PSD and PSA are known to be associated with poor clinical outcome., ${ }^{2,3}$ Though there is substantial overlap in symptoms ${ }^{4-6}$ these syndromes seem to develop from different anatomical bases. Earlier studies suggested that PSD is associated with left anterior lesions, ${ }^{7,8}$ and PSA with right hemispheric subcortical lesions. ${ }^{9,10}$ However, meta-analytic studies did not show strong support for differential lesion locations associated with PSD and PSA. ${ }^{11-13}$ Recently, studies have focused on damage to neuronal networks being involved in the development of PSD and PSA, ${ }^{14-16}$ with evidence for different subcircuits associated with PSD ${ }^{17}$ and PSA. ${ }^{18}$ As damage to these networks can arise from both stroke lesion-related and more generalized brain damage, a broad range of imaging markers may be involved in the development of PSD and PSA.

Apathy and depression are prevalent in patients with cerebral small vessel disease (CSVD), ${ }^{19-24}$ a condition that is radiologically characterized by extensive white matter hyperintensities (WMH), lacunes, enlarged perivascular spaces, and cerebral microbleeds (CMBs). In addition to the contribution of individual cSVD markers, the role of cumulative or total cSVD burden may be of interest, as a recent crosssectional study showed that increased total burden of CSVD markers on magnetic resonance imaging (MRI) was associated with PSD in lacunar stroke patients three months post-stroke. ${ }^{23}$ Next to cSVD imaging markers, previous studies have shown that atrophy might play a role in the development of PSD and PSA. PSD has been associated with visually rated (sub)cortical atrophy ${ }^{25}$ and frontal lobe atrophy. ${ }^{26}$ PSA has been associated with visually rated frontal cortical atrophy ${ }^{27}$ and with volume reductions in the posterior cingulate cortex over the first six months poststroke. ${ }^{28}$ Studies using a volumetric measure of brain atrophy to study associations with PSD or PSA ${ }^{28}$ are relatively scarce and the role of (generalized and focal) brain atrophy in the development of these neuropsychiatric syndromes remains therefore poorly understood.

More insight in the underlying pathophysiology of PSD and PSA is important, as it may help to design better treatment strategies and prevention of these syndromes. In the present study, we aimed to investigate the association between stroke-related imaging markers and more generalized degenerative and vascular brain pathology and the development of PSD and PSA during a one-year followup period. 


\section{METHODS}

\section{PARTICIPANTS AND PROCEDURE}

This is a substudy of the Cognition and Affect after Stroke, a Prospective Evaluation of Risks (CASPER) study, a prospective clinical stroke cohort study that aims to examine predictors of cognitive impairment, apathy, and depression. The study was approved by the Medical Ethics Committee of Maastricht University Medical Center (MUMC+). Patients admitted to the Stroke Unit or out-patient clinic of MUMC+ or Zuyderland Hospital, the Netherlands, for a non-fatal ischemic or hemorrhagic stroke were approached for participation between June 2013 and November 2015. Patients were included after informed consent. Details of the study protocol have been published elsewhere. ${ }^{29}$

At baseline, neuropsychiatric questionnaires were administered by a trained research psychologist and socio-demographic information was recorded. A subset $(n=189$ ) received 3 -Tesla (3T) structural brain MRI. To avoid interference with acute care and rehabilitation, these baseline measurements were scheduled approximately three months after stroke onset (TO). At 6-month (T1) and 12-month (T2) follow-up, neuropsychiatric assessments were repeated.

\section{CLINICAL MEASURES}

Patients were classified as having PSD if they fulfilled criteria for Major Depressive Disorder (MDD) or minor depression (MIND) on the Mini International Neuropsychiatric Interview ${ }^{30}$ on at least one assessment during the one-year follow-up period. PSA was defined as a score $\geq 37^{6}$ on either the clinician-rated Apathy Evaluation Scale (AES-C) or informant-rated AES (AES-I) ${ }^{31}$ on at least one assessment during the one-year follow-up period. The Barthel Index was used as a global measure of impairment in activities of daily living. ${ }^{32}$

\section{MAGNETIC RESONANCE IMAGING}

Patients who were capable underwent a 3T structural brain MRI at T0 (Philips Achieva, Philips Medical Systems, Best, the Netherlands). The MR protocol consisted of axial T1-weighted, T2-weighted, fluid-attenuation inversion recovery (FLAIR), diffusion weighted, and susceptibility weighted sequences (for details see Douven et al. $\left.{ }^{29}\right)$.

Volumetric imaging markers. Freesurfer software ${ }^{33}$ was used to segment cortical gray and white matter on T1-weighted scans. WMH volume was segmented automatically on FLAIR images according to an automatic brain tissue segmentation method for $\mathrm{WMH}$ segmentation ${ }^{34}$ and a T1-weighted sequence was used for anatomical 
reference. A manual correction was performed by two trained researchers (ED and $D H$ ), under supervision of an experienced neurologist (JS) (Supplementary Figure 1A). WMH volume was normalized to the intracranial volume (ICV) and a natural log transformation was applied to normalize the data distribution.

Symptomatic and old stroke lesions and lacunes were indicated by an experienced neurologist (JS) and segmented manually (Supplementary Figure 1B). Stroke lesion volume was normalized to ICV and divided into tertiles (i.e. small, medium, and large lesion volumes). Stroke patients without a visible symptomatic lesion on MRI were classified into the small stroke volume group. The ratio of brain parenchymal volume to total ICV was used to measure whole brain volume. Global brain atrophy (GA) was calculated as 1 minus brain volume, and multiplied by 10 to ease the interpretation of odds ratios. Hippocampal volume (HCV) was obtained using the Learning Embeddings for Atlas Propagation (LEAP) method of Wolz et al. ${ }^{35} \mathrm{HCV}$ was normalized by dividing the native space volume by the affine scaling factor, which was derived from LEAP as well. ${ }^{36}$ Scores were divided by one thousand to ease interpretation of odds ratios.

Visual imaging markers. Stroke location, laterality, and lesion type (i.e. cortical/ non-lacunar, lacunar, infratentorial), presence of lacunes and other old infarcts, presence of $\mathrm{CMBs}$, medial temporal lobe atrophy (MTA), ${ }^{37}$ presence of extensive WMH according to the Fazekas scale, ${ }^{38}$ and enlarged perivascular spaces in basal ganglia were visually rated by an experienced neurologist (JS) (for details see Data Supplement 1).

A well-validated scale for total cSVD burden on MRI was used, which is based on counting the presence of lacunes, CMBs, perivascular spaces and extensive WMH. ${ }^{39}$ It should be noted that, as in contrast with the originally developed ordinal CSVD scale, we used susceptibility weighted imaging (SWI) for counting CMBs. CSVD burden scores were recoded into three categories representing low (score $0-1$ ), medium (score 2), and severe (score 3-4) burden.

\section{STATISTICAL ANALYSES}

All statistical analyses were performed with Stata version 13.1 (StataCorp, TX, USA). Statistical significance was set at 0.05 (two-sided tests). Baseline differences between groups were tested using $\mathrm{x}^{2}$ tests and $t$-tests, as appropriate. Associations with imaging markers were corrected for age, sex, and highest level of education in logistic regression models.

A generalized estimating equation (GEE) for the binomial family with a logit-link function (population-averaged logistic model) was used to evaluate the effect of individual baseline imaging markers on the odds of PSD and PSA at any time point. 
This yielded marginal odds ratios $\left(\mathrm{OR}_{\mathrm{GEE}}\right)$ and $95 \%$ confidence intervals $(95 \% \mathrm{Cl})$. Each imaging marker was tested separately in model 1, which was corrected for age, sex, highest level of education (low, middle, high), and time (T0, T1, T2). The latter was included to increase model efficiency by making maximum use of all available data and averaging effects over all three time points. For the tests with PSD as outcome, history of depression was additionally added to model 1 in a second step. In the next model, individual imaging predictors with a $p$-value $<.10$ in the previous model were added to the multivariable model, which was corrected for stroke volume in addition to the previous covariates. Lastly, the model was repeated with variables with a $p$-value $<.10$, and models with PSD as outcome were additionally corrected for PSA, and vice versa, to control for overlap in symptomatology.

\section{RESULTS}

Of the 250 included stroke patients, brain 3T MRI at baseline was available for 189 patients. One patient was excluded from the analyses because responses given on questionnaires were considered unreliable, resulting in a total of 188 patients. Of them, only 168 patients had volumetric imaging data available (17 patients were scanned for clinical purposes according to a different scanning protocol; in three patients there were technical problems during image processing). CMBs were counted on SWI, which were only available for 147 stroke patients. Patients excluded for the present study $(n=62)$ were significantly older, more frequently female, more likely to be low educated, and had a lower Barthel Index score compared with patients who were included in the present study $(n=188)$ (Supplementary Table 1). No difference was found in frequency of PSA or PSD between the groups at T0. Of the 188 patients at baseline, 165 (87.8\%) patients completed T1 and 172 (91.5\%) patients completed T2 (Supplementary Figure 2). No difference was found in frequency of PSA or PSD at T0 between patients lost to follow-up at one or two time points $(n=14)$ and patients who completed all measurements $(n=174)$ (Supplementary Table 2).

Table 1 shows the baseline characteristics of the total sample, and also separate by PSD and PSA status at baseline. Compared with the no-PSA group, patients with PSA had a higher WMH volume, more GA, and a higher frequency of severe cSVD burden. However, these associations were not significant anymore after correction for age, sex, and highest level of education. No differences were found between patients with and without PSD at TO. 
Table 1. Patient characteristics of the total sample and by PSD and PSA status at baseline

\begin{tabular}{|c|c|c|c|c|c|}
\hline Variables & $\begin{array}{l}\text { Total sample } \\
\quad(n=188)\end{array}$ & $\begin{array}{l}\text { No PSD } \\
(n=168)\end{array}$ & $\begin{array}{c}\text { PSD } \\
(n=20)\end{array}$ & $\begin{array}{l}\text { No PSA } \\
(n=157)\end{array}$ & $\begin{array}{c}\text { PSA } \\
(n=31)\end{array}$ \\
\hline Age in years, mean (SD) & $65.4(11.1)$ & $65.5(10.9)$ & $64.7(12.8)$ & $64.2(11.0)$ & $71.5(9.7)^{b}$ \\
\hline Male sex, $n(\%)$ & $133(70.7)$ & $123(73.2)$ & $10(50.0)^{\mathrm{b}}$ & $109(69.4)$ & $24(77.4)$ \\
\hline \multicolumn{6}{|l|}{ Education, $n(\%)$} \\
\hline Low education & $68(36.4)$ & $61(36.5)$ & $7(35.0)$ & $54(34.6)$ & $14(45.2)$ \\
\hline Middle education & $70(37.4)$ & $59(35.3)$ & $11(55.0)$ & $57(36.5)$ & $13(41.9)$ \\
\hline High education & $49(26.2)$ & $47(28.1)$ & $2(10.0)$ & $45(28.9)$ & $4(12.9)$ \\
\hline Barthel Index, mean (SD) & $19.7(0.9)$ & $19.7(0.9)$ & $19.7(0.8)$ & $19.7(0.9)$ & $19.7(0.8)$ \\
\hline History of depression, $n(\%)$ & $40(21.3)$ & $30(17.9)$ & $10(50.0)^{\mathrm{b}}$ & $32(20.4)$ & $8(25.8)$ \\
\hline \multicolumn{6}{|l|}{ Lesion characteristics* } \\
\hline Ischemic stroke, $n(\%)$ & $176(93.6)$ & $157(93.5)$ & $19(95.0)$ & $147(93.6)$ & $29(93.6)$ \\
\hline Left-sided lesion $(n=177), n(\%)$ & $80(45.2)$ & $74(47.1)$ & $6(30.0)$ & $66(44.6)$ & $14(48.3)$ \\
\hline Frontal lesion $(n=122), n(\%)$ & $41(33.6)$ & $39(35.8)$ & $2(15.4)$ & $35(34.3)$ & $6(30.0)$ \\
\hline Basal ganglia lesion $(n=138), n(\%)$ & $7(5.1)$ & $5(4.1)$ & $2(13.3)$ & $5(4.3)$ & $2(9.1)$ \\
\hline \multicolumn{6}{|l|}{ Stroke lesion volume $(n=168), n(\%)$} \\
\hline Small & $56(33.3)$ & $50(33.6)$ & $6(31.6)$ & $47(33.3)$ & $9(33.3)$ \\
\hline Medium & $56(33.3)$ & $52(34.9)$ & $4(21.1)$ & $50(35.5)$ & $6(22.2)$ \\
\hline Large & $56(33.3)$ & $47(31.5)$ & $9(47.4)$ & $44(31.2)$ & $12(44.4)$ \\
\hline \multicolumn{6}{|l|}{ Stroke type, $(n=174), n(\%)$} \\
\hline Cortical/non-lacunar & $99(56.9)$ & $87(56.1)$ & $12(63.2)$ & $81(55.9)$ & $18(62.1)$ \\
\hline Lacunar & $54(31.0)$ & $49(31.6)$ & $5(26.3)$ & $46(31.7)$ & $8(27.6)$ \\
\hline Infratentorial & $21(12.1)$ & $19(12.3)$ & $2(10.5)$ & $18(12.4)$ & $3(10.3)$ \\
\hline \multicolumn{6}{|l|}{ Background damage on MRI* } \\
\hline Lacunes and other old infarcts, $n(\%)$ & $75(39.9)$ & $66(39.3)$ & $9(45.0)$ & $59(37.6)$ & $16(51.6)$ \\
\hline Cerebral microbleeds, $n(\%)(n=147)$ & $86(58.5)$ & $77(59.7)$ & $9(50.0)$ & $70(56.5)$ & $16(69.6)$ \\
\hline ePVS in basal ganglia, $n(\%) n=187$ ) & $105(56.2)$ & $94(56.3)$ & $11(55.0)$ & $85(54.5)$ & $20(64.5)$ \\
\hline Abnormal MTA left, $n(\%)(n=170)$ & $27(15.9)$ & $25(16.6)$ & $2(10.5)$ & $22(15.5)$ & $5(17.9)$ \\
\hline Abnormal MTA right, $n(\%)(n=170)$ & $36(21.2)$ & $32(21.2)$ & $4(21.1)$ & $29(20.4)$ & $7(25.0)$ \\
\hline WMH volume $(\%$ ICV $)(n=168)$ & $0.65(0.97)$ & $0.64(0.95)$ & $0.74(1.11)$ & $0.56(0.91)$ & $1.09(1.15)$ \\
\hline $\mathrm{GA}(\% \mathrm{ICV})(n=168)$ & $0.33(0.04)$ & $0.33(0.04)$ & $0.34(0.04)$ & $0.33(0.04)$ & $0.35(0.04)^{a}$ \\
\hline HCV left $\left(\mathrm{mm}^{3}\right)$, mean $(\mathrm{SD})(n=168)$ & $\begin{array}{l}2752.7 \\
(325.8)\end{array}$ & $\begin{array}{l}2746.2 \\
(328.2)\end{array}$ & $\begin{array}{l}2803.9 \\
(310.0)\end{array}$ & $\begin{array}{l}2763.7 \\
(338.6)\end{array}$ & $\begin{array}{l}2695.2 \\
(245.3)\end{array}$ \\
\hline $\mathrm{HCV}$ right $\left(\mathrm{mm}^{3}\right)$, mean $(\mathrm{SD})(n=168)$ & $\begin{array}{l}2809.6 \\
(366.6)\end{array}$ & $\begin{array}{l}2811.9 \\
(359.7)\end{array}$ & $\begin{array}{l}2791.7 \\
(426.9)\end{array}$ & $\begin{array}{l}2840.8 \\
(371.0)\end{array}$ & $\begin{array}{c}2647.1 \\
(298.4)^{a}\end{array}$ \\
\hline \multicolumn{6}{|l|}{ cSVD burden category $(n=147)$} \\
\hline $0-1$ & $72(49.0)$ & $65(50.4)$ & $7(38.9)$ & $65(52.4)$ & $7(30.4)$ \\
\hline 2 & $40(27.2)$ & $35(27.1)$ & $5(27.8)$ & $35(28.2)$ & $5(21.7)$ \\
\hline $3-4$ & $35(23.8)$ & $29(22.5)$ & $6(33.3)$ & $24(19.4)$ & $11(47.8)^{a}$ \\
\hline
\end{tabular}

*Imaging markers were corrected for age, sex, and highest level of education.

Note: Differences were tested between no PSD and PSD, as well as between no PSA and PSA.

${ }^{\mathrm{a}} \mathrm{p}<.10,{ }^{\mathrm{b}} \mathrm{p}<.05$

Abbreviations: cSVD, cortical small vessel disease; ePVS, enlarged perivascular spaces; GA, global brain atrophy; HCV indicates hippocampal volume; PSA, post-stroke apathy; PSD, post- stroke depression; MTA, medial temporal lobe atrophy; WMH, white matter hyperintensities.

\section{IMAGING MARKERS ASSOCIATED WITH PSD IN THE FIRST YEAR AFTER STROKE}

PSD was present in 20 patients $(10.6 \%)$ at T0, 22 patients $(13.3 \%)$ at T1, and 22 patients (12.8\%) at T2. Table 2 provides an overview of the results of the models with PSD as outcome. There was a significant association between GA and the marginal odds of developing PSD during the one-year follow-up period after adjusting for 
age, sex, and highest level of education $\left(\mathrm{OR}_{\mathrm{GEE}} 2.82,95 \% \mathrm{Cl} 1.07-7.44\right)$. As there was a significant difference in frequency of history of depression between patients with and without PSD, we tested whether history of depression was a significant covariate. History of depression was significantly associated with the marginal odds of developing PSD $\left(\mathrm{OR}_{\mathrm{GEE}} 4.14,95 \% \mathrm{Cl}\right.$ 1.98-8.66), and therefore we corrected for this in the second step. The results showed that the association with GA was no longer significant after correction $\left(\mathrm{OR}_{\mathrm{GEE}} 2.20,95 \% \mathrm{Cl}\right.$ 0.80-6.07). Medium, but not severe, total cSVD burden was significantly associated with the marginal odds of developing PSD during the one-year follow-up $\left(\mathrm{OR}_{\mathrm{GEE}} 2.42,95 \% \mathrm{Cl} 1.01-5.80\right)$, also after additional correction for history of depression $\left(\mathrm{OR}_{\mathrm{GEE}} 3.11,95 \% \mathrm{Cl} 1.24\right.$ 7.79). In the multivariable model, which was also corrected for stroke lesion volume, the association for medium total cSVD $\left(\mathrm{OR}_{\mathrm{GEE}} 3.31,95 \% \mathrm{Cl} 1.16-9.46\right)$, but not GA $\left(\mathrm{OR}_{\mathrm{GEE}} 2.20,95 \% \mathrm{Cl}\right.$ 0.80-6.07), remained significantly associated with PSA. After we controlled in a final step for comorbid PSA, medium total cSVD burden remained significantly associated with the odds of developing PSD $\left(\mathrm{OR}_{\mathrm{GEE}} 2.92,95 \% \mathrm{Cl} 1.09\right.$ 7.78). The inclusion of cognitive impairment and presence of old infarcts as covariates (Supplementary Table 3) or the exclusion of hemorrhagic strokes (Supplementary Table 4) in a sensitivity analysis did not substantively change the results.

\section{IMAGING MARKERS ASSOCIATED WITH PSA IN THE FIRST YEAR AFTER STROKE}

PSA was present in 31 patients (16.5\%) at T0, 40 patients $(24.2 \%)$ at T1, and 37 patients $(21.5 \%)$ at T2. Table 3 provides an overview of the results of the marginal logistic models with PSA as outcome. There was a significant association between GA and the odds of developing PSA during the one-year follow-up period after adjusting for age, sex, and highest level of education $\left(\mathrm{OR}_{\mathrm{GEE}} 5.92,95 \% \mathrm{Cl} 2.36\right.$ 14.86). In addition, severe CSVD burden was significantly associated with the odds of developing PSA during the one-year follow-up period $\left(\mathrm{OR}_{\mathrm{GEE}} 2.92,95 \% \mathrm{Cl} 1.28\right.$ 6.67). In the multivariable model, which was also corrected for stroke lesion volume, the association for $\mathrm{GA}\left(\mathrm{OR}_{\mathrm{GEE}} 6.51,95 \% \mathrm{Cl} 2.54-16.73\right)$, and severe cSVD burden $\left(\mathrm{OR}_{\mathrm{GEE}} 3.36,95 \% \mathrm{Cl}\right.$ 1.05-10.72), were significantly associated with PSA. After we controlled in a final step for comorbid PSD, both GA (OR ${ }_{\mathrm{GEE}} 5.33,95 \% \mathrm{Cl}$ 1.99-14.25) and severe cSVD burden $\left(\mathrm{OR}_{\mathrm{GEE}} 3.61,95 \% \mathrm{Cl} 1.34-9.68\right)$ remained significantly associated with the odds of developing PSA. The inclusion of cognitive impairment and presence of old infarcts as covariates (Supplementary Table 3 ) in a sensitivity analysis did not substantively change the results. When hemorrhagic strokes $(n=12)$ were excluded from the analysis the same results were found, but in addition also large stroke lesion volume was significantly associated with the odds of developing PSA (OR ${ }_{\mathrm{GEE}} 2.48,95 \% \mathrm{Cl} 1.05-5.88$ ) (Supplementary Table 4). 


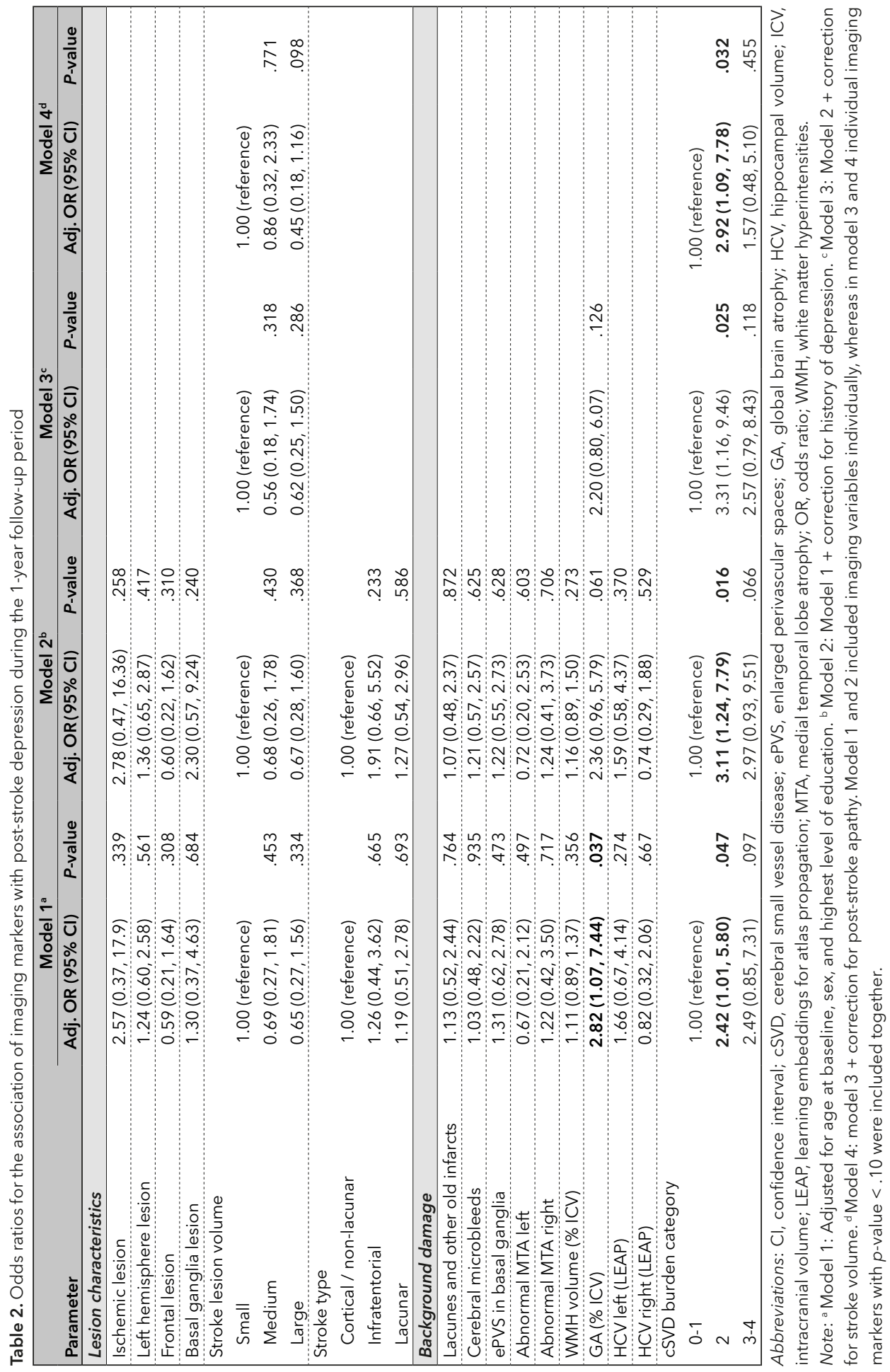




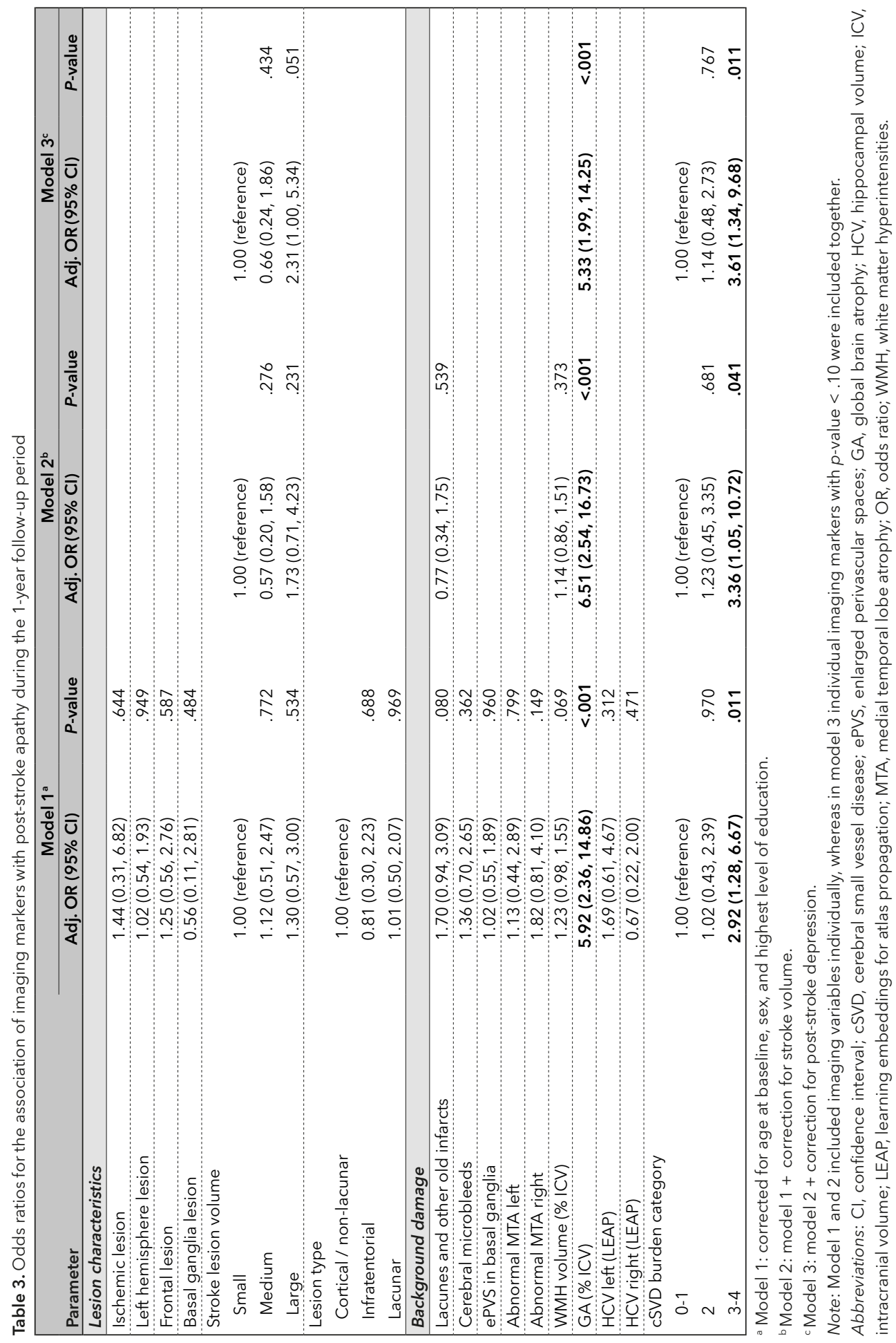




\section{DISCUSSION}

The present study aimed to investigate the association between stroke lesionrelated, general vascular, and neurodegenerative imaging markers assessed at three months post-stroke and the development of PSD and PSA during a oneyear follow-up. In adjusted models, patients with more GA or higher total burden of cSVD at baseline had elevated odds for the presence of PSA, independent of stroke lesion volume and co-morbid PSD. Patients with more GA or medium cSVD burden showed elevated odds for the presence of PSD after adjusting for relevant confounders. Further analyses suggested that the association of PSD with global brain atrophy was however due to co-morbid PSA, as only medium cSVD burden remained significantly related to PSD after correction for co-morbid PSA and stroke lesion volume. No associations were found between PSD/PSA and lesionrelated imaging markers (i.e. location, type, or stroke lesion volume).

Our findings suggest that GA and total cSVD burden are important predictors of PSA. These findings were independent from co-morbid PSD and not mediated by cognitive impairment or history of stroke, as the inclusion of these covariates in the analysis did not change the results substantively. Our findings are supported by different studies in stroke as well as general population studies. In two longitudinal stroke studies with 6-month follow-up, frontal cortical atrophy ${ }^{27}$ and atrophic change in the dorsal posterior cingulate $\operatorname{corte}^{28}$ were associated with PSA. However, these studies used a visual rating scale for atrophy ${ }^{27}$ or had a small sample size. ${ }^{28}$ In patients with symptomatic atherosclerotic disease, an association was found between greater cortical volume loss and anhedonia. ${ }^{40}$ This is of interest, as anhedonia is a core symptom of depression, ${ }^{41}$ which overlaps with the symptomatology of apathy. In a large population-based study of healthy older adults without dementia, apathy symptoms were associated with a reduction of gray and white matter volume. ${ }^{42}$ Noteworthy, these associations were found to be independent of depression. Previous studies have indicated that GA is not necessarily of degenerative origin, but is often found to be associated with CSVD. ${ }^{43}$ However, in contrast with the other CSVD markers included in the total CSVD burden score, atrophy is not specifically due to CSVD, as it may also occur in other conditions and is part of normal aging..$^{39}$ Therefore, GA was not considered as a marker of CSVD burden here.

Severe cSVD burden was associated with PSA, while medium cSVD burden was associated with PSD. A possible explanation for these findings could be that particularly in patients with severe CSVD burden PSD overlaps largely with PSA, as without correction for co-morbid PSA and stroke volume the association between PSD and severe CSVD almost reached significance. A previous cross-sectional study 
found that greater total cSVD burden was associated with the presence of PSD at three months after acute lacunar stroke. ${ }^{23}$ Though associations between individual MRI markers of cSVD and apathy have been found before, ${ }^{19,22,44}$ this is the first study that found an important role of total cSVD burden in the development of PSA, suggesting that a global or cumulative brain burden is more important in the development of these neuropsychiatric syndromes than the presence or location of single symptomatic lesions in stroke patients. Interestingly, these findings are in line with the suggestion that cumulative brain pathology, rather than lesion location or size, plays an important role in the development of PSA and PSD, ${ }^{14-16}$ by damaging microstructural networks that are involved in the regulation of mood and motivational processes.

The present study has several important strengths. First, a broad range of imaging markers were assessed in relation to PSD and PSA, including both lesion-related markers and markers of background vascular and degenerative pathology, by using both visual and volumetric ratings. Other strengths are the longitudinal design and the minimal loss to follow-up. Presence of PSD and PSA was determined by validated measurement instruments and assessed at several timepoints, enabling us to evaluate predictive associations. However, some limitations should be addressed. In a quarter of patients, no visible symptomatic stroke lesion was present on MRI. This limited the possibility to look at associations with specific lesion locations. In addition, by including the time variable in the marginal logistic GEE models, the models are based on the assumption that associations remain stable over time, irrespective of whether PSD or PSA was measured the first, second, or third measurement. Therefore, we can only conclude that we found associations with the development of PSD or PSA over the one-year follow-up period, but cannot tell whether these associations were stronger for a certain time point. This could have been studied by looking at the interaction with time, but the small incidences of PSD and PSA in our sample resulted in lack of power for these analyses. Also, the exclusion of patients with severe aphasia and other neurological or psychiatric conditions makes the sample less representative for a general stroke population.

In conclusion, GA and severe CSVD burden, rather than lesion-related markers, were associated with the development of PSA, independent of PSD. Only medium CSVD burden was associated with the development of PSD, independent of PSA and pre-stroke history of depression. Large stroke cohort studies with long-term follow-up and repeated neuroimaging over time are needed to establish how generalized degenerative and vascular brain pathology, and changes therein, are involved in the development of PSD and PSA. 


\section{REFERENCES}

1. Hackett ML, Yapa C, Parag V, Anderson CS. Frequency of depression after stroke a systematic review of observational studies. Stroke. 2005;36:1330-1340.

2. van Dalen JW, van Charante EPM, Nederkoorn PJ, van Gool WA, Richard E. Poststroke apathy. Stroke. 2013;44:851-860.

3. Ayerbe L, Ayis S, Wolfe CD, Rudd AG. Natural history, predictors and outcomes of depression after stroke: Systematic review and meta-analysis. Br J Psychiatry. 2013;202:14-21.

4. Starkstein SE, Ingram L, Garau M, Mizrahi R. On the overlap between apathy and depression in dementia. J Neurosurg Psychiatry. 2005;76:1070-1074.

5. Levy ML, Cummings JL, Fairbanks LA, Masterman D, Miller BL, Craig AH, et al. Apathy is not depression. J Neuropsychiatry Clin Neurosci. 1998;10:314-319.

6. Withall A, Brodaty H, Altendorf A, Sachdev PS. A longitudinal study examining the independence of apathy and depression after stroke: The sydney stroke study. Int Psychogeriatr. 2011;23:264273.

7. Robinson RG, Kubos KL, Starr LB, Rao K, Price TR. Mood disorders in stroke patients. Importance of location of lesion. Brain. 1984;107:81-93.

8. Hama S, Yamashita H, Shigenobu M, Watanabe A, Kurisu K, Yamawaki S, et al. Post-stroke affective or apathetic depression and lesion location: Left frontal lobe and bilateral basal ganglia. Eur Arch Psychiatry Clin Neurosci. 2007;257:149-152.

9. Starkstein SE, Fedoroff JP, Price TR, Leiguarda R, Robinson RG. Apathy following cerebrovascular lesions. Stroke. 1993;24:1625-1630.

10. Caeiro L, Ferro JM, Figueira ML. Apathy in acute stroke patients. Eur J Neurol. 2012;19:291-297.

11. Carson AJ, MacHale S, Allen K, Lawrie SM, Dennis M, House A, et al. Depression after stroke and lesion location: A systematic review. Lancet. 2000;356:122-126.

12. Wei N, Yong W, Li X, Zhou Y, Deng M, Zhu H, et al. Post-stroke depression and lesion location: A systematic review. J Neurology. 2015;262:81-90.

13. Caeiro L, Ferro JM, Costa J. Apathy secondary to stroke: A systematic review and meta-analysis. Cerebrovasc Dis. 2013;35:23-39.

14. Tang WK, Lu JY, Chen YK, Chu WCW, Mok V, Ungvari GS, et al. Association of frontal subcortical circuits infarcts in poststroke depression: A magnetic resonance imaging study of 591 chinese patients with ischemic stroke. J Geriatr Psychiatry Neurol. 2011;24:44-49.

15. Terroni L, Amaro E, losifescu DV, Tinone G, Sato JR, Leite CC, et al. Stroke lesion in cortical neural circuits and post-stroke incidence of major depressive episode: A 4-month prospective study. World J Biol Psychiatry. 2011;12:539-548.

16. Vataja R, Pohjasvaara T, Leppavuori A, Mantyla R, Aronen HJ, Salonen O, et al. Magnetic resonance imaging correlates of depression after ischemic stroke. Arch Gen Psychiatry. 2001;58:925-931.

17. Yang S, Hua P, Shang X, Cui Z, Zhong S, Gong G, et al. A significant risk factor for poststroke depression: The depression-related subnetwork. J Psychiatry Neurosci. 2015;40:259-268.

18. Yang S, Hua P, Shang X, Cui Z, Zhong S, Gong G, et al. Deficiency of brain structural sub-network underlying post-ischaemic stroke apathy. Eur J Neurol. 2015;22:341-347.

19. Lavretsky $\mathrm{H}$, Zheng $\mathrm{L}$, Weiner MW, Mungas $\mathrm{D}$, Reed B, Kramer JH, et al. The mri brain correlates of depressed mood, anhedonia, apathy, and anergia in older adults with and without cognitive impairment or dementia. Int J Geriatr Psychiatry. 2008;23:1040-1050.

20. O'brien JT, Firbank MJ, Krishnan MS, van Straaten EC, van der Flier WM, Petrovic K, et al. White matter hyperintensities rather than lacunar infarcts are associated with depressive symptoms in older people: The ladis study. Am J Geriatr Psychiatry. 2006;14:834-841.

21. Pantoni L. Cerebral small vessel disease: From pathogenesis and clinical characteristics to therapeutic challenges. Lancet Neurol. 2010;9:689-701. 
22. Reyes S, Viswanathan A, Godin O, Dufouil C, Benisty S, Hernandez K, et al. Apathy a major symptom in cadasil. Neurology. 2009;72:905-910.

23. Zhang $X$, Tang $Y$, Xie $Y$, Ding $C$, Xiao J, Jiang $X$, et al. Total magnetic resonance imaging burden of cerebral small-vessel disease is associated with post-stroke depression in patients with acute lacunar stroke. Eur J Neurol. 2017;24:374-380.

24. Pavlovic AM, Pekmezovic T, Zidverc Trajkovic J, Svabic Medjedovic T, Veselinovic N, Radojicic A, et al. Baseline characteristic of patients presenting with lacunar stroke and cerebral small vessel disease may predict future development of depression. Int J Geriatr Psychiatry. 2016;31:58-65.

25. Astrom M, Adolfsson R, Asplund K. Major depression in stroke patients. A 3-year longitudinal study. Stroke. 1993;24:976-982.

26. Tang WK, Chen YK, Lu JY, Mok VCT, Chu WCW, Ungvari GS, et al. Frontal lobe atrophy in depression after stroke. Stroke Res Treatment. 2013;2013:424769-424772.

27. Mihalov J, Mikula P, Budiš J, Valkovič P. Frontal cortical atrophy as a predictor of poststroke apathy. J Geriatr Psychiatry Neurolog. 2016:29;171-176.

28. Matsuoka K, Yasuno F, Taguchi A, Yamamoto A, Kajimoto K, Kazui H, et al. Delayed atrophy in posterior cingulate cortex and apathy after stroke. Int J Geriatr Psychiatry. 2015;30:566-572.

29. Douven E, Schievink SH, Verhey FR, van Oostenbrugge RJ, Aalten P, Staals J, et al. The cognition and affect after stroke-a prospective evaluation of risks (casper) study: Rationale and design. BMC Neurol. 2016;16:1-11.

30. Overbeek T, Schruers K, Griez E. Mini: Mini international neuropsychiatric interview, Dutch version 5.0. 0 (DSM-IV). Maastricht: University of Maastricht. 1999

31. Marin RS, Biedrzycki RC, Firinciogullari S. Reliability and validity of the apathy evaluation scale. Psychiatry Res. 1991;38:143-162

32. Mahoney Fl, Barthel DW. Functional evaluation: The Barthel index. Md State Med J. 1965;14:6165.

33. Fischl B. Freesurfer. Neuroimage. 2012;62:774-781.

34. De Boer R, Vrooman HA, Van Der Lijn F, Vernooij MW, Ikram MA, Van Der Lugt A, et al. White matter lesion extension to automatic brain tissue segmentation on mri. Neuroimage. 2009;45:11511161.

35. Wolz R, Aljabar P, Hajnal JV, Hammers A, Rueckert D, Initiative AsDN. Leap: Learning embeddings for atlas propagation. Neuroimage. 2010;49:1316-1325.

36. Buckner RL, Head D, Parker J, Fotenos AF, Marcus D, Morris JC, et al. A unified approach for morphometric and functional data analysis in young, old, and demented adults using automated atlas-based head size normalization: Reliability and validation against manual measurement of total intracranial volume. Neuroimage. 2004;23:724-738.

37. Scheltens $P$, Leys D, Barkhof F, Huglo D, Weinstein H, Vermersch $P$, et al. Atrophy of medial temporal lobes on mri in" probable" alzheimer's disease and normal ageing: Diagnostic value and neuropsychological correlates. J Neurol Neurosurg Psychiatry. 1992;55:967-972.

38. Fazekas F, Chawluk JB, Alavi A, Hurtig HI, Zimmerman RA. Mr signal abnormalities at $1.5 t$ in alzheimer's dementia and normal aging. Am J Roentgenology. 1987;149:351-356.

39. Staals J, Makin SD, Doubal FN, Dennis MS, Wardlaw JM. Stroke subtype, vascular risk factors, and total mri brain small-vessel disease burden. Neurology. 2014;83:1228-1234.

40. Grool A, Van der Graaf Y, Mali WTM, Witkamp TD, Vincken K, Geerlings M. Location and progression of cerebral small-vessel disease and atrophy, and depressive symptom profiles: The second manifestations of arterial disease (smart)-medea study. Psychol Med. 2012;42:359-370.

41. Terroni L, Amaro Jr E, losifescu DV, Mattos P, Yamamoto Fl, Tinone G, et al. The association of poststroke anhedonia with salivary cortisol levels and stroke lesion in hippocampal/parahippocampal region. Neuropsychiatr Dis Treat 2015;11:233-242. 
42. Grool AM, Geerlings MI, Sigurdsson S, Eiriksdottir G, Jonsson PV, Garcia ME, et al. Structural mri correlates of apathy symptoms in older persons without dementia ages-reykjavik study. Neurology. 2014;82:1628-1635.

43. Appelman AP, Exalto LG, Van Der Graaf Y, Biessels GJ, Mali WP, Geerlings MI. White matter lesions and brain atrophy: More than shared risk factors? A systematic review. Cerebrovasc Dis. 2009;28:227-242.

44. Kim HJ, Kang SJ, Kim C, Kim GH, Jeon S, Lee JM, et al. The effects of small vessel disease and amyloid burden on neuropsychiatric symptoms: A study among patients with subcortical vascular cognitive impairments. Neurobiol Aging. 2013;34:1913-1920. 


\section{SUPPLEMENTAL DATA}

Data Supplement 1. Visual rating of imaging markers.

Visual rating of symptomatic (acute) and asymptomatic (old) stroke lesions (laterality, type, and number) was performed on T2/FLAIR by an experienced neurologist (JS), who was blinded to the outcome measures.

Lesion type classification. Lesions were classified into cortical / non-lacunar, lacunar and infratentorial lesion type. Cortical / non-lacunar lesions were defined as a lesion involving cortical \pm adjacent subcortical tissue, or large striatocapsular or subcortical lesions $>20 \mathrm{~mm}$ diameter. ${ }^{1}$ Lacunar lesions were defined as rounded or ovoid lesions, $>3 \mathrm{~mm}$ but $<20 \mathrm{~mm}$ diameter, located in the internal or external capsule, nucleus lentiformis, nucleus caudatus, centrum semiovale / white matter, thalamus, or brainstem and carefully distinguished from $\mathrm{WMH}^{2}$ Infratentorial lesions were defined as lesions in the cerebellum or lesions in the brain stem of non-lacunar origin. If no symptomatic lesion was visible on MRI that corresponded with the presenting symptoms, lesion type and laterality were based on the presentation of clinical stroke symptoms if possible.

Visual rating of cerebral microbleeds (CMBs) and lacunes. Presence of CMBs was rated visually by two experienced neurologists (JS and RvO). CMBs were defined as small ( $<5 \mathrm{~mm}$ diameter) homogeneous, round foci of low signal intensity on susceptibility weighted imaging, differentiated from vessel flow voids. ${ }^{3}$ Presence of CMBs was dichotomized in present (if at least one CMB was present) and absent. The interrater Kappa for presence of CMBs was 0.60. The presence of silent lacunes was defined as asymptomatic lacunar lesions $<20 \mathrm{~mm}$.

Visual rating of medial temporal lobe atrophy (MTA). MTA4 was rated visually on T1-weighted images by two independent raters (E.D. and S.S.). with scores ranging from no atrophy (0) to maximum atrophy (4). Right and left hemispheres were rated separately, and the average score of the two independent raters was used. Based on two recent studies that studied practical cut-offs for visual rating scales of atrophy, ${ }^{5,6}$ the following MTA cut-off scores were applied for abnormal atrophy: for patients younger than 75 years an MTA score $\geq 1.5$ and for patients aged 75 years or older an MTA score $\geq 2$. The inter-rater agreement for the MTA scale was moderate to substantial (weighted Kappa7 left MTA: 0.59, right MTA: 0.69).

Total cerebral small vessel disease (cSVD) burden. One point was assigned for a periventricular WMH score of 3 and/or a deep WMH score of at least 2. One point was assigned for presence of enlarged perivascular spaces in the basal ganglia, if these were rated as moderate or extensive ( $\geq 10)$. One point was assigned for 
presence of $\geq 1$ silent lacunes and $\geq 1 \mathrm{CMBs}$ ). This resulted in a total CSVD burden score ranging from 0 to 4 , which was recoded into three categories representing low (score 0-1), medium (score 2), and severe (score 3-4) cSVD burden.

\section{REFERENCES}

1. Wardlaw JM, Smith EE, Biessels GJ, et al. Neuroimaging standards for research into small vessel disease and its contribution to ageing and neurodegeneration. Lancet Neurol 2013;12:822-838.

2. Wardlaw J, Smith C, Dichgans M. Mechanisms of sporadic cerebral small vessel disease: insights from neuroimaging. Lancet Neurol 2013;12:483-497.

3. Wardlaw JM, Smith EE, Biessels GJ, Cordonnier C, Fazekas F, Frayne R, etal. Neuroimaging standards for research into small vessel disease and its contribution to ageing and neurodegeneration. Lancet Neurol. 2013;12:822-838.

4. Scheltens P, Leys D, Barkhof F, Huglo D, Weinstein H, Vermersch $P$, et al. Atrophy of medial temporal lobes on mri in" probable" alzheimer's disease and normal ageing: Diagnostic value and neuropsychological correlates. J Neurol Neurosurg Psychiatry. 1992;55:967-972.

5. Ferreira D, Cavallin L, Larsson EM, Muehlboeck JS, Mecocci P, Vellas B, et al. Practical cut-offs for visual rating scales of medial temporal, frontal and posterior atrophy in alzheimer's disease and mild cognitive impairment. J Intern Med. 2015;278:277-290.

6. Pereira J, Cavallin L, Spulber G, Aguilar C, Mecocci P, Vellas B, et al. Influence of age, disease onset and apoe4 on visual medial temporal lobe atrophy cut-offs. J Intern Med. 2014;275:317-330.

7. Fleiss JL, Cohen J. The equivalence of weighted kappa and the intraclass correlation coefficient as measures of reliability. Edu Psychol Measurement. 1973;33:613-619. 
Supplementary Table 1. Baseline characteristics for total sample, and separate for patients who were included and excluded in the present study

\begin{tabular}{|lccc|}
\hline Variables & $\begin{array}{c}\text { Total sample } \\
(n=250)\end{array}$ & $\begin{array}{c}\text { Included } \\
(n=188)\end{array}$ & $\begin{array}{c}\text { Excluded } \\
(\boldsymbol{n}=62)\end{array}$ \\
\hline Age in years, mean (SD) & $67.5(11.8)$ & $65.4(11.1)$ & $73.9(11.7)^{\mathrm{b}}$ \\
\hline Male sex, $n(\%)$ & $161(64.4)$ & $133(70.7)$ & $28(45.2)^{\mathrm{b}}$ \\
\hline Education, $n(\%)$ & & & \\
$\quad$ Low education & $102(41.1)$ & $68(36.4)$ & $34(55.7)^{\mathrm{a}}$ \\
Middle education & $87(35.1)$ & $70(37.4)$ & $17(27.9)$ \\
High education & $59(23.8)$ & $49(26.2)$ & $10(16.4)$ \\
\hline Time since stroke, mean (SD) & $2.9(0.5)$ & $2.9(0.5)$ & $3.0(0.4)$ \\
\hline Barthel Index, mean (SD) & $19.4(1.5)$ & $19.7(0.9)$ & $18.5(2.4)^{\mathrm{b}}$ \\
\hline History of depression, $n(\%)$ & $55(22.1)$ & $40(21.3)$ & $15(24.6)$ \\
\hline Family history of depression, $n(\%)$ & $34(13.7)$ & $29(15.4)$ & $5(8.3)$ \\
\hline PSD, $n(\%)$ & $28(11.3)$ & $20(10.6)$ & $8(13.3)$ \\
\hline PSA, $n(\%)$ & $42(16.8)$ & $31(16.5)$ & $11(17.7)$ \\
\hline
\end{tabular}

Abbreviations: PSA, post-stroke apathy; PSD, post-stroke depression.

${ }^{a} p<.05,{ }^{b} p<.01$

Supplementary Table 2. Baseline characteristics for total sample, and separate for patients who completed all measurements and patients who dropped out at 1 or 2 time points

\begin{tabular}{|c|c|c|c|}
\hline Variables & $\begin{array}{l}\text { Total sample } \\
\qquad(n=188)\end{array}$ & $\begin{array}{l}\text { Complete } \\
\text { follow-up } \\
(n=174)\end{array}$ & $\begin{array}{l}\text { Drop-outs } \\
(n=14)\end{array}$ \\
\hline Age in years, mean (SD) & $65.4(11.1)$ & $65.2(11.0)$ & $69.1(12.0)$ \\
\hline Male sex, $n(\%)$ & $133(70.7)$ & $123(70.7)$ & $10(71.4)$ \\
\hline \multicolumn{4}{|l|}{ Education, $n(\%)$} \\
\hline Low education & $68(36.4)$ & $60(34.5)$ & $8(61.5)$ \\
\hline Middle education & $70(37.4)$ & $66(37.9)$ & $4(30.8)$ \\
\hline High education & $49(26.2)$ & $48(27.6)$ & $1(7.7)$ \\
\hline Barthel Index, mean (SD) & $19.7(0.9)$ & $19.7(0.9)$ & $19.6(0.7)$ \\
\hline History of depression, $n(\%)$ & $40(21.3)$ & $38(21.8)$ & $2(14.3)$ \\
\hline Family history of depression, $n(\%)$ & $29(15.4)$ & $29(16.7)$ & $0(0.0)$ \\
\hline PSD, $n(\%)$ & $20(10.6)$ & $19(10.9)$ & $1(7.1)$ \\
\hline PSA, $n(\%)$ & $31(16.5)$ & $28(16.1)$ & $3(21.4)$ \\
\hline \multicolumn{4}{|l|}{ Lesion characteristics } \\
\hline Ischemic stroke, $n(\%)$ & $176(93.6)$ & $163(93.7)$ & $13(92.9)$ \\
\hline Left-sided lesion, $n(\%)$ & $80(45.2)$ & $75(45.7)$ & $5(38.5)$ \\
\hline Frontal lesion, $n(\%)$ & $41(33.6)$ & $40(35.4)$ & $1(11.1)$ \\
\hline Basal ganglia lesion, $n(\%)$ & $7(5.1)$ & $7(5.5)$ & $0(0.0)$ \\
\hline \multicolumn{4}{|l|}{ Stroke lesion volume, $n(\%)$} \\
\hline Small & $56(33.3)$ & $50(32.1)$ & $6(50.0)$ \\
\hline Medium & $56(33.3)$ & $51(32.7)$ & $5(41.7)$ \\
\hline Large & $56(33.3)$ & $55(35.3)$ & $1(8.3)$ \\
\hline
\end{tabular}


Supplementary Table 2. continued

\begin{tabular}{|c|c|c|c|}
\hline Variables & $\begin{array}{l}\text { Total sample } \\
\qquad(n=188)\end{array}$ & $\begin{array}{l}\text { Complete } \\
\text { follow-up } \\
(n=174)\end{array}$ & $\begin{array}{l}\text { Drop-outs } \\
(n=14)\end{array}$ \\
\hline \multicolumn{4}{|l|}{ Stroke type, $n(\%)$} \\
\hline Cortical / non-lacunar & $99(56.9)$ & $94(58.4)$ & $5(38.5)^{b}$ \\
\hline Lacunar & $54(31.0)$ & $46(28.6)$ & $8(61.5)^{b}$ \\
\hline Infratentorial & $21(12.1)$ & $21(13.0)$ & $0(0.0)$ \\
\hline \multicolumn{4}{|l|}{ Background damage on MRI } \\
\hline Lacunes and other old infarcts, $n(\%)$ & $75(39.9)$ & $65(37.4)$ & $10(71.4)^{b}$ \\
\hline Cerebral microbleeds, $n(\%)$ & $86(58.5)$ & $80(58.0)$ & $6(66.7)$ \\
\hline ePVS in basal ganglia, $n(\%)$ & $105(56.2)$ & $92(53.2)$ & $13(92.9)^{\mathrm{b}}$ \\
\hline Abnormal MTA left, $n(\%)$ & $10(5.9)$ & $8(5.1)$ & $2(16.7)$ \\
\hline Abnormal MTA right, $n(\%)$ & $10(5.9)$ & $8(5.1)$ & $2(16.7)$ \\
\hline WMH volume (\% ICV) & $0.65(0.97)$ & $0.59(0.92)$ & $1.37(1.31)^{\mathrm{a}}$ \\
\hline $\mathrm{GA}(\% \mathrm{ICV})$ & $0.33(0.04)$ & $0.33(0.04)$ & $0.35(0.03)$ \\
\hline HCV left $\left(\mathrm{mm}^{3}\right)$, mean (SD) & $2752.7(325.8)$ & $2747.5(321.8)$ & $2820.6(382.7)$ \\
\hline HCV right $\left(\mathrm{mm}^{3}\right)$, mean (SD) & $2809.6(366.6)$ & $2802.2(365.4)$ & $2906.6(383.6)^{a}$ \\
\hline \multicolumn{4}{|l|}{ cSVD burden category, $n$ (\%) } \\
\hline $0-1$ & $72(49.0)$ & $71(51.5)$ & $1(11.1)$ \\
\hline 2 & $40(27.2)$ & $36(26.1)$ & $4(44.4)$ \\
\hline $3-4$ & $35(23.8)$ & $31(22.5)$ & $4(44.4)$ \\
\hline
\end{tabular}

Abbreviations: cSVD, cerebral small vessel disease; ePVS, enlarged perivascular spaces; GA, global brain atrophy; HCV, hippocampal volume; PSA, post-stroke apathy; PSD, post-stroke depression; MTA, medial temporal lobe atrophy; $\mathrm{WMH}$, white matter hyperintensities.

Note: Differences were tested between patients with complete follow-up and patients with dropout at one or two time points. Imaging markers were corrected for age, sex, and highest level of education. ${ }^{\mathrm{a}} \mathrm{p}<.10,{ }^{\mathrm{b}} \mathrm{p}<.05$ 
Supplementary Table 3. Odds ratios for the association of imaging markers with post-stroke depression and apathy during the 1-year follow-up period in a sensitivity analysis correcting for presence of old infarcts and lacunes and cognitive impairment

\begin{tabular}{|c|c|c|c|c|}
\hline \multirow{3}{*}{ Parameter } & \multicolumn{2}{|l|}{ PSD } & \multicolumn{2}{|l|}{ PSA } \\
\hline & \multicolumn{2}{|c|}{ Model $1^{a}$} & \multicolumn{2}{|c|}{ Model $2^{b}$} \\
\hline & Adj. OR (95\% Cl) & $P$-value & Adj. OR (95\% Cl) & $P$-value \\
\hline \multicolumn{5}{|l|}{ Lesion characteristics } \\
\hline \multicolumn{5}{|l|}{ Stroke lesion volume } \\
\hline Small & 1.00 (reference) & & 1.00 (reference) & \\
\hline Medium & $0.96(0.34,2.74)$ & .945 & $0.59(0.20,1.72)$ & .334 \\
\hline Large & $0.52(0.20,1.36)$ & .184 & $2.03(0.89,4.65)$ & .093 \\
\hline \multicolumn{5}{|l|}{ Background damage } \\
\hline Lacunes and other old infarcts & $1.04(0.38,2.79)$ & .945 & $0.80(0.37,1.75)$ & .578 \\
\hline $\mathrm{GA}(\% \mathrm{ICV})$ & - & - & $4.76(1.64,13.86)$ & .004 \\
\hline \multicolumn{5}{|l|}{ cSVD burden category } \\
\hline $0-1$ & 1.00 (reference) & & 1.00 (reference) & \\
\hline 2 & $3.04(1.19,7.77)$ & .020 & $1.08(0.45,2.61)$ & .862 \\
\hline $3-4$ & $1.58(0.48,5.16)$ & .450 & $3.90(1.38,10.98)$ & .010 \\
\hline Cognitive impairment & $0.72(0.31,1.71)$ & .865 & $1.59(0.74,3.37)$ & .232 \\
\hline
\end{tabular}

${ }^{a}$ Adjusted for age at baseline, sex, highest level of education, history of depression, stroke volume, old infarcts or lacunes, cognitive impairment, and post-stroke apathy. ${ }^{\mathrm{b}}$ Adjusted for age at baseline, sex, highest level of education, stroke volume, old infarcts or lacunes, cognitive impairment, and poststroke depression.

Abbreviations: CSVD, cerebral small vessel disease; Cl, confidence interval; GA, global brain atrophy; ICV, intracranial volume; OR, odds ratio; PSA, post-stroke apathy; PSD, post-stroke depression.

Supplementary Table 4. Odds ratios for the association of imaging markers with post-stroke depression and apathy during the 1 -year follow-up period in a sensitivity analysis excluding hemorrhagic strokes

\begin{tabular}{|c|c|c|c|c|}
\hline \multirow{3}{*}{ Parameter } & \multicolumn{2}{|c|}{ PSD } & \multicolumn{2}{|l|}{ PSA } \\
\hline & \multicolumn{2}{|c|}{ Model $1^{a}$} & \multicolumn{2}{|c|}{ Model $2^{b}$} \\
\hline & Adj. OR ( $95 \% \mathrm{Cl})$ & $P$-value & Adj. OR $(95 \% \mathrm{Cl})$ & $P$-value \\
\hline \multicolumn{5}{|c|}{ Lesion characteristics } \\
\hline \multicolumn{5}{|c|}{ Stroke lesion volume } \\
\hline Small & 1.00 (reference) & & 1.00 (reference) & \\
\hline Medium & $0.93(0.33,2.59)$ & .884 & $0.83(0.28,2.43)$ & .733 \\
\hline Large & $0.41(0.16,1.05)$ & .065 & $2.48(1.05,5.88)$ & .039 \\
\hline \multicolumn{5}{|c|}{ Background damage } \\
\hline $\mathrm{GA}(\% \mathrm{ICV})$ & - & - & $5.68(2.07,15.60)$ & .001 \\
\hline \multicolumn{5}{|c|}{ cSVD burden category } \\
\hline $0-1$ & 1.00 (reference) & & 1.00 (reference) & \\
\hline 2 & $3.16(1.15,8.70)$ & .026 & $1.06(0.44,2.53)$ & .899 \\
\hline $3-4$ & $1.90(0.56,6.41)$ & .302 & $3.41(1.23,9.47)$ & .019 \\
\hline
\end{tabular}

${ }^{a}$ Adjusted for age at baseline, sex, highest level of education, history of depression, stroke volume, and post-stroke apathy. ${ }^{\mathrm{b} A d j u s t e d}$ for age at baseline, sex, highest level of education, stroke volume, and post-stroke depression.

Abbreviations: cSVD, cerebral small vessel disease; $\mathrm{Cl}$, confidence interval; GA, global brain atrophy; ICV, intracranial volume; OR, odds ratio; PSA, post-stroke apathy; PSD, post-stroke depression. 


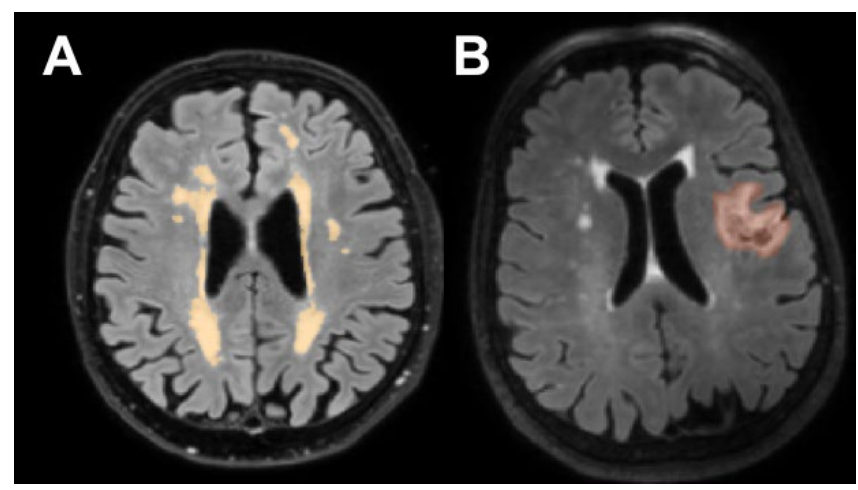

Supplementary Figure 1. Fluid-attenuated inversion recovery (FLAIR) images

(A) Example of a FLAIR image showing the segmented white matter hyperintensities. (B) Example of a FLAIR image showing the segmented stroke lesion.

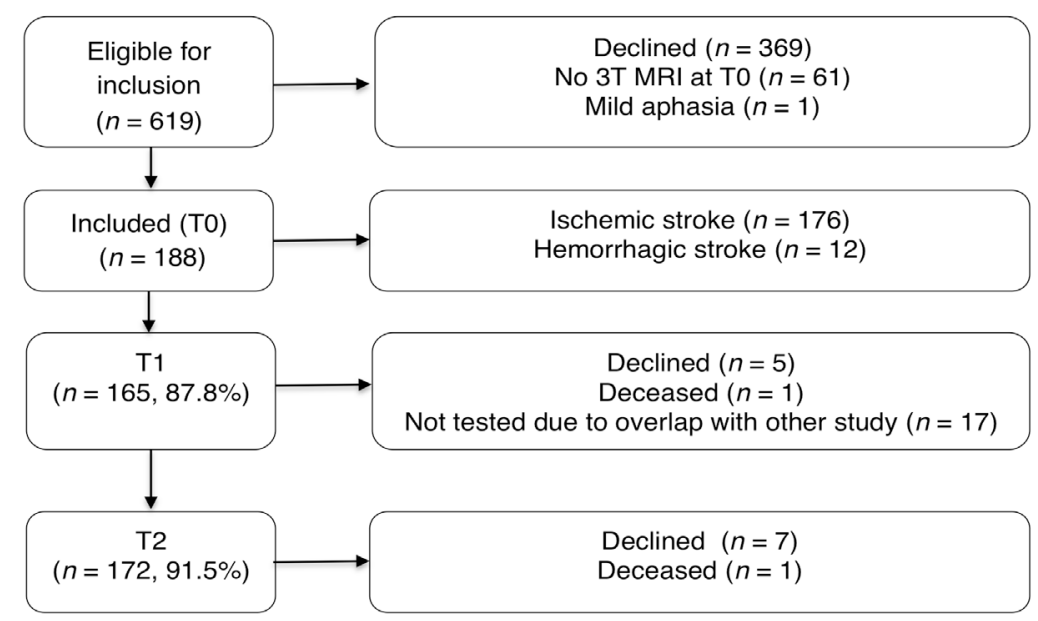

Supplementary Figure 2. Flowchart of inclusion process

Note: Fifteen patients who did not participate at T1, agreed to participate at T2.

Abbreviations: T0 indicates baseline measurement 3 months post-stroke; T1, follow-up 9 months poststroke; T2, follow-up 15 months post-stroke. 


\section{CHAPTER 7}




\title{
BASELINE VASCULAR COGNITIVE IMPAIRMENT PREDICTS THE COURSE OF APATHETIC SYMPTOMS AFTER STROKE: THE CASPER STUDY
}

\author{
Elles Douven \\ Sebastian Köhler \\ Syenna HJ Schievink \\ Robert J van Oostenbrugge \\ Julie Staals \\ Frans RJ Verhey \\ Pauline Aalten
}




\section{ABSTRACT}

Objective: To examine the influence of $\mathrm{VCl}$ on the course of post-stroke depression (PSD) and post-stroke apathy (PSA).

Methods: We included 250 stroke patients, who underwent neuropsychological and neuropsychiatric assessment 3 months after stroke (baseline), and at 6-and 12-month follow-up after baseline. Linear mixed models tested the influence of $\mathrm{VCl}$ in at least 1 cognitive domain (any $\mathrm{VCl}$ ) or multi-domain $\mathrm{VCl}$ ( $\mathrm{VCl}$ in multiple cognitive domains) at baseline, as well as domain-specific $\mathrm{VCl}$ at baseline on levels of depression and apathy over time, with random effects for intercept and slope.

Results: Almost half of the patients showed any $\mathrm{VCl}$ at baseline, and any $\mathrm{VCl}$ was associated with increasing apathy levels from baseline to 12-month follow-up. Patients with multi-domain VCI had higher apathy scores at 6-and 12-month follow-up compared with patients with $\mathrm{VCl}$ in a single cognitive domain. Domain-specific analyses showed that impaired executive function and slowed information processing speed went together with increasing apathy levels from baseline to 6- and 12-month follow-up. None of the cognitive variables predicted the course of depressive symptoms.

Conclusion: Baseline $\mathrm{VCl}$ is associated with increasing apathy levels from baseline to the chronic stroke phase, while no association was found between baseline $\mathrm{VCl}$ and the course of depressive symptoms. Health professionals should be aware that apathy might be absent early after stroke, but may evolve over time in patients with $\mathrm{VCl}$. 


\section{INTRODUCTION}

Apathy and depression are frequent neuropsychiatric symptoms after stroke, with prevalences ranging from 28 to $40 \%,{ }^{1,2}$ and both are associated with poor quality of life ${ }^{3,4}$ and long-term prognosis. ${ }^{5}$ Cross-sectional studies have indicated that vascular cognitive impairment ( $\mathrm{VCl}$ ) is associated with post-stroke depression (PSD), ${ }^{6}$ and according to longitudinal studies $\mathrm{VCl}$ also predicts long-term depressive symptoms. ${ }^{7-10} \mathrm{VCl}$ is frequent after stroke, ranging from mild cognitive impairment to dementia, and is highly age-related with an overall prevalence of $22 \%$ up to 15 years after stroke. ${ }^{11}$

Many studies that have examined the association between VCl and PSD were crosssectional, and classified cognitive impairment based on a screening instrument for global cognition..$^{8,12}$ Only few studies have assessed different cognitive domains. ${ }^{13,14}$ According to a systematic review, including five cross-sectional studies and one prospective study with assessments of multiple cognitive domains, impairments in the domains executive function, memory, language, and processing speed were associated with depression three months post-stroke. ${ }^{15}$ In a six-month follow-up study, patients with visual memory disorders, neglect, and language impairment were at risk to develop depressive symptoms. ${ }^{7}$ One important limitation was that these studies did not consider the role of other neuropsychiatric symptoms like apathy.

Post-stroke apathy (PSA) is a disorder of diminished motivation that shows large overlap with PSD $^{16}$ and is associated with more severe cognitive impairment. ${ }^{17,18}$ According to a meta-analysis of eight cross-sectional and two prospective studies, PSA was associated with lower Mini-Mental State Examination (MMSE) ${ }^{19}$ scores. $^{1}$ Few studies examined the association between PSA and cognitive functioning with a more extensive neuropsychological assessment. ${ }^{17,20}$

Thus, previous studies indicate that both apathy and depression are associated with $\mathrm{VCl}$, but prospective studies with domain-specific information on cognitive impairment are scarce. In addition to any $\mathrm{VCl}$, insight into which specific cognitive domains are involved might be valuable as well, since domain-specific information on cognition has been shown to be a good predictor of other outcomes like longterm cognitive and functional impairment. ${ }^{21,22}$ Therefore, the present study aimed to unravel the relations between $\mathrm{VCl}$, domain-specific cognitive symptoms, PSD and PSA after stroke by examining the influence of any $\mathrm{VCl}$, degree of $\mathrm{VCl}$, and domain-specific $\mathrm{VCl}$ on the course of PSD and PSA over a period of 12-months. 


\section{METHODS}

\section{PATIENT POPULATION}

The Cognition and Affect after Stroke: a Prospective Evaluation of Risks (CASPER) study is a prospective clinical cohort study examining predictors of VCl, PSD, and PSA. The study was approved by the Medical Ethics Committee of Maastricht University Medical Center (MUMC+). Ischemic and hemorrhagic stroke patients who were admitted to the Stroke Unit of MUMC+ or Zuyderland Medical Center (the Netherlands) were approached for participation between June 2013 and August 2015. Stroke was defined as a clinical stroke syndrome. Exclusion criteria were: no written informed consent, insufficient knowledge of the Dutch language, age $<40$, too severe aphasia to understand the study procedure, an Informant Questionnaire on Cognitive Decline in the Elderly (IQ-CODE) score $^{23} \geq 3.60$, MMSE $^{19}$ score $<15$, presence of neurological or psychiatric diseases other than depression that are known to affect cognition, pre-stroke dementia, pre-existing cognitive impairment, mental retardation, blindness, history of stroke less than three years or residual symptoms from previous stroke, and post-surgery stroke / post-anoxic encephalopathy (for details see Douven et al. ${ }^{24}$ ). Eligible patients were enrolled in the study after they signed informed consent.

At baseline (TO); approximately three months post-stroke to avoid confounding effects caused by the acute stroke state), neuropsychological assessment and neuropsychiatric questionnaires were administered by a trained research (neuro)psychologist to the stroke patients according to a standardized protocol, and were repeated at six-month (T1) and 12-month (T2) follow-up. In the present study, only baseline data from the neuropsychological assessment were considered.

\section{NEUROPSYCHOLOGICAL AND NEUROPSYCHIATRIC ASSESSMENT}

The MMSE was administered to assess global cognition. Specific neuropsychological tests were assessed covering three major cognitive domains, i.e. verbal memory, information processing speed, and executive function. The memory domain was measured with the Dutch adaptation of the Rey Verbal Learning Test (immediate and delayed recall). ${ }^{25}$ Information processing speed was measured with the Trail Making Test (TMT) part A, ${ }^{26}$ and the Digit Symbol Substitution Test (DSST), a subtest of the Wechsler Adult Intelligence Scale-III. ${ }^{27}$ Executive function was measured with the TMT part B (mental flexibility) ${ }^{26}$ and the one-minute fluency test (animals and professions categories). ${ }^{25}$ Test scores were converted to z-scores, adjusted for age, sex, and highest level of education based on available norms. ${ }^{27-30}$ Compound scores were calculated for each domain by averaging the z-scores of 
the tests within this domain. For each cognitive domain, patients were classified as no $\mathrm{VCl}$ (compound z-score > -1.0) or VCl (compound z-score $\leq-1.0$ ), based on International Society for Vascular Behavioural and Cognitive Disorders (VASCOG) criteria. ${ }^{31}$ Patients with an impairment in at least one cognitive domain were classified as having any $\mathrm{VCl}$. These patients were further divided in patients with an impairment in multiple cognitive domains (multi-domain $\mathrm{VCl}$ ) and in patients with an impairment in only a single cognitive domain (single-domain $\mathrm{VCl}$ ).

The Montgomery-Åsberg Depression Rating Scale (MADRS), ${ }^{32}$ which ranges from 0 to 60 , was used as outcome measure of depressive symptoms, with a higher score representing a higher level of depressive symptoms. The MADRS was used because it is sensitive to change in symptom severity over time. ${ }^{32}$ The Apathy Evaluation Scale (AES-C) was administered to evaluate the presence and severity of apathy. ${ }^{33}$ Total scores range from 18 to 72, with a higher sum score representing a higher degree of apathy symptoms. The clinician-rated version was used as it has been rated as the most valid apathy instrument. ${ }^{34}$ The IQ-CODE was administered to the informant of the stroke patient to detect possible pre-stroke dementia, and was rated retrospectively (past 5 years prior to the index stroke). ${ }^{23}$

\section{STATISTICAL ANALYSES}

Baseline differences were tested using $X^{2}$ tests for qualitative variables and $t$-tests for quantitative variables, or Mann-Whitney $U$ test if variables were not normally distributed. The influence of any $\mathrm{VCl}$ on the course of depression and apathy was assessed using linear mixed random-effects models, which account for the fact that repeated measurements are correlated within individuals. The models included a random intercept and random slope with an unstructured covariance structure as this resulted in the best fit according to likelihood ratio tests. A group ( $0=$ no $\mathrm{VCl}, 1=$ any $\mathrm{VCl}$ ) by time $(\mathrm{T} 0, \mathrm{~T} 1, \mathrm{~T} 2)$ interaction was added to study differences in rate of change between the groups. In a second set of random-effects models, we examined whether patients with multi-domain $\mathrm{VCl}$ differed from patients with single-domain $\mathrm{VCl}$. Next, analyses were performed to assess the influence of domain-specific $\mathrm{VCl}$ on the course of depression and apathy, using a group (0 = normal cognition, 1 = impaired cognition) by time (T0, T1, T2) interaction. The null hypothesis of no difference in rate of change over time between the groups was tested with a $X^{2}$-test of homogeneity ( 2 degrees of freedom) to see whether there is a significant group-by-time interaction. All analyses were corrected for age at baseline, sex, and highest level of education. Baseline apathy score was added to the model with depression as outcome measure to correct for a possible effect of comorbid apathy on depression, and vice versa. All statistical analyses were performed with Stata version 13.1 for Mac OS X (StataCorp LP, College Station, TX, USA). An alpha level of .05 (two-sided) was used for all analyses. 


\section{RESULTS}

Of the 250 stroke patients initially included at baseline, 4 patients were excluded from the analyses (for 3 patients compound z-scores were missing for all three cognitive domains and for 1 patient the performance on neuropsychological tests was considered unreliable because of mild aphasia). Of the 246 remaining patients, 212 (86\%) patients completed T1. At T2, 13 patients dropped-out (11 refused, two had died) and 19 of the patients who did not participate at T1 re-entered at T2, resulting in 218 (89\%) patients who completed T2 (see Supplementary Figure 1). Patients lost to follow-up were older and had any $\mathrm{VCl}$ more often than patients who completed all measurements (see Supplementary Table 1).

Patient characteristics of the remaining 246 patients included in the analyses are summarized in Table 1, separately for patients with $(n=113)$ and without any VCl $(n=133)$. Patients with any VCl were significantly less educated, had a lower MMSE score, a higher IQ-CODE score, and higher levels of depression and apathy at TO compared with patients without $\mathrm{VCI}($ Table 1). The frequencies of patients performing normal or impaired on the three cognitive domains and the average z-scores on each domain separately are shown in Supplementary Table 2. Compound z-scores for memory, information processing speed, and executive function were missing for 4,1 , and 1 patient(s) at baseline, respectively, as they were cognitively or physically incapable to complete the test(s). These patients were not excluded from the whole study, but only from the particular domain-specific analysis.

\section{INFLUENCE OF BASELINE COGNITIVE IMPAIRMENT ONTHE COURSE OF DEPRESSION}

The results for change in depression score over time according to baseline cognition are shown in Table 2. The overall interaction between cognition group and time was not significant in any of the analyses (Table 2). Also, no differences in rate of decline of depressive symptoms were observed within individual time intervals (T0 to T1 or T0 to T2).

Any $\mathrm{VCl}$. No significant difference in depression score was found between patients with and without any VCl at T0 or at follow-up (Table 2, Figure 1A). Of the patients who had available compound z-scores on all three cognitive domains ( $n=240$ ), we compared patients with single-domain $\mathrm{VCl}(n=58)$ with patients with multi-domain $\mathrm{VCl}(n=49)$. No significant difference in depression score was found between the two groups at T0 or at follow-up (see Table 2, Supplementary Figure 2).

Domain-specific. At T0, no significant difference in depression score was found between patients with or without impairment in memory (Table 2, Figure 2A), information processing speed (Table 2, Figure 2B), or executive function (Table 2, Figure 2C). 
Table 1. Baseline characteristics of the study sample by VCl status

\begin{tabular}{|c|c|c|c|c|c|}
\hline Characteristics & $\begin{array}{l}\text { Total sample } \\
\qquad(n=246)\end{array}$ & $\begin{array}{c}\text { No VCl } \\
(n=133)\end{array}$ & $\begin{array}{l}\text { Any VCl } \\
(n=113)\end{array}$ & $p$ & $\begin{array}{l}\text { t/ Mann- } \\
\text { Whitney U } \\
\text { or } X^{2}(d f)\end{array}$ \\
\hline Age in years, mean (SD) & $67.6(11.9)$ & $66.3(11.4)$ & $69.1(12.2)$ & 0.063 & $-1.869(244)$ \\
\hline Male sex, $n(\%)$ & $158(64.2)$ & $91(68.4)$ & $67(59.3)$ & 0.137 & $2.216(1)$ \\
\hline \multicolumn{6}{|l|}{ Education, $n(\%)$} \\
\hline Low education & $102(41.5)$ & $42(31.6)$ & $60(53.1)$ & & \\
\hline Middle education & $86(35.0)$ & $52(39.1)$ & $34(30.1)$ & $<0.01$ & $12.296(2)$ \\
\hline High education & $58(23.6)$ & $39(29.3)$ & $19(16.8)$ & & \\
\hline Time since stroke in months, mean (SD) & $2.9(0.5)$ & $2.9(0.4)$ & $3.0(0.5)$ & 0.287 & $-1.068(244)$ \\
\hline First-ever stroke, $n(\%)$ & $230(93.5)$ & $125(94.0)$ & $105(92.9)$ & 0.736 & $0.114(1)$ \\
\hline Ischemic stroke, $n(\%)$ & $231(93.9)$ & $128(96.2)$ & $103(91.2)$ & 0.096 & $2.765(1)$ \\
\hline History of depression, $n(\%)$ & $55(22.4)$ & $35(26.3)$ & $20(17.7)$ & 0.106 & $2.613(1)$ \\
\hline Family history of depression, $n(\%)$ & $34(13.9)$ & $20(15.2)$ & $14(12.4)$ & 0.533 & $0.389(1)$ \\
\hline MADRS, mean (SD) & $6.1(5.8)$ & $5.3(5.2)$ & $7.0(6.4)$ & 0.036 & $-2.100^{\mathrm{a}}$ \\
\hline$A E S-C$, mean $(S D)$ & $26.5(8.0)$ & $25.3(7.8)$ & $28.0(8.0)$ & $<0.01$ & $-2.675(244)$ \\
\hline IQ-CODE, mean (SD) & $3.2(0.3)$ & $3.2(0.2)$ & $3.2(0.4)$ & 0.047 & $-1.984^{\mathrm{a}}$ \\
\hline MMSE, mean (SD) & $28.2(1.7)$ & $28.8(1.3)$ & $27.4(1.8)$ & $<0.01$ & $6.637^{a}$ \\
\hline \multicolumn{6}{|l|}{ VCl groups, $n(\%)$} \\
\hline Single-domain $\mathrm{VCl}$ & $58(24.2)$ & $0(0.0)$ & $58(54.2)$ & n.a. & n.a. \\
\hline Multi-domain VCl & $49(20.4)$ & $0(0.0)$ & $49(45.8)$ & n.a. & n.a. \\
\hline Impaired memory & $47(19.4)$ & $0(0.0)$ & $47(43.1)$ & n.a. & n.a. \\
\hline Impaired information processing speed & $78(31.8)$ & $0(0.0)$ & $78(69.6)$ & n.a. & n.a. \\
\hline Impaired executive function & $56(22.9)$ & $0(0.0)$ & $56(50.0)$ & n.a. & n.a. \\
\hline
\end{tabular}

Abbreviations: AES-C, Apathy Evaluation Scale clinician-rated; IQ-CODE, informant questionnaire on cognitive decline in the elderly; MADRS, Montgomery-Åsberg Depression Rating Scale; MMSE, Mini-Mental State Examination; T0, baseline assessment 3 months post-stroke; $\mathrm{VCl}$, vascular cognitive impairment.

Notes: Missing values: 8 for MADRS, 11 for IQ-CODE, 3 for MMSE, 1 for family history of depression, 6 for multi-domain $\mathrm{VCl}, 4$ for impaired memory, 1 for impaired information processing speed, 1 for impaired executive function.

Univariate analyses were performed with $X^{2}$ test for categorical variables and Student's $t$ test (or Mann Whitney $U$ test if applicable) for continuous variables.

a Z-statistic from a Mann-Whitney U Test. 

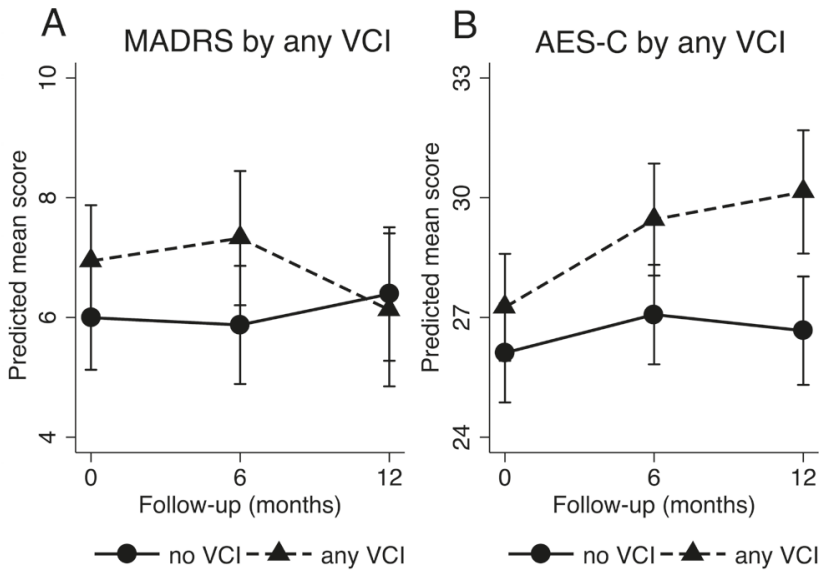

Figure 1. Effect of any vascular cognitive impairment on the course of depression and apathy (A) Course of depression scores by any VCl. (B) Course of apathy scores by any VCl. Based on randomeffects analysis with random intercept, random slope and unstructured correlation matrix, adjusted for age at T0, sex, level of education, group, and group by time interaction. Analyses with MADRS score as outcome were additionally corrected for AES-C score, and vice versa. Predicted mean scores are estimated marginal means for cognition group by time, with all covariates fixed at their means. Error bars represent $95 \%$ confidence intervals around the predicted means. Higher mean scores indicate a higher level of depression and apathy, respectively. AES-C indicates Apathy Evaluation Scale; MADRS, Montgomery-Åsberg Depression Rating; VCl, vascular cognitive impairment.

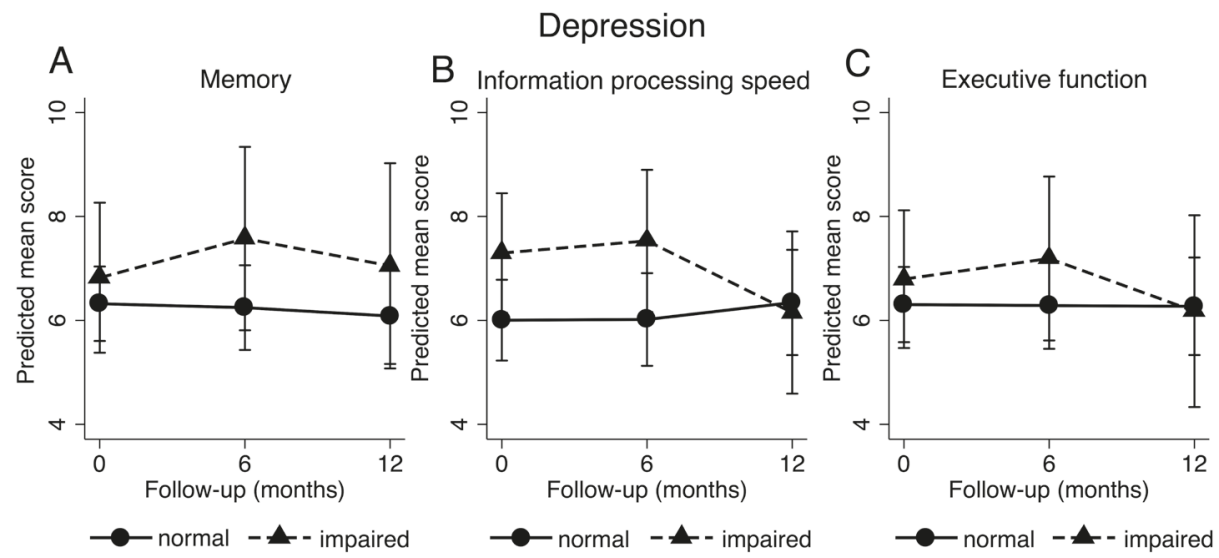

Figure 2. Effect of baseline cognitive performance on the course of depression

(A) Course of depression scores by memory. (B) Course of depression scores by information processing speed. (C) Course of depression scores by executive function. Based on random-effects analysis with random intercept, random slope and unstructured correlation matrix, adjusted for age at T0, sex, level of education, group, and group by time interaction. Analyses were additionally corrected for AES-C score. Predicted mean scores are estimated marginal means for cognition group by time, with all covariates fixed at their means. Error bars represent $95 \%$ confidence intervals around the predicted means. Higher mean scores indicate a higher level of depression. 


\section{INFLUENCE OF BASELINE COGNITIVE PERFORMANCE ON THE COURSE OF APATHY}

The results for change in apathy score over time according to baseline cognition are shown in Table 2. The overall interaction between cognition group and time was not significant in any of the analyses, except for executive function (Table 2). In addition, group differences in rate of change between individual time-intervals were observed.

Any $\mathrm{VCl}$. At T0, the difference in apathy scores between patients with and without any $\mathrm{VCl}$ was not significant (Table 2$)$, but became significant at T1 $(t(245)=2.45$; $p$ $=.015)$ and T2 $(t(245)=3.28 ; p=.001)$, with higher apathy levels in patients with any $\mathrm{VCl}$ (Figure 1B). In line with this, time-stratified analyses showed a significant increase in apathy scores from T0 to T2 in patients with any $\mathrm{VCl}\left(\mathrm{X}^{2}=4.74 ; d f=1 ; p\right.$ $=.030$ ). No significant difference in apathy score was found between patients with multi-domain $\mathrm{VCl}$ and single-domain $\mathrm{VCl}$ at T0 (Table 2), but this group difference became significant at T1 $(t(239)=1.97 ; p=.049)$, and at T2 $(t(239)=2.76 ; p=$ $.006)$, though time-stratified analyses did not reach significance for differences in rate of change from T0 to T2 (see Table 2, Supplementary Figure 2).

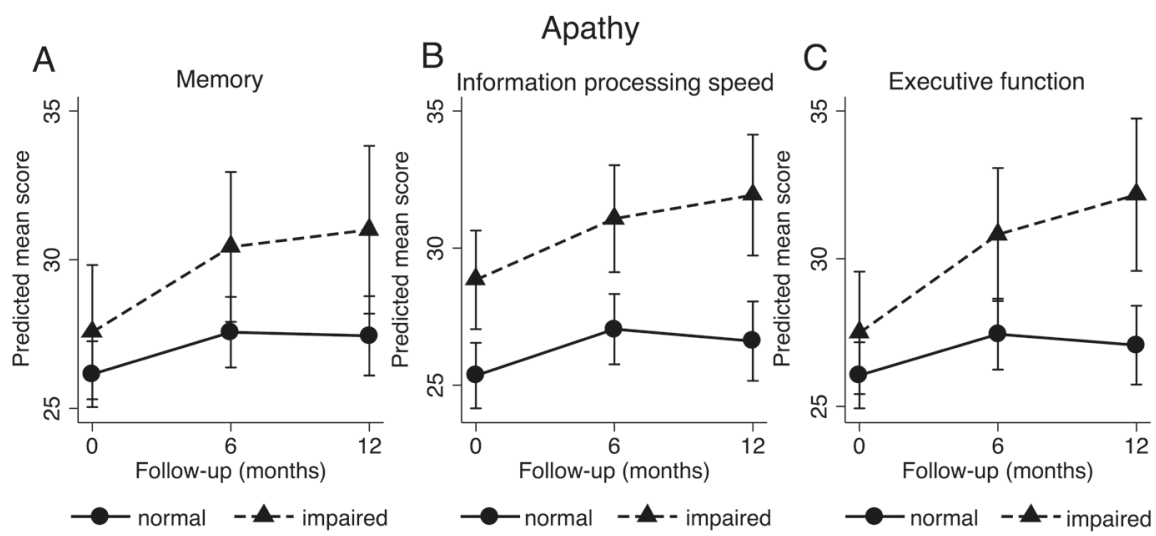

Figure 3. Effect of baseline cognitive performance on the course of apathy

(A) Course of apathy scores by memory. (B) Course of apathy scores by information processing speed.

(C) Course of apathy scores by executive function.

Note: Based on random-effects analysis with random intercept, random slope and unstructured correlation matrix, adjusted for age at T0, sex, level of education, group, and group by time interaction. Analyses were additionally corrected for MADRS score. Predicted mean scores are estimated marginal means for cognition group by time, with all covariates fixed at their means. Error bars represent $95 \%$ confidence intervals around the predicted means. Higher mean scores indicate a higher level of apathy. 


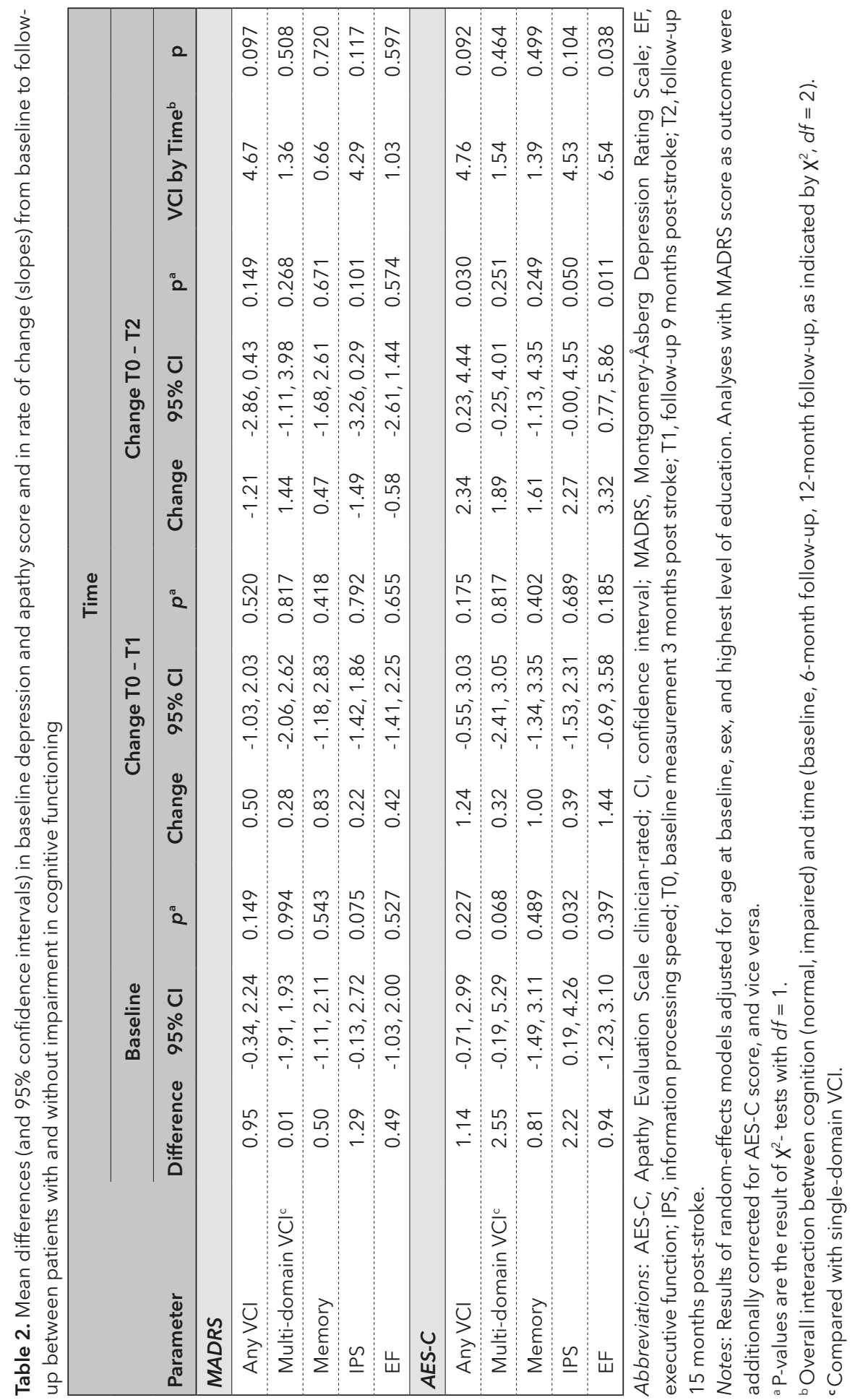


Domain-specific. At T0, no difference in apathy score was found between patients with and without impaired memory performance (Figure 3A), and there were no significant differences in rate of change over time (Table 2). In contrast, a significantly higher apathy score was found in patients with impaired information processing speed at $T 0\left(X^{2}=4.57 ; d f=1 ; p=.033\right.$; Figure 3B, which remained significant at T1 $(t(244)=2.46 ; p=.015)$ and T2 $(t(244)=3.86 ; p<.001)$. Impaired information processing speed at baseline was not associated with increasing apathy scores from T0 to T2 $\left(x^{2}=3.83 ; d f=1 ; p=.0504\right)$. No significant difference in apathy score was found between patients with and without impaired performance on executive function at T0 (Table 2), but patients with impaired performance on executive function showed higher apathy levels at T1 $(t(244)=2.08 ; p=.038)$ and at T2 $(t(244)=3.35 ; p=.001$; Figure $3 C)$. Indeed, the overall interaction between executive function and time on apathy scores was significant, and comparison of the time-specific slopes suggested that this effect was driven by a significant increase in apathy scores from T0 to T2 in patients with impaired executive function (Table 2).

\section{DISCUSSION}

This study examined the association between baseline $\mathrm{VCl}$ and the course of PSD and PSA symptoms over time. The results show that the association between $\mathrm{VCl}$ and course of PSA symptoms varies across time points and with number and type of cognitive domains that are affected. Levels of apathy increased over time in patients with any $\mathrm{VCl}$, and even more when multiple cognitive domains were affected. Change was most pronounced in those with impaired executive functioning and impaired information processing speed. None of the cognitive variables predicted course of depressive symptoms.

The observed association between impaired executive functioning and apathy is in line with previous findings of Almenkerk et al., ${ }^{35}$ who showed that executive dysfunction was associated with apathy in the chronic stroke phase. However, the present study had a prospective design, showing that apathy scores increased from the post-acute to the chronic stroke phase. In addition, the results of the present study are supported by a recent study of Roussel et al. ${ }^{36}$ who reported that half of the stroke patients suffered from a dysexecutive syndrome, including apathy, according to criteria by Godefroy et al. ${ }^{37}$ Similar results were also found in a recent cross-sectional study with lacunar stroke patients, in which apathy, not depression, was associated with impaired executive function and also with impaired information processing speed. ${ }^{38}$ 
In the current study, baseline impairment in information processing speed was associated with higher levels of apathy at baseline, which remained stable throughout time, which is in line with results from the Sydney Stroke Study, ${ }_{1}^{17}$ who also found a significant association between apathy and reduced information processing speed after three to six months. However, they did not provide results on long-term follow-up. As patients with impaired information processing speed already showed higher levels of PSA at baseline, it might be possible that symptoms of PSA have a negative effect on information processing speed as well, or that apathy and impaired information processing speed are both the result of underlying brain pathology, although this had not been examined in earlier studies. No association was found between apathy and memory impairment. However, in patients with $\mathrm{VCl}$ memory is usually less affected compared to impairment in frontal functions like slowed processing speed and executive dysfunction, ${ }^{39}$ and apathy is also considered to be strongly related to frontal lobe dysfunction, ${ }^{40}$ which might explain the lack of association.

The present results suggest that (any, multiple, or domain-specific) $\mathrm{VCl}$ is not associated with the level or course of PSD in the post-acute or chronic stroke phase, which is in contrast with some earlier studies. ${ }^{7,9}$ This discrepancy might be explained by the fact that cognitive impairment was determined in the acute stroke phase in these studies, and also by differences in measurement instruments and criteria for defining cognitive impairment. However, the results of the present study are in agreement with a recent longitudinal study of Bour et al. ${ }^{41}$ that looked into the 1-year course of PSD and also indicated that cognitive deficits at baseline did not predict the development of PSD in the first year post-stroke, though this was only based on the MMSE score. The present results did not show an association between level or course of depression and impaired executive function at three months post-stroke, which was in contrast with earlier studies that indicated that depression and executive function are strongly linked after stroke. ${ }^{13,14}$ Yet, a modest association was found between the course of apathy symptoms over time and executive function, so lack of adjustment for comorbid apathy in these earlier studies could be one explanation for this discrepancy.

The results of the present study support the hypothesis that PSA can develop as an independent syndrome ${ }^{16}$ and may be associated with different risk factors than PSD, as in the present study VCl was only related to the course of apathy. An explanation could be that as apathy is a common behavioral symptom in patients with dementia, it might be more directly related to cognitive dysfunction ${ }^{42}$ than depression, which may be more related to an initial psychological and emotional response to the stressful event of suffering from a stroke. ${ }^{43}$ Neuropsychiatric symptoms after stroke might develop because patients have insufficient cognitive 
capabilities to cope with the consequences of the stroke, as a study with brain injury patients showed that depression and apathy were associated with different coping styles after brain injury. ${ }^{44} \mathrm{VCl}$ might possibly affect coping styles after stroke, thereby influencing the development of neuropsychiatric symptoms. Hence, being aware of the consequences of $\mathrm{VCl}$ in the post-acute and chronic stroke phase, when patients are usually not engaged in rehabilitation care anymore, is important, because not in the early stroke phase (0-3 months post-stroke) but at a later stage (9-15 months), these patients are likely to develop symptoms of PSA. Professional caregivers should be aware of this, as PSA can have a negative impact on quality of life and clinical outcome after stroke. To get a better understanding of how cognitive deficits and neuropsychiatric symptoms affect each other, possibly in a bi-directional manner, the influence of apathy (and also depression) at baseline on long-term cognitive functioning should be studied in future studies as well.

Strengths of our study include the longitudinal design with serial assessments of cognition, depression, and apathy and with relatively low dropout. We used an extensive neuropsychological assessment covering several cognitive domains, and validated instruments were used to measure levels of depression and apathy. A limitation of the study is the exclusion of patients with aphasia and other neurological or psychiatric conditions as this made the study sample less representative of the general stroke population. In addition, of the eligible patients, a substantial part refused to participate and it is possible that particularly these patients may have had depressive or apathetic symptoms, or cognitive complaints. Also, the frequency of any VCl was higher in the patients who droppedout compared with the patients who completed all assessments. Furthermore, it should be noted that there are many reasons for finding an association between $\mathrm{VCl}$ and PSA, not PSD. This could for example be due to differential reliability of the assessments, low prevalence of PSD, or low statistical power. Therefore, the results should be interpreted carefully. Lastly, in some cognitive domains the impaired group was small in sample size, which could have resulted in lack of power in the random-effects models, thereby explaining the fact that we did find significant differences at certain time points, but no significant interaction with time (except for any $\mathrm{VCl}$ and executive function). Thus, the present results showed relatively small but significant differences with respect to the course of apathy between patients with and without VCl after stroke. Nevertheless, these findings are of theoretical interest as they show that the impact of cognitive impairment on the development and course of PSA is likely to be different from PSD, which emphasizes the importance of distinguishing between symptoms of depression and apathy. 


\section{Chapter 7}

In conclusion, $\mathrm{VCl}$ was associated with increasing levels of apathy in the chronic stroke phase, which was strongest for impairments in executive function and information processing speed and for patients with impairments in multiple cognitive domains. Health professionals should be aware that neuropsychiatric symptoms, particularly apathy, might not be present in the early phase but evolve over time in patients with $\mathrm{VCl}$. 


\section{REFERENCES}

1. van Dalen JW, van Charante EPM, Nederkoorn PJ, et al. Poststroke apathy. Stroke 2013; 44:851860

2. Hackett M,Pickles K. Part I. frequency of depression after stroke: an updated systematic review and meta-analysis of observational studies. Int J Stroke 2014;9:1017-1025.

3. Carod-Artal J, Egido J, González J, et al. Quality of life among stroke survivors evaluated 1 year after stroke: experience of a stroke unit. Stroke 2000;31:2995-3000.

4. Kwok T, Lo RS, Wong E, et al. Quality of life of stroke survivors: a 1-year follow-up study. Arch Phys Med Rehabil 2006;87:1177-1182.

5. Hama S, Yamashita H, Shigenobu M, et al. Depression or apathy and functional recovery after stroke. Int J Geriatr Psychiatry 2007;22:1046-1051.

6. Fatoye FO, Mosaku SK, Komolafe MA, et al. Depressive symptoms and associated factors following cerebrovascular accident among Nigerians. J Ment Health 2009;18:224-232.

7. Nys G, Van Zandvoort M, Van Der Worp H, et al. Early cognitive impairment predicts long-term depressive symptoms and quality of life after stroke. J Neurol Sci 2006;247:149-156.

8. Ayerbe L, Ayis $S$, Rudd AG, et al. Natural history, predictors, and associations of depression 5 years after stroke the South London Stroke Register. Stroke 2011;42:1907-1911.

9. Saxena $\mathrm{S}, \mathrm{Ng} \mathrm{T}$, Yong $\mathrm{D}$, et al. Subthreshold depression and cognitive impairment but not demented in stroke patients during their rehabilitation. Acta Neurol Scand 2008;117:133-140.

10. Hachinski V, ladecola C, Petersen RC, et al. National Institute of Neurological Disorders and Stroke-Canadian stroke network vascular cognitive impairment harmonization standards. Stroke 2006;37:2220-2241.

11. Douiri A, Rudd AG, Wolfe CD. Prevalence of Poststroke Cognitive Impairment. Stroke 2013;44:138145.

12. Brodaty $\mathrm{H}$, Withall $\mathrm{A}$, Altendorf $\mathrm{A}$, et al. Rates of depression at 3 and 15 months poststroke and their relationship with cognitive decline: the Sydney Stroke Study. Am J Geriatr Psychiatry 2007; 15:477-486.

13. Pohjasvaara $T$, Leskelä $M$, Vataja $R$, et al. Post-stroke depression, executive dysfunction and functional outcome. Eur J Neurol 2002;9:269-275.

14. Bour A, Rasquin S, Limburg $M$, et al. Depressive symptoms and executive functioning in stroke patients: a follow-up study. Int J Geriatr Psychiatry 2011;26:679-686.

15. Terroni L, Sobreiro MF, Conforto AB, et al. Association among depression, cognitive impairment and executive dysfunction after stroke. Dement Neuropsychol 2012;6:152-157.

16. Withall A, Brodaty $H$, Altendorf $A$, et al. A longitudinal study examining the independence of apathy and depression after stroke: the Sydney Stroke Study. Int Psychogeriatr 2011;23:264-273.

17. Brodaty H, Sachdev PS, Withall A, et al. Frequency and clinical, neuropsychological and neuroimaging correlates of apathy following stroke-the Sydney Stroke Study. Psychol Med 2005;35:1707-1716.

18. Mayo NE, Fellows LK, Scott SC, et al. A longitudinal view of apathy and its impact after stroke. Stroke 2009;40:3299-3307.

19. Folstein MF, Folstein SE, McHugh PR. "Mini-mental state": a practical method for grading the cognitive state of patients for the clinician. J Psychiatr Res 1975;12:189-198.

20. Okada K, Kobayashi S, Yamagata S, et al. Poststroke apathy and regional cerebral blood flow. Stroke 1997; 28:2437-2441.

21. Nys G, Van Zandvoort M, De Kort $P$, et al. The prognostic value of domain-specific cognitive abilities in acute first-ever stroke. Neurology 2005;64:821-827.

22. Galski T, Bruno RL, Zorowitz R, et al. Predicting length of stay, functional outcome, and aftercare in the rehabilitation of stroke patients. The dominant role of higher-order cognition. Stroke 1993;24:1794-1800. 
23. Jorm A. A short form of the Informant Questionnaire on Cognitive Decline in the Elderly (IQCODE): development and cross-validation. Psychol Med 1994;24:145-153.

24. Douven E, Schievink SH, Verhey FR, et al. The Cognition and Affect after Stroke-a Prospective Evaluation of Risks (CASPER) study: rationale and design. BMC Neurol 2016;16:1-11.

25. Lezak MD, Howieson DB, Loring DW, et al. Neuropsychological assessment, 4th edition, 4. Oxford, UK, Oxford University Press, 2004.

26. Reitan RM. Validity of the Trail Making Test as an indicator of organic brain damage. Percept Mot Skills 1958;8:271-276.

27. Wechsler D. Wechsler Memory Scale - 3rd edition: Administration and Scoring Manual, San Antonio, TX, The Psychological Corporation, 1997

28. Schmand B, Houx P, De Koning I. Normen voor stroop kleur-woord tests, trail making test, en story recall van de rivermead behavioural memory test. Nederlands Instituut van Psychologen 2003

29. Van Der Elst W, Van Boxtel MP, Van Breukelen GJ, et al. Rey's verbal learning test: normative data for 1855 healthy participants aged 24-81 years and the influence of age, sex, education, and mode of presentation. J Int Neuropsychol Soc 2005;11:290-302.

30. Van Der Elst W, Van Boxtel MP, Van Breukelen GJ, et al. Normative data for the Animal, Profession and Letter M Naming verbal fluency tests for Dutch speaking participants and the effects of age, education, and sex. J Int Neuropsychol Soc 2006;12:80-89.

31. Sachdev P, Kalaria R, O'Brien J, et al. Diagnostic criteria for vascular cognitive disorders: a VASCOG statement. Alzheimer Dis Assoc Disord 2014;28:206-218.

32. Montgomery SA, Asberg M. A new depression scale designed to be sensitive to change. Brit J Psychiatry 1979;134:382-389.

33. Marin RS, Biedrzycki RC, Firinciogullari S. Reliability and validity of the Apathy Evaluation Scale. Psychiatry Res 1991;38:143-162.

34. Clarke DE, Ko JY, Kuhl EA, et al. Are the available apathy measures reliable and valid? A review of the psychometric evidence. J Psychosom Res 2011;70:73-97.

35. van Almenkerk S, Smalbrugge M, Depla MF, et al. Apathy among institutionalized stroke patients: prevalence and clinical correlates. Am J Geriatr Psychiatry 2015;23:180-188.

36. Roussel M, Martinaud $\mathrm{O}$, Hénon $\mathrm{H}$, et al. The behavioral and cognitive executive disorders of stroke: the GREFEX Study. PloS One 2016;11:1-13.

37. Godefroy $O$, Azouvi $P$, Robert $P$, et al. Dysexecutive syndrome: diagnostic criteria and validation study. Ann Neurol 2010;68:855-864.

38. Lohner V, Brookes RL, Hollocks MJ, et al. Apathy, but not depression, is associated with executive dysfunction in cerebral small vessel disease. PloS One 2017;1-12.

39. Sachdev PS, Brodaty H, Valenzuela M, et al. The neuropsychological profile of vascular cognitive impairment in stroke and TIA patients. Neurology 2004;62:912-919.

40. Tunnard C, Whitehead D, Hurt C, et al. Apathy and cortical atrophy in Alzheimer's disease. Int J Geriatr Psychiatry 2011;26:741-748.

41. Bour A, Rasquin S, Aben I, et al. A one-year follow-up study into the course of depression after stroke. J Nutr Health Aging 2010;14:488-493.

42. Cipriani G, Lucetti C, Danti S, et al. Apathy and dementia. Nosology, assessment and management. J Nerv Ment Dis 2014;202:718-724.

43. Whyte EM, Mulsant BH. Post stroke depression: epidemiology, pathophysiology, and biological treatment. Biol Psychiatry 2002;52:253-264.

44. Finset A, Andersson S. Coping strategies in patients with acquired brain injury: relationships between coping, apathy, depression and lesion location. Brain Inj 2000;14:887-905. 


\section{SUPPLEMENTAL DATA}

Supplementary Table 1. Baseline characteristics for total sample, and separate for patients who completed all measurements and patients who dropped out at 1 or 2 time points

\begin{tabular}{|c|c|c|c|c|c|}
\hline Variables & $\begin{array}{l}\text { All patients } \\
(n=246)\end{array}$ & $\begin{array}{l}\text { Complete } \\
\text { follow-up } \\
(n=222)\end{array}$ & $\begin{array}{l}\text { Dropouts } \\
(n=24)\end{array}$ & $p$ & $t$ or $X^{2}(d f)$ \\
\hline Age (years, SD) & $67.6(11.9)$ & $67.0(11.7)$ & $73.0(12.4)$ & 0.02 & $-2.383(244)$ \\
\hline Male sex $(\%)$ & $158(64.2)$ & $144(64.9)$ & $14(58.3)$ & 0.53 & $0.402(1)$ \\
\hline \multicolumn{6}{|l|}{ Education } \\
\hline Low education, $n(\%)$ & $102(41.5)$ & $88(39.6)$ & $14(58.3)$ & & \\
\hline Middle education, $n(\%)$ & $86(35.0)$ & $79(35.6)$ & $7(29.2)$ & 0.18 & $3.465(2)$ \\
\hline High education, $n(\%)$ & $58(23.6)$ & $55(24.8)$ & $3(12.5)$ & & \\
\hline Time since stroke at TO (months, SD) & $2.9(0.5)$ & $2.9(0.5)$ & $3.0(0.4)$ & 0.91 & $-0.109(244)$ \\
\hline First-ever stroke, $n(\%)$ yes & $230(93.5)$ & $207(93.2)$ & $23(95.8)$ & 0.63 & $0.239(1)$ \\
\hline Lesion type, $n$ (\% ischemic stroke) & $229(93.1)$ & $206(92.8)$ & $23(95.8)$ & 0.68 & $0.173(1)$ \\
\hline History of depression, $n(\%)$ yes & $55(22.4)$ & $49(22.1)$ & $6(25.0)$ & 0.74 & $0.107(1)$ \\
\hline Family depression history, $n(\%)$ yes & $34(13.9)$ & $33(14.9)$ & $1(4.4)$ & 0.17 & $1.929(1)$ \\
\hline IQ-CODE (SD) & $3.2(0.3)$ & $3.2(0.3)$ & $3.2(0.4)$ & 0.93 & $0.084(233)$ \\
\hline MMSE T0 (SD) & $28.2(1.7)$ & $28.2(1.7)$ & $27.6(1.6)$ & 0.09 & $1.683(241)$ \\
\hline Any VCl, $n(\%)$ yes & $113(45.9)$ & $97(43.7)$ & $16(66.7)$ & 0.03 & $4.603(1)$ \\
\hline MADRS T0 (SD) & $6.1(5.8)$ & $6.3(5.9)$ & $4.1(4.7)$ & 0.09 & $1.729(236)$ \\
\hline AES-C TO (SD) & $26.5(8.0)$ & $26.4(8.0)$ & $27.2(8.1)$ & 0.65 & $-0.459(244)$ \\
\hline
\end{tabular}

Abbreviations: AES-C, Apathy Evaluation Scale clinician-rated; IQ-CODE, informant questionnaire on cognitive decline in the elderly; MADRS, Montgomery-Åsberg Depression Rating Scale; MMSE, Mini-Mental State Examination; TO, baseline assessment 3 months post-stroke; $\mathrm{VCl}$, vascular cognitive impairment.

Notes: Missing values: 8 for MADRS, 11 for IQ-CODE, 3 for MMSE, 1 for family history of depression. Univariate analyses were performed with $X^{2}$ test for categorical variables and Student's $t$ test for continuous variables.

Supplementary Table 2. Cognitive functioning at baseline

\begin{tabular}{|lcc|}
\hline Variables & Normal performance & Impaired performance \\
\hline Memory & & \\
\hline$N(\%)$ & $195(80.6)$ & $47(19.4)$ \\
\hline Mean z-score (SD) & $0.45(0.88)$ & $-1.66(0.56)$ \\
\hline Information processing speed & & \\
\hline$N(\%)$ & $189(77.1)$ & $56(22.9)$ \\
\hline Mean z-score (SD) & $-0.10(0.57)$ & $-1.45(0.40)$ \\
\hline Executive function & & \\
\hline$N(\%)$ & $167(68.2)$ & $78(31.8)$ \\
\hline Mean z-score, SD & $-0.05(0.62)$ & $-1.71(0.55)$ \\
\hline
\end{tabular}




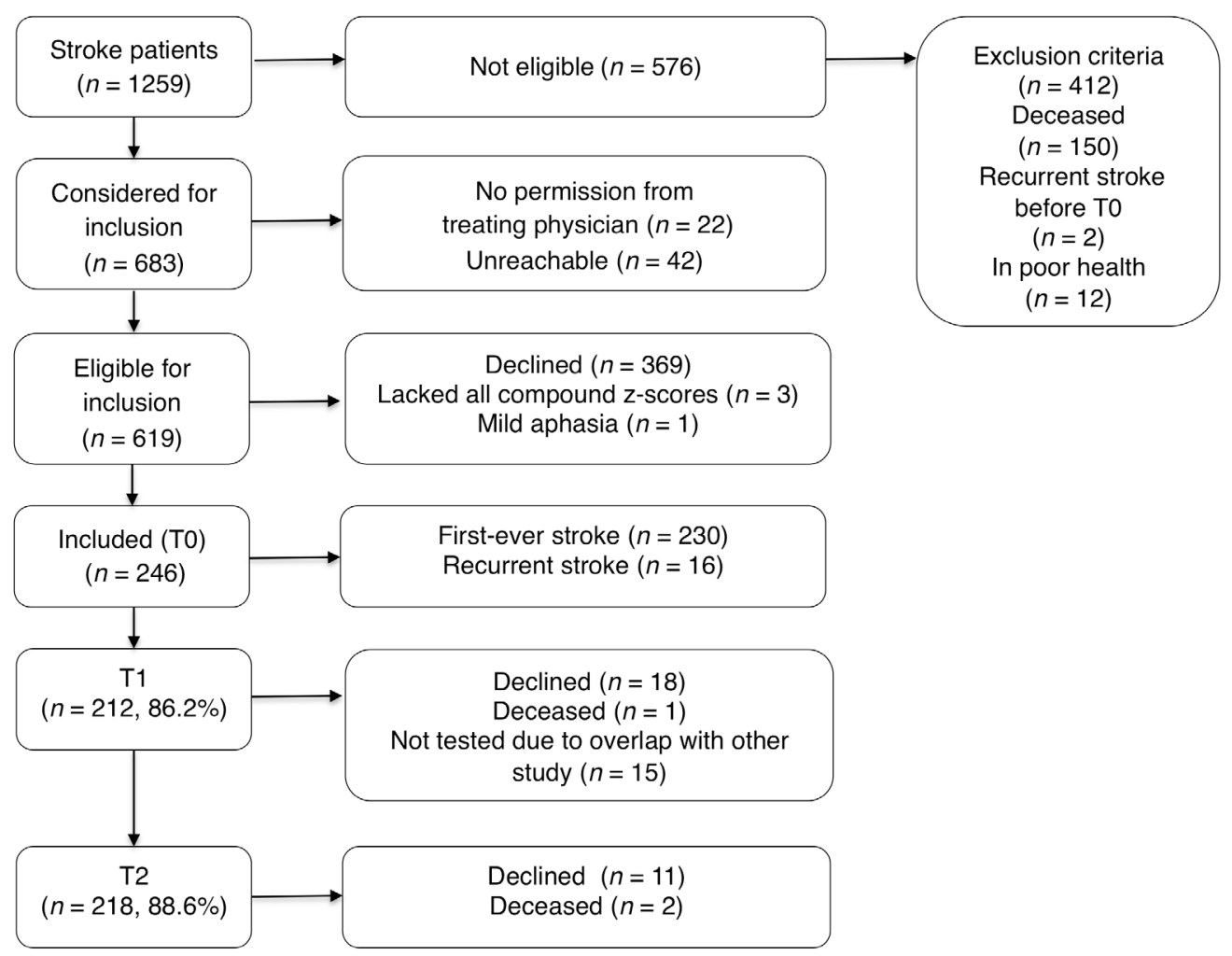

Supplementary Figure 1. Flowchart of inclusion process

Note: Nineteen patients who did not participate at T1, agreed to participate at T2.

Abbreviations: T0, baseline measurement 3 months post stroke; T1, follow-up 9 months post-stroke; T2, follow-up 15 months post-stroke. 


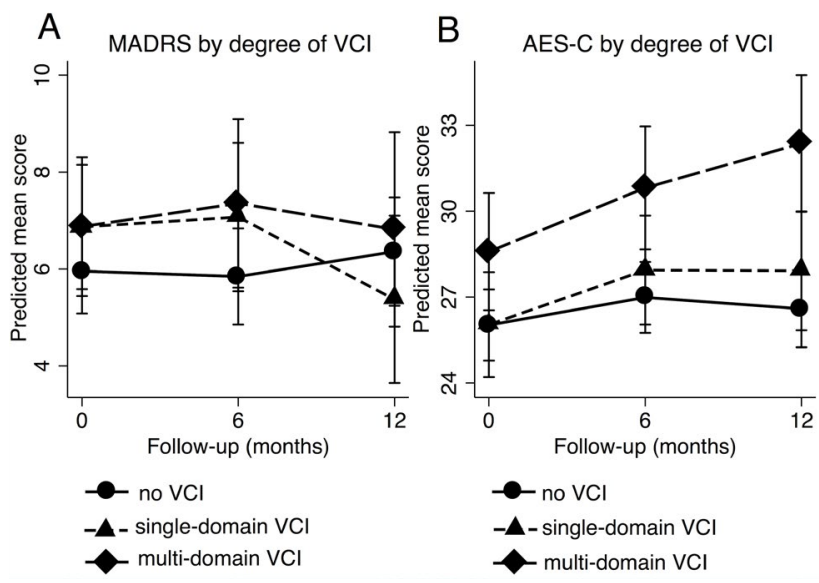

Supplementary Figure 2. Effect of degree of vascular cognitive impairment on the course of depression and apathy

Note: Based on random-effects analysis with random intercept, random slope and unstructured correlation matrix, adjusted for age at T0, sex, level of education, group, and group by time interaction. Analyses with MADRS score as outcome were additionally corrected for AES-C score, and vice versa. Predicted mean scores are estimated marginal means for cognition group by time, with all covariates fixed at their means. Higher mean scores indicate a higher level of depression and apathy, respectively. Abbreviations: AES-C, Apathy Evaluation Scale; MADRS, Montgomery-Åsberg Depression Rating; VCl, vascular cognitive impairment. 


\section{CHAPTER 8}




\section{DEPRESSION-EXECUTIVE DYSFUNCTION SYNDROME AFTER STROKE: RESULTS OF THE CASPER STUDY}

Elles Douven

Pauline Aalten

Julie Staals

Syenna HJ Schievink

Robert J van Oostenbrugge

Frans RJ Verhey

Sebastian Köhler 


\section{ABSTRACT}

Background: Post-stroke depression (PSD) is frequent after stroke, and heterogeneity in etiological factors is found, which results in differences in clinical presentation and prognosis. The co-occurrence of executive dysfunction (ED) and PSD might demarcate the depression-executive dysfunction syndrome (DES). The present study tested whether executive dysfunctions denote a distinct PSD subtype based on neuroimaging correlates and prognosis.

Methods: The present study included 245 stroke patients, who completed neuropsychological and neuropsychiatric assessment at 3 months (TO) poststroke. A subset $(n=186)$ received 3-Tesla brain MRI to evaluate lesionrelated imaging markers, white matter hyperintensity $(\mathrm{WMH})$ volume, global brain atrophy, and total cerebral small vessel disease (cSVD) burden score. Neuropsychological and neuropsychiatric assessments were repeated at 6 and 12-month follow-up after initial testing.

Results: Patients with DES ( $n=55$ ) showed higher WMH volumes compared to all other groups. Patients with DES also showed more frequently old infarcts and higher total cSVD burden compared with PSD-only and patients with neither ED nor PSD, and more global brain atrophy compared with PSD-only. Longitudinal analyses showed that patients with DES had a more chronic course of depressive symptoms relative to the PSD-only group, who improved over time. DES patients also showed a non-improving pattern of worse cognitive performance, which did not differ significantly from the EDonly group.

Conclusions: Patients with DES showed a worse prognosis of depression and cognition and a higher amount of vascular and degenerative brain pathology, suggesting that it denotes a more severe subtype within the PSD spectrum. 


\section{INTRODUCTION}

Post-stroke depression (PSD) occurs in almost a third of stroke survivors, ${ }^{1}$ with a cumulative incidence of $55 \%$ over a period of 15 years after stroke. ${ }^{2}$ Cognitive impairment is also frequent after stroke ${ }^{3}$ and may co-occur with PSD. ${ }^{4}$ The relationship between cognitive impairment and depression appears to be complex, as the direction of the association can go both ways, and they share common risk factors. ${ }^{5}$

It has been suggested that patients who present with both executive dysfunction (ED) and late-onset depression (first manifestation of depression after age 60 or 65 years) show a worse course and clinical presentation, which has been called "depression-executive dysfunction syndrome of late life" (DES). ${ }^{6}$ DES is characterized by persistence of depressive symptoms ${ }^{7}$ and unstable remission. ${ }^{8}$ In a longitudinal study with 116 first-ever stroke patients, stroke patients with DES showed less recovery from ED and depression after 2 years compared with patients with ED only or PSD only, respectively. ${ }^{9}$ Whether DES after stroke also has a distinct course regarding other cognitive domains is unknown. Furthermore, as both PSD ${ }^{10}$ and cognitive impairment ${ }^{11}$ are known to have a negative impact on quality of life $(\mathrm{Q} \circ \mathrm{L})$, the co-occurrence of ED and PSD might be associated with an even worse course of QoL.

With respect to underlying brain pathology, it has been suggested that DES is related to damage in frontal-subcortical brain circuits, ${ }^{6}$ which are known to be involved in mood regulation and executive function. ${ }^{12}$ This was confirmed in a cross-sectional study of 158 stroke patients ${ }^{13}$ which showed that frontal and subcortical lesions were more frequent in the DES-group. Furthermore, DES may be also associated with more widespread brain changes, like higher degree of white matter hyperintensities (WMH), ${ }^{14}$ which are a consequence of cerebral small vessel disease (cSVD). Total cSVD burden score ${ }^{15}$ and generalized brain atrophy ${ }^{16}$ both have been shown to be predictors of decline in executive function. However, whether these imaging markers also play a role in the demarcation of the DES subtype remains to be studied.

The aim of the present 1-year follow-up study was to examine whether ED denotes a distinct subtype of depression after stroke. Our primary aim was to study differences regarding lesion-related and general vascular imaging markers as well as prognostic markers (i.e. course of depression and executive function) between DES, PSD-only, and ED-only groups relative to a comparison group (patients without PSD and ED). Secondary, we examined whether DES was associated with a different course on other cognitive domains (global cognition, memory, and information processing speed) and QoL. 


\section{METHODS}

\section{PATIENT POPULATION}

The present study is a sub-study of the Cognition and Affect after Stroke, a Prospective Evaluation of Risks (CASPER) study, a prospective clinical stroke cohort study, which aims to examine predictors of cognitive impairment, depression, and apathy after stroke. ${ }^{17}$ Patients admitted to the Stroke Unit or out-patient clinic of Maastricht University Medical Center (MUMC+), or Zuyderland Hospital, the Netherlands for a non-fatal ischemic or hemorrhagic stroke between June 2013 and November 2015 were approached for participation. The study was approved by the Medical Ethics Committee of MUMC+ and patients signed informed consent before participation. Exclusion criteria are described in detail elsewhere. ${ }^{17}$

\section{PROCEDURE}

Appointments for baseline measurements (TO) were scheduled around 3 months post-stroke, to avoid interference with acute care and rehabilitation. At T0, patients completed neuropsychological assessment and neuropsychiatric questionnaires. Socio-demographic information was recorded and patients received 3-Tesla (3T) brain Magnetic Resonance Imaging (MRI) if they had no contra-indications and were able to visit the hospital. The neuropsychological assessment and neuropsychiatric questionnaires were repeated at 6-month (T1) and 12-month (T2) follow-up.

\section{MAGNETIC RESONANCE IMAGING}

At baseline, a 3T structural brain MRI was conducted (Philips Achieva, Philips Medical Systems, Best, the Netherlands) The MR protocol consisted of axial T1weighted, T2-weighted, fluid-attenuation inversion recovery (FLAIR), diffusion weighted, and susceptibility weighted sequences (for details see Douven et al. ${ }^{17}$ ). Images were anonymized and analyzed blinded for the patient's neuropsychiatric and cognitive status.

Freesurfer software ${ }^{18}$ was used to segment cortical gray and white matter on T1-weighted scans. WMH volume was segmented on FLAIR images with a semiautomated segmentation tool for $\mathrm{WMH}_{1}{ }^{19}$ using a T1-weighted sequence for anatomical reference and was corrected manually and normalized to intracranial volume (ICV). The ratio of brain parenchymal volume to total ICV was used to measure global brain atrophy and was calculated as 1 minus brain volume. Stroke location, laterality, and presence of old infarcts or lacunes were visually rated 
by an experienced neurologist (JS). The stroke lesion was segmented manually. This volume was normalized to ICV and divided into tertiles (i.e. small, medium, large). Total cSVD burden on MRI was based on a well-validated ordinal scale, ${ }^{20}$ which is based on the counting of presence of $\mathrm{WMH}$, microbleeds, lacunes, and perivascular spaces in the basal ganglia resulting in a total score ranging from 0 to 4 (details can be found in Data Supplement 1).

\section{NEUROPSYCHOLOGICAL ASSESSMENT}

Executive function was measured with the Trail Making Test (TMT) part B (mental flexibility), ${ }^{21}$ the one-minute fluency test (animals and professions categories), ${ }^{22}$ and the Behavioral Assessment of the Dysexecutive Syndrome (BADS) Zoo Map and Key search subtasks. ${ }^{23}$ The MMSE ${ }^{24}$ was used to assess global cognition, episodic verbal memory (immediate and delayed recall) was measured with the Dutch adaptation of the Rey Verbal Learning Test, ${ }^{22}$ and information processing speed was measured with the TMT part $\mathrm{A}^{21}$ and Digit Symbol Substitution Test (DSST). ${ }^{25}$ Test scores of the TMT, DSST, verbal fluency test, and Rey Verbal Learning Test were converted to z-scores, adjusted for age, sex, and highest level of education based on available norms. ${ }^{25-28}$ Raw scores on the BADS subtasks were converted to profile scores ranging from 0 to 4 . ED was defined as a z-score on the TMT-B, verbal fluency animals, or verbal fluency professions <-1.0, or a BADS Zoo Map or Key Search profile score $<1$. For each cognitive domain, compound scores were created by averaging the available $z$-scores of the tests within this domain.

\section{QUESTIONNAIRES}

Patients fulfilling criteria for major or minor depression on the Mini International Neuropsychiatric Interview (M.I.N.I), ${ }^{29}$ or scoring above the cut-off score $\geq 7$ on the Montgomery-Åsberg Depression Rating Scale (MADRS), ${ }^{30}$ were defined as having PSD. In addition, the Barthel Index, ${ }^{31}$ was administered to measure impairment in activities of daily living, and the Stroke Specific Quality of Life (SS-QoL) scale was used to measure health-related QoL. ${ }^{32}$

\section{STATISTICAL ANALYSIS}

Statistical analyses were performed with Stata version 13.1 for Mac OS X (StataCorp, USA). A p-value $<0.05$ (two-sided) was defined as statistically significant. Patients fulfilling criteria for both ED and PSD were grouped in the DES-group. Patients with only ED were grouped in the ED-only group, patients with only PSD were grouped in the PSD-only group, and patients with neither ED nor PSD were grouped in the None-group, which was the reference group in all 
analyses. Baseline differences between subgroups were tested using logistic and linear regression analyses. For the imaging variables, the regression models were corrected for age, sex, and highest level of education. The MADRS score (square root transformation), QoL scores, and MMSE scores (logarithmic transformation) required data transformations to normalize skewed distributions. Linear mixed models were performed to examine whether the course of executive function and depression differed over time from baseline to 12-month follow-up between the subgroups. A group (4 levels: None, ED-only, PSD-only, DES) by time (3 levels: T0, $\mathrm{T} 1, \mathrm{~T} 2)$ interaction was included to study differences in the course of depression, executive function, other cognitive domains, and QoL between the groups. All models included a random intercept and a random slope with an unstructured correlation matrix was added if this gave the best fit according to likelihood ratio tests. The linear mixed models were corrected for age at baseline, sex, and highest level of education.

\section{RESULTS}

Of the 250 participants, 246 completed the MADRS or M.I.N.I and the neuropsychological assessment, and one patient was excluded because of unreliable test results. Of the 245 remaining patients, 211 (86.1\%) patients completed assessments at T1, and 217 (88.6\%) patients completed assessments at T2 (Supplementary Figure 1). Patients lost to follow-up at one or two time points $(n=25)$ did not differ from patients who completed all measurements $(n=220)$ (Supplementary Table 1).

Table 1 shows the baseline characteristics separately for the four groups at T0. Of the 245 patients, 55 (22.5\%) fulfilled criteria for DES, 38 (15.5\%) patients had depressive symptoms but no ED (PSD-only), 88 (35.9\%) patients showed ED but no depressive symptoms (ED-only), and the remaining $64(26.1 \%)$ patients showed neither depressive symptoms nor ED (None).

Differences between the DES group and the other groups with respect to the imaging markers are shown in Table 2. Patients with DES had a higher WMH volume compared with the None group $(F(1,158)=5.37, p=.022)$, ED-only group $(F(1,158)=5.71, p=.018)$, and PSD-only group $(F(1,158)=8.67, p=.004)$. Also, DES patients showed more global brain atrophy compared with the PSD-only group $(F(1,158)=6.15, p=.014)$ and had more frequently old infarcts or lacunes compared with the None group $\left(X^{2}=4.22 ; d f=1 p=.040\right)$ and PSD-only group $\left(X^{2}\right.$ $=5.15 ; d f=2 ; p=.040)$. A higher total cerebral small vessel disease burden score was found in the DES group compared with the None group $(F(1,137)=5.57, p=$ $.020)$ and PSD-only group $(F(1,137)=5.80, p=.017)$. 
Table 1. Baseline characteristics separate for DES subgroup

\begin{tabular}{|lcccc|}
\hline Characteristic & $\begin{array}{c}\text { None } \\
(n=64)\end{array}$ & $\begin{array}{c}\text { ED only } \\
(n=88)\end{array}$ & $\begin{array}{c}\text { PSD only } \\
(n=38)\end{array}$ & $\begin{array}{c}\text { DES } \\
(n=55)\end{array}$ \\
\hline Age (in years), mean (SD) & $65.9(11.0)$ & $69.9(11.4)^{\mathrm{AC}}$ & $64.7(11.3)$ & $67.7(13.5)$ \\
\hline Sex, $n(\%)$ male & $48(75.0)$ & $67(76.1)$ & $15(39.5)^{\mathrm{AB}}$ & $27(49.1)^{\mathrm{AB}}$ \\
\hdashline Low education, $n(\%)$ & $25(39.1)$ & $37(42.1)$ & $13(34.2)$ & $26(47.3)$ \\
Middle education, $n(\%)$ & $23(35.9)$ & $29(33.0)$ & $16(42.1)$ & $18(32.7)$ \\
High education, $n(\%)$ & $16(25.0)$ & $22(25.0)$ & $9(23.7)$ & $11(20.0)$ \\
\hdashline Barthel Index, mean (SD) & $19.8(1.1)$ & $19.4(1.7)$ & $19.6(0.9)$ & $18.9(1.7)^{\mathrm{ABC}}$ \\
\hdashline Pre-stroke depression, $n$ (\%) yes & $12(18.8)$ & $9(10.2)$ & $19(50.0)^{\mathrm{AB}}$ & $15(27.3)^{\mathrm{BC}}$ \\
Family history of depression, $n(\%)$ yes & $5(7.9)$ & $10(11.4)$ & $10(26.3)^{\mathrm{AB}}$ & $9(16.4)$ \\
\hdashline Quality of life (SS-QoL) mean (SD) & $4.4(0.6)$ & $4.3(0.6)$ & $3.8(0.7)^{\mathrm{AB}}$ & $3.4(0.7)^{\mathrm{ABC}}$ \\
\hdashline MMSE score, mean (SD) & $28.8(1.4)$ & $27.8(1.8)^{\mathrm{AC}}$ & $28.8(1.0)$ & $27.5(1.8)^{\mathrm{AC}}$ \\
\hline MADRS, mean (SD) & $2.0(2.0)$ & $2.8(2.0)$ & $11.6(5.0)^{\mathrm{AB}}$ & $11.7(5.5)^{\mathrm{AB}}$ \\
\hline
\end{tabular}

Note: ${ }^{A} p<0.05$ compared with the None group a, ${ }^{B} p<0.05$ compared with the ED-only group, ${ }^{C} p<$ 0.05 compared with the PSD-only group, ${ }^{D} p<0.05$ compared with the DES group.

Abbreviations: DES, depression executive dysfunction syndrome; ED, executive dysfunction; MADRS, Montgomery-Åsberg Depression Rating Scale; MMSE, mini-mental state examination; PSD, post-stroke depression.

Table 2. Imaging characteristics separate for DES subgroup

\begin{tabular}{|c|c|c|c|c|}
\hline Characteristic & $\begin{array}{c}\text { None } \\
(n=54)\end{array}$ & $\begin{array}{l}\text { ED only } \\
(n=68)\end{array}$ & $\begin{array}{l}\text { PSD only } \\
(n=27)\end{array}$ & $\begin{array}{c}\text { DES } \\
(n=37)\end{array}$ \\
\hline \multicolumn{5}{|l|}{ Lesion characteristics } \\
\hline Ischemic stroke, (n (\%) & $50(92.6)$ & $63(92.7)$ & $26(96.3)$ & $35(94.6)$ \\
\hline Left hemispheric stroke, $(n=175), n(\%)$ & $33(54.1)$ & $48(57.8)$ & $21(65.6)$ & $24(43.6)$ \\
\hline Frontal or basal ganglia lesion, $(n=136), n(\%)$ & $14(31.8)$ & $24(47.1)$ & $4(28.6)$ & $10(37.0)$ \\
\hline \multicolumn{5}{|l|}{ Stroke lesion volume, $(n=166), n(\%)$} \\
\hline Small & $12(25.5)$ & $19(32.2)$ & $12(50.0)$ & $12(33.3)$ \\
\hline Medium & 23 (48.9) & $19(32.2)$ & $4(16.7)$ & $10(27.8)$ \\
\hline Large & $12(25.5)$ & $21(35.6)$ & $8(33.3)$ & $14(38.9)$ \\
\hline \multicolumn{5}{|l|}{ Background damage on MRI } \\
\hline Lacunes and other old infarcts, $n(\%)$ & $18(33.3)$ & $29(42.7)$ & $7(25.9)$ & $20(54.1)^{\mathrm{AC}}$ \\
\hline Cerebral microbleeds, $(n=145), n(\%)$ & $24(58.4)$ & $34(65.4)$ & $12(57.1)$ & $15(48.4)$ \\
\hline WMH volume $(\%$ ICV $)(n=166)$, mean (SD) & $0.53(1.03)$ & $0.61(0.79)$ & $0.31(0.43)$ & $0.97(1.17)^{\mathrm{ABC}}$ \\
\hline Global atrophy $(\%$ ICV $)(n=166)$, mean (SD) & $0.33(0.04)$ & $0.33(0.04)$ & $0.31(0.03)$ & $0.34(0.05)^{\mathrm{C}}$ \\
\hline cSVD burden ordinal score $(0-4),(n=145)$, mean (SD) & $1.41(0.97)$ & $1.71(1.30)$ & $1.33(0.91)$ & $1.97(1.45)^{\mathrm{AC}}$ \\
\hline
\end{tabular}

Note: ${ }^{A} p<0.05$ compared with the None group a, ${ }^{B} p<0.05$ compared with the ED-only group, ${ }^{C} p$ $<0.05$ compared with the PSD-only group, ${ }^{\mathrm{D}} p<0.05$ compared with the DES group. The regression models were corrected for age, sex, and highest level of education.

Abbreviations: CSVD, cerebral small vessel disease; DES, depression executive dysfunction syndrome; $E D$, executive dysfunction; ICV, intracranial volume; PSD, post-stroke depression; WMH, white matter hyperintensities. 
Table 3 gives the results for the analyses comparing patients on executive function, depression, memory, information processing speed, and CoL.

Table 3. Mean differences (and 95\% confidence interval) in baseline depression, cognition, and quality of life and in change in depression, cognition, and quality of life (slopes) from baseline between those with Depression Executive Dysfunction Syndrome (DES, $n=55$ ), executive dysfunction only (ED-only, $n=88$ ), post-stroke depression only (PSD-only, $n=38$ ), and those without depression and executive dysfunction at baseline as reference group $(n=64)$

\begin{tabular}{|c|c|c|c|c|c|c|c|}
\hline \multirow[b]{3}{*}{ Parameter } & \multicolumn{6}{|c|}{ Time } & \multirow{3}{*}{ Group xTime } \\
\hline & \multicolumn{2}{|c|}{ Baseline difference } & \multicolumn{2}{|c|}{$\begin{array}{c}\text { Change baseline to } \\
\text { 6-month FU }\end{array}$} & \multicolumn{2}{|c|}{$\begin{array}{l}\text { Change baseline to } \\
\text { 12-month FU }\end{array}$} & \\
\hline & Estimate & $95 \% \mathrm{Cl}$ & Estimate & $95 \% \mathrm{Cl}$ & Estimate & $95 \% \mathrm{Cl}$ & \\
\hline \multicolumn{8}{|l|}{ Depression } \\
\hline ED-only & $0.40^{\star *}$ & $0.12,0.68$ & -0.00 & $-0.44,0.44$ & -0.01 & $-0.54,0.52$ & 0.00 \\
\hline PSD-only & $2.17^{\star \star}$ & $1.82,2.52$ & $-0.80^{* *}$ & $-1.33,-0.25$ & -0.56 & $-1.20,0.08$ & $8.33^{*}$ \\
\hline DES & $2.20^{\star \star}$ & $1.91,2.53$ & -0.47 & $-0.95,0.01$ & -0.36 & $-0.94,0.22$ & 3.70 \\
\hline \multicolumn{8}{|c|}{ Executive function } \\
\hline ED-only & $-0.82^{\star \star}$ & $-1.02,-0.61$ & 0.10 & $-0.07,0.26$ & 0.10 & $-0.07,0.26$ & 1.75 \\
\hline PSD-only & 0.12 & $-0.14,0.37$ & -0.04 & $-0.24,0.16$ & -0.12 & $-0.32,0.09$ & 1.28 \\
\hline DES & $-0.97^{\star \star}$ & $-1.20,-0.74$ & 0.14 & $-0.03,0.32$ & $0.20 *$ & $0.01,0.38$ & 4.63 \\
\hline \multicolumn{8}{|c|}{ Global Cognition } \\
\hline ED-only & $0.14^{\star \star}$ & $0.05,0.23$ & -0.02 & $-0.12,0.08$ & 0.03 & $-0.07,0.13$ & 0.85 \\
\hline PSD-only & 0.03 & $-0.08,0.14$ & 0.00 & $-0.12,0.13$ & -0.01 & $-0.13,0.11$ & 0.03 \\
\hline DES & $0.18^{\star \star}$ & $0.08,0.28$ & 0.02 & $-0.09,0.13$ & 0.02 & $-0.09,0.13$ & 0.25 \\
\hline \multicolumn{8}{|l|}{ Memory } \\
\hline ED-only & $-0.50^{\star \star}$ & $-0.86,-0.12$ & 0.09 & $-0.37,0.37$ & -0.14 & $-0.44,0.16$ & 2.11 \\
\hline PSD-only & 0.14 & $-0.33,0.61$ & -0.00 & $-0.37,0.37$ & 0.02 & $-0.34,0.38$ & 0.02 \\
\hline DES & $-0.88^{\star \star}$ & $-1.30,-0.46$ & -0.06 & $-0.39,0.28$ & -0.21 & $-0.54,0.12$ & 1.57 \\
\hline \multicolumn{8}{|c|}{ Processing speed } \\
\hline ED-only & $-0.49 * \star$ & $-0.76,-0.22$ & -0.02 & $-0.22,0.19$ & -0.15 & $-0.36,0.05$ & 2.55 \\
\hline PSD-only & -0.01 & $-0.35,0.34$ & 0.18 & $-0.08,0.43$ & 0.17 & $-0.08,0.42$ & 2.50 \\
\hline DES & $-0.84^{\star \star}$ & $-1.15,-0.53$ & -0.04 & $-0.27,0.19$ & -0.03 & $-0.26,0.19$ & 0.13 \\
\hline \multicolumn{8}{|c|}{ Quality of life } \\
\hline ED-only & 0.07 & $-0.07,0.21$ & 0.11 & $-0.02,0.24$ & 0.07 & $-0.07,0.21$ & 2.90 \\
\hline PSD-only & $0.45^{\star \star}$ & $0.28,0.63$ & 0.10 & $-0.06,0.26$ & 0.03 & $-0.14,0.20$ & 1.62 \\
\hline DES & $0.64^{\star *}$ & $0.48,0.79$ & 0.02 & $-0.13,0.16$ & 0.05 & $-0.11,0.20$ & 0.35 \\
\hline
\end{tabular}

Note: ${ }^{1} X^{2}$-test ( 2 degrees of freedom) of overall interaction between subgroup and time (baseline, 6-month-up, 12-month follow-up) omnibus test of the null hypothesis of no difference in rate of change over time between subgroups. ${ }^{*} \mathrm{P}<0.05,{ }^{*} \mathrm{P}<0.01$.

Abbreviations: $\mathrm{FU}$, follow-up; $\mathrm{Cl}$, confidence interval; DES, depression executive dysfunction syndrome; ED, executive dysfunction; MADRS, Montgomery-Åsberg Depression Rating Scale; MMSE, Mini Mental State Examination; PSD, post-stroke depression; SS-QoL, stroke-specific quality of life; 


\section{COURSE OF DEPRESSION BY SUBGROUP}

Overall, a significant difference in the course of depressive symptoms was found between the four groups $\left(X^{2}=12.96 ; d f=6 ; p=.044\right)$. The PSD-only group showed a significant decrease in depressive symptoms over time compared with the None group ( $\chi^{2}=8.33 ; d f=2 ; p=.016$ ). In contrast, depressive symptom levels in the DES group remained stable over time compared to the None group $\left(X^{2}=3.70\right.$; $d f=2 ; p=.157$, Figure $1 \mathrm{~A})$.
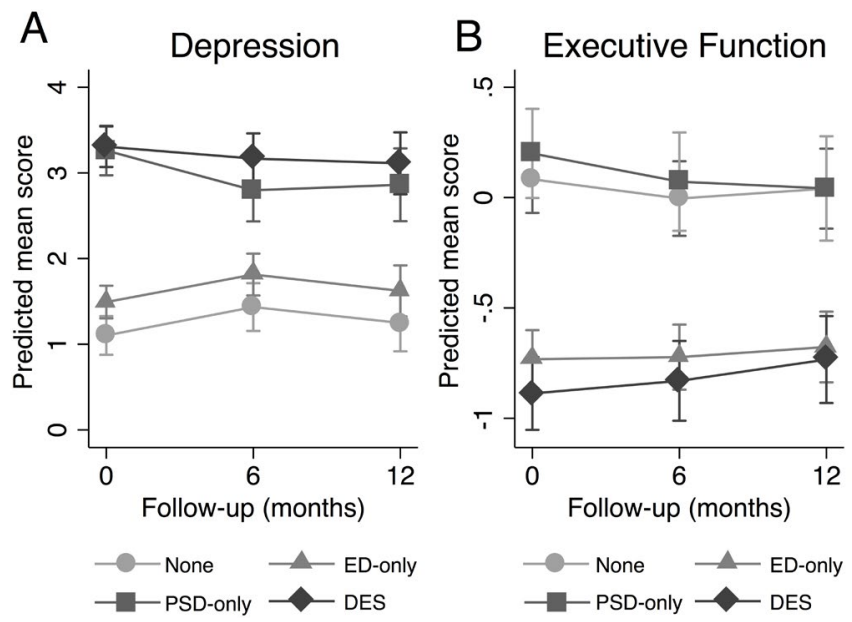

Figure 1. Change over time in executive function and depression scores by subgroup (A) Course of depressive symptoms measured with the MADRS by DES subgroup. (B) Course of executive function performance by DES subgroup.

Note: Based on random-effects analysis adjusted for age at T0, sex, level of education, group, and group by time interaction. Predicted mean scores are estimated marginal means for DES subgroup by time, with all covariates fixed at their means. Higher mean scores indicate a higher level of depression and better performance on executive function, respectively.

\section{COURSE OF EXECUTIVE FUNCTION BY SUBGROUP}

There was a significant difference in performance from T0 to T2 between the four groups $\left(X^{2}=9.63 ; d f=3 ; p=.022\right)$. Overall, performance on executive function in the DES group tended to improve over time relative to the None group ( $X^{2}$ $=4.63 ; d f=2 ; p=.099$, Figure $1 \mathrm{~B})$, and time-stratified analyses showed that this improvement was most notable from T0 to T2 $\left(X^{2}=4.20 ; d f=1 ; p=.040\right)$. Furthermore the PSD-only group showed a significant decrease in performance over time from T0 to T2 relative to the DES group $\left(X^{2}=8.35 ; d f=1 ; p=.004\right)$ and ED-only group $\left(X^{2}=4.54 ; d f=1 ; p=.033\right)$. There were no differences in cognitive trajectories between ED-only and DES, suggesting that these groups had a more or less similar course of performance on executive function over time 
(Figure 1B). Results with respect to differences in performance on the individual test instruments measuring executive function between the subgroups showed that the ED-only group was more likely to show improvement over time compared to the DES group (see TMT-B and TMT-index in Supplementary Table 2).

\section{COURSE OF OTHER COGNITIVE OUTCOMES AND QUALITY OF LIFE BY SUBGROUP}

Global cognition. At TO, patients with DES and ED-only showed lower MMSE scores compared with patients in the None group (Table 2). Overall, no significant difference in the course of MMSE scores was found between the four groups $\left(X^{2}=\right.$ 1.77; $d f=6 ; p=.939$ ), indicating that DES and ED-only scored consistently lower on the MMSE over the course of 12 months (Figure 2A).

Memory. The DES group and ED-only performed significantly worse on memory compared with the None group at T0 (Table 2). Overall, no significant difference in the course of memory performance was found between the four groups $\left(x^{2}=\right.$ 4.09; $d f=6 ; p=.664)$, indicating that DES and ED-only scored lower on memory over the course of 12 months (Figure 2B).

Information processing speed. The DES group and ED-only performed worse on information processing speed compared with the None group at T0 (Table 2). Overall, no significant difference was found between the four groups in the course of performance $\left(x^{2}=8.69 ; d f=6 ; p=.192\right)$, while time-stratified analyses showed a trend for significant differences in the course of performance between the four groups from T0 to T2 $\left(x^{2}=7.40 ; d f=3 ; p=.060\right)$. Further analyses showed that this difference was due to the comparison of trajectories between PSD-only and ED-only, with the former showing a relative improvement in performance from T0 to T2 compared with the ED-only group $\left(x^{2}=7.19 ; d f=1 ; p=.007\right)$. There was no significant difference in cognitive trajectory between the DES group and the None group $\left(X^{2}=0.13 ; d f=2 ; p=.939\right)$, suggesting stable low performance over time in the DES group (Figure $2 \mathrm{C}$ ).

QoL. The SS-QoL score of the DES-group and PSD-only group was significantly lower compared with the None group at T0 (Table 2). Overall, no significant difference was found in the course of QoL between the four groups, $\left(X^{2}=4.67\right.$; $d f=6 ; p=.587$ ), suggesting that QoL scores remained relatively stable in all four groups, with the DES and ED-only groups scoring lower over the course of 12 months (Figure 2D). 

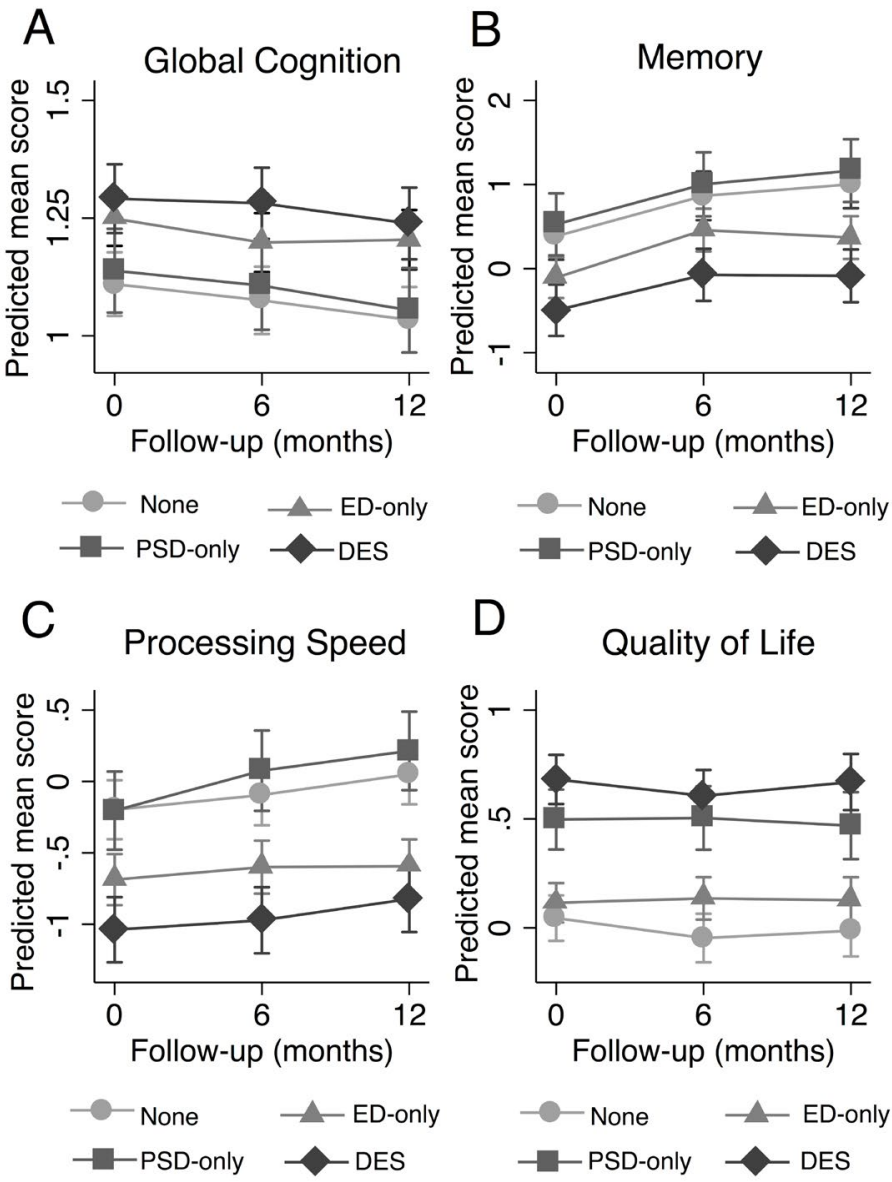

Figure 2. Change over time in performance on global cognition, memory, and processing speed and quality of life scores by subgroup

(A) Course of performance on global cognition measured with the MMSE by DES subgroup. (B) Course of memory performance by DES subgroup. (C) Course of performance on information processing speed by DES subgroup. (D) Course of quality of life scores by DES subgroup.

Note: Based on random-effects analysis adjusted for age at T0, sex, level of education, group, and group by time interaction. Predicted mean scores are estimated marginal means for DES subgroup by time, with all covariates fixed at their means. Higher mean scores indicate better performance on memory, and processing speed. For global cognition and quality of life, higher scores indicate worse performance and worse quality of life, respectively. 


\section{DISCUSSION}

The aim of the present study was to examine whether ED denotes a distinct PSD subtype based on neuroimaging correlates and prognosis. The results of the present study showed that DES is associated with higher WMH volume, more global brain atrophy, higher frequency of old infarcts, and a higher total CSVD burden on MRI. Furthermore, patients with DES showed more functional impairment at baseline, and had a chronic course of depressive symptoms relative to patients with PSD only. DES patients showed a non-improving pattern of worse cognitive performance, which did not differ significantly from the ED-only group.

Depressive symptoms improved in the PSD-only group, whereas they remained stable in the DES-group, suggesting a more chronic course of depression. This is in agreement with earlier findings in patients with late-life depression, ${ }^{8}$ in which ED was associated with relapse and recurrence of depression and residual depressive symptoms. A smaller study by Bour et al, ${ }^{9}$ who studied the relation between depression and ED in 116 first-ever stroke patients also found that DES patients showed a chronic course of depressive symptoms after 2 years.

The DES-group generally showed a stable course of executive function over time, which did not differ significantly from ED-only group. However, results of the individual test instruments showed that the ED-only group was more likely to show improvement over time compared to the DES group. These findings seem to imply, that in general executive function remained impaired in the DES-group. This is consistent with the study by Bour et al., ${ }^{9}$ in which about $50 \%$ of patients with ED-only or DES were still impaired in executive functions after 2 years. However, only presence of ED was evaluated at follow-up, not change in severity over time. On global cognition, information processing speed, and memory, the DES group performed worst, and performance remained stable low over time. QoL scores were lowest in the DES group and remained stable over time as well.

Patients with DES had a significant higher WMH volume compared to all other groups. This is in contrast to the results of the study of Vataja et al., ${ }^{13}$ who could not find an association with white matter changes. Our results seem consistent with findings in depressed geriatric patients, particularly late-onset depression, who tend to show white matter changes in frontal-subcortical circuits. ${ }^{6,33}$ Indeed, it has been shown that WMH in older adults with major depressive disorders relate to more severe impairment in executive functions and memory. ${ }^{34}$ Next to higher WMH volume, patients with DES also had significantly more frequent old infarcts, a higher total CSVD burden score, and more global brain atrophy, compared to patients with PSD-only. These findings suggest that DES is associated with increased generalized (vascular) brain pathology. This provides support for a 
strong biological origin of depression in the DES group compared with the PSDonly group, for which psychosocial factors like a psychological response to the stroke itself might be more involved. ${ }^{35}$ DES therefore seems to denote a subtype of depressed stroke patients with a distinct etiology and poor prognosis, and may need a different treatment approach as some studies found worse response to antidepressant treatment in depressed older adults with executive impairment, ${ }^{36}$ though the evidence base is still weak, ${ }^{37}$ and lacking for DES after stroke.

Important strengths of the present study include the relatively large sample with minimal dropout, the longitudinal design with serial assessments of cognitive and neuropsychiatric functioning, and the use of linear mixed models, which provide the opportunity to look at differences in functioning over time between groups. Yet, some limitations need to be addressed. Patients with pre-stroke cognitive impairment, severe aphasia, and comorbid neurological conditions that are known to affect cognition were excluded from the present study, which may limit the generalizability of our results. Next, imaging markers were only available for a subset of patients, which hampered the possibility to study associations with specific lesion locations. Lastly, the follow-up period was 12 months after initial testing. Although some differences in course over time were found in this mediumterm follow-up, longer follow-up periods might show clearer demarcation of trajectories in cognition and depression. Furthermore, it would allow examining additional outcome parameters such as incident dementia and mortality, as a 12-year follow-up stroke population study indicated that DES was associated with poor long-term survival, ${ }^{38}$ and a population-based cohort study with nondemented elderly found an increased risk for developing dementia in patients with DES over a 9-year follow-up period. ${ }^{39}$

In conclusion, DES seems to denote a subtype of depressed stroke patients with a distinct etiology and poor prognosis. DES might then also ask for a different treatment approach than patients with only PSD. 


\section{REFERENCES}

1. Hackett ML, Pickles K. Part i: Frequency of depression after stroke: An updated systematic review and meta-analysis of observational studies. Int J Stroke. 2014;9:1017-1025.

2. Ayerbe L, Ayis S, Crichton S, Wolfe CD, Rudd AG. The natural history of depression up to 15 years after stroke. Stroke. 2013;44:1105-1110.

3. Jokinen H, Melkas S, Ylikoski R, Pohjasvaara T, Kaste M, Erkinjuntti T, et al. Post-stroke cognitive impairment is common even after successful clinical recovery. Eur J Neurol. 2015;22:1288-1294.

4. Kauhanen M-L, Korpelainen J, Hiltunen P, Brusin E, Mononen H, Määttä R, et al. Poststroke depression correlates with cognitive impairment and neurological deficits. Stroke. 1999;30:18751880.

5. Ayerbe L, Ayis S, Wolfe CD, Rudd AG. Natural history, predictors and outcomes of depression after stroke: Systematic review and meta-analysis. Br J Psychiatry. 2013;202:14-21.

6. Alexopoulos GS, Kiosses DN, Klimstra S, Kalayam B, Bruce ML. Clinical presentation of the "depression-executive dysfunction syndrome" of late life. Am J Geriatr Psychiatry. 2002;10:98106.

7. Alexopoulos GS, Kiosses DN, Heo M, Murphy CF, Shanmugham B, Gunning-Dixon F. Executive dysfunction and the course of geriatric depression. Biol Psychiatry. 2005;58:204-210.

8. Alexopoulos GS, Meyers B, Young R, Kalayam B, Kakuma T, Gabrielle M, et al. Executive dysfunction and long-term outcomes of geriatric depression. Arch Gen Psychiatry. 2000;57:285-290.

9. Bour A, Rasquin S, Limburg M, Verhey F. Depressive symptoms and executive functioning in stroke patients: A follow-up study. Int J Geriatr Psychiatry. 2011;26:679-686.

10. Carod-Artal J, Egido JA, González JL, De Seijas EV. Quality of life among stroke survivors evaluated 1 year after stroke. Stroke. 2000;31:2995-3000.

11. Nys G, Van Zandvoort M, Van Der Worp H, De Haan E, De Kort P, Jansen B, et al. Early cognitive impairment predicts long-term depressive symptoms and quality of life after stroke. J Neurol Sci. 2006;247:149-156.

12. Tekin S, Cummings JL. Frontal-subcortical neuronal circuits and clinical neuropsychiatry: An update. J Psychosom Res. 2002;53:647-654.

13. Vataja R, Pohjasvaara T, Mäntylä R, Ylikoski R, Leskelä $M$, Kalska $H$, et al. Depression-executive dysfunction syndrome in stroke patients. Am J Geriatr Psychiatry. 2005;13:99-107.

14. Alexopoulos GS, Kiosses DN, Choi SJ, et al. Frontal white matter microstructure and treatment response of late-life depression: a preliminary study. Am J Psychiatry. 2002;159:1929-1932.

15. Uiterwijk R, van Oostenbrugge RJ, Huijts M, De Leeuw PW, Kroon AA, Staals J. Total cerebral small vessel disease mri score is associated with cognitive decline in executive function in patients with hypertension. Front Aging Neurosci. 2016;8:1-8.

16. Prins ND, van Dijk EJ, den Heijer T, Vermeer SE, Jolles J, Koudstaal PJ, et al. Cerebral small-vessel disease and decline in information processing speed, executive function and memory. Brain. 2005;128:2034-2041.

17. Douven E, Schievink SH, Verhey FR, van Oostenbrugge RJ, Aalten P, Staals J, et al. The cognition and affect after stroke-a prospective evaluation of risks (casper) study: Rationale and design. BMC Neurol. 2016;16:1-11.

18. Fischl B. Freesurfer. Neuroimage. 2012;62:774-781.

19. De Boer R, Vrooman HA, Van Der Lijn F, Vernooij MW, Ikram MA, Van Der Lugt A, et al. White matter lesion extension to automatic brain tissue segmentation on mri. Neuroimage. 2009;45:11511161.

20. Staals J, Makin SD, Doubal FN, Dennis MS, Wardlaw JM. Stroke subtype, vascular risk factors, and total mri brain small-vessel disease burden. Neurology. 2014;83:1228-1234.

21. Reitan RM. Validity of the trail making test as an indicator of organic brain damage. Percept Mot Skills. 1958;8:271-276. 
22. Lezak MD. Neuropsychological assessment. Oxford University Press, USA; 2004.

23. Wilson B, Alderman N, Burgess P. Behovioural assessment of the dysexecutive syndrome (bads) manual. Bury St Edmonds, England: Thames Valley Test Company. 1996.

24. Folstein MF, Folstein SE, McHugh PR. "Mini-mental state": A practical method for grading the cognitive state of patients for the clinician. J Psychiatr Res. 1975;12:189-198.

25. Wechsler D. Wechsler memory scale - 3rd edition: Administration and scoring manual. San Antonio, TX: The Psychological Corporation; 1997.

26. Schmand B, Houx P, De Koning I. Normen voor stroop kleur-woord tests, trail making test, en story recall van de rivermead behavioural memory test. Nederlands Instituut van Psychologen. 2003.

27. Van Der Elst W, Van Boxtel MP, Van Breukelen GJ, Jolles J. Normative data for the animal, profession and letter $m$ naming verbal fluency tests for dutch speaking participants and the effects of age, education, and sex. J Int Neuropsychol Soc. 2006;12:80-89.

28. Van Der Elst W, Van Boxtel MP, Van Breukelen GJ, Jolles J. Rey's verbal learning test: Normative data for 1855 healthy participants aged 24-81 years and the influence of age, sex, education, and mode of presentation. J Int Neuropsychol Soc. 2005;11:290-302.

29. Overbeek I, Schruers K, Griez E. Mini international neuropsychiatric interview: Nederlandse versie 5.0. 0. DSM-IV [Dutch version]. Maastricht, The Netherlands: Universiteit Maastricht. 1999.

30. Montgomery SA, Asberg M. A new depression scale designed to be sensitive to change. $\mathrm{Br} \mathrm{J}$ Psychiatry. 1979;134:382-389.

31. Mahoney Fl, Barthel DW. Functional evaluation: The Barthel index. Md State Med J. 1965;14:6165.

32. Post MW, Boosman H, Van Zandvoort MM, Passier PE, Rinkel GJ, Visser-Meily JM. Development and validation of a short version of the stroke specific quality of life scale. J Neurol Neurosurg Psychiatry. 2011;82:283-286.

33. Alexopoulos GS, Meyers BS, Young RC, Kakuma T, Silbersweig D, Charlson M. Clinically defined vascular depression. Am J Psychiatry. 1997;154:562-565.

34. Köhler S, Thomas AJ, Lloyd A, Barber R, Almeida OP, O'Brien JT. White matter hyperintensities, cortisol levels, brain atrophy and continuing cognitive deficits in late-life depression. $\mathrm{Br} J$ Psychiatry. 2010;196:143-149.

35. Whyte EM, Mulsant BH. Post stroke depression: Epidemiology, pathophysiology, and biological treatment. Biol Psychiatry. 2002;52:253-264.

36. Baldwin R, Jeffries S, Jackson A, Sutcliffe C, Thacker N, Scott M, et al. Treatment response in late-onset depression: Relationship to neuropsychological, neuroradiological and vascular risk factors. Psychol Med. 2004;34:125-136.

37. McLennan SN, Mathias JL. The depression-executive dysfunction (ded) syndrome and response to antidepressants: A meta-analytic review. Int J Geriatr Psychiatry. 2010;25:933-944.

38. Melkas S, Vataja R, Oksala NK, Jokinen H, Pohjasvaara T, Oksala A, et al. Depression-executive dysfunction syndrome relates to poor poststroke survival. Am J Geriatr Psychiatry. 2010;18:10071016.

39. Vilalta-Franch J, López-Pousa S, Llinàs-Reglà J, Calvó-Perxas L, Merino-Aguado J, Garre-Olmo J. Depression subtypes and 5-year risk of dementia and alzheimer disease in patients aged 70 years. Int J Geriatr Psychiatry. 2013;28:341-350. 


\section{SUPPLEMENTAL DATA}

Data Supplement 1. Total cerebral small vessel disease (cSVD) burden score

The total cSVD burden score is based on counting the presence of microbleeds, lacunes, perivascular spaces in the basal ganglia, and extensive white matter hyperintensities (WMH) on MRI. ${ }^{1}$ One point was assigned for visual rating of extensive WMH (JS), which was defined as a periventricular WMH Fazekas score of 3 and/or a deep WMH Fazekas score of at least $2 .{ }^{2}$ Presence of microbleeds was rated visually on susceptibility weighted imaging by two experienced neurologists (JS and RvO). Microbleeds were defined as small ( $<5 \mathrm{~mm}$ diameter) homogeneous, round foci of low signal intensity, differentiated from vessel flow voids. ${ }^{3}$ One point was assigned for the presence of $\geq 1$ microbleed(s). The presence of silent lacunes was defined as asymptomatic lacunar lesions $<20 \mathrm{~mm}^{3}$ and one point was assigned for presence of $\geq 1$ silent lacune(s). For perivascular spaces, one point was assigned if these were visually rated (JS) as moderate or extensive $(\geq 10)$ in the basal ganglia. Summing of the scores for each cSVD marker resulted in a total cSVD burden score ranging from 0 to 4 . The original CSVD burden scale was slightly adapted here, as in contrast with the original CSVD scale, we used susceptibility weighted imaging (SWI) for counting microbleeds.

\section{REFERENCES}

1. Staals J, Makin SD, Doubal FN, Dennis MS, Wardlaw JM. Stroke subtype, vascular risk factors, and total mri brain small-vessel disease burden. Neurology. 2014;83:1228-1234

2. Fazekas $\mathrm{F}$, Chawluk JB, Alavi A, Hurtig HI, Zimmerman RA. Mr signal abnormalities at $1.5 t$ in alzheimer's dementia and normal aging. Am J Roentgenology. 1987;149:351-356

3. Wardlaw JM, Smith EE, Biessels GJ, Cordonnier C, Fazekas F, Frayne R, etal. Neuroimaging standards for research into small vessel disease and its contribution to ageing and neurodegeneration. Lancet Neurology. 2013;12:822-838 
Supplementary Table 1. Baseline characteristics for total sample, and separate for patients who completed all measurements and patients who dropped out at 1 or 2 time points

\begin{tabular}{|lccc|}
\hline Variables & $\begin{array}{c}\text { All patients } \\
(n=245)\end{array}$ & $\begin{array}{c}\text { Complete } \\
\text { follow-up } \\
(n=220)\end{array}$ & $\begin{array}{c}\text { Dropouts } \\
(n=25)\end{array}$ \\
\hline Age (in years), mean (SD) & $67.5(11.9)$ & $66.9(11.6)$ & $73.6(12.5)^{\mathrm{b}}$ \\
\hline Male sex, $n(\%)$ & $157(64.1)$ & $143(65.0)$ & $14(56.0)$ \\
\hline Low education, $n(\%)$ & $101(41.2)$ & $86(39.1)$ & $15(60.0)$ \\
Middle education, $n(\%)$ & $86(35.1)$ & $79(35.9)$ & $7(28.0)$ \\
High education, $n(\%)$ & $58(23.7)$ & $55(25.0)$ & $3(12.0)$ \\
\hline Barthel Index, mean (SD) & $19.4(1.5)$ & $19.5(1.4)$ & $19.0(1.7)$ \\
\hline Pre-stroke depression, $n(\%)$ & $55(22.5)$ & $49(22.3)$ & $6(24.0)$ \\
\hline Family history of depression, $n(\%)$ & $34(13.9)$ & $33(15.0)$ & $1(4.2)$ \\
\hline Quality of life (SS-QoL) mean (SD) & $4.1(0.7)$ & $4.1(0.7)$ & $4.0(0.9)$ \\
\hline MMSE score, mean (SD) & $28.1(1.7)$ & $28.2(1.7)$ & $27.6(1.6)$ \\
\hline MADRS, mean (SD) & $6.1(5.8)$ & $6.3(5.9)$ & $4.4(4.8)$ \\
\hline
\end{tabular}

Note: ${ }^{\mathrm{a}} p<.05,{ }^{\mathrm{b}} p<.01$.

Abbreviations: MADRS, Montgomery-Åsberg Depression Rating Scale; MMSE, Mini-Mental State Examination; SS-QoL, Stroke-Specific Quality of Life.

Supplementary Table 2. Mean differences (and 95\% confidence interval) in executive function and in change in executive function (slopes) from baseline between those with Depression Executive Dysfunction Syndrome (DES, $n=55$ ), executive dysfunction only (ED-only, $n=88$ ), post-stroke depression only (PSD-only, $n=38$ ), and those without depression and executive dysfunction at baseline as reference group $(n=64)$ on individual tests

\begin{tabular}{|c|c|c|c|c|c|c|c|}
\hline \multirow[b]{3}{*}{ Parameter } & \multicolumn{6}{|c|}{ Time } & \multirow[b]{3}{*}{ Group xTime } \\
\hline & \multicolumn{2}{|c|}{ Baseline difference } & \multicolumn{2}{|c|}{$\begin{array}{c}\text { Change baseline to } \\
\text { 6-month FU }\end{array}$} & \multicolumn{2}{|c|}{$\begin{array}{l}\text { Change baseline to } \\
\text { 12-month FU }\end{array}$} & \\
\hline & Estimate & $95 \% \mathrm{Cl}$ & Estimate & $95 \% \mathrm{Cl}$ & Estimate & $95 \% \mathrm{Cl}$ & \\
\hline \multicolumn{8}{|c|}{ Executive function } \\
\hline \multicolumn{8}{|c|}{ Verbal fluency - animals } \\
\hline ED-only & $-0.77^{\star \star}$ & $-1.01,-0.53$ & -0.16 & $-0.39,0.07$ & 0.03 & $-0.23,0.29$ & 3.02 \\
\hline PSD-only & 0.02 & $-0.29,0.33$ & -0.16 & $-0.44,0.12$ & -0.07 & $-0.38,0.24$ & 1.30 \\
\hline DES & $-1.02^{\star *}$ & $-1.30,-0.75$ & 0.21 & $-0.05,0.46$ & $0.34^{*}$ & $0.05,0.62$ & 5.54 \\
\hline \multicolumn{8}{|l|}{ TMT-B } \\
\hline ED-only & $-0.94^{\star *}$ & $-1.26,-0.63$ & $0.41^{\star \star}$ & $0.11,0.70$ & 0.25 & $-0.05,0.56$ & $7.49^{\star}$ \\
\hline PSD-only & 0.13 & $-0.27,0.53$ & -0.00 & $-0.36,0.35$ & -0.13 & $-0.49,0.23$ & 0.65 \\
\hline DES & $-0.98^{\star \star}$ & $-1.33,-0.62$ & -0.02 & $-0.34,0.31$ & 0.13 & $-0.20,0.46$ & 0.84 \\
\hline \multicolumn{8}{|l|}{ TMT-index } \\
\hline ED-only & $0.64^{\star \star}$ & $0.32,0.96$ & $-0.50^{\star \star}$ & $-0.87,-0.13$ & $-0.51^{\star \star}$ & $-0.88,-0.14$ & $9.96^{\star \star}$ \\
\hline PSD-only & -0.07 & $-0.47,0.34$ & 0.12 & $-0.33,0.57$ & 0.37 & $-0.07,0.81$ & 2.86 \\
\hline DES & 0.21 & $-0.16,0.58$ & -0.05 & $-0.45,0.36$ & -0.02 & $-0.42,0.39$ & 0.06 \\
\hline
\end{tabular}

Note: ${ }^{1} X^{2}$-test ( 2 degrees of freedom) of overall interaction between subgroup and time (baseline, 6-month-up, 12-month follow-up) omnibus test of the null hypothesis of no difference in rate of change over time between subgroups.

Abbreviations: $\mathrm{FU}$, follow-up; $\mathrm{Cl}$, confidence interval; $\mathrm{DES}$, depression executive dysfunction syndrome; $E D$, executive dysfunction; PSD, post-stroke depression; TMT-B, trail making test part $B$; TMT-index, trail making test index part. ${ }^{*} P<0.05,{ }^{*} \mathrm{P}<0.01$. 


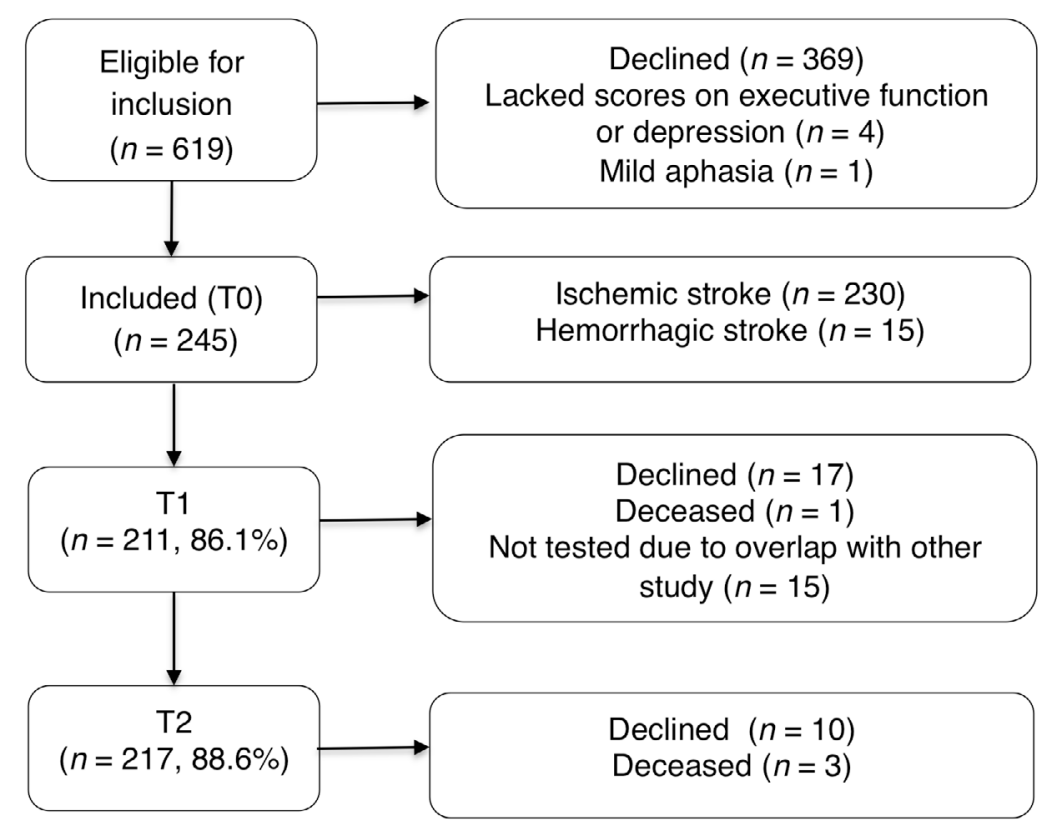

Supplementary Figure 1. Flowchart of inclusion process

Note: Nineteen patients who did not participate at T1, agreed to participate at T2.

Abbreviations: T0 indicates baseline measurement 3 months post stroke; T1, follow-up 9 months poststroke; T2, follow-up 15 months post-stroke. 
Depression-executive dysfunction syndrome post stroke 


\section{CHAPTER 9}


GENERAL DISCUSSION 
Chapter 9 


\section{GENERAL DISCUSSION}

Post-stroke depression (PSD) and post-stroke apathy (PSA) are frequently occurring neuropsychiatric syndromes after stroke, with estimated mean prevalences of $31 \%{ }^{1}$ and $35 \%,{ }^{2}$ respectively. Over the past years, many studies have been conducted to study the underlying factors involved in the etiology of PSD, while only few studies have been conducted to unravel the etiological factors involved in the development of PSA. Despite a large overlap in clinical symptoms between PSD and PSA, some studies found evidence for differences in associated biological and psychosocial factors, suggesting that these two concepts might, at least to some extent, represent independent syndromes. However, few studies looked at the development, course, and associated factors of both syndromes at the same time in a longitudinal study. Therefore, we investigated the association between several biological and psychosocial risk factors and the development and course of PSD and PSA. In particular, we focused on potential similarities and differences between these two syndromes. A better understanding of how these risk factors differentially influence the development and course of PSD and PSA is not only important from a conceptual point of view, but may also result in better recognition, and earlier detection of these conditions. Of particular interest, a better understanding may lead to a better diagnostic distinction between PSD and PSA. Consequently, this may lead to better treatment and a better clinical prognosis after stroke.

The Cognition and Affect after Stroke: a Prospective Evaluation of Risks (CASPER) study, a one-year prospective clinical stroke cohort follow-up study $(n=250)$ was performed (Chapter 3 ) to answer the following research questions. We examined whether there is evidence for a bi-directional relationship between post-stroke fatigue (PSF) and PSD, and PSF and PSA (Chapter 4). In addition, we studied the influence of premorbid personality traits (Chapter 5 ) and vascular cognitive impairment ( $\mathrm{VCI}$ ) (Chapter 7) on the development and course of PSD and PSA. A systematic review and meta-analysis was conducted (Chapter 2) to examine associations between imaging markers and PSD and PSA and we studied the role of stroke lesion-related and more generalized brain imaging markers in the development of PSD and PSA in the CASPER cohort (Chapter 6). Furthermore, we examined whether the co-occurrence of executive dysfunction and PSD, the so-called "Depression-Executive Dysfunction Syndrome" (DES), denotes a distinct PSD subtype based on neuroimaging correlates and prognosis (Chapter 8).

First, in this chapter the main findings and conclusions of this thesis are presented and methodological considerations are addressed. Furthermore, conceptual and clinical implications and recommendations for future research are discussed. 


\section{MAIN FINDINGS}

\section{Imaging markers}

The neurobiology of PSD has received much attention in the past decades, and focused mainly on associations with lesion laterality (i.e. the 'lesion location hypothesis' of PSD, stating that PSD is associated with left frontal lesions). Neurobiological markers of PSA have been studied less frequently compared with PSD. In Chapter 2, we performed a systematic literature review and meta-analysis to examine the association between PSD or PSA and stroke lesion laterality, type, and location, also stratified by study phase (i.e. acute, post-acute, chronic). Associations with other imaging markers (e.g. white matter hyperintensities $(\mathrm{WMH})$, atrophy, lesion volume, number of lesions, cerebral microbleeds) were reviewed qualitatively to provide an up-to-date overview. We found that lesions in the frontal lobe or basal ganglia were associated with increased odds of PSD in the post-acute (0.5 - 6 months) stroke phase. PSA was associated with hemorrhagic stroke in the acute phase (< 15 days), whereas in the post-acute phase it was associated with ischemic stroke. However, as the number of included PSA studies was limited, no firm conclusions could be drawn. Therefore, large cohort studies using sophisticated imaging techniques and considering a broad range of imaging markers in association with both PSD and PSA are needed.

With respect to the underlying neurobiology of PSD and PSA, focus has shifted from individual lesion locations to extent of damage to neuronal networks being involved in the development of PSD and PSA, with evidence for different subcircuits associated with PSD ${ }^{3}$ and PSA. ${ }^{4}$ This damage can arise from single stroke lesions, but also as a consequence of more generalized brain damage such as WMH, lacunes, and microbleeds. These are markers that occur as a consequence of cerebral small vessel disease (cSVD) and can be identified on magnetic resonance imaging (MRI). In Chapter 6 the association between lesion-related imaging markers and background markers of vascular and degenerative pathology and the development of PSD and PSA was examined in the CASPER cohort including 250 patients with recent stroke. The results showed that after correction for stroke lesion volume, global brain atrophy and severe cSVD burden, but not stroke lesion-related markers, were associated with the development of PSA in the first year post-stroke, independent of co-morbid PSD. After correction for stroke lesion volume and history of depression, global brain atrophy and medium cSVD burden were associated with the development of PSD. After correction for comorbid PSA, only medium cSVD remained significantly associated with PSA. This suggests that atrophy and increased CSVD burden are important predictors for the development of PSA, and, less strongly, for PSD. In general, these findings indicate 
that particularly cumulative background brain damage is involved in developing neuropsychiatric symptoms after stroke, and not the location or volume of the stroke lesion itself, with a stronger involvement in PSA than for PSD. A possible underlying mechanism could be that the cumulative brain pathology results in damage to microstructural networks, which are involved in the regulation of mood and motivational processes.

\section{Fatigue}

Post-stroke fatigue (PSF) is a frequent complaint after stroke and several crosssectional studies found an association between PSF and PSD. ${ }^{5}$ However, few longitudinal studies have examined this association, therefore little is known about the directionality of this relationship. As there is large overlap in symptoms between PSA and PSD, ${ }^{6}$ and both frequently co-occur, 7,8 PSA might be related to PSF as well, though this relationship has not been studied yet. A better understanding of the inter-relationships between PSD, PSA, and PSF is important, as incorporation of this information may eventually lead to better treatment strategies for these syndromes.

In Chapter 4 we examined the temporal relationships between PSF and PSD and between PSF and PSA, by measuring the effect of PSF at three months post-stroke on the course of depressive and apathetic symptoms over a period of 12-month follow-up. In addition, we studied the reverse direction by examining the effect of PSD (or PSA) at three months post-stroke on the course of fatigue symptoms. Since the relationship between PSF and PSD is likely to be influenced by multiple other factors, ${ }^{9}$ we controlled for sleep apnea, high neuroticism, and history of depression in our analyses. The results showed a bi-directional association between PSF and PSD, whereas PSA was not associated with PSF. Levels of PSF and PSD remained stable over time. Therefore, a timely detection of these conditions in stroke patients is important, as they tend to persist. As there are currently few treatment options available to treat or relieve PSF per se, ${ }^{10}$ a potential treatment strategy is to focus on treating PSD, which might also have a beneficial effect on (the prevention of) PSF.

\section{Premorbid personality traits}

Previous studies in the general population found personality traits, and particularly high neuroticism, to be an important predictor of onset and course of neuropsychiatric symptoms. ${ }^{11,12}$ However, associations with personality have been less frequently studied in a stroke population. In Chapter 5 we found an association between high neuroticism and high levels of depression in 245 stroke patients at baseline (three months post-stroke), and this figure remained stable during the 12-month follow-up. In contrast, low extraversion was associated with 
high levels of apathy at baseline, and this association remained stable over time as well. Since different personality traits were associated with PSD and PSA, the results are in line with the suggestion that PSA can develop as an independent syndrome from PSD, having its own associated risk factors.

Although previous studies already showed that high neuroticism is associated with PSD, ${ }^{13,14}$ the present study adds to this that high neuroticism is a prognostic factor in determining the course of PSD symptoms over time. We controlled for level of apathy, as apathy might mediate the relationship between neuroticism and PSD by influencing emotion regulation ${ }^{15}$ and causing social inhibition. ${ }^{16}$ The results implicate that pre-stroke factors, like premorbid personality, may act as potential vulnerability factors to develop PSD or PSA and are associated with the sustainability of PSD and PSA. Possibly, personality traits may influence the way patients cope with the stressful event of suffering from a stroke, ${ }^{17}$ which is important to take into account during treatment and rehabilitation.

\section{Vascular cognitive impairment}

Vascular Cognitive Impairment (VCl) is frequent after stroke, with an overall prevalence of $22 \%$ up to 15 years after stroke. ${ }^{18} \mathrm{VCl}$ can be due to (strategic) infarcts, but is often mixed with AD pathology. ${ }^{19}$ According to VASCOG criteria, ${ }^{20}$ $\mathrm{VCl}$ can be defined as an impairment in one or more cognitive domains, with a clear temporal relationship between a vascular event (e.g. acute stroke) and the cognitive impairment, preferably with neuroimaging evidence of stroke or other vascular pathology. Therefore, to ensure the vascular etiology of cognitive impairment, stroke patients with pre-stroke cognitive impairment or dementia were excluded in the CASPER study.

Although many studies found an association between $\mathrm{VCl}$ and PSD ${ }^{21,22}$ or PSA, ${ }^{23}$ these often defined cognitive impairment based on a global cognitive screening instrument, rather than studying associations with different cognitive domains. Also, prospective studies studying the influence of $\mathrm{VCl}$ on the course of PSD and PSA are scarce. In Chapter 7 we performed longitudinal analyses to examine the

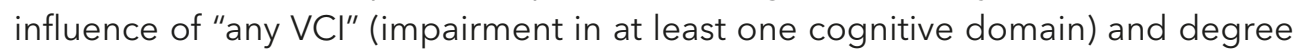
of $\mathrm{VCl}$ (impairment in multiple cognitive domains versus impairment in a single domain) on the course of depression and apathy symptoms over time. Any $\mathrm{VCl}$ predicted an increase in apathy levels from three to 15 months post-stroke, which was most pronounced in patients with $\mathrm{VCl}$ in multiple cognitive domains (particularly executive dysfunction and slowed information processing speed). None of the cognitive variables predicted the course of depressive symptoms. These results implicate that although apathy symptoms may be absent in the first months after stroke, these symptoms can develop over time in patients with VCl. 
Health professionals should be aware of this risk, as apathy is associated with a worse clinical prognosis. ${ }^{2}$

\section{Depression-executive dysfunction syndrome as a subtype of PSD}

Heterogeneity in clinical presentation and prognosis of PSD might be due to differences in etiological factors and subtypes of PSD may exist. In Chapter 8 we examined whether executive dysfunctions (ED) denote a distinct PSD subtype by studying whether the co-occurrence of ED and PSD in a depression-executive dysfunction depression subtype (DES) is associated with different clinical correlates and a worse prognosis. In a subset $(n=186)$ that received 3 Tesla structural brain $\mathrm{MRI}$, we found that the DES-subtype was associated with higher WMH volume, a higher frequency of old infarcts, more global brain atrophy, and higher total cSVD burden on MRI at three months post-stroke. Longitudinal analyses in 245 stroke patients showed that DES was associated with a less benign course, including a non-improving pattern of worse cognitive performance compared with ED-only and higher, and chronic high levels of depression compared with PSD-only. Our findings suggest that DES is associated with increased generalized (vascular) brain pathology, which provides support for a strong biological origin of depression in the DES group compared with the PSD-only group, for which psychosocial factors like a psychological response to the stroke itself might be more involved. ${ }^{24,25}$ Thus, DES might denote a subtype of depressed stroke patients with a distinct etiology and poor prognosis compared with patients with PSD only, and may therefore ask for a different treatment approach.

\section{EVIDENCE FOR INDEPENDENCE OF PSD AND PSA}

Factors associated with PSD and PSA in this thesis are summarized in Figure 1. Global atrophy, low extraversion, and $\mathrm{VCl}$ in multiple cognitive domains, particularly executive dysfunction and slowed information processing speed, were all associated with the development of PSA. Patients with $\mathrm{VCl}$ in multiple domains also showed an increase in apathy levels, but not depressive levels, over time during the one-year follow-up. Lesions in the frontal lobe or basal ganglia, high levels of fatigue, and high neuroticism were specifically associated with the development of PSD. Levels of depression remained stable from baseline to follow-up. As we controlled for the overlap in symptomatology between PSD and PSA in our analyses, the factors in Figure 1 are specifically associated with PSD and PSA, respectively and enable us to differentiate between these two syndromes. However, a phenotypical overlap exists between these syndromes, as some patients presented with both PSD and PSA. Figure 2 shows the independent occurrence of PSD and PSA and its overlap in the CASPER cohort for each time 


\section{Chapter 9}

point after stroke. The number of patients with independent PSA is rather small, though these numbers are dependent on how we defined PSD and PSA, which also differs greatly in previous literature, as there is no consensus.

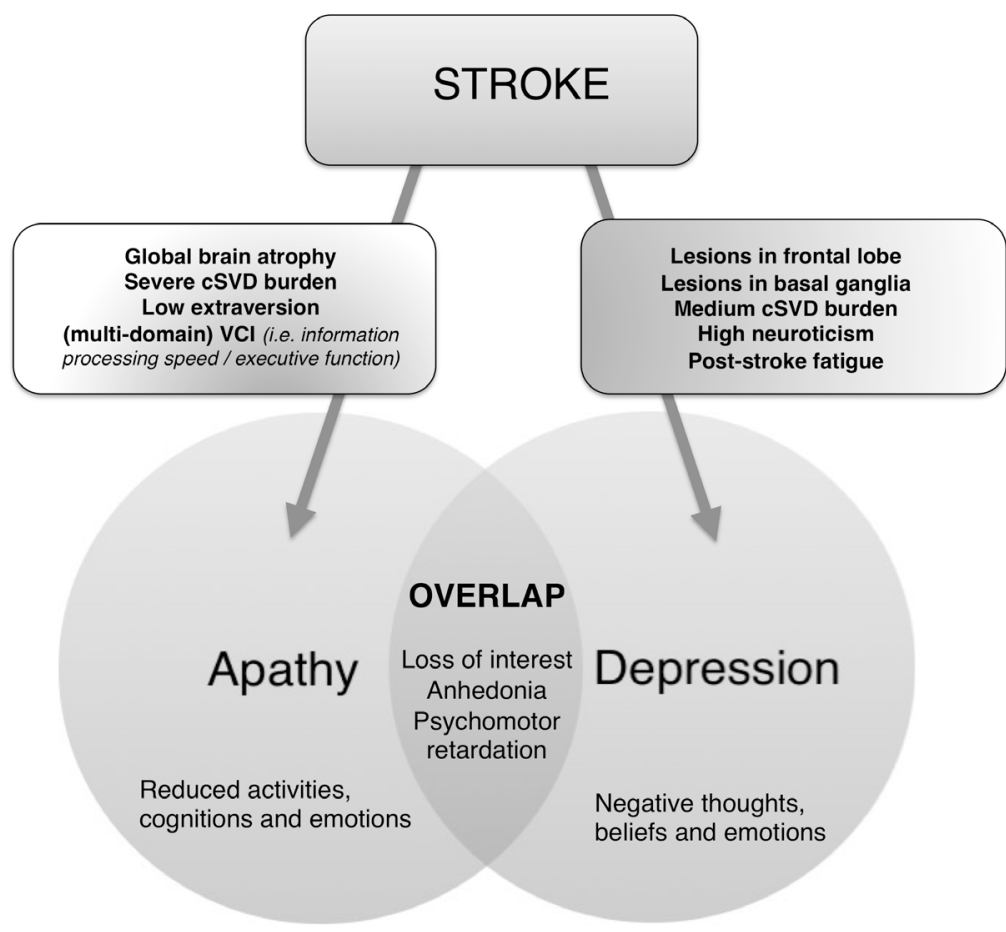

Figure 1. Factors specifically involved in the development of post-stroke apathy and depression Abbreviations: cSVD, cerebral small vessel disease; $\mathrm{VCl}$, vascular cognitive impairment.

\section{DEFINING PSD AND PSA}

In Figure 2 and in Chapters 4 and 6, we defined PSA as a score $\geq 37$ on either the clinician-rated Apathy Evaluation Scale (AES) ${ }^{26}$ or informant-rated AES. The AES is a frequently used measure for apathy in the stroke population, and is evaluated to be a psychometrically robust measure for apathy. ${ }^{27}$ It has a good discriminant validity as it correlates low with measures of depression and anxiety. ${ }^{26,28}$ At three months post-stroke, a PSA frequency of almost $17 \%$ was found in our cohort, increasing to almost $27 \%$ at six-month follow-up, and $23 \%$ at 12 -month follow-up.

The frequency for PSA in CASPER is somewhat lower than the estimated mean prevalence of $34.6 \%$ reported in the meta-analysis by van Dalen and colleagues. ${ }^{2}$ 
This may be due to the fact that the patients in our cohort were relatively mild to moderately affected by the stroke, as patients with severe stroke were more severely impaired and therefore not eligible to participate or it was too burdensome for them. Also, patients with severe aphasia and pre-stroke cognitive impairment were excluded, which may have affected the frequency of PSA as well.

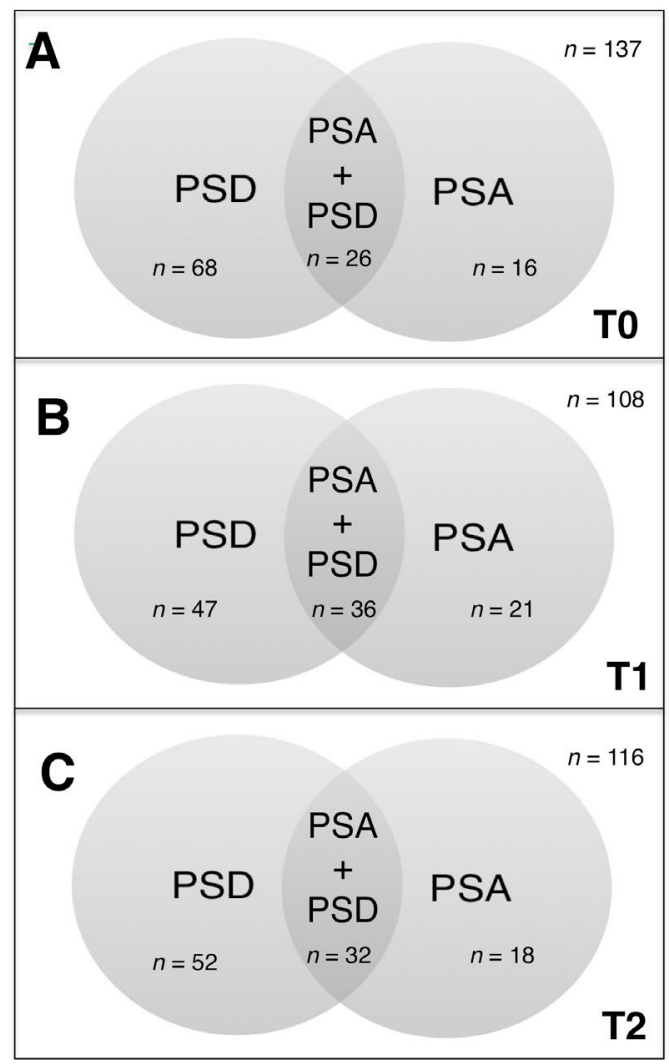

Figure 2. Overlap and independence of PSD and PSA at 3, 9, and 15 months post-stroke Abbreviations: PSA, post-stroke apathy; PSD, post-stroke depression; T0, 3 months post-stroke; T1, 9 months post-stroke; T2, 15 months post-stroke.

PSD was defined as a score $\geq 7$ on the Montgomery-Åsberg Depression Rating Scale (MADRS) ${ }^{29}$ or a diagnosis of major depressive disorder or minor depression on the Mini International Neuropsychiatric Interview (M.I.N.I.) ${ }^{30}$ (Figure 2 and Chapter 4). Although both the Hospital Anxiety and Depression Scale (HADS-D) Depression subscale, ${ }^{34}$ and MADRS are valid screening instruments for PSD as they focus less on somatic symptoms, ${ }^{31}$ we chose to use the MADRS over the HADS-D as outcome measure for depressive symptoms, as the MADRS is a clinician-rated questionnaire, which is especially designed to be sensitive to change. ${ }^{29}$ 
In the CASPER cohort, a PSD frequency of $38 \%$ was found at three months poststroke, which remained stable overtime (39\% at six-month and 12-month follow-up). This frequency is somewhat higher compared with the estimated mean prevalence of $31 \%$ reported in the meta-analysis by Hackett and Pickles. ${ }^{1}$ Studies included in the meta-analysis either applied diagnostic criteria according to Diagnostic and Statistical Manual of Mental Disorders DSM third-edition revised (DSM-III-R), DSMIV, or DSM-5 using a (semi-)structured interview (e.g. M.I.N.I, ${ }^{32}$ Structured Clinical Interview for DSM disorders (SCID) ${ }^{33}$ ), or they defined PSD as a score above a cutpoint on a self-rated or clinician-rated depression scale (e.g. MADRS, ${ }^{29}$ HADS-D, ${ }^{34}$ Beck Depression Inventory, ${ }^{35}$ Geriatric Depression Scale ${ }^{36}$ ), with different cut-offs applied for the same instrument between studies. The diversity in instruments and cut-off scores and also the time of measurement is likely to have affected the frequencies found in each study. In CASPER, we applied relatively mild criteria for PSD (Chapter 4) with respect to the MADRS score, resulting in a heterogeneous group of PSD patients with mild to severe depression.

We examined the influence of risk factors (e.g. severe fatigue, personality traits, $\mathrm{VCl}, \mathrm{DES}$ ) on the level and course of depressive symptoms by looking at change in the continuous MADRS scores (Chapter 4, 5, 7, and 8), rather than whether it influenced the categorization of patients as either depressed or non-depressed, so the difficulty of applying correct criteria to define PSD did not influence our results. For PSA, we looked at the influence of risk factors (i.e. severe fatigue, personality traits, $\mathrm{VCl}$ ) on the level and course of apathy symptoms by examining change in the continuous AES scores on the clinician-rated AES (Chapter 4, 5, and 7). We chose to use the clinician-rated AES over the informant-rated AES, as the former was shown to have the highest reliability. ${ }^{27}$ However, an important disadvantage of using continuous scales is that it is unclear whether a significant increase or decrease on the scale is also clinically relevant. Particularly for patients who cannot be defined as being depressed or apathetic, it is questionable whether it matters if these patients show higher or lower scores on a scale compared to a previous assessment. Nevertheless, for our understanding of the complex relationship between biological and psychosocial risk factors and PSD and PSA, our findings provide relevant indications.

As we studied a broad range of imaging markers in association with PSD and PSA (Chapter 6), we chose to look at the development of PSD and PSA at any time point in the one-year follow-up period, since the sample size was too small to look at interactions with time. In this study, we used stricter criteria for PSD, by defining it as a diagnosis of either major depressive disorder or minor depression on the M.I.N.I. on at least one assessment during the one-year follow-up period, to ensure that the depression was clinically relevant. 


\section{METHODOLOGICAL CONSIDERATIONS}

The most important strengths and limitations of the studies in this thesis will be discussed below.

\section{Strengths and limitations of the systematic review and meta-analysis}

A systematic review and meta-analysis (Chapter 2) was conducted to provide a comprehensive and up-to-date evaluation of imaging markers associated with PSD and PSA. A large amount of PSD studies was included ( $n=132$ ), which made it possible to conduct meta-analyses on lesion laterality, lesion type, and also on lesion location associated with PSD. In addition, an extensive qualitative overview was provided of a broad range of imaging markers associated with PSD and PSA. The systematic review and meta-analysis (Chapter 2) also had limitations. The most important limitation was the small amount of studies on PSA, which compromised the generalizability of the results. Moreover, the heterogeneity between PSA studies was small, so the results need to be interpreted with caution. Nevertheless, we believe they are still of importance, but also emphasize the need for more PSA studies to be able to draw firm conclusions. Furthermore, moderate to high (unexplained) heterogeneity in some meta-analyses indicated large differences in methodology between studies. Meta-regression analyses were performed to identify potential sources of heterogeneity, but only study phase for PSD studies and imaging method for PSA studies could be identified. Probably also other potential variables (e.g. years of education, cognitive status) that were not included as covariates in the meta-regression analyses might explain some of the heterogeneity, but due to the large variability in methods and availability of data between studies we were not able to examine this.

\section{Strengths and limitations of the CASPER study}

Strengths of the CASPER study (Chapter 4 - 8) include the direct comparison of associated risk factors of PSD and PSA in the same cohort. Furthermore, the longitudinal design enabled us to examine differences in trajectories between patients with and without PSD or PSA in a relatively large sample. Also, the design allowed us to study temporal relationships between the development of different syndromes. The dropout of participants was rather low at follow-up, resulting in 213 (85\%) study completers at six-month follow-up and 219 study completers (88\%) at 12-month follow-up. Another strength was the extensive neuropsychological assessment that was conducted at all time points, which covered a screening instrument to assess global cognition, and several test instruments to measure (verbal) episodic memory, working memory, information processing speed, and executive function. Also, a number of validated instruments (e.g. AES, ${ }^{26}$ MADRS, ${ }^{29}$ 
M.I.N.I. ${ }^{30}$ ) was administered to evaluate presence and severity of PSD and PSA at all time points. This is one of the few studies that studied factors associated with both PSD or PSA in a prospective cohort, as the majority of studies focused only on one of the two, or only on cross-sectional associations. Another strength is that analyses with PSD as outcome were corrected for PSA and vice versa. This was done to be able to study the effect of a certain factor on PSD independently of PSA and vice versa. Lastly, the majority received a 3-Tesla MRI at baseline (three months post-stroke), which provided a range of imaging data, consisting of advanced volumetric and visually rated imaging markers.

The CASPER study had the following limitations. First, the exclusion of patients with severe aphasia, pre-existing cognitive impairment, and other neurological conditions that are known to affect cognition may limit the generalizability of our results to the general population of stroke survivors. However, patients with severe aphasia had to be excluded as participants needed to understand the study procedure fully to be able to give informed consent. Also, patients with preexisting cognitive impairment were excluded to ensure that cognitive impairment was due to vascular pathology, and not due to other origins. Second, the number of patients with PSA was rather small in the CASPER cohort. In literature, there is no consensus on how to define PSA, although many previous stroke studies defined PSA according to the same criteria as we did. ${ }^{37-39}$ A possible explanation for the low frequency could be that our cohort included largely mild to moderately severe stroke, as participation was too burdensome for a large group of eligible patients (60\%). Patients who refused participation were older and more frequently female compared with patients who agreed to participate. Thus, these factors could have contributed to a selection bias in our cohort. Therefore, all analyses were at least corrected for age, sex, and level of education. Third, not all patients included at baseline managed to complete all follow-up assessments, which resulted in missing data. However, the frequency of PSD and PSA did not differ between patients who dropped-out at one or two time points compared with patients who completed all measurements. By applying random-effects linear mixed models or generalized estimating equation (GEE) logistic models we accounted for missing data, as these methods can also model trajectories over time for participants who did not complete all measurements and does not require imputation of missing data. Fourth, patients were followed in the first 15-months post-stroke, which is generally assumed to be the period of most recovery. Although this mediumterm follow-up provides the opportunity to look at differences in trajectories over time, an even longer follow-up period (2-5 years) would allow to also examine associations between PSD and PSA and other outcomes, like dementia and mortality, as PSD has been associated with an increased risk for mortality, ${ }^{40,41}$ and 
this has not been examined in PSA yet. Lastly, imaging data were only collected at baseline as due to logistical and financial reasons it was not possible to repeat MRI at follow-up. However, these data would have been interesting, as changes in MRI markers could then have been related to changes in PSD or PSA as well.

\section{CONCEPTUAL AND CLINICAL IMPLICATIONS}

The present findings provide evidence that, at least in the stroke population, different risk factors are associated with apathy and depression. However, this does not necessarily mean that they are strictly different entities, as apathy can also be a symptom of depression, which complicates the conceptualization of these constructs. With respect to symptoms, a depressive disorder can differ greatly between individuals, with for example some patients particularly feeling sadness and lowered mood, while in others anhedonia (i.e. lack of feeling pleasure and loss of interest) dominates. The latter greatly overlaps with the diagnostic criteria for apathy. ${ }^{42}$ This means that in some depressed patients, there is much overlap with apathy symptoms, whereas in others this overlap may be minimal or absent. Also, some patients may experience apathy in absence of depressed mood. With respect to the differentiation between syndrome and symptom, this indicates that apathy can be a symptom of depression, but can also develop as an independent syndrome in absence of depression. To distinguish apathy from depression, it is suggested that apathy is more likely a behavioral disorder, characterized by lack of primary motivation and affective blunting, ${ }^{43}$ while depression can be defined as a disorder of mood. ${ }^{44}$ Particularly the absence of negative moods (i.e. sadness, feelings of guilt, helplessness, hopelessness) can help to distinguish apathy from depression. ${ }^{44}$ However, a depressive disorder can also be defined in absence of negative mood, if the core symptom anhedonia is present. Therefore, instead of following a categorical approach, which classifies disorders on the basis of fulfilling symptoms and characteristics that are typical of the disorder, it may be better to conceptualize apathy and depression following a dimensional or continuous approach. ${ }^{45}$ This has the advantage that it overcomes the problem of applying diagnostic criteria or (arbitrary) cut-offs to define clinically relevant depression or apathy. Also, in statistical analyses, continuous measures result in greater statistical power. Thus, the findings presented in this thesis should also be interpreted from a dimensional perspective, meaning that differential risk factors are associated with increased levels of apathetic and depressive symptoms after stroke.

Nevertheless, an important difficulty remains with respect to the measurement of depression and apathy, since no objective measurement instrument is available. Therefore, assessments are always dependent on the way patients respond to 
questions asked by the clinician and the clinicians' interpretation of this. Since particularly patients with apathy show lack of insight ${ }^{44}$ and may not be able to provide a good picture of how they are feeling, this hampers the reliability of evaluating the presence of mood disorders in this patient group. Apathy is characterized by a reduction in motivationally driven behavior and poor reward sensitivity. Therefore, apathy can also be measured with a behavioural task, like a reward sensitivity task. Studies using this task showed that stroke patients ${ }^{46}$ and patients with Parkinson's disease ${ }^{47}$ with apathy showed impaired reward sensitivity. This reward insensitivity appeared to be related to reduced pupillary dilation, ${ }^{47}$ and may therefore provide a novel and useful objective clinical marker of apathy.

The indications for differences in risk factors involved in the development of symptoms of PSD and PSA that are described in this thesis are helpful to denote patients particularly vulnerable for developing these neuropsychiatric disorders. It supports the idea that there are important differences between them and contributes to the understanding of their pathophysiology and showed that both biological and psychosocial factors play a role. So far, little knowledge was available with respect to risk factors of PSA. Therefore, our findings are particularly important for the comprehension of the underlying etiology of PSA, which seems to be different, in some extent, from PSD. Thus, the studies described in this thesis provided more insight into the differential role of biopsychosocial factors on the course of PSD and PSA. However, more research is needed to understand the whole picture as not all potentially involved factors could have been studied here.

Our findings indicate that PSD and PSA are prevalent after stroke, with almost 45\% of the patients showing PSD, PSA, or a combination of the two in the post-acute stroke phase. This has important clinical implications, since both PSD and PSA are associated with worse clinical outcome..$^{2,21}$ Therefore, it is important that clinicians are aware of both syndromes and that they are recognized correctly. Also, they should be taken into consideration during rehabilitation, as PSD and PSA are known to have a negative impact on functional recovery, ${ }^{48}$ and are associated with poor involvement in rehabilitation. ${ }^{49}$

The findings in this thesis provide evidence for the suggestion that PSD and PSA are independent syndromes, with differences in symptomatology, risk factors, and course. Health professionals should be aware that PSA can develop independently from PSD. Therefore screening for PSA is important, and a standardized protocol for health professionals is recommended. Apathy symptoms are usually noticed by caregivers or close relatives of the patients, but are often not reported as complaints by the patients themselves, as they may not feel affected by these symptoms. Even though apathetic symptoms may be observed by caregivers or close relatives, they are usually not aware that these are somehow associated 
with the stroke and are mainly bothered or irritated by the passiveness and lack of interest of the patient. Therefore, also special attention should be given to caregivers, as living with someone with apathy can be particularly burdensome, ${ }^{50}$ and they are often not capable to deal with it. Psychoeducation programs for caregivers and stroke survivors focusing on awareness of PSD and PSA and on how to cope with these syndromes would therefore be helpful.

In some patients, neuropsychiatric complaints develop only after a long period of time, in the chronic stroke phase, in which the patients usually no longer have regular check-ups in the hospital or rehabilitation center. ${ }^{51}$ This implicates that the general practitioner (GP) should be aware of this. PSD in the chronic stroke phase has been shown to be related to worse outcome with respect to instrumental activities of daily living and quality of life. ${ }^{52}$ Furthermore, one study showed that depressive symptoms were found to be a risk factor for the presence of unmet demands three years after stroke, with a third of stroke patients not receiving the appropriate care..$^{3}$ In a prospective cohort study with five year follow-up, PSA became more prevalent with time, and risk factors were dementia, functional impairment, high levels of depression, and cerebrovascular events. ${ }^{6}$ The presence of PSD and PSA in the post-acute and chronic stroke phase has a negative impact on clinical outcome. Therefore, it is important that there is sufficient attention for these conditions, also at a later time point post-stroke. Hence, it is recommended that GPs are attentive to neuropsychiatric symptoms and cognitive impairment in stroke patients. Also attention should be paid to patients who do not report any complaints, because stroke can affect disease insight, also called anosognosia. ${ }^{54}$ In a study by Aben and colleagues, ${ }^{55}$ GPs recognized PSD in less than $40 \%$ of the depressed patients, which stresses that education with respect to neuropsychiatric symptoms after stroke may be helpful for GPs, but undoubtedly also for other clinicians working in the field of stroke care. The current development of specialized stroke after-care clinics, in which there is specific expertise with respect to lateonset and 'invisible' complaints after stroke, like neuropsychiatric and cognitive complaints, is a good and promising development in this sense.

Taken together, there should be more awareness for the possible development of PSD and PSA after stroke in health care, so that these syndromes can be detected in time. Special attention should be paid to correct recognition of PSA, which could be reached by implicating standardized diagnostic criteria or standard use of a screening instrument like the AES, which can be applied by health care professionals. Regular visits to a GP in the first years post-stroke would be helpful for early recognition of symptoms, which may result in more adequate treatment or prevention of developing neuropsychiatric symptoms. Also, psychoeducation for stroke survivors and their caregivers with respect to neuropsychiatric symptoms 
after stroke would be recommended, to increase awareness that in addition to physical complaints, speech difficulties, and other 'more visible' consequences of stroke, also neuropsychiatric symptoms may develop. Correct recognition of these syndromes will result in better understanding and also better handling of these symptoms, but it is the question whether this will also help in finding treatment possibilities. It is plausible that early identification is beneficial for clinical prognosis, if there are appropriate treatment strategies available. However, this was not examined in the present thesis and remains an important topic for future research.

\section{RECOMMENDATIONS FOR FUTURE RESEARCH}

Previous research on neuropsychiatric symptoms after stroke focused mainly on the prevalence and biological and psychosocial factors associated with PSD. Over the last fifteen years there has been an increase in attention for PSA. However, only few longitudinal studies are available that looked at differences in associated risk factors of PSD and PSA in the same stroke cohort. Therefore, the present thesis provided important insight into the differential factors that play a role in the development of PSD and PSA. Based on our findings, some recommendations can be given for future research.

First of all, the lack of a consensus with respect to instruments and cut-offs for assessing PSD and PSA is a major difficulty, as it leads to under-recognition of these syndromes and large heterogeneity between studies. Consensus on the application of rating scales and cut-offs in future studies would be helpful to overcome this, which makes it easier to assemble and compare study findings. The results of this thesis indicate that different factors play a role in the development of PSD and PSA. Though a broad range of factors was covered, this only comprised a small part of factors that are potentially involved. Therefore, additional large cohort studies focusing on both syndromes that cover a broad range of both biological and psychosocial factors are needed to provide more insight into the complex multifactorial origins of PSD and PSA. For example, the role of inflammatory cytokines that are released after ischemia, and genetic polymorphisms that could predispose stroke patients to develop neuropsychiatric symptoms are studied to a much lesser extent. In CASPER, data on several inflammatory markers and epigenetics are available and will be investigated. So, research on biological determinants of neuropsychiatric symptoms needs to incorporate all these potential factors, and study their interrelationships and interactions with psychosocial factors, to be able to shed light on the complex multifactorial origin of PSD and PSA. The study of multiple predictors to find subtle associations with PSD and PSA requires large sample sizes to reach sufficient power. Therefore, CASPER was designed to allow harmonization with other (comparable) stroke cohorts. This harmonization provides the opportunity to 
conduct large individual patient data meta-analyses, which have several advantages over standard meta-analyses that only use published results. ${ }^{56}$ Therefore, CASPER joined the STROKOG consortium (https://cheba.unsw.edu.au/group/strokog), ${ }^{57}$ which has built a database with more than 12,000 stroke patients already and will likely yield important results concerning factors associated with PSD and PSA. This will hopefully result in potential targets for treatment and prevention of these syndromes, which is another urgent area for future research.

More research into optimal pharmacological and psychological treatment for PSD is necessary. There are currently no guidelines with respect to effective approaches, $^{58}$ and a large amount of patients remains currently untreated or inadequately treated..$^{58} \mathrm{~A}$ meta-analysis on treatment for PSD found a moderate effect of antidepressants, whereas no effect was found for psychotherapy. ${ }^{59}$ Both European ${ }^{60}$ and American ${ }^{61}$ guidelines recommend using antidepressants as pharmaceutical treatment for PSD, although they require that effectiveness should be clearly monitored. However, there is debate around the safety of prescribing selective serotonin reuptake inhibitor (SSRI) antidepressants in stroke patients, as they appeared to be associated with increased risk of mortality. ${ }^{41}$ This is probably related to an increased risk for hemorrhage ${ }^{62}$ due to an interaction with anticoagulants (blood thinners). ${ }^{63}$ Therefore, further investigation into successful treatment options is clearly needed. Also, routinely screening for PSD in the first year(s) post-stroke would be helpful to treat and prevent PSD adequately, and more research is needed to examine whether this also has a beneficial effect on clinical prognosis in these patients.

For treatment of apathy there are currently no recommended treatment options. ${ }^{8}$ There is insufficient evidence showing the effectiveness of pharmacological treatments, ${ }^{64,65}$ and most research on apathy treatment has been performed in other disease populations, like Alzheimer's ${ }^{66}$ and Parkinson's Disease. ${ }^{67}$ The use of SSRIs may have a negative influence on apathy, ${ }^{68}$ therefore it is particularly important that apathy is distinguished from depression, and that alternative treatment options will be available for depressed patients with co-morbid apathy. Hence, large randomized controlled trials are needed to investigate potential treatment targets for PSA. The results of the CASPER study described in this thesis provided important information on which patients may be vulnerable to develop apathy after stroke (i.e. cognitive impairment, low extraversion, global brain atrophy and high cSVD burden). These findings are important for a better understanding of the biological and psychosocial processes involved in the development of this syndrome, and how this differs from the underlying factors involved in PSD. Eventually, this information will be helpful in finding potential treatment targets for PSA. 


\section{Chapter 9}

\section{CONCLUSIONS}

The findings presented in this thesis provide evidence that different factors are involved in the development of PSD and PSA, which are of biological and psychosocial origin. This improves our understanding of the pathophysiology of these entities and supports the idea that PSA can occur as an independent syndrome, in absence of PSD. Information on cognitive functioning, level of fatigue, (premorbid) personality traits, and degenerative and vascular brain pathology should be considered by health professionals. Differences in these vulnerability factors between patients are important to incorporate, by means of providing patient-tailored treatment. Also, during the acute stroke phase, clinicians should be aware of patients with high vulnerability to develop PSD and PSA, and patients and their caregivers should be informed that these neuropsychiatric symptoms can develop, also at a later post-stroke stage. Earlier detection of these conditions, and better distinction between PSD and PSA treatment may hopefully lead to development of treatment strategies and a better clinical prognosis after stroke. 


\section{REFERENCES}

1. Hackett ML, Pickles K. Part i: Frequency of depression after stroke: An updated systematic review and meta-analysis of observational studies. Int J Stroke. 2014;9:1017-1025.

2. van Dalen JW, van Charante EPM, Nederkoorn PJ, van Gool WA, Richard E. Poststroke apathy. Stroke. 2013;44:851-860.

3. Yang S, Hua P, Shang X, Cui Z, Zhong S, Gong G, et al. A significant risk factor for poststroke depression: The depression-related subnetwork. J Psychiatry Neurosci. 2015;40:259.

4. Yang S, Hua P, Shang X, Cui Z, Zhong S, Gong G, et al. Deficiency of brain structural sub-network underlying post-ischaemic stroke apathy. Eur J Neurol. 2015;22:341-347.

5. Wu S, Barugh A, Macleod M, Mead G. Psychological associations of poststroke fatigue. Stroke. 2014;45:1778-1783

6. Brodaty H, Liu Z, Withall A, Sachdev PS. The longitudinal course of post-stroke apathy over five years. J Neuropsychiatry Clin Neurosci. 2013;25:283-291.

7. Withall A, Brodaty H, Altendorf A, Sachdev PS. A longitudinal study examining the independence of apathy and depression after stroke: The sydney stroke study. Int Psychogeriatr. 2011;23:264273.

8. Hackett ML, Köhler S, T O'Brien J, Mead GE. Neuropsychiatric outcomes of stroke. Lancet Neurol. 2014;13:525-534.

9. Wu S, Mead G, Macleod M, Chalder T. Model of understanding fatigue after stroke. Stroke. 2015;46:893-898.

10. Acciarresi M, Bogousslavsky J, Paciaroni M. Post-stroke fatigue: Epidemiology, clinical characteristics and treatment. Eur Neurol. 2014;72:255-261.

11. Kendler KS, Gatz M, Gardner CO, Pedersen NL. Personality and major depression: A Swedish longitudinal, population-based twin study. Arch Gen Psychiatry. 2006;63:1113-1120.

12. Noteboom A, Beekman AT, Vogelzangs N, Penninx BW. Personality and social support as predictors of first and recurrent episodes of depression. J Affect Disord. 2016;190:156-161.

13. Hwang SI, Choi KI, Park OT, Park S-W, Choi ES, Yi S-H. Correlations between pre-morbid personality and depression scales in stroke patients. Ann Rehabil Med. 2011;35:328-336.

14. Aben I, Denollet J, Lousberg R, Verhey F, Wojciechowski F, Honig A. Personality and vulnerability to depression in stroke patients. Stroke. 2002;33:2391-2395.

15. Yoon KL, Maltby J, Joormann J. A pathway from neuroticism to depression: Examining the role of emotion regulation. Anxiety Stress Coping. 2013;26:558-572.

16. Wongpakaran N, Wongpakaran T, van Reekum R. Social inhibition as a mediator of neuroticism and depression in the elderly. BMC Geriatr. 2012;12:1-7.

17. Van Mierlo ML, Van Heugten CM, Post MW, De Kort PL, Visser-Meily JM. Psychological factors determine depressive symptomatology after stroke. Arch Phys Med Rehabil. 2015;96:1064-1070.

18. Douiri A, Rudd AG, Wolfe CD. Prevalence of poststroke cognitive impairment. Stroke. 2013;44:138145.

19. Snowdon DA, Greiner LH, Mortimer JA, Riley KP, Greiner PA, Markesbery WR. Brain infarction and the clinical expression of alzheimer disease: The nun study. JAMA. 1997;277:813-817.

20. Sachdev P, Kalaria R, O'Brien J, Skoog I, Alladi S, Black SE, et al. Diagnostic criteria for vascular cognitive disorders: A vascog statement. Alzheimer Dis Assoc Disord. 2014;28:206-218.

21. Ayerbe L, Ayis $S$, Wolfe CD, Rudd AG. Natural history, predictors and outcomes of depression after stroke: Systematic review and meta-analysis. Br J Psychiatry. 2013;202:14-21.

22. Brodaty $H$, Withall $A$, Altendorf A, Sachdev PS. Rates of depression at 3 and 15 months poststroke and their relationship with cognitive decline: The Sydney stroke study. Am J Geriatr Psychiatry. 2007; 15:477-486.

23. Mayo NE, Fellows LK, Scott SC, Cameron J, Wood-Dauphinee S. A longitudinal view of apathy and its impact after stroke. Stroke. 2009;40:3299-3307. 
24. Whyte EM, Mulsant BH. Post stroke depression: Epidemiology, pathophysiology, and biological treatment. Biol Psychiatry. 2002;52:253-264.

25. De Ryck A, Fransen E, Brouns R, Geurden M, Peij D, Mariën P, et al. Poststroke depression and its multifactorial nature: Results from a prospective longitudinal study. J Neurol Sci. 2014;347:159166.

26. Marin RS, Biedrzycki RC, Firinciogullari S. Reliability and validity of the Apathy Evaluation Scale. Psychiatry Res. 1991;38:143-162.

27. Clarke D, Ko J, Kuhl E, van Reekum R, Salvador R, Marin R. Are the available apathy measures reliable and valid? A review of the psychometric evidence. J Psychosom Res. 2011;70:73-97.

28. Clarke DE, Reekum Rv, Simard M, Streiner DL, Freedman M, Conn D. Apathy in dementia: An examination of the psychometric properties of the apathy evaluation scale. J Neuropsychiatry Clin Neurosci. 2007;19:57-64.

29. Montgomery SA, Asberg M. A new depression scale designed to be sensitive to change. $\mathrm{Br} \mathrm{J}$ Psychiatry. 1979;134:382-389.

30. Overbeek I, Schruers K, Griez E. Mini international neuropsychiatric interview: Nederlandse versie 5.0. 0. DSM-IV [Dutch version]. Maastricht, The Netherlands: Universiteit Maastricht. 1999.

31. Kang H-J, Stewart R, Kim J-M, Jang J-E, Kim S-Y, Bae K-Y, et al. Comparative validity of depression assessment scales for screening poststroke depression. J Affective Disord. 2013;147:186-191.

32. Sheehan D, Janavs J, Harnett-Sheehan K, Sheehan M, Gray C, Lecrubier Y, et al. Mini international neuropsychiatric interview, version 6.0. Tampa, USA: University of South Florida College of Medicine, Paris, France: Centre Hospitalier Sainte-Anne. 2009.

33. Spitzer RL, Williams JB, Gibbon M, First MB. User's guide for the structured clinical interview for DSM-III-R: SCID. 1990.

34. Zigmond AS, Snaith RP. The hospital anxiety and depression scale. Acta Psychiatr Scand. 1983;67:361-370.

35. Beck AT, Ward CH, Mendelson M, Mock J, ERBAUGH J. An inventory for measuring depression. Arch Gen Psychiatry. 1961;4:561-571.

36. Yesavage JA, Brink TL, Rose TL, Lum O, Huang V, Adey M, et al. Development and validation of a geriatric depression screening scale: A preliminary report. J Psychiatr Res. 1983;17:37-49.

37. Brodaty H, Sachdev PS, Withall A, Altendorf A, Valenzuela MJ, Lorentz L. Frequency and clinical, neuropsychological and neuroimaging correlates of apathy following stroke-the Sydney stroke study. Psychol Med. 2005;35:1707-1716.

38. Caeiro L, Ferro JM, Pinho e Melo T, Canhão P, Figueira ML. Post-stroke apathy: An exploratory longitudinal study. Cerebrovasc Dis. 2013;35:507-513.

39. Tang W, Chen Y, Liang H, Chu WC, Mok V, Ungvari GS, et al. Location of infarcts and apathy in ischemic stroke. Cerebrovasc Dis. 2013;35:566-571.

40. Jørgensen TS, Wium-Andersen IK, Wium-Andersen MK, Jørgensen MB, Prescott E, Maartensson $\mathrm{S}$, et al. Incidence of depression after stroke, and associated risk factors and mortality outcomes, in a large cohort of danish patients. JAMA Psychiatry. 2016;73:1032-1040.

41. Ayerbe L, Ayis S, Crichton SL, Rudd AG, Wolfe CD. Explanatory factors for the increased mortality of stroke patients with depression. Neurology. 2014;83:2007-2012.

42. Starkstein SE, Leentjens AF. The nosological position of apathy in clinical practice. J Neurol Neurosurg Psychiatry. 2008;79:1088-1092.

43. Kirsch-Darrow L, Marsiske M, Okun MS, Bauer R, Bowers D. Apathy and depression: Separate factors in Parkinson's disease. J Int Neuropsychol Soc. 2011;17:1058-1066.

44. Tagariello P, Girardi P, Amore M. Depression and apathy in dementia: Same syndrome or different constructs? A critical review. Arch Gerontol Geriatr. 2009;49:246-249.

45. Kraemer HC, Noda A, O'Hara R. Categorical versus dimensional approaches to diagnosis: Methodological challenges. J Psychiatr Res. 2004;38:17-25. 
46. Rochat L, Van der Linden M, Renaud O, Epiney J-B, Michel P, Sztajzel R, et al. Poor reward sensitivity and apathy after stroke implication of basal ganglia. Neurology. 2013;81:1674-1680.

47. Muhammed K, Manohar S, Yehuda MB, Chong TT-J, Tofaris G, Lennox G, et al. Reward sensitivity deficits modulated by dopamine are associated with apathy in parkinson's disease. Brain. 2016;139:2706.

48. Hama S, Yamashita H, Yamawaki S, Kurisu K. Post-stroke depression and apathy: Interactions between functional recovery, lesion location, and emotional response. Psychogeriatrics. 2011;11:68-76.

49. Turner-Stokes L, Hassan N. Depression after stroke: A review of the evidence base to inform the development of an integrated care pathway. Part 1: Diagnosis, frequency and impact. Clin Rehabil. 2002;16:231-247.

50. de Vugt ME, Riedijk SR, Aalten P, Tibben A, van Swieten JC, Verhey FR. Impact of behavioural problems on spousal caregivers: A comparison between alzheimer's disease and frontotemporal dementia. Dement Geriatr Cogn Disord. 2006;22:35-41.

51. Visser-Meily J, Van Den Bos G, Kappelle L. Better acute treatment induces more investments in chronic care for stroke patients. Int J Stroke. 2009;4:352-353.

52. van de Port IG, Kwakkel G, Bruin M, Lindeman E. Determinants of depression in chronic stroke: A prospective cohort study. Disabil Rehabil. 2007;29:353-358.

53. van de Port IG, van den Bos GA, Voorendt M, Kwakkel G, Lindeman E. Identification of risk factors related to perceived unmet demands in patients with chronic stroke. Disabil Rehabil. 2007;29:1841-1846.

54. Starkstein SE, Jorge RE, Robinson RG. The frequency, clinical correlates, and mechanism of anosognosia after stroke. Can J Psychiatry. 2010;55:355-361.

55. Aben I, Verhey F, Beusmans G, Lodder J. Depressie na een cva: Signalering, diagnostiek en behandeling in de huisartsenpraktijk. Huisarts Wet. 2003;46:373-379.

56. Clarke MJ. Individual patient data meta-analyses. Best Pract Res Clin Obstet Gynaecology. 2005;19:47-55.

57. Sachdev PS, Lo JW, Crawford JD, Mellon L, Hickey A, Williams D, et al. Strokog (stroke and cognition consortium): An international consortium to examine the epidemiology, diagnosis, and treatment of neurocognitive disorders in relation to cerebrovascular disease. Alzheimers Dement 2017;7:11-23.

58. Paolucci S. Advances in antidepressants for treating post-stroke depression. Expert Opin Pharmacother. 2017;18:1011-1017.

59. Hackett ML, Anderson CS, House AO, Xia J. Interventions for treating depression after stroke. Stroke. 2009;40:487-488.

60. Quinn TJ, Paolucci S, Sunnerhagen KS, Sivenius J, Walker MF, Toni D, et al. Evidence-based stroke rehabilitation: An expanded guidance document from the European Stroke Organisation (ESO) guidelines for management of ischaemic stroke and transient ischaemic attack 2008. J Rehabil Med. 2009;41:99-111.

61. Miller EL, Murray L, Richards L, Zorowitz RD, Bakas T, Clark P, et al. Comprehensive overview of nursing and interdisciplinary rehabilitation care of the stroke patient. Stroke. 2010;41:2402-2448.

62. Mortensen JK, Larsson H, Johnsen SP, Andersen G. Post stroke use of selective serotonin reuptake inhibitors and clinical outcome among patients with ischemic stroke. Stroke. 2013;44:420-426.

63. Renoux C, Vahey S, Dell'Aniello S, Boivin J-F. Association of selective serotonin reuptake inhibitors with the risk for spontaneous intracranial hemorrhage. JAMA Neurol. 2017;74:173-180.

64. Starkstein SE, Brockman S, Hatch KK, Bruce DG, Almeida OP, Davis WA, et al. A randomized, placebo-controlled, double-blind efficacy study of nefiracetam to treat poststroke apathy. $J$ Stroke Cerebrovasc Dis. 2016;25:1119-1127.

65. Jorge RE, Starkstein SE, Robinson RG. Apathy following stroke. Can J Psychiatry. 2010;55:350354. 
66. Theleritis C, Siarkos K, Katirtzoglou E, Politis A. Pharmacological and nonpharmacological treatment for apathy in alzheimer disease: A systematic review across modalities. J Geriatr Psychiatry Neurol. 2017;30:26-49.

67. Pagonabarraga J, Kulisevsky J, Strafella AP, Krack P. Apathy in parkinson's disease: Clinical features, neural substrates, diagnosis, and treatment. Lancet Neurol. 2015;14:518-531.

68. Wongpakaran N, van Reekum R, Wongpakaran T, Clarke D. Selective serotonin reuptake inhibitor use associates with apathy among depressed elderly: A case-control study. Ann Gen Psychiatry. $2007 ; 6: 1-6$. 
General discussion 
ADDENDUM 
SUMMARY

NEDERLANDSE SAMENVATTING (DUTCH SUMMARY)

KNOWLEDGE VALORIZATION

DANKWOORD (ACKNOWLEDGEMENTS)

THESIS DEFENSES FROM MHENS

(School for Mental Health and Neuroscience)

LIST OF PUBLICATIONS

CURRICULUM VITAE 
Addendum 


\section{SUMMARY}

Post-stroke depression (PSD) and apathy (PSA) are two of the most common neuropsychiatric outcomes after stroke, and have substantial overlap in symptoms. Both are associated with poor prognosis, as they have a negative influence on functional recovery and quality of life after stroke. Despite a significant overlap in associated factors, several studies found indications for differences in associated risk factors between PSD and PSA, suggesting that these might represent independent syndromes. However, findings were largely based on cross-sectional data and studies on PSA are relatively scarce. Furthermore, only few studies have looked at the development, course, and associated factors of both syndromes at the same time in a longitudinal study. In this thesis, we aimed to examine the influence of biological and psychosocial risk factors on the development and course of PSD and PSA, and whether differences could be found between the associations with these two syndromes.

In Chapter 2 we provided a systematic review and meta-analysis on imaging markers associated with PSD and PSA. We examined the association between PSD or PSA and stroke lesion laterality, type, and location, also stratified by study phase (i.e. acute, post-acute, chronic phase after stroke) in a meta-analysis. Associations with other imaging markers (e.g. white matter hyperintensities (WMH), atrophy, lesion volume, number of lesions, cerebral microbleeds) were reviewed qualitatively to provide an up-to-date overview. In the post-acute stroke phase (0.5 - 6 months post-stroke), PSD was associated with frontal lesions and lesions in the basal ganglia. No associations were found with lesion laterality (i.e. left or right hemisphere) or lesion type (i.e. ischemic or hemorrhagic stroke). PSA was more frequent after hemorrhagic stroke in the acute stroke phase ( $<15$ days), whereas it was more frequent after ischemic stroke in the post-acute phase. Furthermore, PSA did not depend on lesion laterality or location, but the amount of available PSA studies was limited. The present findings suggest that lesion location rather than lesion laterality or type is an important risk factor for PSD in the post-acute stroke phase. In contrast, lesion type rather than location or laterality might be an important risk factor for PSA, though additional PSA studies are needed to confirm this.

In Chapter 3 the rationale and design of The Cognition and Affect after Stroke: a Prospective Evaluation of Risks (CASPER) study is presented. The CASPER study is a one-year prospective clinical stroke cohort follow-up study, which was performed to study predictors of vascular cognitive impairment, depression and apathy after stroke. For this study, 250 patients who suffered from ischemic or hemorrhagic stroke were included. A baseline measurement was scheduled at three months 
post stroke and consisted of a venepuncture, recording of socio-demographic information, a neuropsychological assessment and neuropsychiatric interview, and a structural 3-Tesla Magnetic Resonance Imaging (MRI) of the brain. The neuropsychological assessment and neuropsychiatric interview was repeated at 6-month and 12-month follow-up.

In Chapter 4 we studied the temporal relationships between post-stroke fatigue (PSF) and PSD and between PSF and PSA. The effect of PSF at three months poststroke on the course of depressive and apathetic symptoms over a period of 12-month follow-up was measured. In addition, we studied the reverse direction by examining the effect of PSD (or PSA) at three months post-stroke on the course of fatigue symptoms. The results showed a bi-directional association between PSF and PSD, whereas PSA was not associated with PSF. Levels of PSF and PSD remained stable over time. Therefore, a timely detection of PSF and PSD conditions in stroke patients is important, as they tend to persist.

In Chapter 5 we examined the role of premorbid personality traits in the development and course of symptoms of depression and apathy after stroke. We found an association between high neuroticism and high levels of depression at baseline (three months post-stroke), and this figure remained stable during the 12-month follow-up. In contrast, low extraversion was associated with high levels of apathy at baseline, and this association remained stable over time as well. These findings show the importance of taking personality traits into account as a potential vulnerability factor for PSD and PSA.

In Chapter 6 we studied the association between lesion-related imaging markers and background markers of vascular and degenerative pathology and the development of PSD and PSA. The results showed that global brain atrophy and severe cerebral small vessel disease (cSVD) burden, but not stroke lesion-related markers, were associated with the development of PSA in the first year post-stroke, independent of co-morbid PSD and stroke lesion volume. Global brain atrophy and medium CSVD burden were associated with the development of PSD, after correction for stroke lesion volume and history of depression. After correction for co-morbid PSA, only medium cSVD remained significantly associated with PSA. This suggests that atrophy and increased cSVD burden are important predictors for the development of PSA, and, less strongly, for PSD.

In Chapter 7 we performed longitudinal analyses to examine the influence of "any Vascular Cognitive Impairment (VCl)" (impairment in at least one cognitive domain due to vascular brain pathology) and degree of $\mathrm{VCl}$ (impairment in multiple cognitive domains versus impairment in a single domain) on the course of depression and apathy symptoms over time. Any $\mathrm{VCl}$ predicted an increase in 
apathy levels from three to 15 months post-stroke, which was most pronounced in patients with $\mathrm{VCl}$ in multiple cognitive domains. Domain-specific analyses showed that particularly impairment in executive dysfunction and slowed information processing speed was associated with an increase in apathy symptoms over time. None of the cognitive variables predicted the course of depressive symptoms. These results implicate that although apathy symptoms may be absent in the first months after stroke, these symptoms can develop over time in patients with $\mathrm{VCl}$.

In Chapter $\mathbf{8}$ we examined whether executive dysfunctions (ED) denote a distinct PSD subtype by studying whether the co-occurrence of ED and PSD in a depressionexecutive dysfunction depression subtype (DES) is associated with different clinical and neuroimaging correlates and a worse prognosis. The DES-subtype was associated with higher WMH volume, a higher frequency of old infarcts, more global brain atrophy, and higher total cSVD burden on MRI at three months poststroke. Longitudinal analyses showed that DES was associated with a less benign course, including a non-improving pattern of worse cognitive performance compared with ED-only and chronic high levels of depression compared with PSD-only. Our findings suggest that DES is associated with increased generalized (vascular) brain pathology, which provides support for a strong biological origin of depression in the DES group compared with the PSD-only group.

Chapter 9 provided a general discussion of the findings presented in this thesis. Also, methodological considerations and conceptual and clinical implications were addressed and recommendations for future research were discussed. Furthermore, this last chapter includes a summary of the findings, a knowledge valorisation, acknowledgements, a list of publications, and information about the author.

Overall, the studies in this thesis provide evidence that different factors are involved in the development of PSD and PSA, which are of biological and psychosocial origin. This improves our understanding of the pathophysiology of these entities and supports the hypothesis that PSA can occur as an independent syndrome, in absence of PSD. Differences in vulnerability factors between patients are important to incorporate, by means of providing patient-tailored treatment. Earlier detection of these conditions, and better distinction between PSD and PSA may hopefully lead to development of treatment strategies and a better clinical prognosis after stroke. 
Addendum 


\section{NEDERLANDSE SAMENVATTING}

Depressie na een beroerte (oftewel post-stroke-depressie, PSD) en post-stroke apathie (PSA) zijn twee van de vaakst voorkomende neuropsychiatrische syndromen na een beroerte, en er is een substantiële overlap in symptomen tussen deze syndromen. Beide zijn geassocieerd met een slechte prognose, omdat ze een negatieve invloed hebben op functioneel herstel en kwaliteit van leven na een beroerte. Ondanks een significante overlap in geassocieerde factoren hebben verschillende studies aanwijzingen gevonden voor verschillen in geassocieerde risicofactoren tussen PSD en PSA, wat suggereert dat ze wellicht onafhankelijke syndromen representeren. Echter, bevindingen zijn grotendeels gebaseerd op cross-sectionele data en studies naar PSA zijn relatief schaars. Bovendien, hebben slechts enkele studies gekeken naar de ontwikkeling, het beloop, en geassocieerde factoren van beide syndromen tegelijkertijd binnen een longitudinale studie. Het doel van dit proefschrift was om de invloed te onderzoeken van biologische en psychosociale risicofactoren op de ontwikkeling en het beloop van PSD en PSA, en of er verschillen kunnen worden gevonden tussen de associaties met deze twee syndromen.

In Hoofdstuk 2 hebben we een systematisch literatuuronderzoek en een metaanalyse gedaan naar kenmerken op beeldvorming (oftewel imaging markers) geassocieerd met PSD en PSA. We includeerden in totaal 149 studies die aan de criteria voldeden. In een meta-analyse onderzochten we de associatie tussen PSD of PSA en de zijde, het type en de locatie van de laesie, ook gestratificeerd per studie fase (acute, post-acute, chronische fase na de beroerte). Associaties met andere imaging markers (zoals witte stof hyperintensiteiten (WMH), atrofie, laesie volume, aantal laesies, cerebrale microbloedingen) werden kwalitatief onderzocht om zo een volledig overzicht te geven. In de post-acute fase (0.5 - 6 maanden na de beroerte) was PSD geassocieerd met frontale laesies en laesies in de basale ganglia. Er werden geen associaties gevonden met de zijde van de laesie (links of rechts), of het type van de laesie (ischemisch of hemorrhagisch). PSA was frequenter na een hersenbloeding in de acute fase ( $<15$ dagen), terwijl het frequenter was na een herseninfarct in de post-acute fase na de beroerte. PSA was niet afhankelijk van de zijde of de locatie van de laesie maar de hoeveelheid beschikbare PSA studies was beperkt. De huidige bevindingen suggereren dat locatie van de laesie, in plaats van zijde of type, een belangrijke risicofactor is voor PSD in de post-acute fase na de beroerte. Daarentegen was laesie type in plaats van locatie of zijde een belangrijke risicofactorvoor PSA, maar meer PSA studies zijn nodig om dit te kunnen bevestigen. 
In Hoofdstuk 3 worden de rationale en het design van de Cognitie en Affect na Stroke (beroerte) een Prospectieve Evaluatie van Risicofactoren (CASPER) gepresenteerd. De CASPER studie is een eenjarige prospectieve follow-up studie van een cohort dat een beroerte heeft doorgemaakt. Deze studie is uitgevoerd om voorspellers van vasculaire cognitieve beperking, depressie en apathie na een beroerte te bestuderen. Voor deze studie werden 250 patiënten geïncludeerd die een ischemische of hemorrhagische beroerte hebben gehad. De baseline meting werd drie maanden na de beroerte ingepland en bestond uit een bloedafname, registratie van socio-demografische informatie, een neuropsychologisch onderzoek en een neuropsychiatrisch interview. Ook werd er een structurele 3-Tesla Magnetische Resonantie Imaging (MRI) van het brein gemaakt. Het neuropsychologisch onderzoek en neuropsychiatrisch interview werden herhaald tijdens de 6-maand en 12-maand follow-up meting.

In Hoofdstuk 4 hebben we de temporele relaties tussen vermoeidheid na een beroerte (post-stroke fatigue, PSF) en PSD en tussen PSF en PSA bestudeerd. Het effect van PSF drie maanden na de beroerte op het beloop van depressieve en apathische symptomen werd gemeten over een periode van twaalf maanden. Daarnaast hebben we de omgekeerde richting bestudeerd door het effect van PSD (ofPSA) drie maanden na de beroerte op het beloop van vermoeidheidssymptomen te onderzoeken. De resultaten toonden een wederzijdse associatie tussen PSF en PSD, maar PSA was niet geassocieerd met PSF. Niveaus van PSF en PSD bleven stabiel over tijd. Het tijdig opsporen van deze aandoeningen is dan ook belangrijk in patiënten met een beroerte, omdat PSF en PSD de neiging hebben om te persisteren.

In Hoofdstuk 5 hebben we de rol van premorbide persoonlijkheidskenmerken in de ontwikkeling en het beloop van symptomen van depressie en apathie na een beroerte onderzocht. Op baseline (drie maanden na de beroerte) vonden we een associatie tussen hoog neuroticisme en hoge niveaus van depressieve symptomen en dit bleef stabiel over een periode van 12 maanden. Laag in extraversie (introversie) was geassocieerd met hoge niveaus van apathie op baseline en deze associatie bleef ook stabiel over tijd. Deze bevindingen tonen het belang aan van het inachtnemen van persoonlijkheidskenmerken als potentiële kwetsbaarheidsfactoren voor PSD en PSA.

In Hoofdstuk 6 hebben we de associatie bestudeerd tussen laesie-gerelateerde imaging markers en markers van vasculaire en degeneratieve achtergrondschade en de ontwikkeling van PSD en PSA. Deze resultaten toonden aan dat globale hersenatrofie en ernstige cerebral small vessel disease (CSVD) pathologie op MRI (ziekte van de kleine hersenvaten), maar niet laesie-gerelateerde markers, geassocieerd waren met het ontwikkelen van PSA in het eerste jaar na de beroerte, 
onafhankelijk van co-morbide PSD en het volume van de beroerte. Globale brein atrofie en medium cSVD pathologie op MRI waren geassocieerd met het ontwikkelen van PSD, na correctie voor het laesie volume en depressie in de voorgeschiedenis. Na correctie voor co-morbide PSA bleef alleen medium cSVD pathologie op MRI significant geassocieerd met PSA. Dit suggereert dat atrofie en ernst van cSVD pathologie belangrijke voorspellers zijn voor het ontwikkelen van PSA, en in mindere mate voor PSD.

In Hoofdstuk 7 hebben we longitudinale analyses uitgevoerd om de invloed van "any Vascular Cognitive Impairment (VCI)" (een cognitieve beperking ten gevolge van vasculaire hersenpathologie in tenminste één cognitief domein) en ernst van $\mathrm{VCl}$ (cognitieve beperking in meerdere cognitieve domeinen versus een beperking in een enkel cognitief domein) op het beloop van depressie en apathie symptomen over tijd te onderzoeken. "Any VCl" voorspelde een toename in niveaus van apathie over de periode van drie tot vijftien maanden na de beroerte en deze was het meest uitgesproken voor patiënten met VCl in meerdere cognitieve domeinen. Domein-specifieke analyses toonden aan dat voornamelijk executief dysfunctioneren en vertraagde informatie verwerkingssnelheid geassocieerd waren met een toename in apathie symptomen over tijd. Geen van de cognitieve variabelen voorspelden het beloop van depressieve symptomen. Deze resultaten impliceren dat hoewel apathie symptomen wellicht afwezig zijn in de eerste maanden na de beroerte, deze symptomen wel kunnen ontwikkelen over tijd in patiënten met $\mathrm{VCl}$.

In Hoofdstuk 8 hebben we onderzocht of executief dysfunctioneren (ED) een subtype van PSD onderscheidt door te bestuderen of het samen voorkomen van ED en PSD in een depressie-executieve dysfunctie subtype (DES) geassocieerd is met verschillende klinische en imaging correlaten van het brein en een slechtere prognose. De resultaten lieten zien dat het DES-subtype geassocieerd is met een groter witte stof laesie volume, een hogere frequentie van oude infarcten, meer globale brein atrofie, en een hogere totale cSVD brein pathologie score op MRI drie maanden na de beroerte. Longitudinale analyses lieten zien dat DES geassocieerd was met een minder voorspoedig beloop, met een niet-verbeterend patroon van slechtere cognitieve prestaties in vergelijking met patiënten met enkel ED en chronisch hoge niveaus van depressie in vergeljking met patiënten met alleen PSD. Onze bevindingen suggereren dat DES geassocieerd is met meer gegeneraliseerde (vasculaire) hersenpathologie, wat bewijs is voor een sterke biologische oorsprong voor depressie in de DES groep in vergelijking met patiënten met alleen PSD. 
Hoofdstuk 9 bevat een algemene discussie van onze bevindingen gepresenteerd in dit proefschrift. Daarnaast werden er methodologische overwegingen en conceptuele en klinische implicaties besproken en aanbevelingen voor toekomstig onderzoek werden gegeven. Daarnaast bevat dit laatste hoofdstuk een Engelse en Nederlandse samenvatting van de belangrijkste bevindingen, een kennisvalorisatie, een dankwoord, een publicatielijst en informatie over de auteur.

In het algemeen hebben de studies in deze dissertatie bewijs geleverd dat er verschillende factoren betrokken zijn bij de ontwikkeling van PSD en PSA en deze zijn van biologische en psychosociale aard. Deze kennis verbetert ons begrip van de pathofysiologie van deze entiteiten en ondersteunt het idee dat PSA kan voorkomen als een onafhankelijk syndroom, in afwezigheid van PSD. Verschillen in kwetsbaarheidsfactoren tussen patiënten zijn belangrijk om in overweging te nemen, door het aanbieden van behandeling op maat (patiënt-specifiek). Vroegere detectie van deze condities en beter onderscheid tussen PSD en PSA leidt hopelijk tot de ontwikkeling van behandelingsstrategieën en een betere klinische prognose na een beroerte. 


\section{KNOWLEDGE VALORIZATION}

The aim of this thesis was to examine the association of biological and psychosocial risk factors on the development and course of post-stroke depression (PSD) and post-stroke apathy (PSA), and whether differences could be found between the associations with these two syndromes. This valorization paragraph describes how the results of this thesis are of societal relevance and economic application.

Mrs. $K$ is a 69-year-old married woman who suffered from a stroke in her right hemisphere. Shortly after the stroke, her clinical symptoms were nausea and vomiting, sensations in the left corner of her mouth and in the fingers of her left hand. These symptoms were present for two weeks and then disappeared slowly. After ten weeks Mrs. K has an appointment with her neurologist. She tells him that she feels fine, and experiences no residual complaints anymore from the stroke. When the neurologist asks her husband how he experiences the situation, he answers: "My wife changed a lot since she suffered from the stroke. Since the stroke she is sleeping a lot during the day. She does not perform in any household chores and is also relatively inactive in comparison to her activities before the stroke, when she used to swim very often, which she no longer cares to do. Also, her social life is very limited at the moment. She is not interested in meeting with other people, except for our sons."

This case describes an example of a stroke patient suffering from apathy. However, she does not report this as a complaint to her neurologist, though her husband is worried about her passive behavior and lack of interest. Will the neurologist recognize the behavior of this patient as apathy?

\section{SOCIETAL RELEVANCE}

Stroke is a leading cause of disability in the world. In the Netherlands, each year around 41,000 individuals suffer from a stroke, and currently more than 240,000 individuals are estimated to live with the consequences of stroke every day. As a result of the aging population, the prevalence of stroke will increase steadily, from 186,000 in 2011 to 343,000 in 2040 . Currently, the costs associated with care for stroke patients are approximately $2.5 \%$ of the total health care costs. As the life expectancy after stroke has been increasing due to better acute stroke treatment, the number of stroke survivors dealing with the consequences of stroke is increasing as well.

The residual consequences of stroke vary between patients, but can include physical impairments (i.e. paresis), speech difficulties, visual impairment, cognitive impairment, changes in personality, and the occurrence of neuropsychiatric 
symptoms. These factors all have a huge impact on stroke survivors and their caregivers, family, and other close relatives. Neuropsychiatric symptoms, like depression and apathy, occur in around a third of stroke survivors and have a negative influence on functional outcome and quality of life. As health-care professionals often do not recognize these neuropsychiatric consequences timely, several stroke survivors have unmet treatment needs.

As loss of motivation plays an important role in both apathy and depression, there is large overlap in clinical symptomatology and several studies found also overlap in risk factors between these syndromes. Despite this overlap, there is also some evidence suggesting that different risk factors may be involved in the development of PSD and PSA. However, risk factors associated with PSA are studied less frequently compared with PSD and only few longitudinal studies are available. Also, studies examining and comparing risk factors involved in the development of both PSD and PSA are scarce. Therefore, it is currently poorly understood if and how both biological and psychosocial factors have a differential influence on the development and course of PSD and PSA over time. A better understanding of the role of these risk factors is important, as for developing treatment strategies for PSD and PSA, a better understanding of the underlying pathophysiological mechanisms is required. Also, a better understanding of the etiological factors involved in these syndromes may result in better recognition, distinction, and earlier detection of PSD and PSA. Eventually, this could have a positive effect on quality of life and clinical outcome of stroke survivors, as this knowledge may help improving treatment possibilities. By providing patient-tailored care options for patients at risk for developing PSD and PSA, the prevalence of PSD and PSA will hopefully reduce, which will eventually also lower the health care costs and societal burden associated with it.

\section{TARGET AUDIENCE}

The findings presented in this thesis are relevant for several target groups, including stroke survivors and their relatives, health care professionals, and researchers.

The results are relevant for stroke survivors and their relatives, as the studies presented in this thesis provide more information about risk factors that are involved in the development of PSD and PSA, and also how these influence the course of PSD and PSA over time. A better explanation and psycho-education with respect to the risk of developing PSD and PSA to stroke survivors and their relatives is important, as currently a lot of them are not aware that these syndromes can develop as a consequence of their brain damage, also at a later stage during the chronic stroke phase. The current development of specialized stroke after-care 
clinics, which have specific expertise regarding neuropsychiatric symptoms after stroke, like PSD and PSA, are therefore a good and promising development, as these clinics can provide this psycho-education. Therefore, we deem the findings of this thesis of particular relevance to these specialized stroke after-care clinics.

All health care professionals (neurologists, rehabilitation physicians, psychiatrists, general practitioners, and (neuro)psychologists) treating stroke survivors are important stakeholders as well, as they are the ones who need to inform stroke survivors about the risk of developing symptoms of depression and apathy after stroke, and which risk factors are involved in the development of these syndromes. The results of this thesis have shed more light on the risk factors that play an important role in the development and course of apathy and depression and shows that the risk factors being involved are different to some extent for PSD and PSA. More knowledge about the etiology of PSD and PSA will help to recognize these conditions by health care professionals and identify patients (timely) who are at risk to develop PSD or PSA. Better diagnostic distinction between PSD and PSA is important, as these conditions seem to benefit from different treatment strategies.

The findings resulting from this thesis are also relevant for researchers in the stroke field, as they shed more light on the biological and psychosocial factors that are involved in the etiology of PSD and PSA, which improves the understanding of the pathophysiology of these syndromes. This information is particularly important for researchers who examine possible pharmacological and psychotherapeutical treatment strategies for PSD and PSA, as the results indicate that PSA can occur as an independent syndrome, independent of PSD, and has its own associated risk factors that influence the development and course of this syndrome. In addition, the results may also be of use for researchers who work with other patient populations in which both apathy and depression are frequent (like Parkinson's disease, Alzheimer's disease and other types of dementia, or traumatic brain injury), as some of the associations found in this thesis might also hold for these other disease populations.

\section{INNOVATION AND PRODUCTS}

The CASPER study measured a range of 3-Tesla structural brain MRI markers, both volumetric and visual. The semi-automatic volumetric assessment of white matter hyperintensities and manual segmentation of stroke lesion tissue provided a precise measure, which made it possible to study subtle differences and also to study the role of both lesion-related markers (location, volume, laterality) and generalized background brain pathology (total cerebral small vessel disease 
burden, global brain atrophy, white matter hyperintensity volume, number of old infarcts or lacunes, and number of cerebral microbleeds). This made it possible to compare the influence of certain MRI markers on the development of PSD and PSA. The main product of this thesis is the implications that the findings have for the understanding of the pathophysiology of PSD and PSA. The findings provide evidence that both biological and psychosocial factors are involved in the development of PSD and PSA. Also, differences in associated risk factors between PSD and PSA suggest that PSA can also occur as an independent syndrome, in absence of depressive symptoms. These findings are important for researchers developing treatment strategies for PSD and PSA, as a better understanding of the complex multifactorial origin of these conditions is needed for successful treatment.

Participants of the study were updated about the study progress through newsletters. Also, the results in this thesis were presented at several international and national congresses.

\section{IMPLEMENTATION}

The clinical importance of this thesis involves the differentiation between risk factors being involved in the development of PSD and PSA, which were of both biological and psychosocial origin. The knowledge derived from the studies presented in this thesis will be the base for research into the role of biological and psychosocial predictors of PSD, PSA, and VCI. The results presented in this thesis show that it is important to consider the role of both biological and psychosocial factors, and studying their inter-relationships and interactions is another future goal, as this is needed to unravel the complex multifactorial origin of PSD and PSA. Also other biomarker data were collected for CASPER (inflammatory markers and epigenetics), which were not part of the present thesis but will be used for future studies, as these are also likely to be important factors influencing the development and course of PSD and PSA. In addition, the CASPER study joined the STROKOG consortium (https://cheba.unsw.edu.au/group/strokog), which has built a database with more than 12,000 stroke patients. This large dataset yields new opportunities for studying subtle changes, which require large datasets and will likely yield important results concerning factors associated with PSD and PSA. Also, participants of CASPER were approached for participation in our long-term follow-up study, for which we are currently collecting data. For this study, we are collecting data at 3 years post-stroke. These long-term data can provide interesting findings with respect to PSD and PSA as potential risk factors for mortality and the development of vascular dementia. 


\section{HOW COULD MRS. K BENEFIT FROM THE RESULTS OF THIS THESIS?}

The neurologist of Mrs. K has read some articles on apathy after stroke and realizes that this is a frequent complaint after stroke, which is often confused with depression. He administers a semi-structured questionnaire based on DSM-5 criteria to find out if Mrs. K has depressed feelings, but that was not the case. The neurologist explains to Mrs. K and her husband that apathy is a frequent complaint after stroke and that it is sometimes part of a depressive disorder, but not necessarily. He tells them that there are currently no pharmacological treatment options for apathy available. He advises the husband to keep motivating Mrs. K to stay active and to help her setting goals, because that is difficult for her. The neurologist also decides to refer them to a neuropsychologist, who can help the couple dealing with this new situation. 
Addendum 


\section{DANKWOORD (ACKNOWLEDGEMENTS)}

De afgelopen drie jaar zijn voorbij gevlogen en met veel plezier heb ik gewerkt aan de totstandkoming van dit proefschrift. Het was een bijzondere ervaring, waarin ik veel heb geleerd. Dit mooie resultaat heb ik niet in mijn eentje bereikt en daarom wil ik ik graag alle mensen bedanken die hieraan een bijdrage hebben geleverd.

Allereerst wil ik mijn promotieteam bedanken voor de expertise en begeleiding.

Mijn promotor, Prof. Dr. Frans Verhey, beste Frans. Bedankt dat je me de kans hebt geboden om de afgelopen jaren aan dit onderzoek te mogen werken en om mezelf te ontwikkelen tot de wetenschapper die ik nu ben. Zowel je klinische als wetenschappelijke kennis waren van onschatbare waarde voor het project. Bedankt voor de fijne samenwerking.

Mijn co-promotoren, dr. Pauline Aalten en dr. Julie Staals.

Beste Pauline, vanaf het begin van mijn promotietraject gaf je me het gevoel dat jij er alle vertrouwen in had dat ik aan het eind van mijn promotietraject een proefschrift zou afleveren waar we trots op konden zijn; en ik heb het gevoel dat het gelukt is! Jouw enthousiasme en support speelden hierin een belangrijke rol. Het is bewonderenswaardig hoe je je werk als onderzoeker en studieadviseur combineert en vaak in de (late) avonduren bezig was om mijn stukken na te kijken, of bijna 6000 abstracts te screenen. Ik wil je bedanken voor je begeleiding, het vetrouwen, de fijne overleggen waarin we ook andere dingen dan het onderzoek konden bespreken, en voor de prettige samenwerking.

Beste Julie, wat heb ik veel van je geleerd. In ruil voor wat zakjes drop was je bereid om voor de CASPER studie bijna 200 MRI scans samen te bekijken (de meeste zelfs twee keer) en het was voor mij erg leerzaam. Je hielp bij het vinden van de deelnemers en dacht mee op alle fronten van het onderzoek. Als ik een nieuw manuscript rondstuurde was je bijna altijd de eerste die er naar had gekeken. Hier stond dan vaak in dat het wat aan de lange kant was (omdat psychologen in tegenstelling tot neurologen soms wat lang van stof kunnen zijn). Je expertise was van onschatbare waarde. Bedankt voor de prettige samenwerking.

Dank aan alle leden van de beoordelingscommissie, Prof. dr. Hofman, Prof. dr. Visser-Meily, Prof. dr. de Leeuw, Dr. Duits en Dr. Leentjens, voor het lezen en beoordelen van mijn proefschrift.

Een bijzonder woord van dank aan de andere leden van het CASPER-team. 
Dr. Köhler, beste Seb, jij was de spil van het onderzoek en jouw kennis en vaardigheden tilden onze artikelen naar een hoger level. Bedankt voor alles wat ik van je heb mogen leren in de afgelopen jaren, voor je statistische kennis en hulp, de interessante gesprekken over de bevindingen die we deden, en voor je support als ik een praatje moest geven op een congres. Jouw passie, gedrevenheid en enthousiasme werken aanstekelijk, bedankt voor de fijne samenwerking!

Prof. dr. Robert J. van Oostenbrugge, beste Robert. Bedankt voor je expertise en kritische blik op alle artikelen. Je hielp ons om de soms ingewikkelde neuropsychologische resultaten begrijpelijk te houden voor clinici. Ondanks je drukke agenda had je toch tijd om samen met mij zo'n 100 MRI scans te beoordelen. Bedankt!

Syenna, lieve roomie, en mijn partner op het project. Dankzij jouw harde werken aan het opzetten van de CASPER studie, een enorme klus waarbij aan ieder detail gedacht is, kon ik als stagiaire en daarna als AlO direct van start om samen met jou deelnemers te vinden en de data te verzamelen. Dit ging niet altijd zonder slag of stoot, maar we bleven positief en vierden iedere geïncludeerde deelnemer. Met jou kon ik als geen ander sparren over studie-gerelateerde kwesties en over nog zoveel meer! Veel lief en leed hebben we de afgelopen jaren met elkaar gedeeld, maar vooral ontzettend gelachen! Bedànk meadje!

Hartelijk dank aan alle deelnemers en naasten die hebben meegewerkt aan de CASPER studie. Zonder jullie bereidheid en enthousiasme om een bijdrage te leveren aan deze studie was het totstandkomen van dit proefschrift nooit mogelijk geweest. Ook dank ik alle stagiaires en onderzoeksassistenten die zich hebben ingezet voor de studie: Dynah, Danique (op jou kom ik later nog even terug!), Lieke, Loes, Esmee, Sascha, Janneke, Tessa, Lise, Ileana, Anne en Wies. Tanja, bedankt voor alle telefonische naaste-interviews die je voor ons hebt gedaan. Joost, bedankt voor je hulp bij de dubbele datainvoer.

Bedankt aan alle medewerkers van de afdeling neurologie van het MUMC+, in het bijzonder de baliemedewerkers van verpleegafdeling C5 en CVA-verpleegkundige Anouk van der Kwaak, voor de hulp bij het benaderen van de deelnemers.

Ook hartelijk dank aan Zuyderland Medisch Centrum te Sittard en Heerlen, in het bijzonder Roger Nieling en Tiny Simons-Sporken, voor jullie hulp bij de inclusie voor de CASPER studie. 
De medewerkers van de afdeling radiologie, in het bijzonder Martijn Wolters, May Wong, Harm van de Haar, Marc Geerlings en Jos Slenter, bedankt voor al jullie expertise en hulp bij het verkrijgen, anonimiseren, bewerken en analyseren van onze MRI data.

Many thanks to Mercedes for her contribution to the systematic review and metaanalysis. It resulted in a great publication! Robin Wolz, thank you for the acquisition of the hippocampal volume data.

Een speciaal woord van dank aan lliana Boshoven van AgileColor.com voor de cover en lay-out van het boekje. Dankzij jou ziet het proefschrift er prachtig uit!

Dymphie in de Braek, Chantal Geusgens en Marjolein de Vugt. Door jullie is bij mij het enthousiasme voor de klinische praktijk altijd gebleven. Bedankt voor alles wat ik van jullie "neuropsychologen" heb mogen leren in de afgelopen jaren.

Alle (oud-)collega's van de afdeling Psychiatrie en Neuropsychologie wil ik bedanken voor de fijne samenwerking, gezellige sfeer op de afdeling en de leuke uitjes en congressen.

Mijn paranimfies Danique en Liselot! Wat ben ik trots dat jullie tijdens de verdediging achter mij staan!

Lieve Danique, bijna 4 jaar geleden leerde ik je kennen als mijn stagiaire-partner op de CASPER studie. Daarna heb je ook als onderzoeksassistent mij op alle fronten geholpen bij de studie en was je af en toe mijn redder in nood! Ook hebben we samen vele uren doorgebracht in het MRI computerhok waar we de hele dag zaten te "kleuren" onder het genot van Sky radio christmas! Inmiddels ben je alweer ruim een jaar bezig met je eigen PhD en zijn we zelfs roomies. Lieve Danique, bedankt voor alle mentale support, je hulp, je gezelligheid. Voor alles. Zonder jou was het lang niet zo leuk geweest!

Lieve Liselot, in september 2014 begonnen we beide aan onze PhD, als roomies! Samen verkenden we het AIO wereldje, gingen we naar alle cursussen. Jij overtuigde me dat ik best even pauze kon nemen als ik weer eens (te) druk bezig was, samen even een rondje wandelen, een koffietje doen. Bedankt voor alle gezelligheid, voor de leuke logeerpartijtjes, chocola-uitwisselingen, bioscoop bezoekjes, en met als hoogtepunt toch wel de leuke trip naar Florence dit jaar, samen met Anne, Leonie, en Angélique. Wat hebben we gelachen, lekkere cheesecake gehad (en ook vieze), een ruime hoeveelheid zon, lekker eten, veel wijn.... Dankjewel lieve Liselot, je bent een topper! 
Mijn buurvrouwen van de UNS40: Alieske, Isabelle, Whitney en Lotte. Jullie zijn geweldig! Wat is het fijn om de laatste loodjes van je PhD te kunnen delen met zulke supportgevende collega's! Het maakte het allemaal een stuk draaglijker. Alieske, samen met jou naar Berlijn en Florence was een feestje, wat een gezellige tijd, konden we het nog maar eens overdoen! Isabelle, je vrolijkheid en enthousiasme en altijd goede raad maken je een topcollega. Whitney, super leuk dat je als coauteur op een van de artikelen staat! Jouw radiologische kennis kwam goed van pas. Bedankt voor alle gezellige momenten! Lotte, jij staat altijd voor iedereen klaar! Bedankt dat je nog een blik op mijn discussie hebt willen werpen voor de laatste grammaticale punten op de i.

Angélique, ruim een jaar geleden begon je als AIO maar het voelt alsof ik je al veel langer ken. Uren hebben we al samen in de trein gezeten op het traject WeertMaastricht, maar we zijn nóóit uitgepraat. Je bent altijd geïnteresseerd, wist altijd de status van mijn artikelen, je bent een topper!

Leonie, altijd fijn om met jou even onze ervaringen uit te wisselen, omdat je net als ik een 1+3 constructie doet. Dankjewel voor je support en gezelligheid!

Mijn ex-roomies, Kay, Inge en Babette. Wat een gezellige drukte was het soms hè op onze kamer! Het was fijn om ervaringen uit te wisselen en ondanks dat we aan verschillende projecten werkten konden we bij elkaar terecht voor advies. Kay, bedankt voor je hulp en raad bij het uitvoeren van mijn systematische review en bij allerlei andere zaken. Leuk dat je me in een sneltreinvaart wat van Londen kon laten zien! Inge, je staat altijd voor iedereen klaar en hebt ook geholpen bij het includeren van onze deelnemers. Babette, bedankt voor de gezellige tijd, veel succes met de laatste loodjes van je proefschrift.

Rosalie, Lizzy, Willemijn en Joany. Jullie waren de senior AlOs bij wie ik altijd terecht kon met vragen. Super fijn als iemand met je meedenkt en hulp biedt bij de laatste loodjes van het promoveren! Bedankt voor alle gezelligheid op het werk, in de trein en op congres.

Nico, zonder jouw hulp was het uitvoeren van het onderzoek praktisch onmogelijk. Wat een verademing was het toen we ons excel bestand konden vervangen door filemaker, dit scheelde een hoop werk en vooral stress! Bedankt voor al je hulp bij computerproblemen, je stond altijd voor me klaar. Daniëlle en Els, jullie waren altijd bereid om te helpen, bedankt! 
Renske en Eleana, collega AlOs van de neurologie. Renske, het was altijd fijn en gezellig om onze PhD ervaringen te delen en om elkaar tegen te komen bij cursussen. We waren tegelijkertijd bezig met onze laatste loodjes en nu gaan we vlak na elkaar verdedigen. Fijn dat ik nog even bij jou kan afkijken voordat ik zelf aan de beurt ben ;-)! Samen op congres in Amsterdam en Praag was onwijs gezellig!

Eleana, als collega binnen het stroke onderzoek was het fijn om met je samen te werken, om ervaringen te delen en om bij te kletsen over vanalles en nog wat.

Sanne, Anne, Niky en Danique (coffeelovers). Wij waren de harde kern die overbleef in Maastricht na onze stage periode als MUMC chicks. Wat een toppers zijn jullie! Bedankt voor de lunches, koffiedates, en etentjes de afgelopen tijd. Met jullie is het altijd een berg gezelligheid. Hopelijk volgen er nog vele afspraakjes!

Lieve vriendinnen, vrienden en schoonfamilie, het was wellicht een raadsel voor jullie wat dat promoveren nou precies inhield, maar hopelijk is het met het zien van dit boekje nu wat duidelijker! Bedankt voor jullie interesse in de voortgang van mijn onderzoek en voor jullie gezelligheid en afleiding wanneer dat soms nodig was. Hopelijk volgen er nog vele leuke en gezellige feestjes, etentjes, borrels, uitjes en andere momenten samen!

Lieve Inge, mijn grote zus, en schoonbroer Martijn. Bedankt voor jullie interesse en voor de leuke dingen die we samen doen. Dat ik dit jaar getuige mocht zijn op jullie bruiloft was een hele eer!

Lieve pap en mam. Door jullie ben ik geworden wie ik nu ben. Bedankt voor alles wat jullie me hebben meegegeven en voor alle steun en interesse die jullie altijd lieten en laten blijken. Door jullie heb ik geleerd om te genieten van alles wat ik mee mag maken. Ik weet dat jullie trots op mij zijn! Het is fijn om zo'n fijn "thuis" te hebben.

Tot slot, liefste Patrick. Jij bent zonder twijfel de allerbelangrijkste reden dat het mij gelukt is om dit proefschrift af te ronden. Jij wist altijd precies wat ik nodig had als ik even gestresst was. Jouw nuchtere kijk op dingen werkt verhelderend. Bedankt voor je steun en hulp, maar vooral bedankt voor wat wij samen hebben. Op nog vele mooie jaren! 
Addendum 


\section{THESIS DEFENSES FROM MHENS (SCHOOL FOR MENTAL HEALTH AND NEUROSCIENCE)}

\section{3}

Rob Havermans: Bipolar disorder in daily life; Mood and cortisol responses to naturally occurring events. Supervisor: Prof.dr. M. de Vries; Co-Supervisor: Dr. N. Nicolson.

Véronique Moers-Hornikx: Deep brain stimulation and the cerebellum. Supervisors: Prof.dr. J. Vles / Prof.dr. Y. Temel; Co-Supervisor: Dr. G. Hoogland.

Nicole Veldhorst-Janssen: Intranasal delivery of rapid acting drugs. Supervisors: Prof.dr. M. Marcus / Prof.dr. C. Neef; Co-Supervisor: Dr. P.H. van der Kuy.

Stéphanie Knippenberg: Vitamin D and Multiple Sclerosis: immunological and clinical outcome. Supervisor: Prof.dr. J. Cohen-Tervaert; Co-Supervisors: Dr. J. Damoiseaux / Dr. Y. Bols.

Erik D. Gommer: Dynamic Cerebral Autoregulation: from methodology towards clinical application. Supervisors: Prof.dr. W.H. Mess / Prof.dr. R.B. Panerai, UK; Co-Supervisor: Dr.ir. J.P.H. Reulen.

Olga A.H. Reneerkens: Can PDE inhibition improve cognition ? Translational insights. Supervisor: Prof. dr. H.W.M. Steinbusch; Co-Supervisor: Dr. J. Prickaerts.

Lyzel S. Elias-Sonnenschein: Clinical and biomarker correlates of genetic risk factors for Alzheimer's disease. Supervisor: Prof.dr. F.R.J. Verhey; Co-Supervisor: Dr. P.J. Visser.

Diego F. Mastroeni: Epigenetic Dysregulation and the Pathophysiology of of Alzheimer's Disease. Supervisors: Prof.dr. H.W.M. Steinbusch / Prof.dr. P.D. Coleman, Sun City, Arizona; Co-Supervisors: Dr. B.P.F. Rutten / Dr. D.L.A. van den Hove.

Leonidas Chouliaras: Epigenetic Regulation in Aging and Alzheimer's disease: A translational perspective. Supervisor: Prof.dr. H.W.M. Steinbusch; Co-Supervisors: Dr. B.P.F. Rutten / Dr. D.L.A. van den Hove.

Liesbeth Knaepen: Perinatal events and altered pain sensitivity in later life. Supervisors: Prof.dr. E.A.J. Joosten / Prof.dr. D. Tibboel, EUR; Co-Supervisor: Dr. J. Patijn.

Marisela Martinez-Claros: Hippocampal plasticity and corticosterone: From dendrites to behaviour. Supervisor: Prof.dr. H.W.M. Steinbusch; Co-Supervisors: Dr. J.L. Pawluski / Dr. J. Prickaerts.

Marcus D. Lancé: A circle of improvement in bleeding management: from laboratory to clinic and back. Supervisors: Prof.dr. M.A.E. Marcu / Prof.dr. J.W.M. Heemskerk; Co-Supervisor: Dr. Y.M.C. Henskens.

Hilde Braakman: Imaging the brain; neuronal correlates of cognitive impairment in children with frontal lobe epilepsy. Supervisors: Prof.dr. A.P. Aldenkamp /Prof.dr. J.S.H. Vles; Co-Supervisors: Dr.ir. W.H. Backes / Dr. P.A.M. Hofman.

Willem H. van Zwam: Aneurysmal subarachnoid hemorrhage: imaging strategies and costeffectiveness aspects in diagnostic work-up and post-therapeutic follow-up. Supervisors: Prof. dr. J.T. Wilmink / Prof.dr. J.E. Wildberger; Co-Supervisor: Dr. P.A.M. Hofman.

Klara De Cort: The Pathogenesis of Panic Disorder. Supervisors: Prof.dr. I. Myin-Germeys / Prof.dr. E.J.L. Griez; Co-Supervisors: Dr. K.R.J. Schruers / Dr. I. Van Diest, Leuven.

Kim van Wijck: Mind the Gap; experimental studies on splanchnic hyperfusion and gastrointestinal integrity loss in man. Supervisors: Prof.dr. W.A. Buurman / Prof.dr. C.H.C. Dejong; Co-Supervisor: Dr. K. Lenaerts. 
Yvette Roke: Antipsychotic-induced hyperprolactinemia in children and adolescents with mainly autism spectrum disorders. Prevalence, symptoms, clinical consequences and genetic risk factors. Supervisors: Prof.dr. P.N. van Harten / Prof.dr. J.K. Buitelaar (RUN); Co-Supervisor: Dr. A. Boot (UMCG).

Fleur Goezinne: Retinal detachment surgery: pre and postoperative prognostic factors. Supervisors: Prof.dr. F. Hendrikse / Prof.dr. C.A.B. Webers; Co-Supervisor: Dr. E.C. La Heij (Amsterdam).

Ralph L.J.G. Maassen: The Merits of Videolaryngoscopy during Glottic Visualisation for Endotracheal Intubation. Supervisors: Prof.dr. M. Marcus / Prof.dr. A. van Zundert (University of Queensland).

Maria J. de Sousa Guerreiro: The role of sensory modality in age-related distraction Supervisor: Prof. dr. C.M. van Heugten; Co-Supervisor: Dr. P.W.M. van Gerven.

Ine Rayen: Effects of developmental fluoxetine exposure on neurobehavioral outcomes. Supervisor: Prof.dr. H.W.M. Steinbusch; Co-Supervisors: Dr. J.L. Pawluski / Dr. T.D. Charlier (Ohio University, USA).

Nynke M.G. Bodde: Psychogenic non-epileptic seizures; a separate disorder or part of a continuum? Supervisors: Prof.dr. R. van Oostenbrugge / Prof.dr. K. Vonck (UZ Gent); Co-Supervisors: Dr. R. Lazeron / Dr. A. de Louw (Epilepsiecentrum Kempenhaeghe, Heeze).

Alejandro M. Gomez: Novel strategies for making myasthenia less gravis: targeting plasma cells and the neuromuscular junction. Supervisor: Prof.dr. M.H. De Baets; Co-Supervisors: Dr. M. Losen / Dr. P. Martinez-Martinez.

Mohammad S. Rahnama'i: Prostaglandins and Phosphodiesterases in the Urinary Bladder Wall. Supervisors: Prof.dr. Ph. Van Kerrebroeck / Prof.dr. S. de Wachter (Universiteit Antwerpen); CoSupervisor: Dr. G. van Koeveringe.

Mariken B. de Koning: Studying biomarkers in populations at genetic and clinical high risk for psychosis. Supervisors: Prof.dr. T. Amelsvoort / Prof.dr. J. Booij (AMC).

Fabien Boulle: Epigenetic regulation of BDNF/TrkB signaling in the pathophysiology and treatment of mood disorders. Supervisors: Prof.dr. H.W.M. Steinbusch / Prof.dr. L. Lanfumey (Universiteit Parijs); Co-Supervisors: Dr. D. van den Hove / Dr. G. Kenis.

\section{4}

Iris Nowak-Maes: Tinnitus; assessment of quality of life \& cost-effectiveness. Supervisors: Prof.dr. M. Peters / Prof.dr. B. Kremer; Co-Supervisors: Dr. M. Joore / Dr. L. Anteunis.

Marjolein Huijts: Cognitive function in patients with cerebral small vessel disease. Supervisor: Prof.dr. R.J. van Oostenbrugge; Co-Supervisors: Dr. A.A. Duits / Dr. J. Staals.

Markus Gantert: Fetal inflammatory injury as origin of long term disease: Lessons from animal models. Supervisors: Prof.dr. B. Kramer / Prof.dr. L. Zimmermann; Co-Supervisor: Dr. A. Gavilanes.

Elke Kuypers: Fetal development after antenatal exposures: Chorioamnionitis and maternal glucocorticoids. Supervisors: Prof.dr. B.W. Kramer / Prof.dr. H.W. Steinbusch / Prof.dr. Suhas G. Kallapur (University of Cincinnati, Ohio, USA).

Pieter Kubben: Ultra low-field strength intraoperative MRI for Glioblastoma Surgery. Supervisor: Prof. dr. J.J. van Overbeeke; Co-Supervisor: Dr. H. van Santbrink.

Laura Baijens: Surface electrical stimulation of the neck for oropharyngeal dysphagia in Parkinson's disease: therapeutic aspects and reliability of measurement. Supervisor: Prof.dr. B. Kremer; CoSupervisor: Dr. R. Speyer, Townsville. 
Janneke Hoeijmakers: Small fiber neuropathy and sodium channels; a paradigm shift. Supervisor: Prof.dr. R.J. van Oostenbrugge; Co-Supervisors: Dr. C.G. Faber / Dr. I.S.J. Merkies.

Stephanie Vos: The Role of biomarkers in preclinical and prodromal Alzheimer's disease. Supervisor: Prof.dr. F.R. Verhey; Co-Supervisor: Dr. P.J. Visser.

Muriël Doors: The Value of Optical Coherence Tomography in Anterior Segment Surgery. Supervisors: Prof.dr. R.M. Nuijts / Prof.dr. C.A. Webers; Co-Supervisor: Dr. T.T.J.M. Berendschot.

Anneke Maas: Sleep problems in individuals with genetic disorders associated with intellectual disability. Supervisors: Prof.dr. I. Curfs / Prof.dr. R. Didden.

Sebastiaan van Gorp: Translational research on spinal cord injury and cell-based therapies; a focus on pain and sensorimotor disturbances. Supervisors: Prof.dr. B. Joosten / Prof.dr. M. van Kleef; CoSupervisors: Dr. J. Patijn /Dr. R. Deumens, KU Leuven.

Andrea Sannia: High risk newborns and brain biochemical monitoring. Supervisor: Prof.dr. J.S.H. Vles; Co-Supervisors: Dr. D. Gazzolo, Alessandria, Italy / Dr. A.W.D. Gavilanes.

Julie A.D.A. Dela Cruz: Dopamine mechanisms in learning and memory: Evidence from rodent studies. Supervisors: Prof.dr. H.W.M. Steinbusch / Prof.dr. R.J. Bodnar, New York; Co-Supervisor: Dr. B.P.F. Rutten.

René Besseling: Brain wiring and neuronal dynamics; advances in MR imaging of focal epilepsy. Supervisors: Prof.dr. A.P. Aldenkamp / Prof.dr.ir. W.H. Backes; Co-Supervisor: dr. J.F.A. Jansen.

Maria Quint-Fens: Long-term care after stroke; development and evaluation of a long-term intervention in primary care. Supervisors: Prof.dr. J.F.M. Metsemakers / Prof.dr. C.M. van Heugten / Prof.dr. M. Limburg, Almere; Co-Supervisor: dr. G.H.M.I. Beusmans.

Veronique Moulaert: Life after survival of a cardiac arrest; the heart of the matter. Supervisors: Prof.dr. J.A. Verbunt / Prof.dr. C.M. van Heugten / Prof.dr. D.T. Wade, Oxford, UK.

Feikje Smeets: The hallucinatory-delusional state: a crucial connection in the psychosis symptom network. Supervisor: Prof.dr. J. van Os; Co-Supervisor: Dr. T. Lataster.

Lies Clerx: Alzheimer's disease through the MR-eye; novel diagnostic markers and the road to clinical implementation. Supervisor: Prof.dr. F. Verhey; Co-Supervisors: Dr. P.J. Visser / P. Aalten.

Sonny Tan: The subthalamic nucleus in Parkinson's disease. Supervisors: Prof.dr. Y. Temel / Prof.dr. H.W.M. Steinbusch / Prof.dr. T. Sharp, Oxford, UK / Prof.dr. V. Visser-Vandewalle, Koln.

Koen van Boxem: The use of pulsed radiofrequency in the management of chronic lumbosacral radicular pain. Supervisors: Prof.dr. M. van Kleef / Prof.dr. E.A.J. Joosten; Co-Supervisor: Assoc. Prof.dr. J. van Zundert.

Jérôme Waterval: Hyperostosis cranialis interna. Supervisors: Prof.dr. J.J. Manni / Prof.dr. R.J. Stokroos.

Sylvie Kolfschoten-van der Kruijs: Psychogenic non-epileptic seizures; the identification of neurophysiological correlates. Supervisors: Prof.dr. A.P. Aldenkamp / Prof.dr. K.E.J. Vonck, Universiteit Gent; Co-Supervisors: Dr. J.F.A. Jansen / Dr. R.H.C. Lazeron, Kempenhaeghe.

Wouter Pluijms: Spinal cord stimulation and pain relief in painful diabetic: polyneuropathy, a translational approach. Supervisors: Prof.dr. M. van Kleef / Prof.dr. E.A. Joosten; Co-supervisor: Dr. C.G. Faber.

Ron Handels: Health technology assessment of diagnostic strategies for Alzheimer's disease. Supervisors: Prof.dr. F.R.J. Verhey / Prof.dr. J.L. Severens (EUR); Co-Supervisor: Dr. M.A. Joore / Dr. C.A.G. Wolfs.

Evelyn Peelen: Regulatory T cells in the pathogenesis of Multiple Sclerosis: potential targets for vitamin D therapy. Supervisors: Prof.dr. R.M.M. Hupperts / Prof.dr. J.W. Cohen Tervaert; CoSupervisor: Dr. J.G.M.C. Damoiseaux / Dr. M.M.G.L.Thewissen, Diepenbeek. 
Reint Jellema: Cell-based therapy for hypoxic-ischemic injury in the preterm brain. Supervisors: Prof. dr. B.W.W. Kramer / Prof.dr. H.W.M. Steinbusch; Co-Supervisor: Dr. W.T.V. Germeraad / Dr. P. Andriessen, Veldhoven.

Maria Wertli: Prognosis of Chronic Clinical Pain Conditions: The Example of Complex Regional Pain Syndrome 1 and Low Back Pain. Supervisors: Prof.dr. M. van Kleef; Co-Supervisor: Dr. F. Brunner, Zürich / Dr. R. Perez, VUmc.

Dagmar Zeef: An experimental model of Huntington's disease: Validation \& Stimulation. Supervisors: Prof.dr. Y. Temel / Prof.dr. H.W.M. Steinbusch; Co-supervisor: Dr. A. Jahanshahi.

Jeroen Decoster: Breaking Down Schizophrenia into phenes, genes and environment. Supervisors: Prof.dr. I. Myin-Germeys / Prof.dr. M. De Hert, KU Leuven; Co-Supervisor: Dr. R. van Winkel.

Eaja Anindya Sekhar Mukherjee: Fetal Alcohol Spectrum Disorders: exploring prevention and management. Supervisor: Prof.dr. L.M.G. Curfs; Co-Supervisor: Prof. S. Hollins, St. George's University of London, UK.

Catherine van Zelst: Inside out; On stereotype awareness, childhood trauma and stigma in psychosis. Supervisors: Prof.dr. Ph. Delespaul / Prof.dr. J. van Os.

Ibrahim Tolga Binbay: Extended Psychosis Phenotype in the Wider Social Environment. Supervisor: Prof.dr. J. van Os; Co-Supervisor: Dr. M. Drukker.

Frank Van Dael: OCD matters in psychosis. Supervisors: Prof.dr. J. van Os / Prof.dr. I. Myin-Germeys.

Pamela Kleikers: NOXious oxidative stress: from head toe too and back. Supervisors: Prof.dr. H.H.H.W. Schmidt / Prof.dr. H.W.M. Steinbusch; Co-Supervisor: Dr. B. Janssen.

José Luis Gerardo Nava: In vitro assay systems in the development of therapeutic interventions strategies for neuroprotection and repair. Supervisors: Prof.dr.med. J. Weis / Prof.dr. H.W.M. Steinbusch; Co-Supervisor: Dr. G.A. Brook, RWTH Aachen.

Eva Bollen: Cyclic nucleotide signaling and plasticity. Supervisors: Prof.dr. H.W.M. Steinbusch / Prof.dr. R. D'Hooge, KU Leuven; Co-Supervisor: Dr. J. Prickaerts.

\section{5}

Jessica A. Hartmann: A good laugh and a long sleep; Insights from prospective and ambulatory assessments about the importance of positive affect and sleep in mental health. Supervisor: Prof.dr. J. van Os; Co-Supervisors: C.J.P. Simons / Dr. M. Wichers.

Bart Ament: Frailty in old age; conceptualization and care innovations. Supervisors: Prof.dr. G.I.J.M. Kempen / Prof.dr. F.R.J. Verhey; Co-Supervisor: Dr. M.E. de Vugt.

Mayke Janssens: Exploring course and outcome across the psychosis-continuum. Supervisor: Prof.dr. I. Myin-Germeys; Co-Supervisor: Dr. T. Lataster.

Dennis M.J. Hernau: Dopayours is not dopamine: genetic, environmental and pathological variations in dopaminergic stress processing. Supervisor: Prof.dr. I. Myin- Germeys; Co-Supervisors: Prof.dr. F.M. Mottaghy / Dr. D. Collip.

Ingrid M.H. Brands: The adaptation process after acquired brain injury Pieces of the puzzle. Supervisors: Prof.dr. C.M. van Heugten / Prof.dr. D.T. Wade, Oxford UK; Co-Supervisors: Dr. S.Z. Stapert / Dr. S. Köhler.

Francesco Risso: Urinary and salivary S100B monitoring in high risk infants. Supervisor: Prof.dr. J.S.H. Vles; Co-Supervisors: Dr. D. Gazzolo, Genoa,Italy / Dr. A.W.D. Gavilanes.

Alessandro Borghesi: Stem and Progenitor Cells in Preterm Infants: Role in the Pathogenesis and Potential for Therapy. Supervisor: Prof.dr. L. Zimmermann; Prof.dr. B. Kramer; Co-Supervisors: Dr. D. Gazzolo, Genoa, Italy / Dr. A.W.D. Gavilanes. 
Claudia Menne-Lothmann: Affect dynamics; A focus on genes, stress, and an opportunity for change. Supervisor: Prof.dr. J. van Os; Co-Supervisors: Dr. M. Wichers / Dr. N. Jacobs.

Martine van Nierop: Surviving childhood new perspectives on the link between childhood trauma and psychosis. Supervisors: Prof.dr. I. Myin-Germeys / Prof.dr. J. van Os; Co-Supervisor: Dr. R. van Winkel.

Sylvia Klinkenberg: VNS in children; more than just seizure reduction. Supervisors: Prof.dr. J. Vles / Prof.dr. A. Aldenkamp; Co-Supervisor: Dr. H. Majoie.

Anouk Linssen: Considerations in designing an adult hearing screening programme. Supervisor: Prof. dr. B. Kremer; Co-Supervisors: Dr. L. Anteunis / Dr. M. Joore.

Janny Hof: Hearing loss in young children; challenges in assessment and intervention. Supervisors: Prof.dr. B. Kremer / Prof.dr. R. Stokroos / Prof.dr. P. van Dijk, RUG; Co-Supervisor: Dr. L. Antheunis.

Kimberly Cox-Limpens: Mechanisms of endogenous brain protection; Clues from the transcriptome. Supervisors: Prof.dr. J. Vles / Prof.dr. L. Zimmermann; Co-Supervisor: Dr. A. Gavilanes.

Els Vanhoutte: Peripheral Neuropathy outcome measures; Standardisation (PeriNomS) study part 2: Getting consensus. Supervisors: Prof.dr. C. Faber / Prof.dr. P. van Doorn; Co-Supervisor: Dr. I. Merkies, Spaarne ziekenhuis Hoofddorp.

Mayienne Bakkers: Small fibers, big troubles; diagnosis and implications of small fiber neuropathy. Supervisors: Prof.dr. C. Faber / Prof.dr. M. de Baets; Co-Supervisor: Dr. I. Merkies, Spaarne ziekenhuis Hoofddorp.

Ingrid Kramer: Zooming into the micro-level of experience: An approach for understanding and treating psychopathology. Supervisor: Prof.dr. J. van Os; Co-Supervisors: Dr. M. Wichers, UMC Groningen / Dr. C. Simons.

Esther Bouman: Risks and Benefits of Regional Anesthesia in the Perioperative Setting. Supervisors: Prof.dr. M. van Kleef / Prof.dr. M. Marcus, HMC, Qatar / Prof.dr. E. Joosten; Co-Supervisor: Dr. H. Gramke.

Mark Janssen: Selective stimulation of the subthalamic nucleus in Parkinson's disease; dream or near future. Supervisors: Prof.dr. Y. Temel / Prof.dr. V. Visser-Vandewalle, Keulen / Prof.dr. A. Benazzouz, Bordeax, France.

Reina de Kinderen: Health Technology Assessment in Epilepsy; economic evaluations and preference studies. Supervisors: Prof.dr. S. Evers / Prof.dr. A. Aldenkamp; Co-Supervisor: Dr. H. Majoie / Dr. D. Postulart, GGZ O-Brabant.

Saskia Ebus: Interictal epileptiform activity as a marker for clinical outcome. Supervisors: Prof.dr. A. Aldenkamp / Prof.dr. J. Arends, TUE / Prof.dr. P. Boon, Universiteit Gent, België.

Inge Knuts: Experimental and clinical studies into determinants of panic severity. Supervisor: Prof.dr. I. Myin-Germeys; Co-Supervisor: Dr. K. Schruers; Influencing panic.

Nienke Tielemans: Proactive coping post stroke: The Restored4Stroke Self-Management study. Supervisors: Prof.dr. C. van Heugten / Prof.dr. J. Visser-Meily, UMC Utrecht; Co-Supervisor: Dr. V. Schepers, UMC Utrecht.

Tom van Zundert: Improvements Towards Safer Extraglottic Airway Devices. Supervisors: Prof.dr. A.E.M. Marcus / Prof.dr. W. Buhre / Prof.dr. J.R. Brimacombe, Queensland, Australia / Prof.dr. C.A. Hagberg.

Tijmen van Assen: Anterior Cutaneous Nerve Entrapment Syndrome Epidemiology and surgical management. Supervisors: Prof.dr. G.L. Beets / Prof.dr. M. van Kleef / Dr. R.M.H. Roumen / Dr. M.R.M. Scheltinga, MMC Veldhoven.

Rohit Shetty: Understanding the Clinical, Immunological and Genetic Molecular Mechanisms of Keratoconus. Supervisors: Prof.dr. R.M.M.A. Nuijts / Prof.dr. C.A.B. Webers. 
Christine van der Leeuw: Blood, bones and brains; peripheral biological endophenotypes and their structural cerebral correlates in psychotic disorder. Supervisor: Prof.dr. J. van Os; Co-supervisor: Dr. M. Marcelis.

Sanne Peeters: The Idle Mind Never Rests; functional brain connectivity across the psychosis continuum. Supervisor: Prof.dr. J. van Os; Co-supervisor: dr. M. Marcelis.

Nick van Goethem: $\alpha 7$ nicotinic acetylcholine receptors and memory processes: mechanistic and behavioral studies. Supervisor: Prof.dr. H.W.M. Steinbusch; Co- supervisor: Dr. J. Prickaerts.

Nicole Leibold: A Breath of fear; a translational approach into the mechanisms of panic. Supervisor: Prof.dr. H.W.M. Steinbusch; Co-supervisors: Dr. K.R.J. Schruers / Dr. D.L.A. van den Hove.

Renske Hamel: The course of mild cognitive impairment and the role of comorbidity. Supervisor: Prof. dr. F.R.J. Verhey; Co-supervisors: Dr. I.H.G.B. Ramakers / Dr. P.J. Visser.

Lucia Speth: Effects of botulinum toxin A injections and bimanual task-oriented therapy on hand functions and bimanual activities in unilateral Cerebral Palsy. Supervisors: Prof.dr. J. Vles; Prof.dr. R. Smeets; Co-supervisor: Dr. Y. Janssen-Potten, Adelante Hoensbroek.

Yuan Tian: The effects of Lutein on the inflammatory pathways in age-related macular degeneration (AMD). Supervisors: Prof.dr. C. Webers; Prof.dr. A. Kijlstra, WUR; Co- supervisor: Dr. M. Spreeuwenberg; Dr. H. Tange.

Peggy Spauwen: Cognition and Type 2 diabetes; the interplay of risk factors. Supervisors: Prof.dr. F. Verhey; Prof.dr. C. Stehouwer; Co-supervisor: Dr. M. van Boxtel

Marc Hilhorst: Crescentic glomerulonephritis in ANCA associated vasculitis. Supervisors: Prof.dr. J. Cohen-Tervaert; Co-supervisor: Dr. P. van Paassen

Martin Gevonden: The odd one out: exploring the nature of the association between minority status and psychosis. Supervisors: Prof.dr. J-P. Selten; Prof.dr. J. Booij, Uva; Prof.dr. I. Myin-Germeys

Bart Biallosterski: Structural and functional aspects of sensory-motor Interaction in the urinary bladder. Supervisors: Prof.dr. Ph. Van Kerrebroeck; Prof.dr. S. De Wachter, UvAntwerpen; Co-supervisors: Dr. G. van Koeveringe; Dr. M. Rahnama'i.

Alexandra König: The use of information and communication technologies (ICT) for the assessment of patients with Alzheimer's Disease and related disorders. Supervisors: prof.dr. F. Verhey; prof. dr. Ph. Robert, Nice, Fr; Co-supervisors: dr. P. Aalten; dr. R. David, Nice. Fr.

Michelene Chenault: Assessing Readiness for Hearing Rehabilitation. Supervisors: prof.dr. M.P.F. Berger; prof.dr. B. Kremer; Co-supervisor: dr. L.J.C. Anteunis.

Anand Vinekar: Retinopathy of Prematurity. Recent advances in tele-medicine screening, risk factors and spectral domain optical coherence tomography imaging. Supervisor: prof.dr. C.A.B. Webers; Co-supervisor: dr. N.J. Bauer

Fleur van Dooren: Diabetes and Depression: exploring the Interface between Pathophysiological and Psychological factors. Supervisors: prof.dr. F.R.J. Verhey; prof.dr. J.K.L. Denollet, UvT; prof.dr. F. Pouwer, UvT; Co-supervisor: dr. M.T. Schram.

Gabriëlla Pons van Dijk: Taekwondo and physical fitness components in middle-aged healthy volunteers; the Sekwondo study. Supervisors: prof.dr. J. Lodder; prof.dr. H. Kingma; Cosupervisor: dr. A.F. Lenssen.

Yara Pujol López: Development and psychoneuroimmunological mechanisms in depression. Supervisor: prof.dr. H.W.M. Steinbusch; Co-supervisors: Dr. G. Kenis; Dr. D. van den Hove; Dr. Aye Mu Myint, München.

Romina Gentier: UBB+1; an important switch in the onset of Alzheimer's disease. Supervisors: Prof. H. Steinbusch; Prof. D. Hopkins; Co-supervisor: Dr. F. van Leeuwen. 
Sanne Smeets: Insights into insight: studies on awareness of deficits after acquired brain injury. Supervisor: Prof. C. van Heugten; Prof. R. Ponds; Co-supervisor: Dr. I. Winkens

Kim Beerhorst: Bone disease in chronic epilepsy: fit for a fracture. Supervisor: Prof. A. Aldenkamp; Prof. R. van Oostenbrugge; Co-supervisor: Dr. P. Verschuure.

Alex Zwanenburg: Cerebral and cardiac signal monitoring in fetal sheep with hypoxic- ischemic encephalopathy. Supervisor: Prof. T. Delhaas; Prof. B. Kramer; Co-supervisors: Dr. T. Wolfs; Dr. P. Andriessen, MMC.

Ismail Sinan Guloksuz: Biological mechanisms of environmental stressors in psychiatry. Supervisor: Prof. J. van Os; Co-supervisors: Dr. B. Rutten; Dr. M. Drukker.

Seyed Ehsan Pishva MD: Environmental Epigenetics in mental health and illness. Supervisor: Prof.dr. J. van Os; Co-supervisors: Dr. B.P.F. Rutten; Dr. G. Kenis.

Ankie Hamaekers: Rescue ventilation using expiratory ventilation assistance; innovating while clutching at straws. Supervisors: Prof.dr. W.F. Buhre; Prof.dr. M. van Kleef.

Rens Evers: 22q11.2 deletion syndrome: intelligence, psychopathology and neurochemistry at adult age. Supervisors: Prof.dr. L.M.G. Curfs; Prof.dr. T. v. Amelsvoort.

Sarah-Anna Hescham: Novel insights towards memory restoration. Supervisor: Prof.dr. Y. Temel; Cosupervisor: Dr. A. Blokland; Dr. A. Jahanshahi.

João P. da Costa Alvares Viegas Nunes: Insulin receptor sensitization improves affective pathology in various mouse models. Supervisor: Prof.dr. H.W.M. Steinbusch; Co- supervisors: Dr. K-P. Lesch; Dr. T. Strekalova; Dr.B.H. Cline, Oxford.

Yanny Ying-Yee Cheng: Clinical Outcomes After Innovative Lamellar Corneal Transplantation Surgery. Supervisor: Prof.dr. R.M.M.A. Nuijts; Co-supervisor: Dr. J.S.A.G. Schouten.

\section{6}

Oliver Gerlach: Parkinson's disease, deterioration during hospitalization. Supervisor: Prof.dr. R. van Oostenbrugge; Co-supervisor: Dr. W. Weber.

Remo Arts: Intracochlear electrical stimulation to suppress tinnitus. Supervisor: Prof.dr. R.J. Stokroos; Co-supervisor: Dr. E.L.J. Georg.

Mitchel van Eeden: The $€$ - Restore4stroke study: Economic evaluation of stroke care in the Netherlands. Supervisors: Prof.dr.mr. S.M.A.A. Evers; Prof.dr. C.M. v. Heugten; Co-supervisor: dr. G.A.P. van Mastrigt.

Pim Klarenbeek: Blood pressure and cerebral small vessel disease. Supervisor: Prof.dr. R.J. van Oostenbrugge; Co-supervisor: Dr. J. Staals.

Ramona Hohnen: Peripheral pharmacological targets to modify bladder contractility. Supervisor: Prof. dr. Ph.E.V. van Kerrebroeck; Co-supervisors: Dr. G.A. van Koeveringe; Dr. M.A. Sahnama'i; Dr. C. Meriaux.

Ersoy Kocabicak: Deep brain stimulation of the subthalamic nucleus: Clinical and scientific aspects. Supervisors: Prof.dr. Y. Temel; Prof.dr. K. van Overbeeke; Co-supervisor: Dr. A. Jahanshahi.

Sven Akkerman: Temporal aspects of cyclic messenger signaling in object recognition memory; a pharmalogical approach. Supervisor: Prof.dr. H.W.M. Steinbusch; Co-supervisors: dr. J. Prickaerts; dr. A. Blokland.

Anja Moonen: Emotion and Cognition in Parkinson's disease; etiology and neurobiological mechanisms. Supervisor: Prof.dr. F.R.J. Verhey; Co-supervisor: dr. A.F.G. Leentjens.

Anna Schüth: Three-dimensional bladder tissue morphology. Supervisors: Prof.dr. G.A. van Koeveringe; Prof.dr. M. v. Zandvoort, Aachen; Prof.dr. Ph. V. Kerrebroeck. 
Elisabeth van der Ven: Ethnic minority position as risk indicator for autism- Spectrum and psychotic disorders. Supervisors: Prof.dr. J.P. Selten; Prof.dr. J. van Os.

Zuzana Kasanova: Environmental reactivity for better or worse; The impact of stress and reward on neurochemistry, affect and behavior across the psychosis continuum. Supervisor: Prof.dr. I. MyinGermeys, KU Leuven/UM; Co-supervisor: dr. D. Collip.

Danielle Lambrechts: Ketogenic diet therapies; treatment for children and adults with refractory epilepsy. Supervisors: Prof.dr. H.J.M. Majoie; Prof.dr. J.S.H. Vles; Prof.dr. A.P. Aldenkamp; Cosupervisor: dr. A.J.A. de Louw, Kempenhaghe, Heeze.

Frank van Bussel: Advanced MRI in diabetes; cerebral biomarkers of cognitive decrements. Supervisors: Prof.dr.ir. W.H. Backes; Prof.dr. P.A.M. Hofman; Co-supervisor: dr. J.F.A. Jansen.

Lisa Schönfeldt: Neurostimulation to treat brain injury? Supervisors: Prof.dr. Y. Temel; Prof.dr. S. Hendrikx, Hasselt; Co-supervisor: dr. A. Jahanshahi.

Rianne Geerlings: Transition in patients with childhood-onset epilepsy; a long way to adulthood. Supervisor: Prof.dr. A.P. Aldenkamp; Co-supervisors:dr. A.J.A. de Louw, dr. L.M.C. Gottmer, Kempenhaeghe.

Nele Claes: B cells as multifactorial players in multiple sclerosis pathogenesis: insights from therapeutics. Supervisors: Prof.dr. V. Somers, Hasselt; Prof.dr. R. Hupperts Co-supervisors: Prof. dr. P. Stinissen, dr. J. Fraussen, Hasselt.

Olaf Schijns: Epilepsy surgery and biomarkers from history to molecular imaging. Supervisors: Prof.dr. J.J. van Overbeeke; Prof.dr. H. Clustermann, Aachen; Co-supervisors: dr. G. Hoogland; dr. M.J.P. v. Kroonenburgh.

Lizzy Boots: Balanced and Prepared; development and evaluation of a supportive e-health intervention for caregivers of people with early-stage dementia. Supervisors: Prof.dr. F.R.J. Verhey; Prof.dr. G.I.J.M. Kempen; Co-supervisor: dr. M.E. de Vugt.

Wouter Donders: Towards patient-specific (cerebro-) vascular model applications. Supervisors: Prof. dr. T. Delhaas; Prof.dr.ir. F.N. van de Vosse, TUE; Co-supervisor: dr.ir. W. Huberts.

Sizzle Vanterpool: The implications of intrauterine invasion by microbes for placental Pathology and the occurrence of adverse pregnancy outcomes. Supervisor: Prof.dr. B.W. Kramer. Co-supervisors: dr. J.V. Been, Erasmus MC Rotterdam, dr. U von Rango.

Manuela Heins: The Relationship between Social Adversity, Psychosis, and Depression across an Individual's Life Span. Supervisor: Prof.dr. I. Myin-Germeys.

Christianus van Ganzewinkel: NEONATAL PAIN; Out of Sight, Out of Mind? Supervisor: Prof.dr. B.W.W. Kramer; Co-supervisor: dr. P. Andriessen, MMC Veldhoven.

Anne-Hilde Muris: Hype or hope? Vitamin D in multiple sclerosis; A clinical and immunological perspective. Supervisor: Prof.dr. R.M.M. Hupperts; Co-supervisor: dr. J.G.M.C. Damoiseaux.

Gerard Bode: The link between ceramide transporters, innate Immunity and Alzheimer's disease. Supervisor: Prof.dr. M.H.V. de Baets; Co-supervisors: dr. P. Martinez, dr. M. Losen.

Jo Stevens: Advanced diagnostics and therapeutics for Alzheimer's disease. Supervisor: Prof.dr. M. de Baets; Co-supervisors: dr. M. Losen, dr. P. Martinez-Martinez.

Rosan Luijcks: Stress and pain in muscles and brain; developing psychophysiological paradigms to examine stress and pain interactions. Supervisors: Prof.dr. J.J. van Os; Prof.dr.ir. H.J. Hermens, UT; Co-supervisor: dr. R. Lousberg.

M.C. Haanschoten: Towards efficient cardiac surgery - the integrating role of anesthesiology and intensive care. Supervisors: Prof. dr. W. Buhre; Prof. dr. A. van Zundert (Queensland); Cosupervisors: Dr. M.A. Soliman Hamad; Dr. A. van Straten (Catharina zkhs.) 
Harmen Jan van de Haar: Microvascular and blood-brain barrier dysfunction in Alzheimer's disease. Supervisor: Prof.dr.ir. W. Backes; Prof.dr. F. Verhey; Co-supervisor: Dr. J. Jansen; Dr.ir. M. v. Osch, LUMC.

Coenraad Itz: Chronic low back pain, considerations about: Natural Course, Diagnosis, Interventional Treatment and Costs. Supervisor: Prof.dr. M. van Kleef; Prof.dr. F. Huygen, EUR; Co- supervisor: Dr. B. Ramaekers.

Willemijn Jansen: The Path of Alzheimer's disease: from neuropathology to clinic. Supervisor: Prof.dr. F. Verhey; Co-supervisors: Dr. P.J. Visser; Dr. I. Ramakers.

Ligia dos Santos Mendes Lemes Soares: Phosphodiesterase inhibitors: a potential therapeutic approach for ischemic cerebral injury. Supervisor: Prof.dr. H.W.M. Steinbusch; Co-supervisors: Dr. R.M. Weffort de Oliveira, Brazil; Dr. J. Prickaerts

Martijn Broen: Anxiety and depression in Parkinson's disease. Supervisor: Prof.dr. R.J. van Oostenbrugge; Co-supervisors: Dr. A.F.G. Leentjens; Dr. M.L. Kuijf.

Sandra Schipper: Extrasynaptic receptors as a treatment target in epilepsy. Supervisor: Prof.dr. J.H.S. Vles; Co-supervisors: Dr. G. Hoogland; Dr. S. Klinkenberg; Dr. M.W. Aalbers, RUG.

João Casaca Carreira: Making sense of Antisense Oligonucleotides Therapy in Experimental Huntington's disease. Supervisor: Prof.dr. Y. Temel; Co-supervisors: Dr. A. Jahanshahi; Dr. W. van Roon-Mom, LUMC.

Dominique IJff: Trick or Treat? Cognitive side-effects of antiepileptic treatment. Supervisors: Prof.dr. A.P. Aldenkamp; Prof.dr. M. Majoie; Co-supervisors: Dr. J. Jansen; Dr. R. Lazeron, Kempenhaeghe. Alfredo Ramirez: Neurogenetic approach in neurodegenerative disorders. Supervisors: Prof.dr. B.P.F. Rutten; Prof.dr. H.W.M. Steinbusch; Prof.dr. M.M. Nöthen, University of Bonn.

Nienke Visser: Toric Intraocular lenses in cataract surgery. Supervisor: Prof.dr. R.M.M.A. Nuijts; Cosupervisor: Dr. N.J.C. Bauer.

Jakob Burgstaller: Prognostic indicators for patients with degenerative lumbar spinal stenosis. Supervisor: Prof.dr. M. van Kleef; Co-supervisors: Dr. M.M. Wertli, University of Zurich; Dr. H.F. Gramke.

Mark van den Hurk: Neuronal Identity and Maturation: Insights from the Single-Cell Transcriptome. Supervisors: Prof.dr. H.W.M. Steinbusch; Prof.dr. B.P.F. Rutten; Co- supervisors: Dr. G. Kenis; Dr. C. Bardy, Adelaide.

Maria Nikiforou: Prenatal stress and the fetal gut. Potential interventions to prevent adverse outcomes. Supervisors: Prof.dr. B.W. Kramer; Prof.dr. H.W. Steinbusch; Co- supervisor: Dr. T.G. Wolfs.

Janneke Peijnenborgh: Assessment of cognition, time perception, and motivation in children. Supervisors: Prof.dr. J.S.H. Vles; Prof.dr. A.P. Aldenkamp; Co-supervisors: Dr. J. Hendriksen; Dr. P. Hurks.

Joany Millenaar: Young onset dementia; towards a better understanding of care needs and experiences. Supervisors: Prof.dr. F. Verhey; Prof.dr. R. Koopmans, RUN; Co- supervisors: Dr. M. de Vugt; Dr. C. Bakker, RUN.

\section{7}

Adriana Smits: Perinatal factors and hearing outcome. Supervisors: Prof.dr. R.J. Stokroos; Prof.dr. B.W. Kramer; Prof.dr. B. Kremer.

Angela Bouwmans: Transcranial sonography in parkinsonian disorders: clear window or blurred vision. Supervisor: Prof.dr. W.H. Mess; Co-promotores: Dr. W.E.J. Weber; Dr. A.F.G. Leentjens. 
Björn K. Stessel: Patient centred care after day surgery: scope for improvement. Supervisors: Prof.dr. W. Buhre; Prof.dr. B. Joosten. Co-supervisor: Dr. A.H. Gramke.

Jan Guy Bogaarts: Quantitative EEG and machine learning methods for the detection of epileptic seizures and cerebral asymmetry. Supervisor: Prof.dr. W.M. Mess; Co-supervisor: Dr.ir. J.P.H. Reulen; Dr.ir. E.D. Gommer.

Martin M. Müller: Pregnancy derived products for treatment of perinatal brain injuries. Supervisors: Prof.dr. B.W.W. Kramer; Prof.dr. D. Surbek, Bern; Co-supervisors: Dr. T. Wolfs; Dr. G. Gavilanes.

Daan Ophelders: Novel treatment strategies for the protection of the preterm brain; Re- balancing inflammation and regeneration. Supervisor: Prof.dr. B. Kramer; Co-supervisor: Dr. T. Wolfs; Dr. R. Jellema.

Rosalie van Knippenberg: Experience sampling in dementia care; an innovative intervention to support caregivers in daily life. Supervisors: Prof.dr. F. Verhey; Prof.dr. R. Ponds; Prof.dr. I. MyinGermeys, KU Leuven; Co-supervisor: Dr. M. de Vugt.

Claudia Vingerhoets: Investigating neurobiological mechanisms underlying comorbid cognitive symptoms in psychosis and substance use. Supervisors: Prof.dr. T. van Amelsvoort; Prof.dr. J. Booij, UvA; Co-supervisor: Dr. O. Bloemen

Dennis Oerlemans: Evolution of Neuromodulation for Lower Urinary Tract Dysfunction; Past, Present and Future. Supervisors: Prof.dr. Ph. van Kerrebroeck; Prof.dr. G. van Koeveringe. Co-supervisors: Dr. E. Weil; Dr. T. Marcelissen.

Marion Levy: Evaluation of BDNF/TrkB signaling as a common target in the treatment of major depression and Alzheimer's disease. Supervisors: Prof.dr. H. Steinbusch; Prof. L. Lanfumey, Université Paris Descartes, France. Co-supervisors: Dr. G. Kenis; Dr. D. van den Hove.

Patrick Domen: Stay connected: a family-based diffusion imaging study in psychotic disorder. Supervisor: Prof.dr. J. van Os. Co-supervisor: Dr. M. Marcelis

Geor Bakker: Innovative Approaches to Understanding the Neurobiology of Psychosis. Supervisors: Prof.dr. T. van Amelsfoort; Prof.dr. J. Booij, UvA. Co-supervisor: dr. M. Caan, UvA; dr. O. Bloemen.

Wilma Boevink: HEE! Over Herstel, Empowerment en Ervaringsdeskundigheid in de psychiatrie. Supervisors: Prof.dr. J. van Os; Prof.dr. Ph. Delespaul. Co-supervisor: dr. H. Kroon.

Nataliia Markova: Modified swim test as a mouse depression paradigm of enhanced cognitive processing: the role of GSK3ß. Supervisor: Prof.dr. H. Steinbusch; Prof.dr. K-P. Lesch, University of Wuerzburg. Co-supervisor: Dr. T. Strekalova.

Merijn van de Laar: Individual differences in insomnia; implications of psychological factors for diagnosis and treatment. Supervisor: Prof.dr. A. Aldenkamp; Prof.dr. D. Pevernagie, Universiteit Gent. Co-supervisor: Dr. S. Overeem, TUE.

Willem Buskermolen: If only I could tell ...; Measuring predictors for challenging behaviour in people with both intellectual disability and hearing impairment. Supervisor: Prof.dr. A. Aldenkamp. Cosupervisor: Dr. J. Hoekman, UL.

Kay Deckers: The role of lifestyle factors in primary prevention of dementia; an epidemiological perspective. Supervisor: Prof.dr. F. Verhey. Co-supervisor: Dr. M. van Boxtel; Dr. S. Köhler.

Brechje Dandachi-FitzGerald: Symptom validity in clinical assessments. Supervisors: Prof.dr. R. Ponds; Prof.dr. F. Verhey.

Maurice Theunissen: Understanding factors affecting postoperative Quality of Life. Supervisors: Prof. dr. M. Peters, Prof.dr. M. Marcus. Co-supervisor: Dr. H. Gramke.

Anna Cleutjens: COgnitive-Pulmonary Disease? Neuropsychological functioning in patients with COPD. Supervisors: Prof.dr. E. Wouters, Prof.dr. R. Ponds. Co-supervisors: Dr. D. Janssen, Horn, Dr. J. Dijkstra. 
Laura Serpero: Next Generaton Biomarkers in Perinatal Medicine: S100B Protein. Supervisors: Prof.dr. D. Gazzalo, Alessandria, Italy; Prof.dr. B..W.W. Kramer. Co-supervisor: Dr. A.W.D. Gavilanes.

Alessandro Varrica: S100B Protein and Congential Heart Diseases: Brain Aspects. Supervisors: Prof. dr. D. Gazzalo, Alessandria, Italy; Prof.dr. J.S.H. Vles; Prof.dr. L.J.I. Zimmermann. Co-supervisor: Dr. A.W.D. Gavilanes.

Pim R.A. Heckman: Targeting phosphodiesterase type 4 for improving cognitive fronto- striatal function: a translational approach. Supervisor: Prof.dr. J.G. Ramaekers. Co- supervisors: Dr. J.H.H.J.. Prickaerts; Dr. A. Blokland.

Sven van Poucke: Platelets, form sample to big data; exploring granularity in platelet research. Supervisors: Prof.dr. M.A.E. Marcus; Prof.dr. W. Buhre. Co-supervisor: Dr. M. Lancé.

Désirée M.J. Vrijens: Dysfunctions of the Lower Urinary Tract and Affective Symptoms. Supervisors: Prof.dr. Ph.E.V. van Kerrebroeck; Prof.dr. G.A. van Koeveringe. Co- supervisors: Dr. C. Leue.

Tamar van Veenendaal: Neurotransmitters \& Networks. An MR view on epilepsy and antiepileptic drugs. Supervisors: Prof.dr.ir. W.H. Backes; Prof.dr. A.P. Aldenkamp. Co- supervisor: Dr. J.F.A. Jansen.

Evelien M. Barendse: Autism Spectrum Disorders in High functioning Adolescents; Diagnostic considerations (AHA). Supervisors: Prof.dr. A.P. Aldenkamp; Prof.dr. R.P.C. Kessels, Radboud University.

Roy Lardenoije: A venture into the epigenetics of aging and Alzheimer's Disease. Supervisors: Prof.dr. B.P.F. Rutten; Prof.dr. H.W.M. Steinbusch. Co-supervisors: Dr. D. van den Hove; Dr. C.A. Lemere, USA.

Charlotte L. Mentzel: The course recognition and treatment of movement disorders in severe mental illness. Supervisors: Prof.dr. P.N. van Harten; Prof.dr. M.A.J. de Koning- Tijssen, UMCG. Cosupervisor: Dr. P.R. Bakker.

Tim Batink: Third Wave Behaviour Therapy: Process Measures and Contextual Interventions. Supervisors: Prof.dr. F.P.M.L. Peeters; Prof.dr. J.J. van Os; Prof.dr. M.C. Wichers, UMC Groningen.

Kevin L.J. Rademakers: Detrusor Underactivity: From Theory To Clinical Assessment. Supervisors: Prof. dr. G.A. van Koeveringe; Prof.dr. Ph.E.V. van Kerrebroeck. Co-supervisor: Dr. M. Oelke. 
Addendum 


\section{LIST OF PUBLICATIONS}

\section{INTERNATIONAL JOURNALS}

Douven E, Köhler S, Schievink SHJ, van Oostenbrugge RJ, Staals J, Verhey FRJ, Aalten P. Temporal associations between fatigue, depression, and apathy after stroke: results of the CASPER study. Cerebrovascular Diseases 2017; 44:330-337; doi:10.1159/000481577.

Douven E, Köhler S, Schievink SHJ, van Oostenbrugge RJ, Staals J, Verhey FRJ, Aalten P. Baseline vascular cognitive impairment predicts the course of apathetic symptoms after stroke: the CASPER study. The American Journal of Geriatric Psychiatry 2017; doi:10.1016/j.jagp.2017.09.022.

Douven E, Köhler S, Rodriguez MMF, Staals J, Verhey FRJ, Aalten P. Imaging Markers of Post-Stroke Depression and Apathy: a Systematic Review and Meta-Analysis. Neuropsychology Review 2017;27(3):202-219.

Douven E, Schievink SHJ, Verhey FRJ, van Oostenbrugge RJ, Aalten P, Staals J, Köhler S. The Cognition and Affect after Stroke-a Prospective Evaluation of Risks (CASPER) study: rationale and design. BMC Neurology 2016;16(1):1-11.

\section{NATIONAL JOURNAL}

Schievink, S., Douven, E., Aalten, P., \& Köhler, S. (2014). Neuropsychiatrische syndromen na eenberoerte. Neuropraxis, 18(5):151-161.

\section{SUBMITTED}

Douven E, Staals J, Schievink SHJ, van Oostenbrugge RJ, Verhey FRJ, WetzelsMeertens S, Köhler S, Aalten P. Premorbid personality and course of symptoms of depression and apathy after stroke: results of the CASPER study.

Douven E, Staals J, Schievink SHJ, Freeze WM, Hellebrekers DMJ, Wolz R, van Oostenbrugge RJ, Verhey FRJ, Köhler S, Aalten P. Imaging markers associated with the development of post-stroke depression and apathy: results of the CASPER study.

Douven E, Aalten P, Staals J, Schievink SHJ, van Oostenbrugge RJ, Verhey FRJ, Köhler S. Depression-Executive Syndrome after stroke: results of the CASPER study.

Schievink SHJ, Douven E, Aalten P, Staals J, Freeze WM, Hellebrekers DMJ, Wolz $\mathrm{R}$, van Oostenbrugge RJ, Verhey FRJ, Köhler S. The role of volumetric MRI markers of white matter hyperintensities, hippocampal volume, and global atrophy in post-stroke cognitive functioning. 
Addendum 


\section{CURRICULUM VITAE}

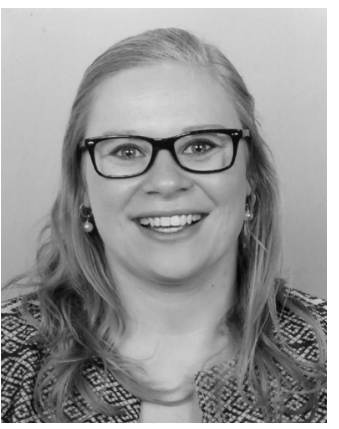

Elles Douven was born on February 191991 in NederweertEind, the Netherlands. After graduating from high school (Philips van Horne, Weert) in 2009, she started to study Psychology at Maastricht University and graduated cum laude in 2012. During her bachelor she also completed the Honours program of the Faculty of Psychology and Neuroscience. In the same year, Elles started the two-year Research Master Neuropsychology at Maastricht University. She performed a research internship on the CASPER study at the department of Psychiatry and Neuropsychology of Maastricht University. She also performed a clinical internship at the Memory Clinic of Maastricht University Medical Center in Maastricht and acquired her psychodiagnostics registration (BAPD). In 2014 she graduated and started working as a PhD candidate on the CASPER study at the Department of Psychiatry and Neuropsychology of Maastricht University under supervision of Prof. dr. Frans Verhey, Dr. Pauline Aalten, and Dr. Julie Staals. Currently, Elles is working as a postdoctoral researcher at the department of Psychiatry and Neuropsychology at Maastricht University and Alzheimer Center Limburg.

Elles Douven werd geboren op 19 februari 1991 te Nederweert-Eind. In 2009 behaalde ze haar VWO diploma op de Philips van Horne te Weert. Hierna begon zij haar studie Psychologie aan de Universiteit van Maastricht. In 2012 werd haar bachelor diploma cum laude behaald. Tijdens haar bachelor rondde zij ook het Honours programma af van de Faculteit Psychologie en Neurowetenschappen. In hetzelfde jaar begon Elles met de tweejarige onderzoeksmaster Neuropsychologie aan de Universiteit van Maastricht. Als onderdeel van deze master heeft zij een onderzoeksstage gedaan bij de afdeling Psychiatrie en Neuropsychologie van de Universiteit Maastricht op de CASPER studie. Ze liep ook een klinische stage op de geheugenpoli in het Maastricht Universitair Medisch Centrum waarvoor zij haar psychodiagnostiek registratie behaalde (BAPD). In 2014 studeerde ze af en begon als promovenda op de CASPER studie bij de afdeling Psychiatrie en Neuropsychologie van de Universiteit Maastricht onder supervisie van Prof. dr. Frans Verhey, Dr. Pauline Aalten en Dr. Julie Staals. Momenteel is Elles werkzaam als postdoc onderzoeker op de afdeling Psychiatrie en Neuropsychologie van Maastricht University en Alzheimer Centrum Limburg. 
